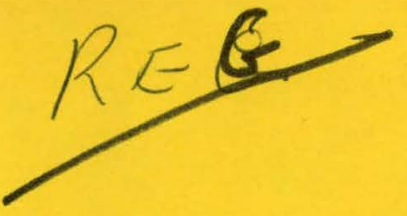

\title{
A Biblography for the Satellite Power System (SPS) Concept Development and Evaluation Program
}

April 1981<smiles>[2H][Mg]</smiles>

Prepared for:

U.S. Department of Energy

Office of Energy Research

Solar Power Satellite Project Division

Under Contract Nô/31-109-ENG-38

DOE/NASA

Satellite Power System

Concept Development

and

Evaluation Program 


\section{DISCLAIMER}

This report was prepared as an account of work sponsored by an agency of the United States Government. Neither the United States Government nor any agency Thereof, nor any of their employees, makes any warranty, express or implied, or assumes any legal liability or responsibility for the accuracy, completeness, or usefulness of any information, apparatus, product, or process disclosed, or represents that its use would not infringe privately owned rights. Reference herein to any specific commercial product, process, or service by trade name, trademark, manufacturer, or otherwise does not necessarily constitute or imply its endorsement, recommendation, or favoring by the United States Government or any agency thereof. The views and opinions of authors expressed herein do not necessarily state or reflect those of the United States Government or any agency thereof. 


\section{DISCLAIMER}

Portions of this document may be illegible in electronic image products. Images are produced from the best available original document. 


\section{NOTICE}

This report was prepared as an account of work sponsored by the United States Government. Neither the United States nor the United States Department of Energy, nor any of their employees, makes any warranty, express or implied, or assumes any legal liability or responsibility for the accuracy, completeness, or usefulness of any information, apparatus, product, or process disclosed, or represents that its use would not infringe privately owned rights. Reference herein to any specific commercial product, process, or service by trade name, mark, manufacturer, or otherwise, does not necessarily constitute or imply its endorsement, recommendation, or favoring by the United States Government or any agency thereof. The views and opinions of authors expressed herein do not necessarily state or reflect those of the United States Government or any agency thereof.

Avallable from:

National Technical Information Service (NTIS)

U.S. Department of Commerce

5285 Port Royal Road

Springfield, Virginia 22161

$\begin{array}{ll}\text { Price: } & \begin{array}{l}\text { Printed Copy: } \\ \text { Microfiche: }\end{array} \\ & \$ 21.50 \\ & \$ 4.00\end{array}$ 


\section{A Biblography for the Satellite Power System (SPS) Concept Development and Evaluation Program}

April 1981

Prepared by:

M. Abromavage, R. Calzadilla and Mark Murray

Argonne National Laboratory

Argonne, IL 60439

Under Contract No.^31-109-ENG-38

Prepared for:

\section{U.S. Department of Energy}

Office of Energy Research

Solar Power Satellite Project Division

Washington,D.C. 20585

\section{DOE/NASA}

Satellite Power System

Concept Development and

Evaluation Program

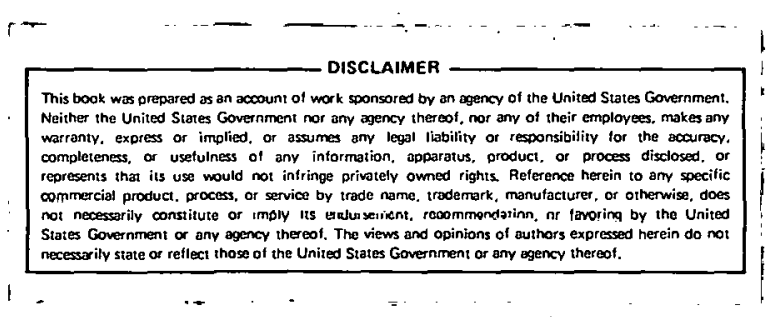

DISTRBBUTIOH OF THIS DOCUMENT IS UHLLMITED 


\section{FOREWORD}

This bibliography is the final publication in the Department of Energy and the National Aeronautics and Space Administration series on the Satellite Power System Concept Development and Evaluation Program. The Satellite Power System Concept was initially developed by Peter Glaser in 1968. Commencing in 1977 and continuing through 1980, a comprehensive assessment of this concept was conducted using a Reference System as a "straw man", and encompassed systems definition and engineering aspects; environmental assessment of microwave health and ecology, risks to space workers and atmospheric effects; a societal assessment covering resource requirements (land and materials), international and institutional issues; and a comparative assessment of the SPS Reference System relative to other advanced energy technologies such as fusion and improved current energy technologies.

The National Aeronautics and Space Administration and its contractors investigated various system design concepts and initiated some prel iminary work in critical technological and engineering areas. The Department of Energy, assisted by several national laboratories, other government organizations such as the Environmental Protection Agency and the Institute for Telecommunications Sciences, and independent contractors performed the environmental, societal, and comparative assessments.

This bibliography, prepared by Martin Abromavage, Rosa Calzadilla and Mark Murray, includes the many reports written during the three-year SPS Concept Development and Evaluation Program. A large quantity of professional papers were also written by both contributing and independent irivestigators in the United States and abroad.

It is hoped that this bibliography will be useful to those who are interested in the concept of collecting energy in space and using it on Earth.

F. A. Koomanoff, Director.

Solar Power Satellite Projects Division Office of Energy Research 
THIS PAGE

\section{WAS INTENTIONALLY LEFT BLANK}




\section{CONTENTS}

FORÉWORD ........................

CONTENTS ................................

INTRODUCTION AND USERS' GUIDE ............. v

Subject Categories ............... vi

Relationships Between Subject Categories....... viii

Key Words . . . . . . . . . . . . . . ix

Acquisition Numbers and Sources ......... ix

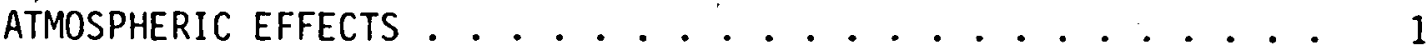

BIOLOGIC EFFECTS ..................... 21

COMPARATIVE ASSESSMENT ...................... 51

ENERGY ALTERNATIVES AND POLICY . . . . . . . . . . . . . . . . .

ELECTROMAGNETIC COMPATIBILITY . . . . . . . . . 79

ENVIRONMENTAL EFFECTS ................. 87

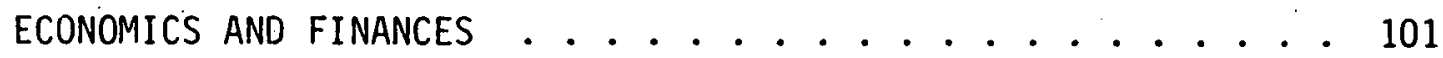

PROGRAM PLANS ..................... 109

POWER GENERATION AND TRANSMISSION . . . . . . . . . . . 119

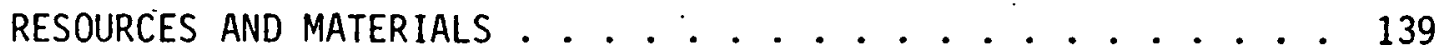

SYSTEMS ANALYSES . . . . . . . . . . . . 147

SYSTEMS ENGINEERING . . . . . . . . . . . 173

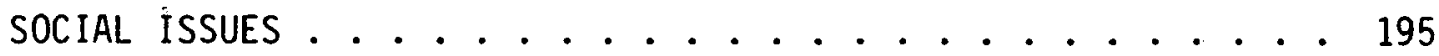

SPACE TRANSPORTATION . . . . . . . . . . 215

SPACE UTILIZATION . . . . . . . . . . . . 223

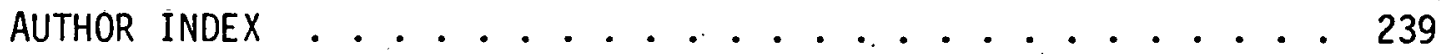

ACQUISITION SOURCES ................ 247

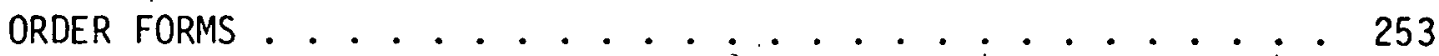


THIS PAGE

\section{WAS INTENTIONALLY LEFT BLANK}


The Satellite Power System Concept Development and Evaluation Program (SPS-CDEP) jointly conducted by the U. S. Department of Energy (DOE) and the National Aeronautics and Space Administration (NASA) was completed in late 1980. A large number of reports were prepared under the auspices of the SPS Project Division, Office of Energy Research, during the three-year program. Many domestic and foreign organizations (industries, governments, and professional and trade groups) also studied the SPS energy concept independently and prepared reports and papers on the subject. Literally hundreds of professional papers on SPS have appeared in engineering and scientific journals, and popular magazines and newspapers have reported extensively on SPS.

The results of the three-year CDEP have been reported by the Department of Energy and NASA. Reports also are being prepared independently by the Congress' Office of Technology Assessment (OTA) and the National Research Council (NRC) of the National Academy of Sciences, but are not yet complete.

A11 reports prepared under the auspices of DOE and NASA during the CDEP are traceable and available to the public, and are listed here. Some especial$7 y$ important reports prepared by NASA and its contractors prior to 1978 also are included. Citation cards also are included for the OTA and NRC reports expected to be completed by mid - 1981, as is a card for the final comparative assessment report prepared for SPS-CDEP which is expected to be avaialble in the early spring of 1981 .

A sampling of professional papers is included to illustrate the wide interest in SPS among scientists and engineers. Those which are cited should not be regarded as more noteworthy than others which may have been prepared but not included here.

Two rules were followed in preparing this bibliography. Each report or paper had to be on file in the SPS Project Division, and each had to be traceable to a source readily available to the public. Reports with restrictions on distribution and copyrighted papers not traceable to a publishing source were therefore excluded. 
Finally, a note about cited authors is appropriate. Many investigators who contributed significant work during SPS-CDEP may not be listed. There are several reasons for this. Government regulations concerning acknowledgements have changed from time to time, and in some cases individuals who contributed to reports through subcontracts with national laboratories or prime contractors could not be listed among principal authors. Some organizations issue reports as corporate documents without listing individual authors. Conference and workshop proceedings may include papers by dozens of contributors, but a citation to the entire document usually attributes the work to the editors of the proceedings. Thus the author index should not be regarded as an exclusive list of important contributors to SPS-CDEP.

Subject Categories

Reports and articles are categorized in fifteen subject areas. The areas were selected according to major interest in aspects of SPS illustrated during the CDEP. The CDEP, 1ike any assessment process, had to be wide-ranging and well-integrated in order to be sucessful. For example, investigators concerned with microwave power generation and transmission had to be aware of its environmental implications. Those concerned with societal assessment had to be aware of microwave exposure rules and regulations. Thus the issue of microwave biologic effects may be addressed in reports in all three categories, but with different emphasis on aspects of the issue.

CATEGORY

AE - Atmospheric Effects

BE - Biologic Effects

CA - Comparative Analysis

\section{EMPHASIS}

Effects which SPS activities might have on the atmosphere from near the earth's surface to geostationary earth orbit altitudes, including effects due to ionospheric heating.

Potential health and safety effects on humans, animals and plants which might be produced directly by SPS activities or indirectly by support activities (mining, manufacturing, etc.), Including effects due to ionizing radiation in space.

Information which is useful for comparing SPS performance, cost and residual effects with other selected current and emerging energy technologies. 
CATEGORY

EA - Energy Alternatives and Policy

EC - Electromagnetic Compatibility

EE - Environmental Effects

$E F$ - Economics and Finances

PP - Program Plans

PT - Power Generation and Transmission

RM - Resources and Materials

SA - Systems Analyses

\section{EMPHASIS}

Information which provides a useful perspective of SPS and several other energy technologies vis-a-vis national and international energy concerns.

Telecommunications interference effects and other systems performance degradations potentially caused by radio wave propagation disturbances or direct energy coupling with SPS microwave energy, except for interference due to ionospheric heating.

A wide range of potential environmental effects which might be produced by SPS. Documents generally cover generic environmental issues or more than one particular type of effect.

Documents which primarily discuss costs of fully developing an operational SPS and methods for financing operations. Does not include socio-economic effects which might accompany the development and operation of SPS.

Plans and planning concepts for SPS/CDEP and other selected energy alternatives.

Technical reports and papers which principally address engineering concepts, designs, theoretical and experimental analysis and the engineering economics of generating and transmitting useful energy from space to earth. Does not include terrestrial power transmission and generation.

Reports and papers identifying, inventorying and evaluating the need for and potential effects on resources and materials which may be required to fully develop SPS, including land.

Documents which present either brief overview analyses or descriptions of SPS, or provide somewhat detailed evaluations of SPS and its broad characteristics. Does not include engineering evaluations of specific SPS components, 
CATEGORY

SE - Systems Engineering

SI - Social Issues

ST - Space Transportation

SU - Space Utilization
EMPHASIS

Engineering reports on particular SPS components, characteristics, parameters and technology issues, including rectenna engineering but excluding space transportation.

Reports and articles concerning significant social issues, international concerns and interests, institutional concerns and issues and public concerns and interest in and about SPS.

Technical and scientific information on SPS space transportation requirements, concepts (including earth-to-spare and return), operating scenarios and econom$i c s$, and similar information on other. space ventures germane to SPS.

Principally a collection of papers from the technical and scientific space community on the potential, benefits and adverse effects which might accompany society's exploitation of space.

Relationships between Subject Categories

The nature of conceptual programs and evaluation processes makes it virtually impossible to classify documents according to distinct categories. Program work must be integrated and coordinated among participating groups in urder to be effective, and it follows that many: reports resulting from the work could be classified in a number of different ways. The judgments used for classifying documents included in this bibliography were based mainly on the principal content or intent of a particular document, but some arbitrary classification was occasionally necessary. The chief criterion in these cases was to classify the document in the most definitive category. For example, a document which might include substantial information on microwave biologic effects and was somewhat less definitive on other environmental issues was classified in the Biologic Effects category rather than in the broader Environmental Effects category.

The foregoing suggests the user should not concentrate on only one particular category of documents in using this bibliography. While it would be worthwhile to concentrate initially on a particular category of interest, a 
review of related subject categories is encouraged to ensure a comprehensive understanding of SPS/CDEP. Related categories are listed in each section of this bibliography for convenience.

Key Words

Key words are listed for each citation. A maximum of six key words is provided for each document. A list of key words is included for each subject category and page numbers are provided for each citation for which the key word applies in each category. A universal system of key words is not used. They were selected to portray specific SPS/CDEP issues and interests, and should not be used to correlate with other bibliographies. It should be recognized that the practical limit of six key words should not lead the user to presume that no other information is included in a particular document. The six key words do highlight the most definitive information in a document, however.

Acquisition Number and Sources

Acquisition numbers are included for each citation. Muitiple acquisition numbers should be used (where listed) when requesting documents from identified sources. 


\section{ATMOSPHERIC EFFECTS}

(CATEGORY AE)

Documents in this subject category cover potential atmospheric effects which might be produced by SPS launch and space vehicle exhaust effluents, microwave power transmission and rectenna operations. The effects of the atmosphere on SPS parameters also are included in this category, as are documents which describe atmospheric characteristics and effects of other space activities on the atmosphere.

KEY WORDS:

Analysis Methods

Astronomy

Atmospheric Models

Biologic Effects

Climate Effects

Construction Effects

Ecology Impacts

Electric Charge

Exhaust Emissions

Experiments

Frequency Scaling

Geophysics

Heater Facilities

Heating Effects

Heating Theory

Interference Effects

Ionosphere Heating

Ionosphere Predictions

Laser Transmission

Launch Effects

Lightning

Magnetosphere Effects

Mesospheric Effects

Microwave Heating

Microwave Propagation

Models

Particulate Exhausts

Physical Processes

Pilot Signal

Plasma Effects.
PAGE NUMBER:

$5,6,10,13,14,15,16,17,18$

8

$3,5,7,10,16,18,19$

11

$3,4,5,6,11,12,14,15,16$

3

10

7,8

$3,5,6,7,8,13,14,15,16,17,18,19$

$4,6,9,11,12,13,14,18,19$

$9,14,17$

7,8

$4,9,14$

$3,10,17$

$9,10,17$

$4,9,10,11,13,14,17$

$4,9,10,11,14,17,18$

$3,4,5,9,10,11,15,17,18,19$

5

$6,10,13,14,16$

15

$4,7,8,12,15$

6

$3,4,8$

8,12

7,8

11,14

$3,4,5,7,12,13,15,16,18,19$

11

$4,5,7,9$ 
(Atmospheric Effects Category AE continued)

KEY WORDS

Plasmasphere "Effects

Power Losses

Propellant Characteristics

Rectenna Heating

Rectenna Protection

Reflected Light

Satellite Drag

Space Shuttle

Stratospheric Effects

Transportation Effects

Troposphere Effects

Weather Effects
PAGE NUMBER:

$$
\begin{aligned}
& 15,11,15 \\
& 5,11,14 \\
& 3,12 \\
& 15 \\
& 8 \\
& 8 \\
& 10,11 \\
& 6,11 \\
& 3,5,6,13,14,15,16,18,19 \\
& 10 \\
& 5,15
\end{aligned}
$$

RELATED CATEGORIES:

Electromagnetic Compatibility (EC)

Envirironmental Effects (EE)

Power Generation and Transmission (PT)

Systems Engineering (SE)

Space Transportation (ST)

2 


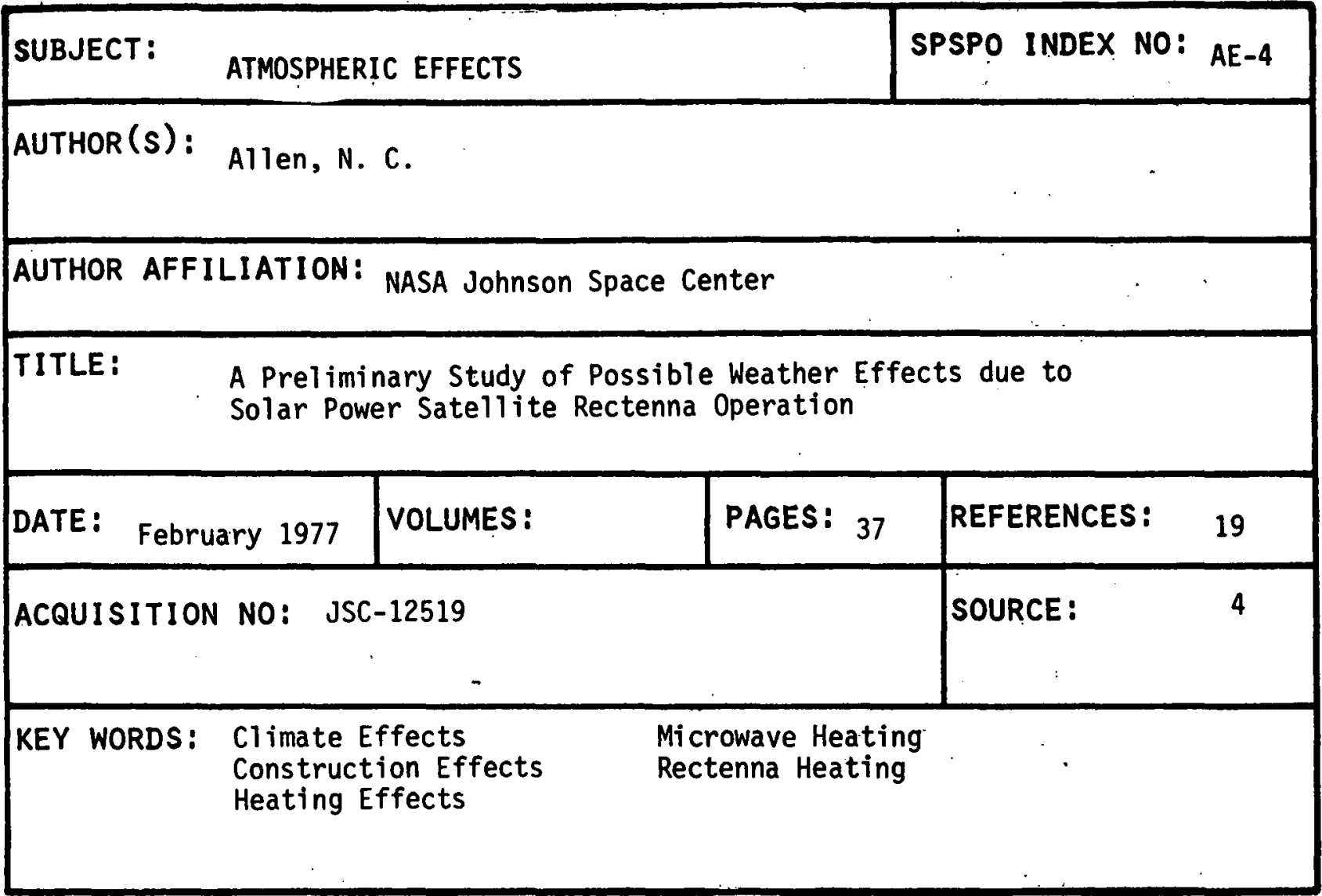

SUBJECT: ATMOSPHERIC EFFECTS

AUTHOR(S): Ames Research Center

AUTHOR AFFILIATION: National Aeronautics and Space Administration

TITLE: Environmental Impacts of the Satellite Power System (SPS) on the Middle Atmosphere

DATE: October 1980

VOLUMES:

PAGES: 36 REFERENCES: 68 .

ACQUISITION NO: DOE/ER/110035-01 SOURCE: 5

KEY WORDS: Atmospheric Models

Cl imate Effects

Exhaust Emissions

Ionosphere Predictions

Physical Processes

Transportation Effects 


$\begin{array}{lll}\text { AUTHOR (S): } & \text { (1) Basu, S. } & \text { (2) Klobuchar, J. A. } \\ & \text { (2) Johnson, A. L. } & \text { (3) Rush, C. M. }\end{array}$

$\begin{array}{ll}\text { AUTHOR AFFILIATION: (1) Emmanuel College } & \text { (3) U. S. Department of Commerce } \\ & \text { (2) U. S. Air Force }\end{array}$

TITLE: Preliminary Results of Scintillation Measurements Associated with Ionosphere Heating and Possible Implications for the Solar Power Satellite

\begin{tabular}{|l|l|l|l|}
\hline DATE: August 1980 & VOLUMES: & PAGES: 4. & REFERENCES: 25 \\
\hline ACQUISITION NO: 0094-8276/80/0080L-0R8ח & SOURCE: 36 \\
\hline KEY WORDS: $\begin{array}{l}\text { Experiments } \\
\text { Heater Facilities } \\
\text { Heating Effects }\end{array}$ & $\begin{array}{l}\text { Interference Effects } \\
\text { Ionosphere Heating } \\
\text { Microwave Heating }\end{array}$ \\
\hline
\end{tabular}

SUBJECT: ATMOSPHERIC EFFECTS

$\operatorname{AUTHOR}(s)$ : Bauer, E. (Ed.)

AUTHOR AFFILIATION: Lá Jolla Institute

TITLE: Proceedings of the Workshop on the Modification of the Upper Atmosphere by Satellite Power System (SPS) Propulsion Effluents

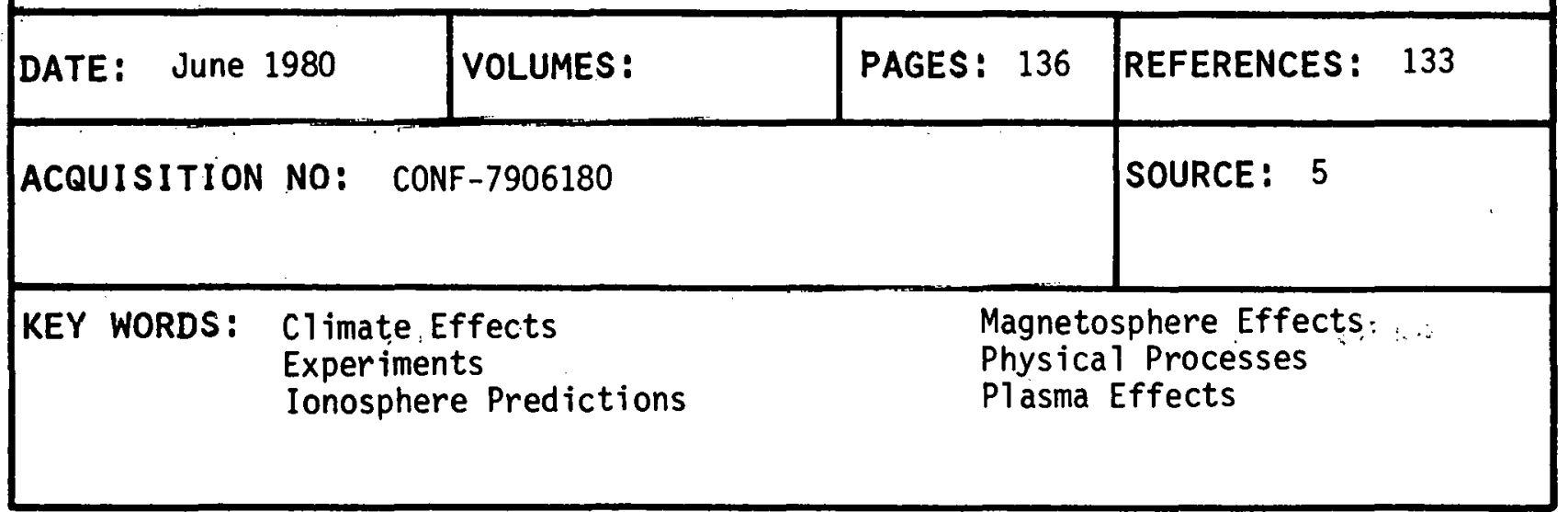


AUTHOR (s): Bernhardt, P.A.

Price, K. M.

da Rósa, A. V.

\section{AUTHOR AFFILIATION: Stanford University}

TITLE: Ionospheric Modification by Rocket Effluents

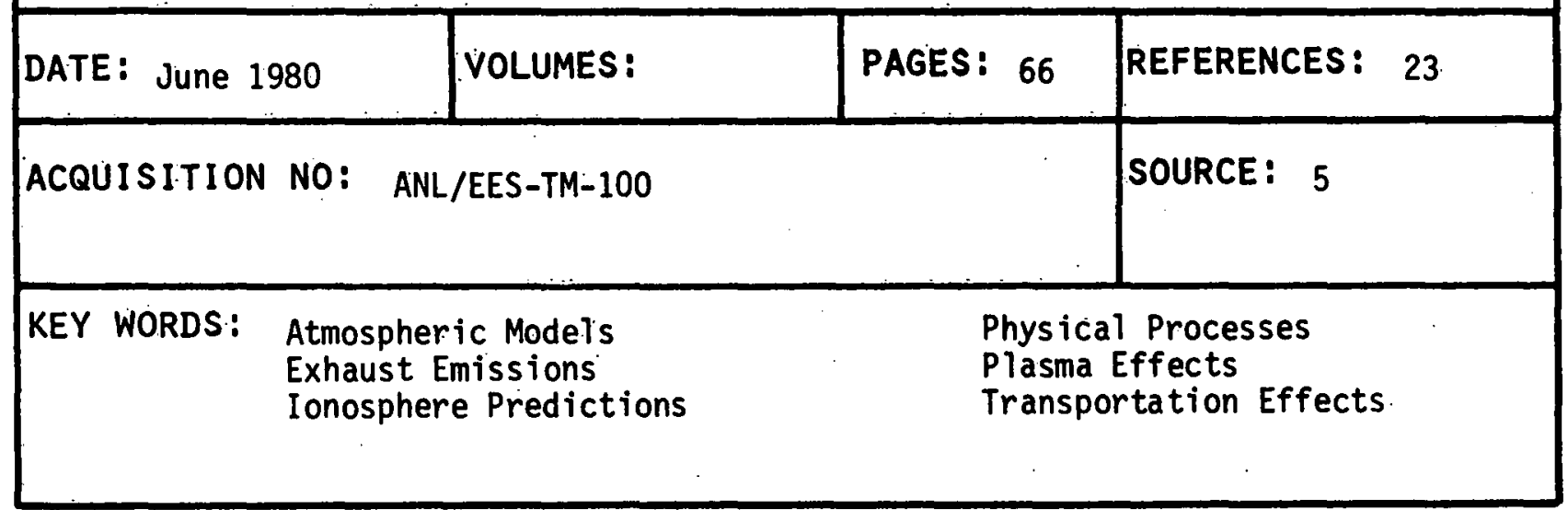

\section{SUBJECT: ATMOSPHERIC EFFECTS}

SPSPO INDEX NO: AE-29

$\operatorname{AUTHOR}(S)$ : Beverly, R. E.

AUTHOR AFFILIATION: Rockwell International

TITLE: Satellite Power Systems (SPS) Laser Studies; Volume II: Meteorological Effects on Laser Beam Propagation and Direct Solar Pumped Lasers for the SPS

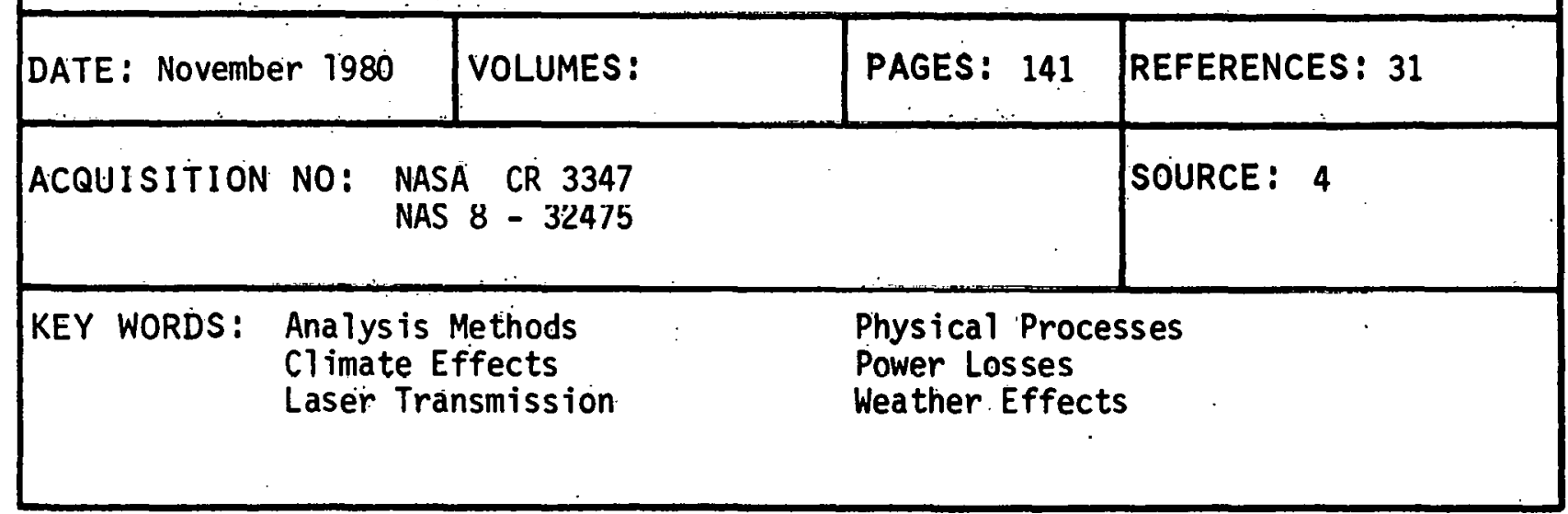


AUTHOR(S): Brubaker, K. L.

AUTHOR AFFILIATION: Argonne National Laboratory

TITLE: Proceedings of the Workshop on Stratospheric and Mesospheric Impacts of Satellite Power Systems (SPS)

\begin{tabular}{|l|l|l|l|}
\hline DATE: December 1979 & VOLUMES: & PAGES: 112 & REFERENCES: 43 \\
\hline ACQUISITION NO: CONF-7809197 & SOURCE: & 5 \\
\hline KEY WORDS: & $\begin{array}{l}\text { Climate Effects } \\
\text { Exhaust Emissions } \\
\text { Launch Effects }\end{array}$ & $\begin{array}{l}\text { Mesospheric Effects } \\
\text { Stratospheric Effects } \\
\text { Transportation Effects }\end{array}$ \\
\hline
\end{tabular}

SUBJECT: ATMOSPHERIC EFFECTS

SPSPO INDEX NO: AE-30

AUTHOR(s): Chackn, C. C..

Mendilio. M.

AUTHOR AFFILIATION: Boston University

TITLE: A Search of Archived Data Sources For Rocket Exhaust-Induced Modifications of the Ionosphere

\begin{tabular}{ll} 
DATE: September 1980 VOLUMES: \\
\hline
\end{tabular}

PAGES: $49 \cdot$ REFERENCES: 23

ACQUISITION NO: ANL/EES-TM-122

SOURCE : 5

KEY WORDS: Analys is Methods

Exhaust Emissions

Experiments 
AUTHOR(s): Chiu, Y. T.

Luhmann, J. G.

Schuiz, M.

AUTHOR AFFILIATION: The Aerospace Corporation

TITLE: Magnetospheric Effects of Ion and Atom Injections by the Satellite Power System

DATE: JuTy 1980 VOLUMES: \begin{tabular}{ll|l} 
PAGES : 118 REFERENCES: 61
\end{tabular}

ACQUISITION NO: ANL/EES-TM-94

SOURCE : 5

KEY WORDS: Atmospheric Models

Physical Processes

Exhaust Emissions

Plasma Effects

Magnetosphere Effects

Propellant Characteristics

\section{SUBJECT: ATMOSPHERIC EFFECTS}

AUTHOR(S): CrisweII, D. R.

De, B. R.

AUTHOR AFFILIATION: Lunar Science Institute

TITLE: Intense Localized Photoelectric Charging in the Lunar Sunset Terminator Region - 2. Supercharging at the Progression of Sunset

\begin{tabular}{|l|l|}
\hline DATE: March 1977 & VOLUMES: \\
\hline
\end{tabular}

ACQUISITION NO: 6A 0968 \begin{tabular}{l|l} 
PAGES: 3 & REFERENCES: 5 \\
\hline
\end{tabular}

$\begin{array}{cl}\text { KEY WORDS: } & \begin{array}{l}\text { Electric Charge } \\ \text { Geophysics } \\ \text { Models }\end{array}\end{array}$




\section{SUBJECT ATMOSPHERIC EFFECTS}

AUTHOR(S): De, B. R.

Criswel1, D. R.

\section{AUTHOR AFFILIATION: Lunar Science Institute}

TITLE: Intense Localized Photoelectric Charging in the Lunar Sunset

Terminator Region - 1. Development of Potentials and Fields

\begin{tabular}{|c|c|c|c|}
\hline DATE: March 197 & VOLUMES: & PAGES: 6 & REFERENCES : 15 \\
\hline \multicolumn{3}{|c|}{ ACQUISITION NO: $6 A 0967$} & SOURCE : 36 \\
\hline KEY WORDS: $\begin{array}{l}\text { EI } \\
\mathrm{Ge} \\
\mathrm{Mo}\end{array}$ & \multicolumn{2}{|c|}{$\begin{array}{l}\text { Electric Charge } \\
\text { Geophysics } \\
\text { Models }\end{array}$} & \\
\hline
\end{tabular}

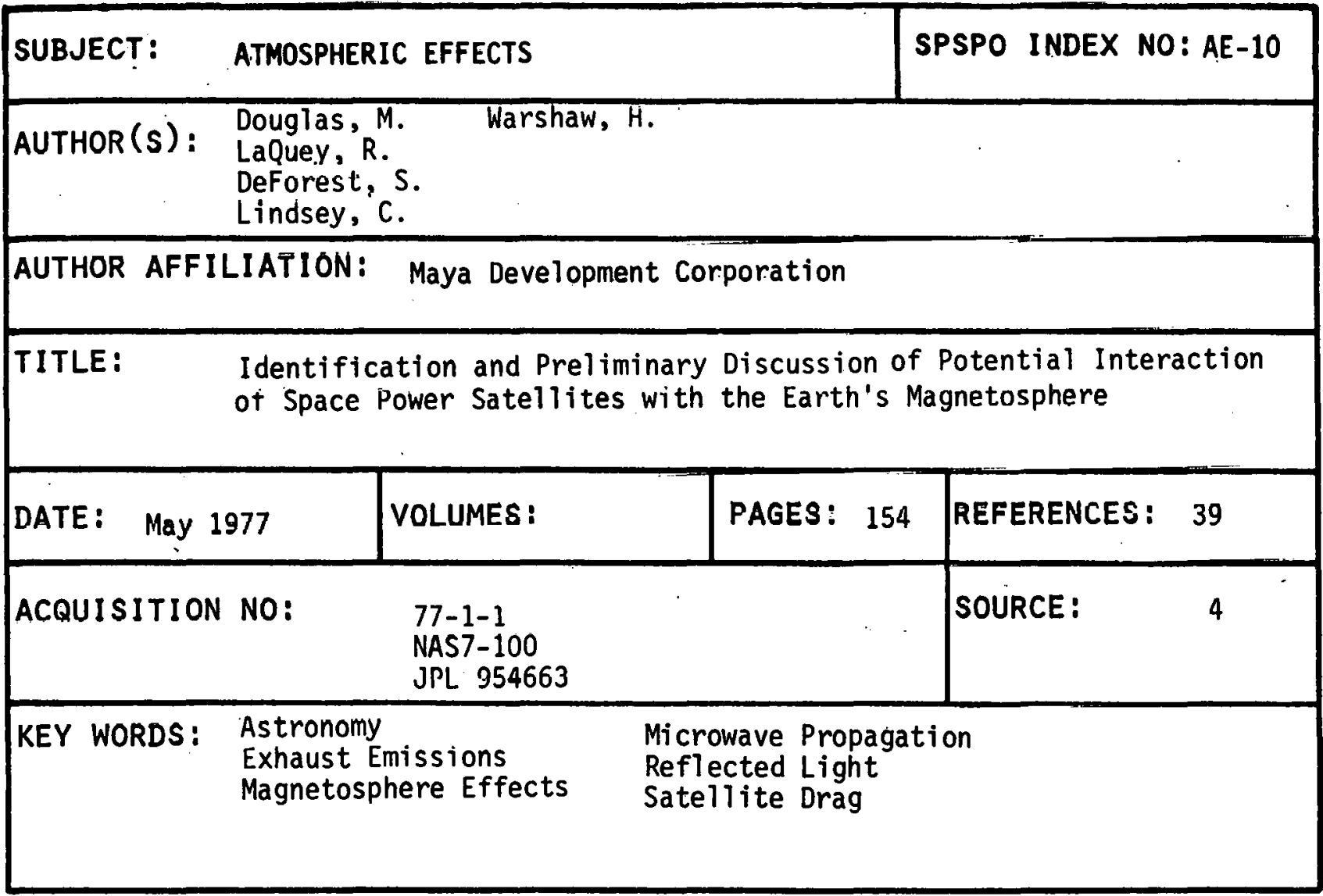




\begin{tabular}{|c|c|c|c|c|c|}
\hline SUBJECT: & \multicolumn{3}{|c|}{ ATMOSPHERIC EFFECTS } & \multicolumn{2}{|c|}{ SPSPO INDEX, NO: AE-1 } \\
\hline AUTHOR (s): & \multicolumn{3}{|c|}{$\begin{array}{l}\text { Duncan, L. M. } \\
\text { Gordon, W. E. }\end{array}$} & \multicolumn{2}{|c|}{. } \\
\hline \multicolumn{6}{|c|}{ AUTHOR AFFILIATION: Rice University } \\
\hline \multicolumn{6}{|c|}{ TITLE: Ionosphere/Microwave Beam Interaction Study } \\
\hline DATE: Septem & nber 1977 & VOLUMES: & PAGES: 117 & REFERENCES: & 9 \\
\hline ACQUISITION & No: & $\begin{array}{l}\text { NAS9-15212 } \\
\text { DRL No. T-1349 } \\
\text { DRD No. MA-183TA }\end{array}$ & & SOURCE: & 4 \\
\hline KEY WORDS: & \multicolumn{2}{|c|}{$\begin{array}{l}\text { Experiments } \\
\text { Frequency Scaling } \\
\text { Interference Effects }\end{array}$} & \multicolumn{3}{|c|}{$\begin{array}{l}\text { Heater Facilities } \\
\text { Ionosphere Heating } \\
\text { Ionosphere Predictions }\end{array}$} \\
\hline
\end{tabular}

\begin{tabular}{|c|c|c|c|c|c|}
\hline \multicolumn{4}{|c|}{ SUBJECT: ATMOSPHERIC EFFECTS } & \multicolumn{2}{|c|}{ SPSPO INDEX NO: AE-5 } \\
\hline $\operatorname{AUTHOR}(s):$ & \multicolumn{2}{|c|}{$\begin{array}{l}\text { Duncan, L. M. } \\
\text { Zinn, J. }\end{array}$} & & & \\
\hline \multicolumn{4}{|c|}{ Los Alamos Scientific Laboratory } & & \\
\hline TITLE: & \multicolumn{4}{|c|}{ Ionosphere-Microwave Interactions for Solar Power Satellite } & \\
\hline DATE: March & 1978 & VOLUMES & PAGES: 38 & REFERENCES : & 19 \\
\hline ACQUISITION & NO: & LA-UR-T & & SOURCE : & 5 \\
\hline KEY WORDS: & $\begin{array}{l}\text { Exper } \\
\text { Heati } \\
\text { Heati }\end{array}$ & $\begin{array}{l}\text { ents } \\
\text { Effects } \\
\text { Theory }\end{array}$ & $\begin{array}{l}\text { Ionosphere } H \\
\text { Ionosphere } \mathrm{P} \\
\text { Plasma Effec }\end{array}$ & $\begin{array}{l}\text { Heating } \\
\text { Predictions } \\
\text { cts }\end{array}$ & \\
\hline
\end{tabular}




$\begin{aligned} \operatorname{AUTHOR}(s): & \text { (1) Duncan, L. M. } \\ & \text { (2) Behnke, R. A. }\end{aligned}$

AUTHOR AFFILIATION: (1) Rice University

(2) National Astronomy and Ionospheric Center

TITLE: Observations of Self-Focusing Electromagnetic Waves in the Ionosphere

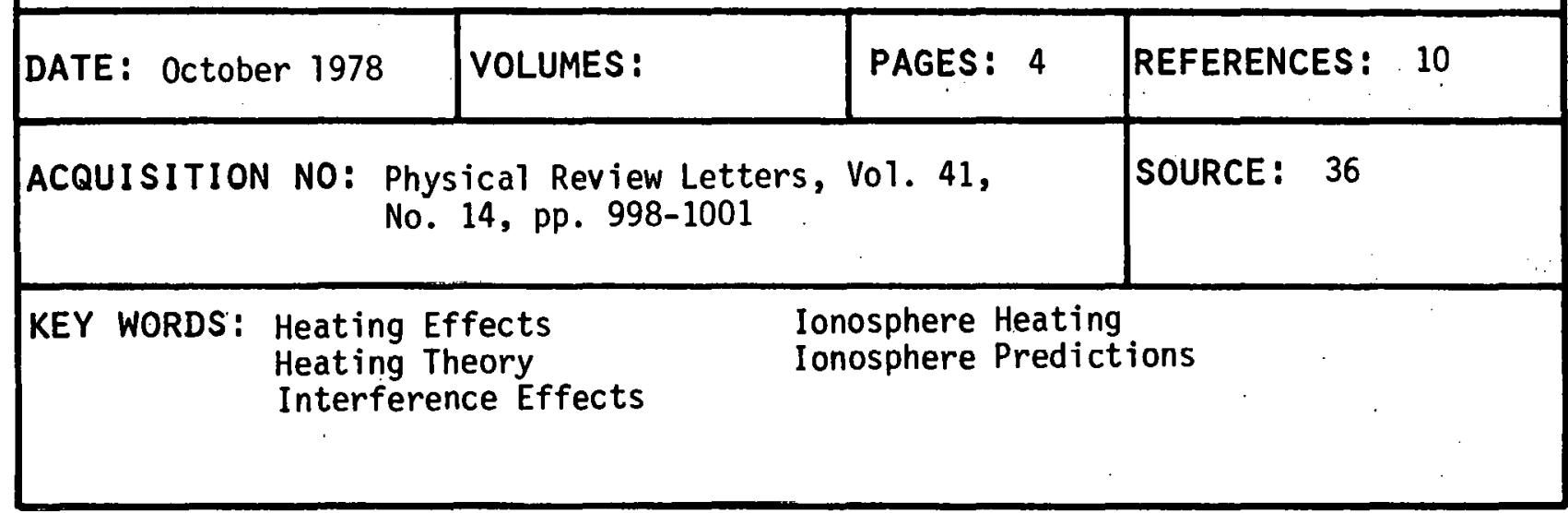

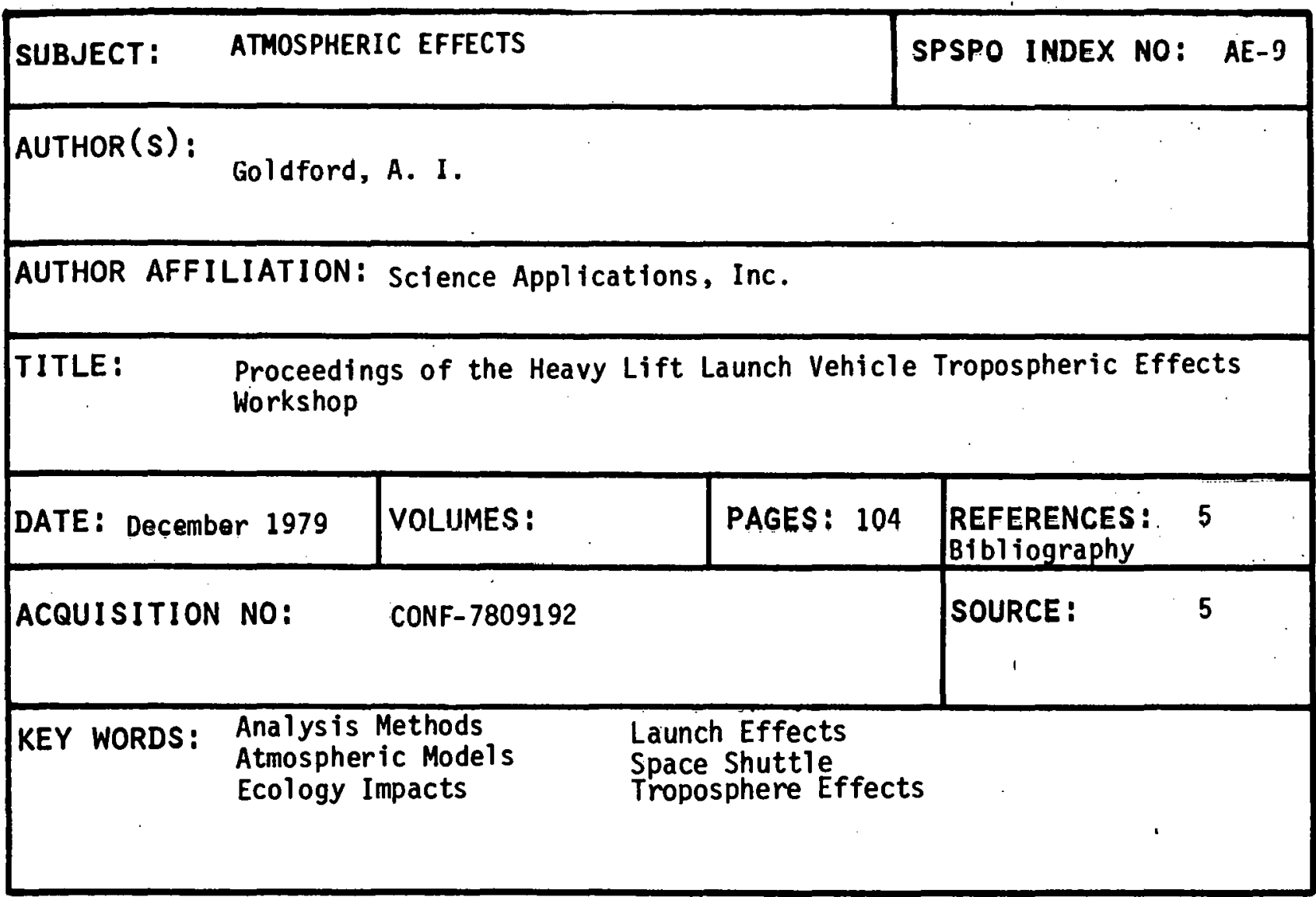


JTHOR (s): Gordon, W. E.

Duncan, L. M.

AUTHOR AFFILIATION: Rice University

TITLE: Ionosphere/Microwave Beam Interaction Study Statement of Work

\begin{tabular}{|l|l|l|lc|}
\hline DATE: JuTY 1978 & VOLUMES: & PAGES: 47 & REFERENCES: 35 \\
\hline ACQUISITION NO: & $\begin{array}{l}\text { NAS9-15212 } \\
\text { DRL No. T-1349 } \\
\text { DRD No. MA-183TA }\end{array}$ & SOURCE: \\
\hline
\end{tabular}

KEY WORDS: Experiments

Interference Effects

Ionosphere Predictions

Ionosphere Heating

Pilot Signal

Power Losses

SUBJECT: ATMOSPHERIC EFFECTS

SPSPO INDEX NO: AE-3

$\operatorname{AUTHOR}(s)$ : Johnson Space Center

AUTHOR AFFILIATION: National Aeronautics and Space Administration

TITLE: Proceedings of the Space Shuttle Environmental Assessmenl

Workshop on Stratospheric Effects

\begin{tabular}{|l|l|l|l|}
\hline DATE: September 1976 & VOLUMES: & PAGES: 213 & REFERENCES: 156 \\
\hline ACQUISITION NO: JSC-11633 & SOURCE: & 4 \\
\hline KEY WORDS: $\begin{array}{l}\text { Biologic Effects } \\
\text { Climate Effects } \\
\text { Particulate Exhausts }\end{array}$ & $\begin{array}{l}\text { Propellant Characteristics } \\
\text { Space Shuttle } \\
\text { Stratospheric Effects }\end{array}$ \\
\hline
\end{tabular}


AUTHOR (s): Lee, J.L.

AUTHOR AFFILIATION: Argonne National Laboratory.

TITLE: $\quad$ Proceedings of the Workshop on Meteorological Effects of Satellite Power System Rectenna Operation and Related Microwave Transmission Problems

\begin{tabular}{|l|l|l|l|}
\hline DATE: December 1979 & VOLUMES: & PAGES: 48 & REFERENCES: 16 \\
\hline ACQUISITION NO: & CONF-7808114 & 3OURCE: \\
& & & 5 \\
\hline
\end{tabular}

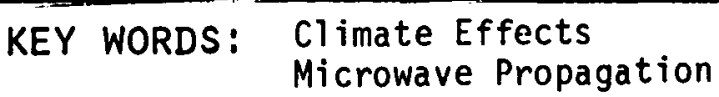

Rectenna Heating

Physical Processes

SUBJECT: ATMOSPHERIC EFFECTS

SPSPO INDEX NO: $A E-14$

AUTHOR (s): (1) Markson, R.

(2) Muir, M.

AUTHOR AFFILIATION:

(1) Massachusetts Institytc of Techno]ügy

TITLE :

Solar Wind Control of the Earth's Electric Field

\begin{tabular}{|l|l|l|l|}
\hline DATE: May 1980 & VOLUMES: & PAGES: 12 & REFERENCES: 90 \\
\hline ACQUISITION NO: $0036-8075 / 80 / 0530-0979$ & & SOURCE: 7 \\
\hline
\end{tabular}

KEY WORDS :

Climate Effects

Experiments

Physical Processes 


\begin{tabular}{r} 
AUTHOR (S): $\begin{array}{r}\text { Mendil110, M. (1) } \\
\text { Hermiter, B. (1) } \\
\text { Rote, D. M: }\end{array}$ \\
\hline AUTHOR AFFILIATION: \\
(1) Boston University \\
(2) Argonne National Laboratory
\end{tabular}

TITLE: Modification of the Aerospace Environment by Large Space Vehicles

\begin{tabular}{|c|c|c|c|}
\hline DATE: January 1979 & VOLUMES : & PAGES : 7 & REFERENCES : 17 \\
\hline \multicolumn{3}{|c|}{ ACQUISITION NO: $79-0391$} & SOURCE : 2 \\
\hline \multicolumn{2}{|c|}{$\begin{aligned} \text { KEY WORDS: } & \text { Exhaust Emmissions } \\
& \text { Physical Processes } \\
& \text { Satellite Power System }\end{aligned}$} & \multicolumn{2}{|c|}{ Transportation Effects } \\
\hline
\end{tabular}

SUBJECT: ATMOSPHERIC EFFECTS

SPSPO INDEX NO: $A E-21$

AUTHOR (s): Mendillo, M. (Ed.)

Baumgardner, B. (Ed.)

AUTHOR AFFILIATION: Boston University

TITLE: Proceedings of the Workshop/Symposium on the Preliminary Evaluation of the Ionospheric Disturbances Associated with the HEAO-C Launch, with Applications to the SPS Environmental Assessment.

DATE: August 1980

VOLUMES :

PAGES: 258

REFERENCES : 89

ACQUISITION NO: CONF-7911108

SOURCE: 5

KEY WORDS: Analys is Methods

Launch Effects

Experiments

Interference Effects 
AUTHOR(s): National Telecommunications and Information Administration

AUTHOR AFFILIATION: U. S. Department of Commerce

TITLE: Environmental Assessment for the Satellite Power System - Concept Development and Evaluation Program - Effects. of Ionospheric Heating on Telecommunications

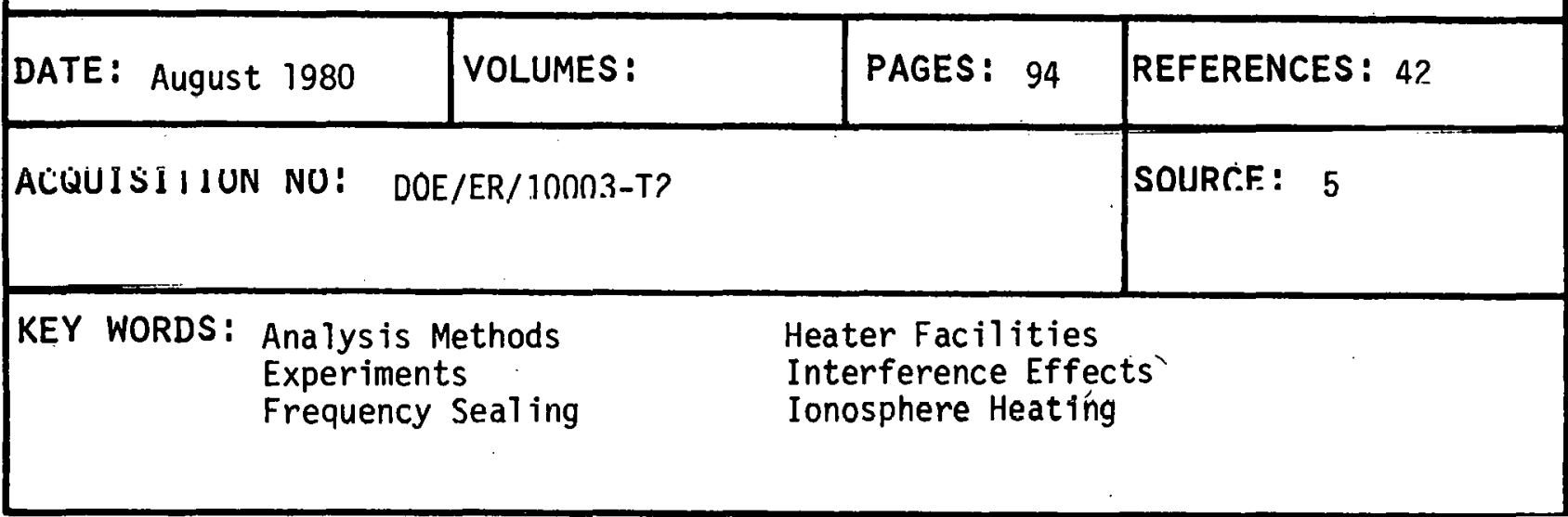

\begin{tabular}{|c|c|c|c|}
\hline \multicolumn{3}{|c|}{ SUBJECT: ATMOSPHERIC EFFECTS } & PSPO INDEX NO: AE-12 \\
\hline \multicolumn{2}{|l|}{$\operatorname{AUTHOR}(s):$} & \multicolumn{2}{|c|}{$\begin{array}{l}\text { Hegg, D. A. } \\
\text { Hobbs, P.V. }\end{array}$} \\
\hline \multicolumn{4}{|c|}{ AUTHOR AFFILIATION: University of Washington } \\
\hline \multicolumn{4}{|c|}{$\begin{array}{l}\text { TITLE: Measurements of the Particles and Gases in the Ground Cloud from an } \\
\text { Atlas/Centaur Rocket }\end{array}$} \\
\hline \multicolumn{2}{|c|}{ DATE: February, $1979 . \quad$ VOLUMES: } & PAGES: 70 & REFERENCES : 6 \\
\hline \multicolumn{3}{|c|}{ ACQUISITION NO: By Title. } & SOURCE: 5 \\
\hline $\begin{array}{c}\text { KEY WORDS: } \\
\text {. }\end{array}$ & $\begin{array}{l}\text { Climate Effects } \\
\text { Exhaust Emissions } \\
\text { Launch Effects }\end{array}$ & $\begin{array}{l}\text { Particulat } \\
\text { Propellant } \\
\text { Transporta }\end{array}$ & $\begin{array}{l}\text { Exhausts } \\
\text { Characteristics } \\
\text { ion Effects }\end{array}$ \\
\hline
\end{tabular}


AUTHOR(S): Rice University

AUTHOR AFFILIATION: Rice University

TITLE: Electrostatic Protection of the Solar Power Satellite and Rectenna; Part II-Lightning Protection of the Rectenna.

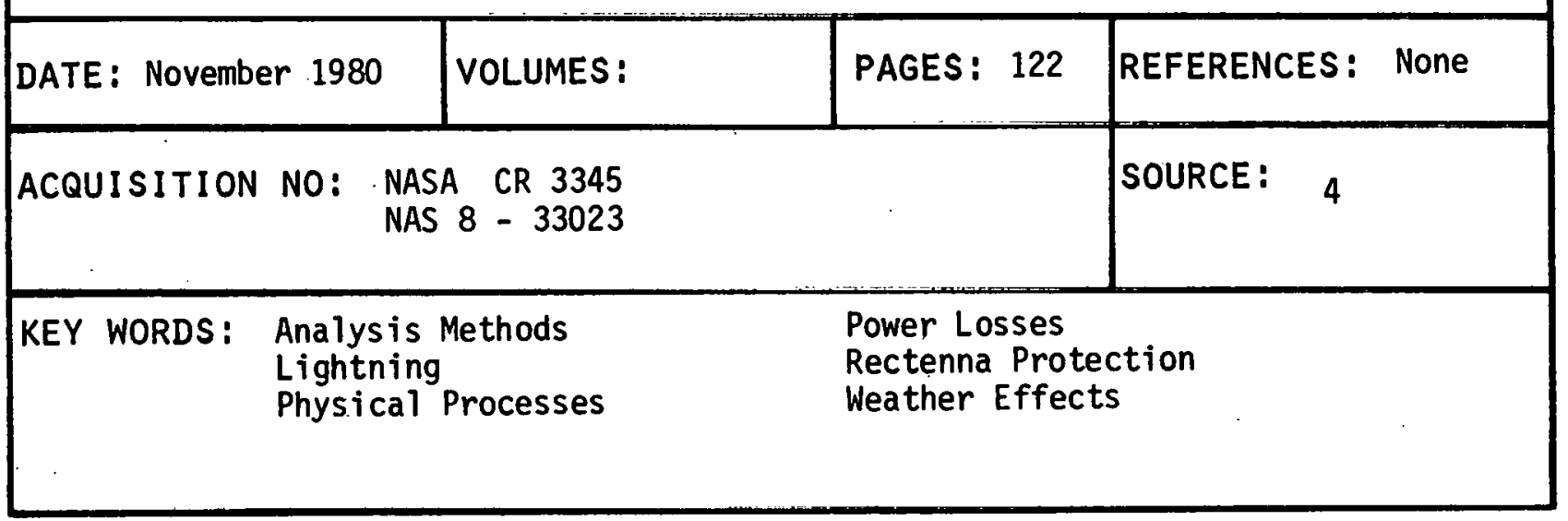

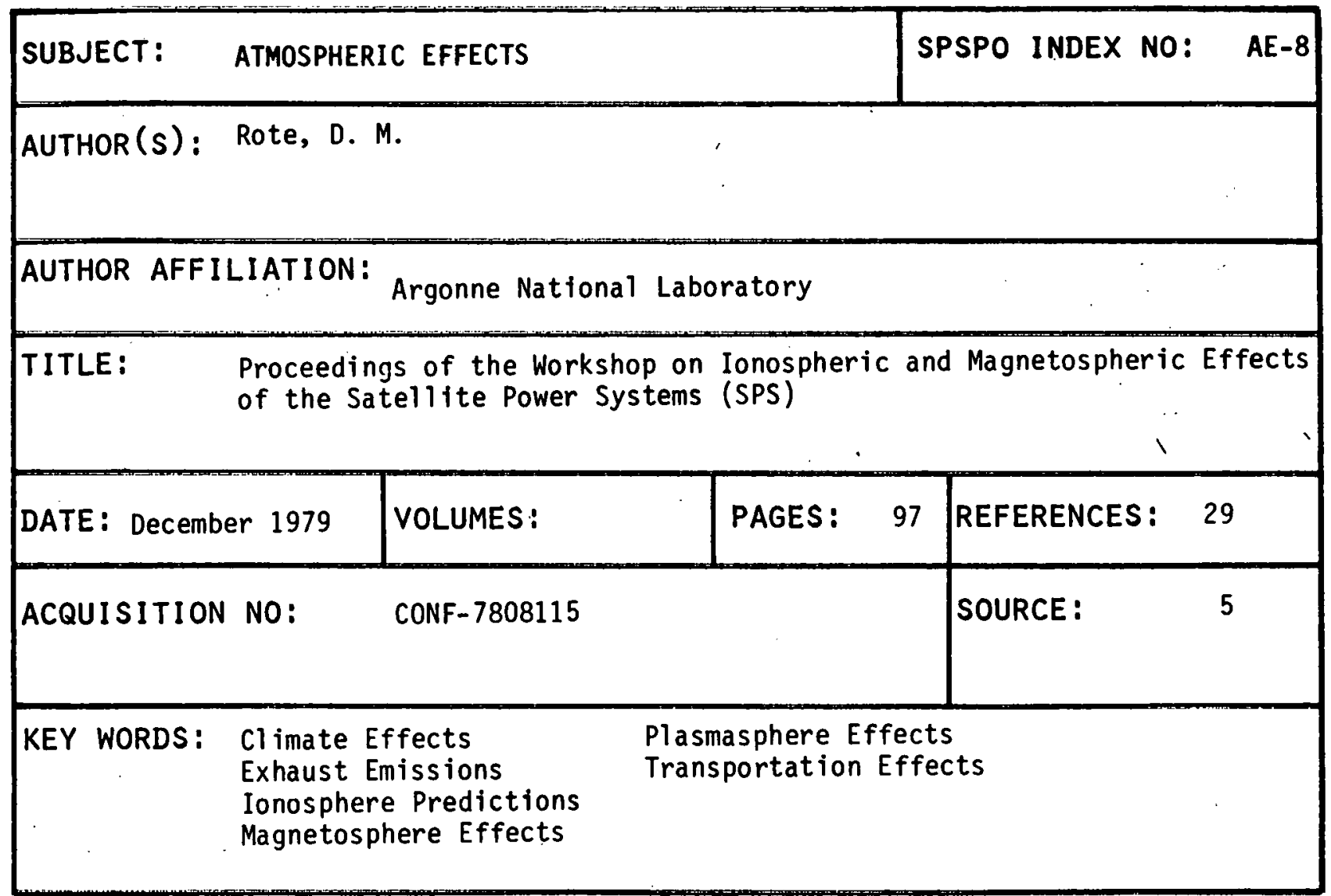




$\begin{array}{lll}\text { AUTHOR (S): } & \text { Rote, D. M. } & \text { Lee, J. L. } \\ & \text { Brubaker, K. L. } & \text { Valentino, A. R. }\end{array}$

\section{AUTHOR AFFILIATION: Argonne National Laboratory}

TITLE: An Assessment of the Atmospheric Effects of a Satellite Power System

\begin{tabular}{|l|l|l|l|}
\hline DATE: December 1979 & VOLUMES: & PAGES: 29 & REFERENCES: 36 \\
\hline ACQUISITION NO: By Title (Second Miami International \\
$\begin{array}{r}\text { Conference on Alternative } \\
\text { Energy Sources) }\end{array}$ & SOURCE: 37 \\
\hline
\end{tabular}
KEY WORDS:
Atmospheric Models
Launch Effects
Climate Effects
Physical Processes
Exhaust Emissions
Transportation Effects

\section{SUBJECT: ATMOSPHERIC EFFECTS

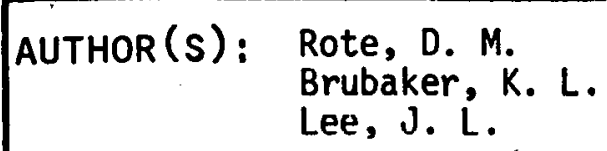

SPSPO INDEX NO: $A E-27$

AUTHOR AFFILIATION: Argonne National Laboratory

TITLE: Environmental Assessment for the Satellite Power System - Concept Development and Evaluation Program - Atmospheric Effects

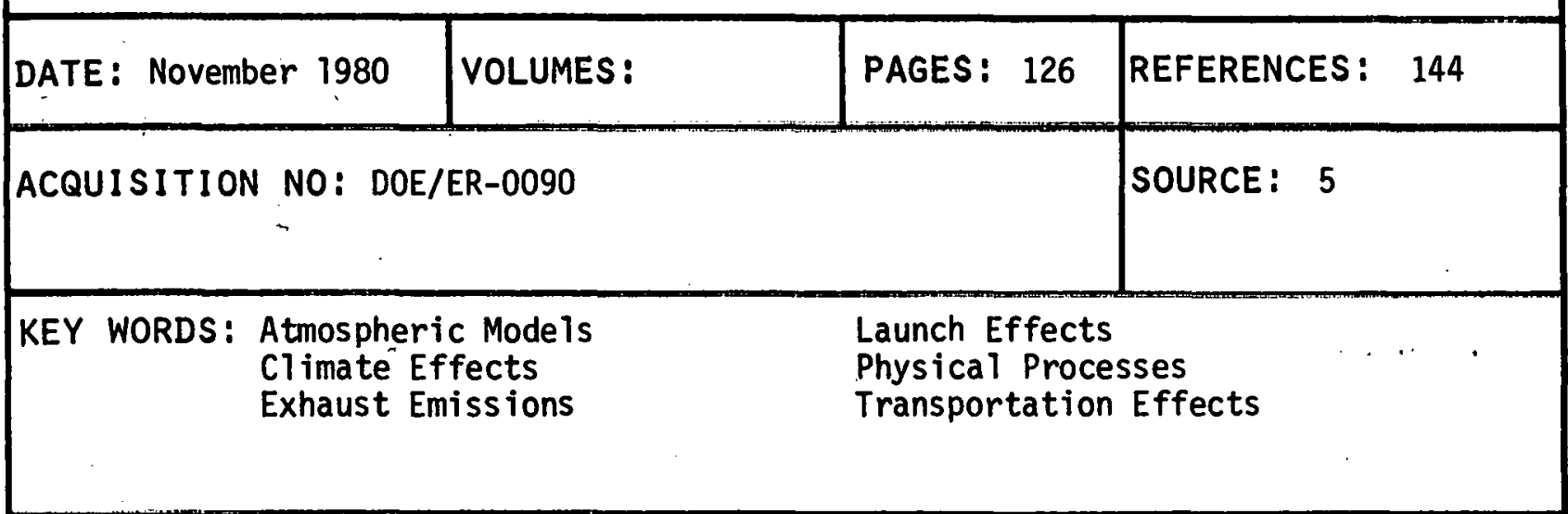




\section{SUBJECT:}

ATMOSPHERIC EFFECTS

$\operatorname{AUTHOR}(s)$ : Rush, C. M.

Violette, E. J.

Carroll, J. C.

Espeland, R. H.

Allen, K. C.

AUTHOR AFFILIATION: U. S. Department of Commerce

TITLE: Impact of SPS Heating on VLF, LF, and MF Telecommunications. Systems Ascertained by Experimental Means

DATE: JUTY 1980

VOLUMES :

PAGES : 105

REFERENCES : 15

ACQUISITION NO: DOE/ER/10003-TI

SOURCE： 5

KEY WORDS: Analysis Methods

Experiments

Frequency Scaling

Heating Effects

Interference Effects

Ionosphere Heating

SUBJECT: ATMOSPHERIC EFFECTS

SPSPO INDEX NO: AE-26

AUTHOR (S): Showen, R. L.

AUTHOR AFFILIATION: SRI International

TITLE: Measurements of Possible D- and E- Region Telecormunications Effects During Ionospheric Heating

DATE: November 1980

VOLUMES:

PAGES: 38

REFERENCES: None

ACQUISITION NO: Contract NT79RAC96007

SOURCE : 13

KEY WORDS: Heating Effects

Heating Theory

Interference. Effects

Ionosphere Heating

Ionosphere Predictions 


$$
\begin{array}{r}
\text { AUTHOR (s): Zinn, J. } \\
\text { Sutherland, C. D. }
\end{array}
$$

AUTHOR AFFILIATION: LOS Alamos Scientific Laboratory

TITLE: Effects of Rocket Exhaust Products in the Thermosphere and Ionosphere

\begin{tabular}{|c|c|c|c|}
\hline \multicolumn{3}{|c|}{ SUBJECT: ATMOSPHERIC EFFECTS } & SPSPO INDEX NO: AE-13 \\
\hline \multicolumn{4}{|c|}{$\begin{array}{ll}\operatorname{AUTHOR}(s): & \text { Zinn, J. } \\
& \text { Sutherland, C. D. }\end{array}$} \\
\hline \multicolumn{4}{|c|}{ AUTHOR AFFILIATION: Los Alamos Scientific Laboratory } \\
\hline \multicolumn{4}{|c|}{$\begin{array}{l}\text { TITLE: Effects of Rocket Exhaust Products in the Thermosphere } \\
\text { and Ionosphere }\end{array}$} \\
\hline DATE: February 1980 & VOLUMES: & PAGES: 22 & REFERENCES: 20 \\
\hline \multicolumn{3}{|c|}{ ACQUISITION NO: LA-8233-MS } & SOURCE: 5 \\
\hline \multicolumn{2}{|c|}{$\begin{array}{l}\text { KEY WORDS: Analys is Methods } \\
\text { Atmospheric Models } \\
\text { Exhaust Emissions }\end{array}$} & \multicolumn{2}{|c|}{$\begin{array}{l}\text { Ionosphere Predictions } \\
\text { Launch Effects } \\
\text { Transportation Effects }\end{array}$} \\
\hline
\end{tabular}

\begin{tabular}{|l|l|l|l|}
\hline DATE: 1980 & VOLUMES: & PAGES: 24 & REFERENCES: 20 \\
\hline ACQUISITION NO: $\begin{array}{l}\text { 0191-9067/80/010109-24 } \\
\text { Space Solar Power Review, Vol. 1, } \\
\text { pp. 109-132 }\end{array}$ & SOURCE : 33 \\
\hline KEY WORDS: $\begin{array}{l}\text { Atmospheric Models } \\
\text { Exhaust Emissions } \\
\text { Experiments }\end{array}$ & $\begin{array}{l}\text { Ionosphere Heating } \\
\text { Physical Processes } \\
\text { Transportation Effects }\end{array}$ \\
\hline
\end{tabular}


AUTHOR (s) :

(1) Zinn, J.

(1) Sutherland, C. D.

(1) Stone, S. N.

(1) Duncan, L. M.

(2) Behnke, R. A.

AUTHOR AFFILIATION:

(1) Los Alamos Scientific Laboratory

(2) Arecibo Observatory

TITLE: Ionospheric Effects of Rocket Exhaust Products

(HEAO-C, Skylab and SPS-HLLV)

DATE: October 1980

VOLUMES :

PAGES: 32

REFERENCES : 28

ACQUISITION NO: DOE/ER-0082

SOURCE : 5

KEY. WORDS: Atmospheric Models

Ionosphere Predictions

Exhaust Emissions

Physical Processes

Experiments

Transportation Effects 


\section{THIS PAGE WAS INTENTIONALLY LEFT BLANK}




\section{BIOLOGIC EFFECTS \\ (CATEGORY BE)}

Document in this category cover potential biologic effects which might be produced by solar energy or SPS microwave energy in space or on earth, and biologic effects which might be produced by SPS launch and space vehicles, electric transmission lines, or SPS-related industrial and manufacturing processes. Health, safety and other biologic effects (e.g., physiological, behavioral, etc.) on humans, animals and plants are included. Biologic and occupational safety issues related to working in the space ionizing radiation environment are included. They describe and evaluate what is known about the radiation environment in space, its effects on humans and space equipment, and possible ameliorative measures which may be useful in controlling such effects.

KEY WORDS:

Avian Species

Behavior Effects

Blood-Brain Barrier

Charged Particles

Cosmic Rays

Data Review

Dose Levels

Dosimetry

Electron Flux

Experimental Data

Exposure

Extremely Low Frequency

tye Hazards

Geophysics

Growth and Development

Health Risks

Human Absorption

Infrared

Insects

Ionizing Radiation

Lasers
PAGE NUMBER:

$34,36,46$

$23,33,34,35,36,37,39$

$24,38,47$

28,41

41,49

$24,25,26,32,33,35,36,37,38,39$,

$40,42,44,45,46,47,49$

41,49

$24,30,31,34,36,37,39,41,43,48$

49

$23,34,35,36,46,47,50$

$23,26,27,29,33,35,36,37,41,43$, $44,46,47,48$

$24,34,38,42,46,47,48$

49 ,

28

23,49

41,44

$32,35,36,40,41,43,47,50$

37

23,35

$26,27,28,44$

38 
(Biologic Effects

Category $B E$ continued)

KEY WORDS:

Mammal Studies

Measurement Methods

Measurements

Microwave

Mitigating Strategy

Occupational Exposure

Proton Flux

Public Concern

Public Health

Radiation Exposure

Reflected Light

Regulation

Reptiles

Research

Shielding

Solar Effects

Solar Flares

Space Flight

Space Ins truments

Space Lab

Space Workers

Specific Absorption

Standards

Theory

Thermal Effects

Transmission Line

Ultrasonics

Ultraviolet.

Vegetation Effects

Zero Gravity

RELATED CATEGORIES:

Atmospheric Effects (AE)

Environmental Effects (EE)

Power Generation and Transmission (PT)
PAGE NUMBER:

$35,37,38,39,46,49$

$24,32,34,36,37,38,3 \overline{9}, 4<, 44,50$

27

$23,24,25,26,27,28,29,30,31,32$,

$33,35,38,39,40,41,42,43,45,47,48,50$

$39,45,46$

$23,24,25,26,27,28,29,33,35,37,39,41$ $44,45,48$

49

$25,26,30,31,43,47,48$

$24,25,26,27,28,29,30,31,32,33,34,37$

$38,40,41,43,44,45,48$

$28,41,44$

49

$25,26,27,28,29,32,33,42,45,46,48$

34

$23,24,26,27,30,31,32,33,34,35,36,37$

$39,40,42,43,44,50$

28,41

37

49

39

28

49

39

$34,36,45$

$23,27,28,29,30,31,32,33,38,42,43,45$,

$23,25,32,35,36,40,42,43,47$

$25,34,47$.

$34,46,47$

38

37

46,49

39

Systems Analyses (SA)

Social Issues (SI)

Space Transportation (ST) 
AUTHOR (S): Gary, N. E.
$\quad$ Westerdahl, B. B.

AUTHOR AFFILIATION: University of California

TITLE: Environmental Assessment for the Satellite Power System (SPS): Studies of Honey Bees Exposed to $2.45 \mathrm{GHz}$ Continuous-Wave Electromagnetic Energy

\begin{tabular}{|c|c|c|c|}
\hline DATE: December 1980 & VOLUMES : & PAGES: 149 & REFERENCES : 55 \\
\hline \multicolumn{3}{|c|}{ ACQUISITION NO: DOE/ER-0095 } & SOURCE: 5 \\
\hline \multicolumn{2}{|c|}{$\begin{aligned} \text { KEY WORDS: } & \text { Behavior Effects } \\
& \text { Experimental Data } \\
& \text { Growth and Development }\end{aligned}$} & \multicolumn{2}{|c|}{$\begin{array}{l}\text { Insects } \\
\text { Microwave } \\
\text { Research }\end{array}$} \\
\hline
\end{tabular}

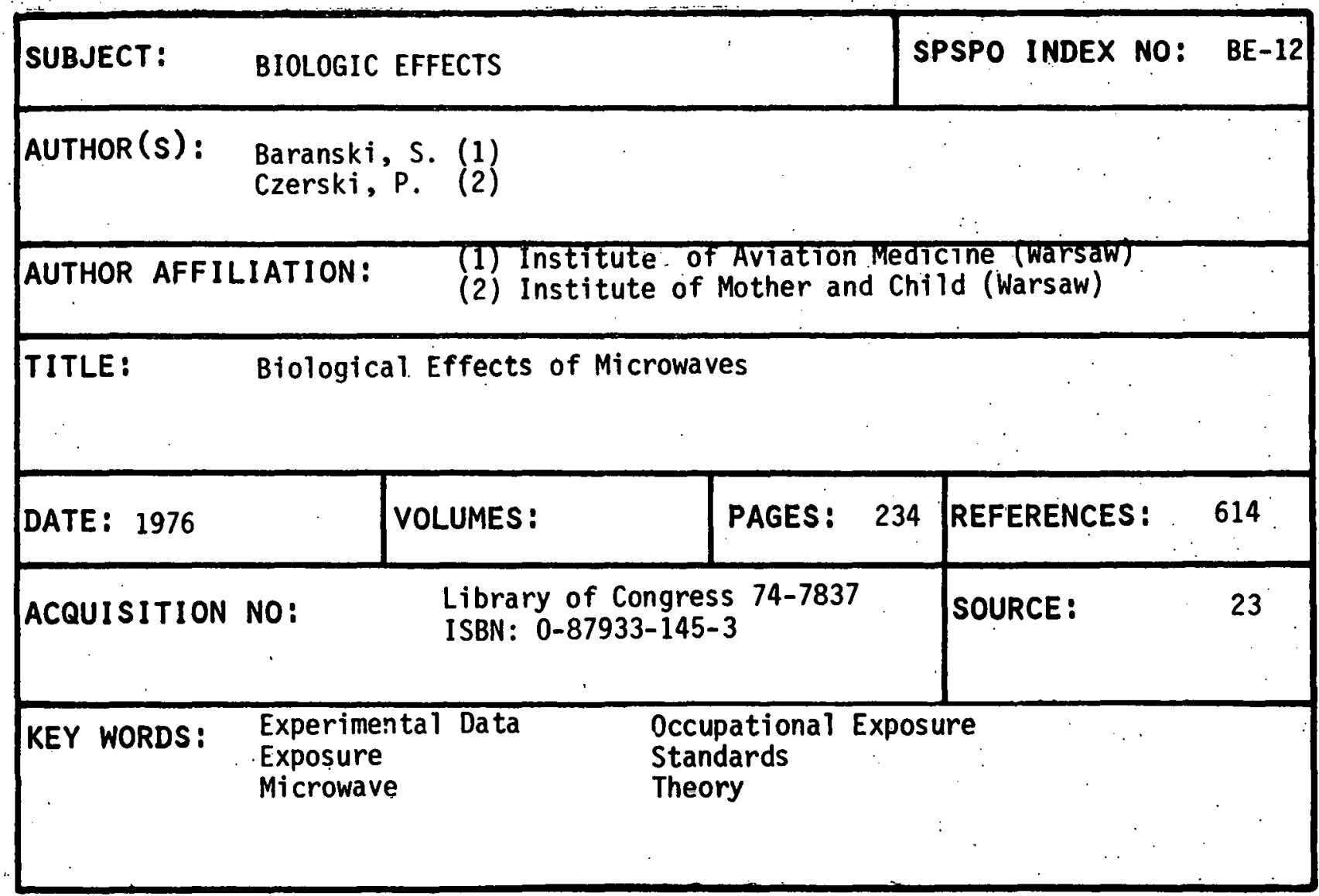




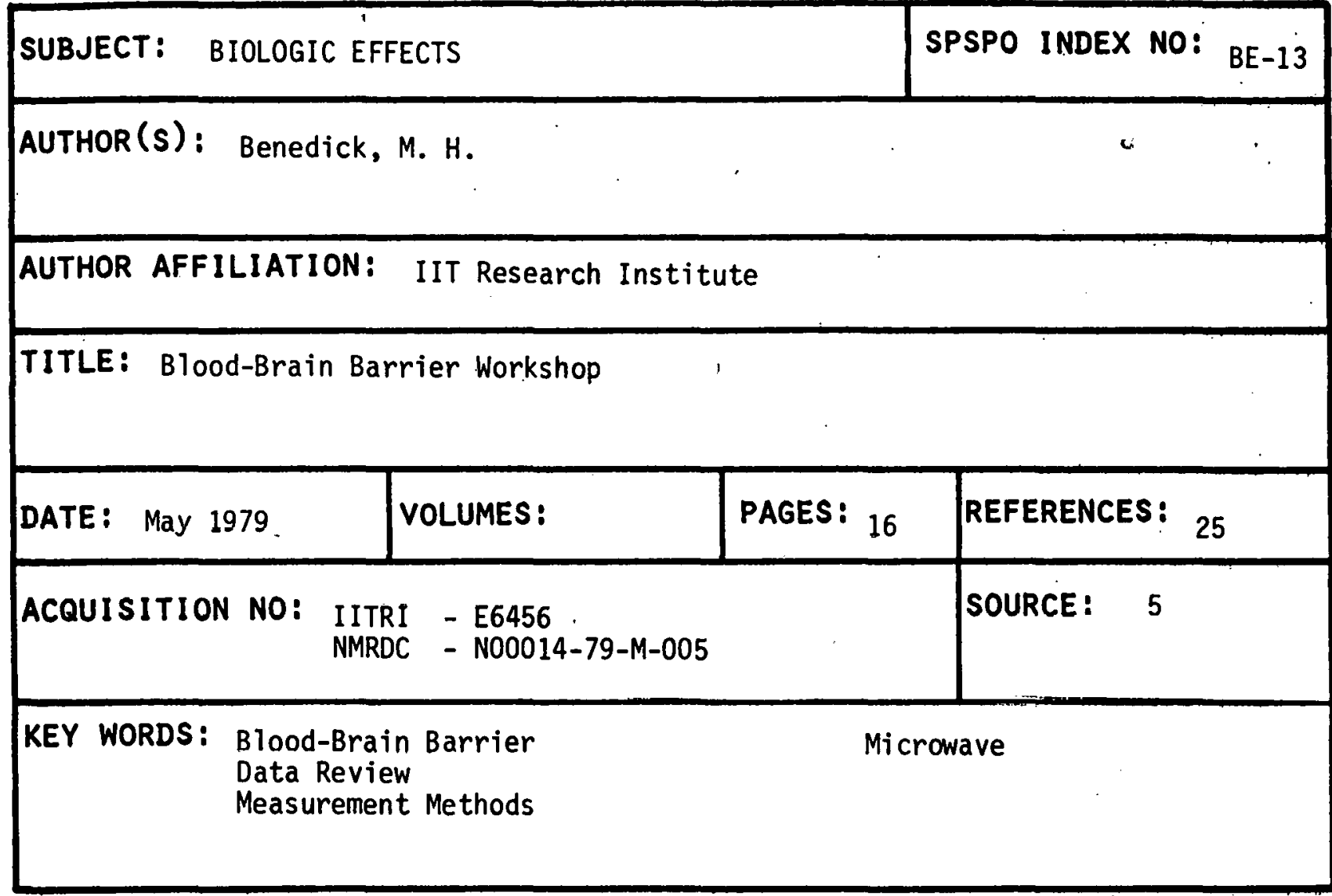

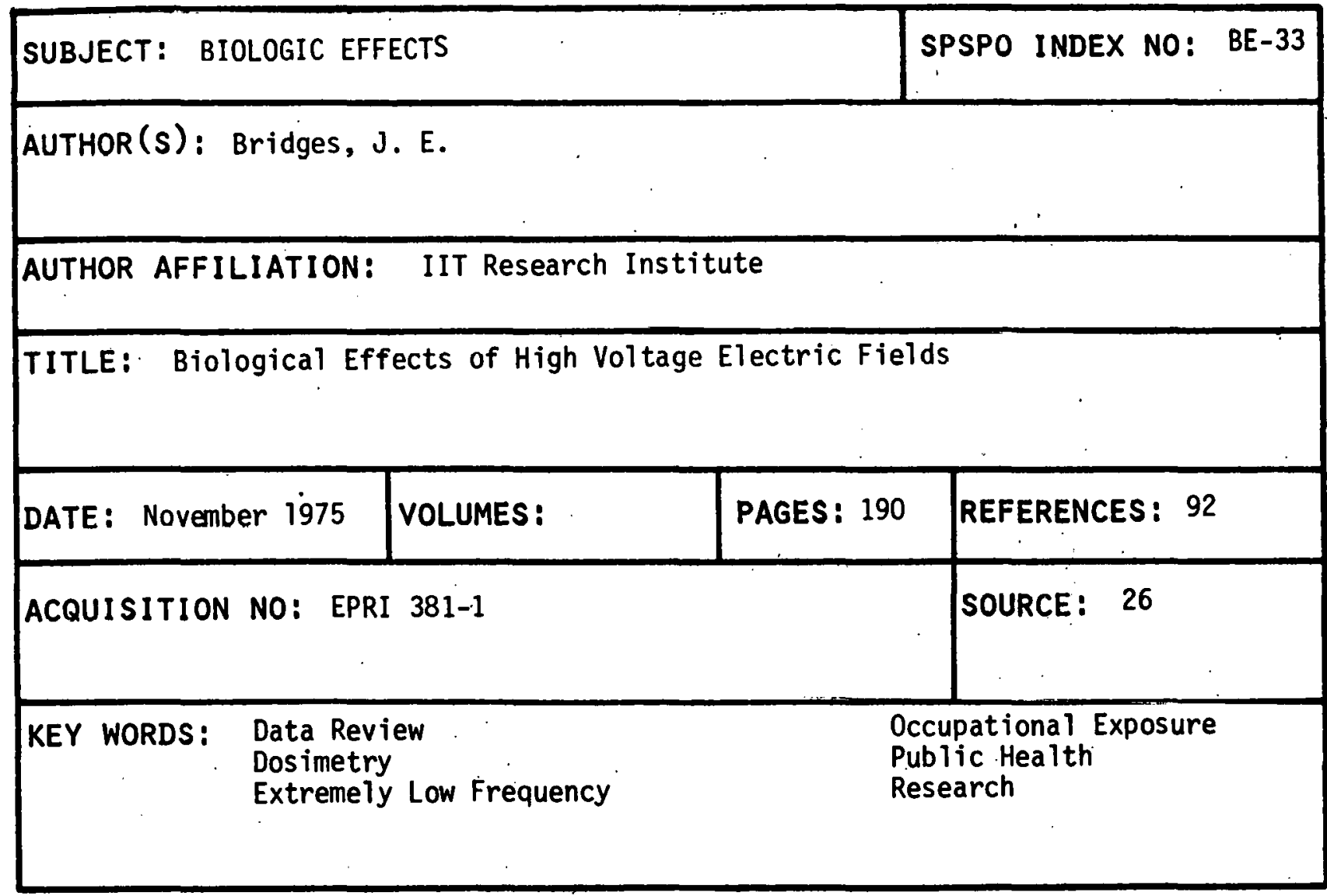


AUTHOR (s): Brodeur, P.

AUTHOR AFFILIATION: New Yorker Magazine

TITLE: The Zapping of America

\begin{tabular}{|c|c|c|c|}
\hline DATE: 1977 & VOLUMES: & PAGES: 343 & REFERENCES: 75 \\
\hline \multicolumn{3}{|c|}{ ACQUISITION NO: By Title } & SOURCE: 32 \\
\hline KEY WORDS: & $\begin{array}{l}\text { Data Review } \\
\text { Microwave } \\
\text { Occupational Exposure }\end{array}$ & $\begin{array}{l}\text { Public Concern } \\
\text { Public Health } \\
\text { Regulation }\end{array}$ & \\
\hline
\end{tabular}

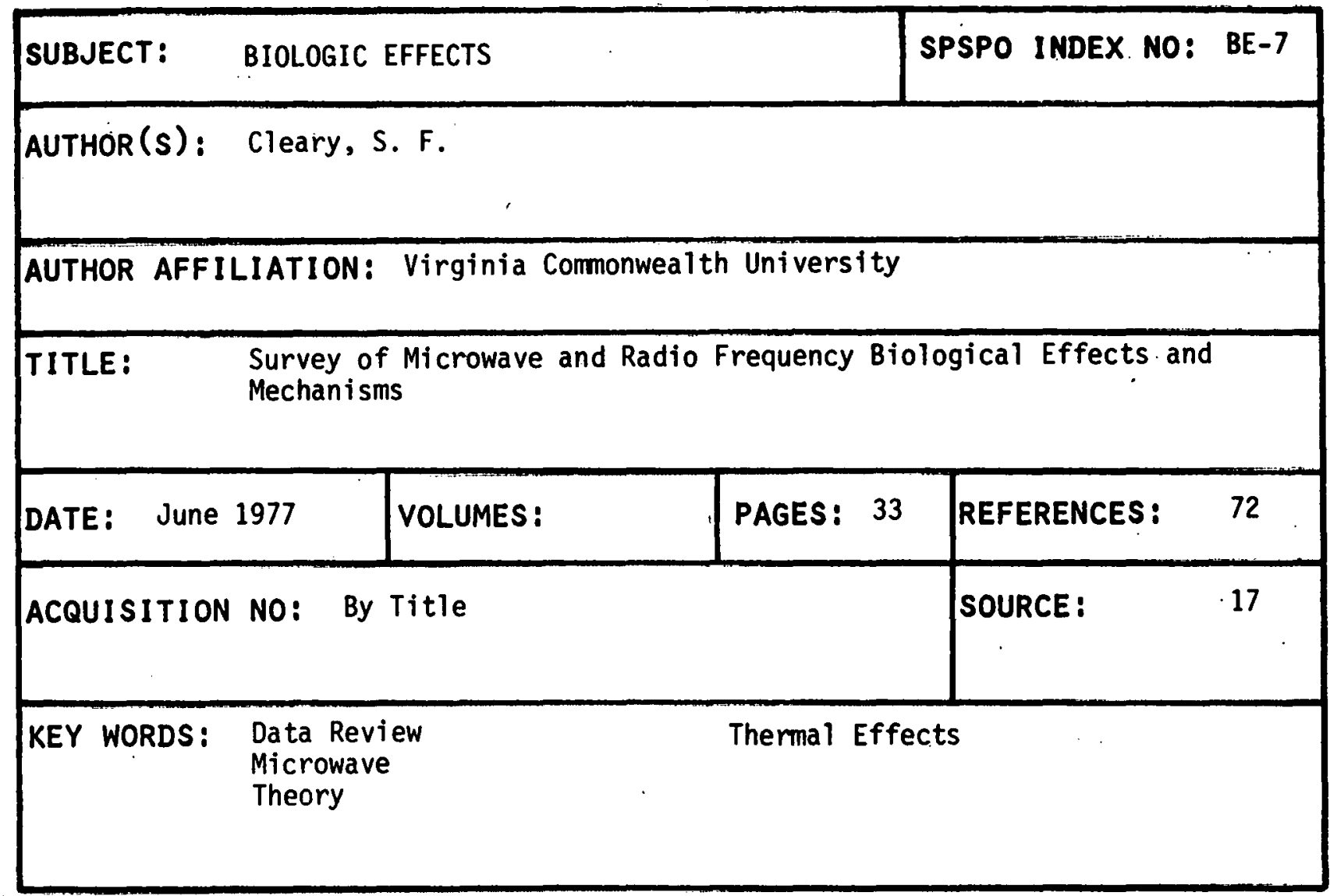




\section{SUBJECT: BIOLOGIC EFFECTS}

AUTHOR(s): Committee on Commerce, Science and Tran'sportation

AUTHOR AFFILIATION: U. S. Senate

TITLE: Report on Radiation Health and Safety

\begin{tabular}{|l|l|l|l|}
\hline DATE: December 1978 & VOLUMES: & PAGES: 106 & REFERENCES: None \\
\hline ACQUISITION NOI 34949 & SOIIRCE: 20 \\
\hline KEY WORDS: & $\begin{array}{l}\text { Ionizing Radiation } \\
\text { Microwave } \\
\text { Occupational Exposure }\end{array}$ & $\begin{array}{l}\text { Public Health } \\
\text { Regulation } \\
\text { Research }\end{array}$ \\
\hline
\end{tabular}

SUBJECT: BIOLOGIC EFFECTS

AUTHOR(s): Committee on Commerce, Science and Transportation

AUTHOR AFFILIATION: United States Senate

TITLE: Microwave Irradiation of the U. S. Embassy in Moscow

\begin{tabular}{|l|l|l|l|}
\hline DATE: April 1979 & VOLUMES: & PAGES: 26 & REFERENCES: None \\
\hline ACQUISITION NO: 96USC-43-949 & SOURCE: 20 \\
\hline KEY WORDS: $\begin{array}{l}\text { Data Review } \\
\text { Exposure } \\
\text { Microwave }\end{array}$ & $\begin{array}{l}\text { Occupational Exposure } \\
\text { Public Concern }\end{array}$ \\
\hline
\end{tabular}


AUTHOR(s): Committee on Governmental Affairs

AUTHOR AFFILIATION: U. S. Senate

TITLE: Federal Regulation of Radiation Health and Safety: Organizational Problems and Possible Remedies

\begin{tabular}{|l|l|l|l|}
\hline DATE: August. 1978 & VOLUMES: & PAGES: 180 & REFERENCES: None \\
\hline ACQUISITION NO: $31-471-0$ & SOURCE: 20 \\
\hline KEY WORDS: $\begin{array}{l}\text { Exposure } \\
\text { Ionizing Radiation } \\
\text { Microwave }\end{array}$ & $\begin{array}{l}\text { Occupational Exposure } \\
\text { Public Health } \\
\text { Regulation }\end{array}$ \\
\hline
\end{tabular}

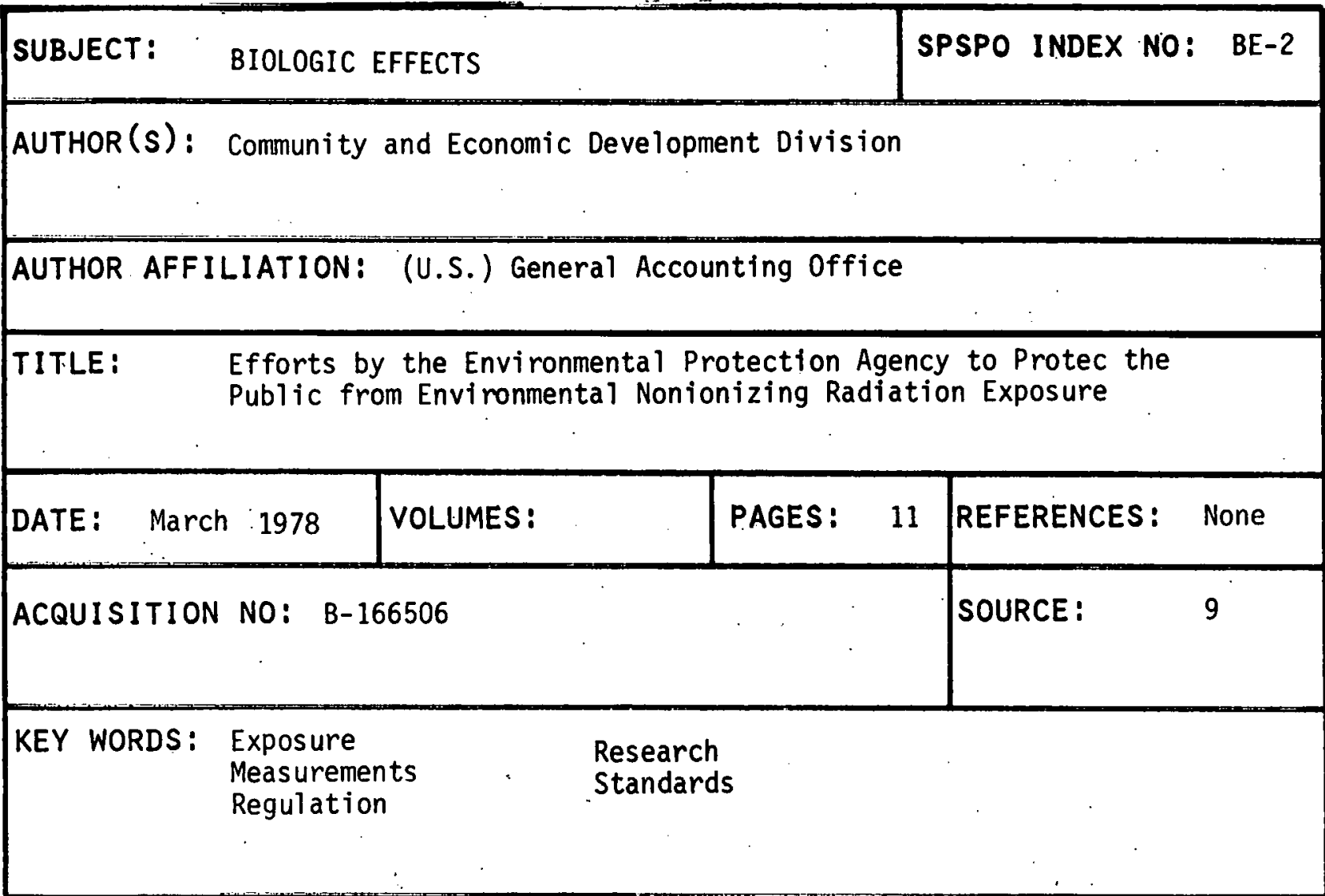


SUBJECT: BIOLOGIC EFFECTS

AUTHOR(S): Comptroller General of the U. S.

AUTHOR AFFILIATION: U. S. General Accounting Office

TITLE: More Protection from Microwave Radiation Hazards Needed

\begin{tabular}{|l|l|l|}
\hline DATE: November 1978 & VOLUMES: & PAGES: 80 \\
\hline
\end{tabular}

REFERENCES : Bibliography

ACQUISITION NO: HRD $=79=7$

SOURCE : 9

KEY WORDS: $\begin{array}{ll}\text { Microwave } & \text { Regulation } \\ \text { Occupational Exposure } & \text { Standards }\end{array}$

Public Health

SUBJECT:- BIOLOGIC EFFECTS

SPSPO INDEX NO: BE-52

AUTHOR(s): Curtis, S. B.

Schimerling, W.

AUTHOR AFFILIATION: Lawrence Berkeley Laboratory

TITLE: Workshop on the Radiation Environment of the Satellite Power System

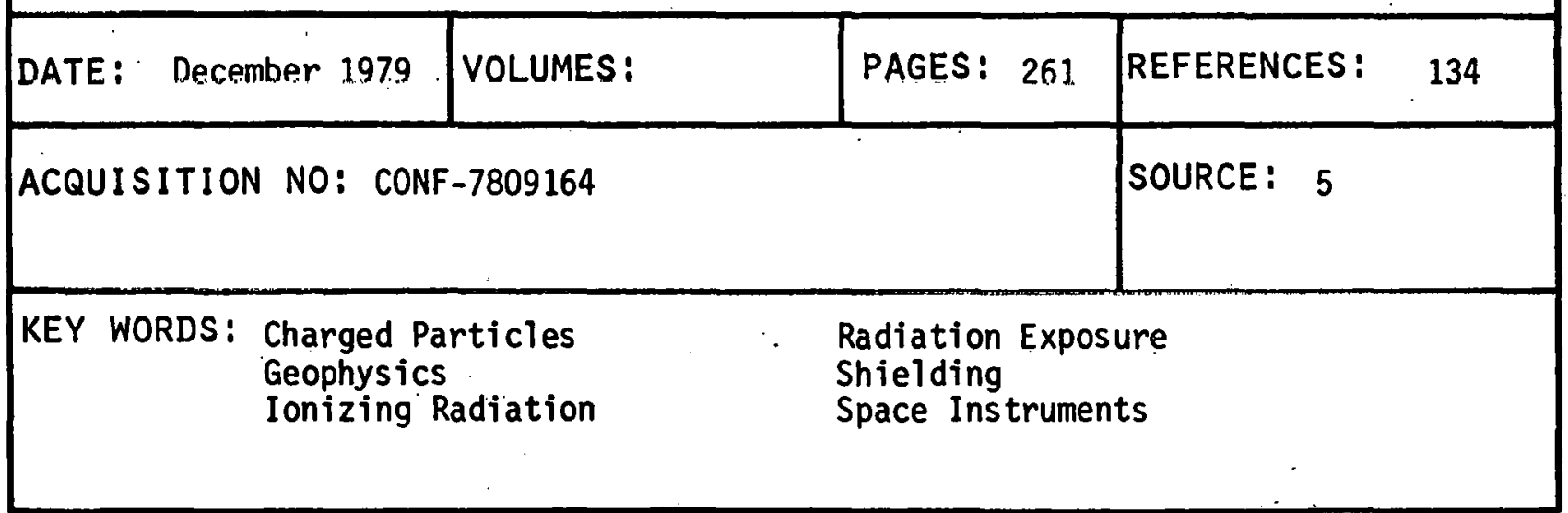




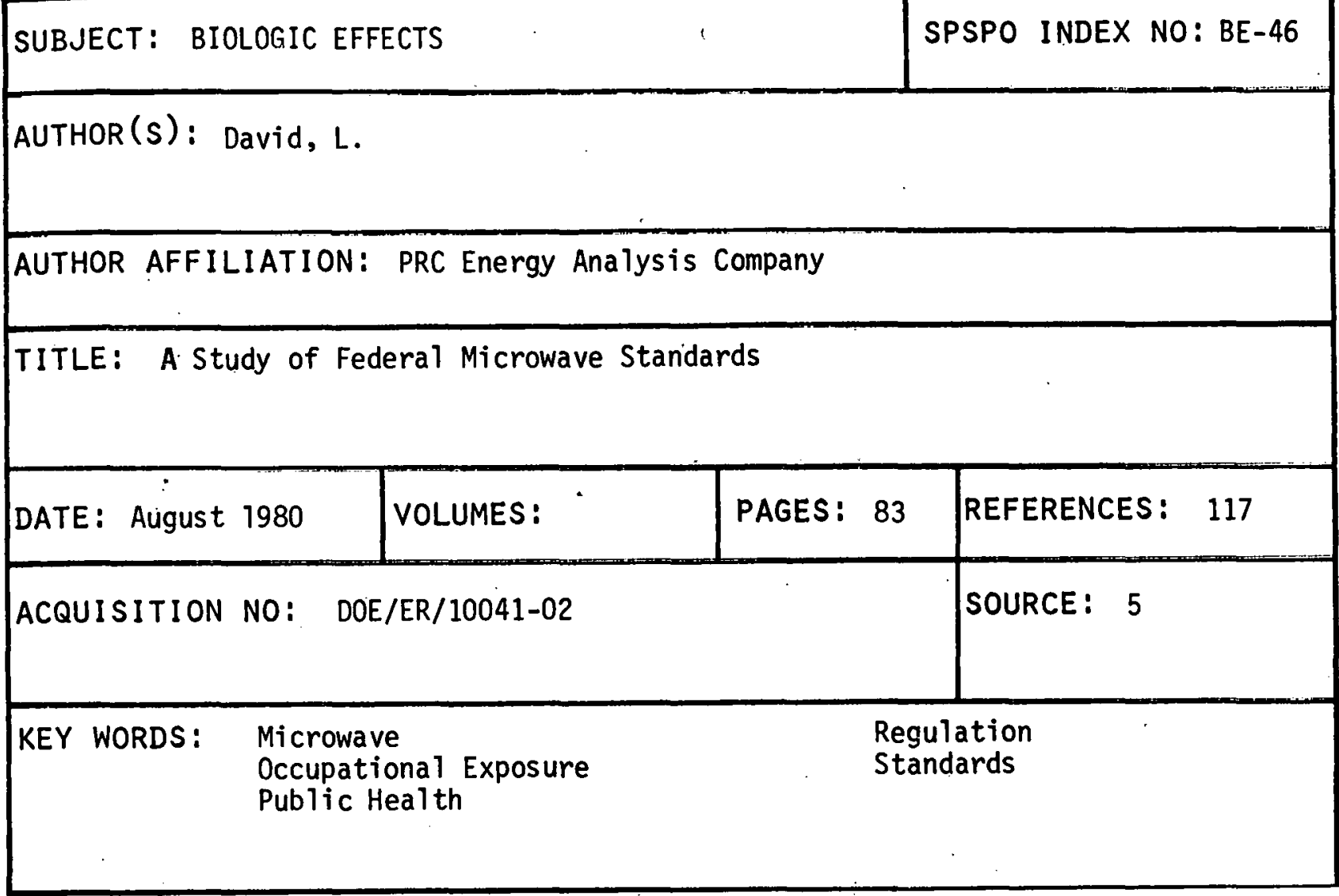

\section{SUBJECT: BIOLOGIC EFFECTS}

SPSPO INDEX NO: BE-1

AUTHOR(S): Dula, A.

AUTHOR AFFILIATION: Butler Binion Rice Cook and Knapp

TITLE: Microwave Radiation

\begin{tabular}{|l|l|l|lc|}
\hline DATE: 1978 & VOLUMES: & PAGES: 17 & REFERENCES: 41 \\
\hline ACQUISITION NO: Jurimetrics Journal, Summer 1978 & SOURCE: & 8 \\
pp. 2.91-3n7 & & \\
\hline
\end{tabular}

KEY WORDS: EXposure Regulation

Standards 
AUTHOR(s): Electromagnetic Radiation Management Advisory Council

AUTHOR AFFILIATION: Office of Telecommunications Policy

TITLE: Program for Control of Electromagnetic Pollution of the Environment: The Assessment of Bilogical Hazards of Nonionizing Electromagnetic Radiation

DATE: December 1971 VOLUUMES:

PAGES: 42 REFERENCES : 9

ACQUISITION NO: By Titlo SOURCE: 5

KEY WORDS: Dos imetry

Microwave

Public Concern
Public Health

Research

Standards

\section{SUBJECT: BIOLOGIC EFFECTS}

AUTHOR(S): Electromagnetic Radiation Management

Advisory Council

\section{AUTHOR AFFILIATION: Office of Telecommunications Policy}

TITLE: Program for Control of Electromagnetic Pollution of the Environment: The Assessment of Blological Hazards of Nonionizing Electromlayrietic Radiation

\begin{tabular}{|l|l|l|l|}
\hline DATE: March 1973 & VOLUMES: & PAGES: 44 & REFERENCE \\
\hline ACQUISITION NO: By Title & SOURCE: \\
\hline KEY WORDS: $\begin{array}{l}\text { Dosimetry } \\
\text { Microwave } \\
\text { Public Concern }\end{array}$ & $\begin{array}{l}\text { Public Health } \\
\text { Research } \\
\text { Standards }\end{array}$ \\
\hline
\end{tabular}


AUTHOR (S): Electromagnetic Radiation Management

Advisory Counci 1

AUTHOR AFFILIATION: Office of Telecommunications Policy

TITLE: Program for Control of Electromagnetic Pollution of the Environment:

The Assessment of Biological Hazards of Nonionizing Electromagnetic

Radiation.

DATE: May 1974

VOLUMES :

PAGES: 45 REFERENCES: 105

ACQUISITION NO: By Title

SOURCE： 5

KEY WORDS: Dosimetry

Microwave

Public Health

Public Concern

Research

Standards

SUBJECT: BIOLOGIC EFFECTS

SPSPO INDEX NO: BE-23

AUTHOR(S): Electromagnetic Radiation Management Advisory Council

AUTHOR AFFILIATION: Office of Telecommunications Policy

TITLE: Program for Control of Electromagnetic Pollution of the Environment: The Assessment of Biological Hazards of Nonionizing Electromagnetic Radiation.

\begin{tabular}{|c|c|c|c|c|c|}
\hline \multicolumn{2}{|c|}{ DATE: $\quad$ April 1975} & VOLUMES: & \multirow[t]{2}{*}{ PAGES: 32} & \multirow{2}{*}{\multicolumn{2}{|c|}{ REFERENCES : $\quad 245$}} \\
\hline \multicolumn{3}{|c|}{ ACQUISITION NO: By Title } & & & \\
\hline \multicolumn{3}{|c|}{$\begin{aligned} \text { KEY WORDS: } & \text { Dosimetry } \\
& \text { Microwave } \\
& \text { Public Concern }\end{aligned}$} & $\begin{array}{l}\text { Public Health } \\
\text { Research } \\
\text { Standards }\end{array}$ & . & \\
\hline
\end{tabular}


AUTHOR(S): Electromagnetic Radiation Management Advisory Council

AUTHOR AFFILIATION: Office of Telecommunications Policy

TITLE: Program for Control of Electromagnetic Pollution of the Environment:

The Assessment of Biological Hazards of Nonionizing Electromagnetic

Radiation

DATE: June 1976

VOLUMES :

PAGES: 213

REFERENCES : Bibliography

ACQUISITION NO: By TitTe SOURCE : 5

\begin{tabular}{lll}
\hline KEY WORDS: & $\begin{array}{l}\text { Data Review } \\
\text { Microwave } \\
\text { Public Heal th }\end{array}$ & $\begin{array}{l}\text { Regulation } \\
\text { Research } \\
\text { Standards }\end{array}$ \\
&
\end{tabular}

SUBJECT: BIOLOGIC EFFECTS

SPSPO INDEX NO: BE-32

AUTHOR(S): Environmental Health Directorate

AUTHOR AFFILIATION: Minister of National Health and Welfare (Canada)

TITLE: Health Aspects of Radio Frequency and Microwave Radiation Exposure Part 1.

\begin{tabular}{|l|l|l}
\hline DATE: November 1977 & VOLUMES:
\end{tabular}

PAGES: 80 REFERENCES: 115

ACQUISITION NO: 77-EHD-13 SOURCE: 16

KEY WORDS: Data Review Human Absorption Measurement Methods
Microwave

Public Health

Theory 
AUTHOR(S): Environmental Health Directorate

AUTHOR AFFILIATION: Minister of National Health and Welfare (Canada)

TITLE: Health Aspects of Radio Frequency and Microwave Radiation Exposure, Part 2

DATE: March 1978

VOLUMES :

PAGES : 115

REFERENCES : 313

ACQUISITION NO: 78-EHD-22

SOURCE: 16

\begin{tabular}{|lll} 
KEY WORDS: & $\begin{array}{l}\text { Behavior Effects } \\
\text { Data Review } \\
\text { Microwave }\end{array}$ & $\begin{array}{l}\text { Regulation } \\
\text { Research }\end{array}$ \\
& Standards
\end{tabular}

SUBJECT: BIOLOGIC EFFECTS

SPSPO INDEX NO: BE-45

$\operatorname{AUTHOR}(s)$ : Environmental Protection Agency

AUTHOR AFFILIATION: U. S. Environmental Protection Agency

TITLE: Environmental Assessment for the Satellite Power System - Concept Development and Evaluation Program - Microwave Health and Ecological Effects

\begin{tabular}{|c|l|l|l|}
\hline DATE: November 1980 & VOLUMES: & PAGES: 143 & REF \\
\hline ACQUISITION NO: DOE/ER/10035-2 & \\
\hline KEY WORDS: $\begin{array}{l}\text { Data Review } \\
\text { Exposure } \\
\text { Microwave }\end{array}$ & $\begin{array}{l}\text { Occupational Exposure } \\
\text { Public Health } \\
\text { Regulation }\end{array}$ \\
\hline
\end{tabular}


SUBJECT:, BIOLOGIC EFFECTS

AUTHOR (s): Formanek, V. C.

Gauger, J. R.

Emerle, E.

AUTHOR AFFILIATIION.: IIT Research Institute

TITLE: Susceptibility of Cardiac Pacemakers to Modulated ELF Magnetic Fields

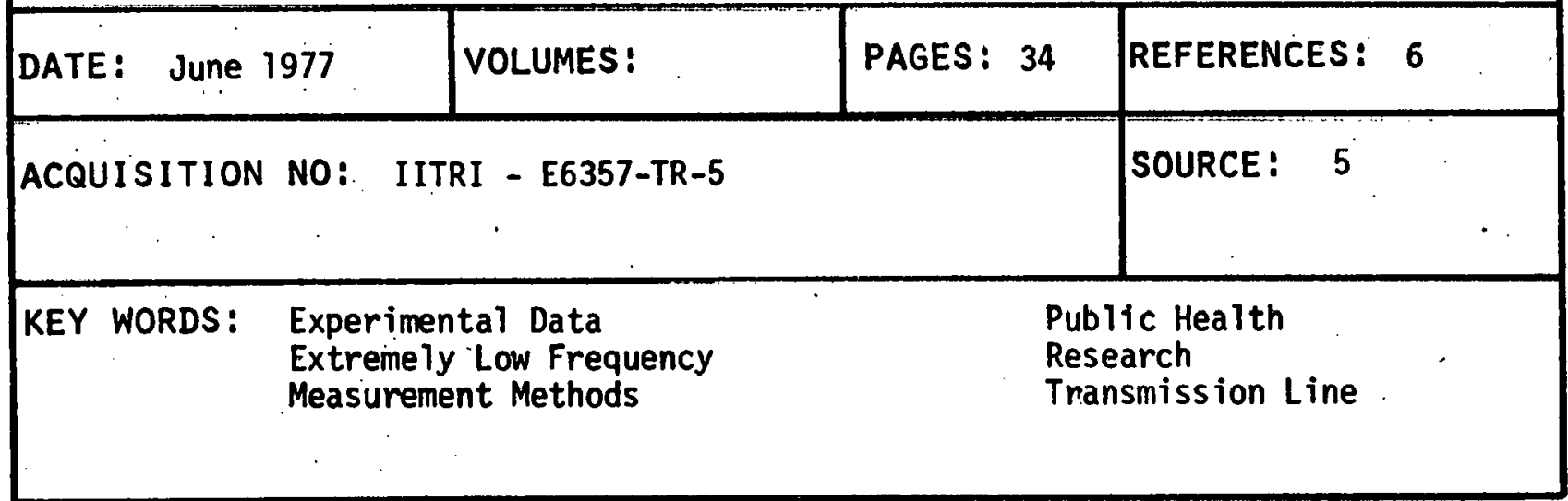

SUBJECT: BIOLOGIC EFFECTS

SPSPO INDEX NO: BE-10

$\operatorname{AUTHOR}(S)$ : Gandhi, 0. P.

AUTHOR AFFILIATION: University of Utah

TITLE: 2450 MHz Microwave Absorption in Large and Small Animals and its Biochemical Effects on Birds and Reptiles

\begin{tabular}{|c|c|c|c|c|c|}
\hline DATE : & February 1978 & VOLUMES: 1 & PAGES : 99 & REFERENCES: & \\
\hline \multicolumn{2}{|c|}{ ACQUISITION NO: } & $\begin{array}{l}\text { NAS2-9555 } \\
\text { UTEC ND 78-015 }\end{array}$ & & \multicolumn{2}{|l|}{ SOURCE : } \\
\hline
\end{tabular}


AUTHOR(S): Gordon, Z. V.

AUTHOR AFFILIATION: Israel Program for Scientific Translations, Ltd.

TITLE:- Biological Effect of Microwaves, in Occupational Hygiene (Translated from Russian!

\begin{tabular}{|c|c|c|c|c|}
\hline DATE : & & VOLUMES: & PAGES: 102 & $\begin{array}{l}\text { REFERENCES: } \\
\text { Bibliography }\end{array}$ \\
\hline ACQUISITION & NO: & $\begin{array}{l}\text { TT70-50087 } \\
\text { NASA TTF-633 }\end{array}$ & & SOURCE: 5 \\
\hline KEY WORDS: & $\begin{array}{l}\text { Data } \\
\text { Expo } \\
\text { Huma! }\end{array}$ & $\begin{array}{l}\text { Review } \\
\text { ure } \\
\text { Absorption }\end{array}$ & $\begin{array}{l}\text { Mammal Studies } \\
\text { Microwave } \\
\text { Occupational Expos }\end{array}$ & \\
\hline
\end{tabular}

\section{SUBJECT: BIOLOGIC EFFECTS}

AUTHOR(S): GouTd, J.L.

Kirschvink, J. L.

Deffeyes, K. S.

AUTHOR AFFILIATION: Princeton University

TITLE: Bees Have Magnetic Remanence

DATE: April 1978

VOLUMES:

\begin{tabular}{|l|l|}
\hline PAGES: 3 & REFERENCES: 20 \\
\hline & SOURCE: 2
\end{tabular}

ACQUISITION NO: 0036-8075/78/0915-1026

SOURCE : 2

KEY WORDS: Behavior Effects

Experimental Data

Insects

\section{Research}

Theory 
SUBJECT: BIOLOGIC EFFECTS

AUTHOR(s): Gould, James L.

AUTHOR AFFILIATION: Princeton University

TITLE: The Case for Magnetic Sensitivjty in Birds and Bees (Such as It Is)

DATE: May-June 1980

VOLUMES:

PAGES: 12

REFERENCES : 42

ACQUISITION NO: ISSN-0003-0996

SOURCE： 35

KEY WORDS: Avian Species

Behavior Effects

Experimental Data

Exposure

Research

Theory

SUBJECT: BIOLOGIC EFFECTS

AUTHOR (s): Guy, A. W.

Webb, M. D.

McDouga 11, J. A.

AUTHOR AFFILIATION: University of Washington

TITLE: R F Radiation Absorption Patterns: Human and Animal Model ing Data

DATE: September 1977

VOLUMES:

PAGES: 71

REFERENCES : 11

ACQUISITION NO: DHEW (NIOSH) 77-183

SOURCE: 20

KEY WORDS: : Data Review

Dos imetry

Human Absorption
Measurement Methods

Research

Specific Absorption 
AUTHOR (s): Ham, W. T. Sliney, D: H.

AUTHOR AFFILIATION: Electric Power Research Institute

TITLE: Physiological Effects of Redirected Solar Radiation

DATE: August 1978 VOLUMES:

PAGES: 56 REFERENCES: 27

ACQUISITION NO: EPRI ER-651 SOURCE : 26

KEY WORDS: Data Review Exposure Infrared
Public Health

Solar Effects

UI traviolet

SUBJECT: BIOLOGIC EFFECTS

AUTHOR(S): Hazzard, D. G.

AUTHOR AFFILIATION: U. S. Public Health Service

TITLE: Symposium on Biological Effects and Measurement of Radio Frequency/ Microwaves

\begin{tabular}{|c|c|c|c|c|}
\hline DATE : February & y 1977 & VOLUMES : & PAGES: 38 & REFERENCES : 282 \\
\hline ACQUISITION & NO: & $D A-77-8026$ & & SOURCE: 5 \\
\hline KEY WORDS : $\begin{array}{l}B \\
D \\
M\end{array}$ & \multicolumn{2}{|c|}{$\begin{array}{l}\text { Behavior Effects } \\
\text { Dosimetry } \\
\text { Mamial Studies }\end{array}$} & \multicolumn{2}{|c|}{$\begin{array}{l}\text { Measurement Methods } \\
\text { Occupational Exposure } \\
\text { Research. }\end{array}$} \\
\hline
\end{tabular}




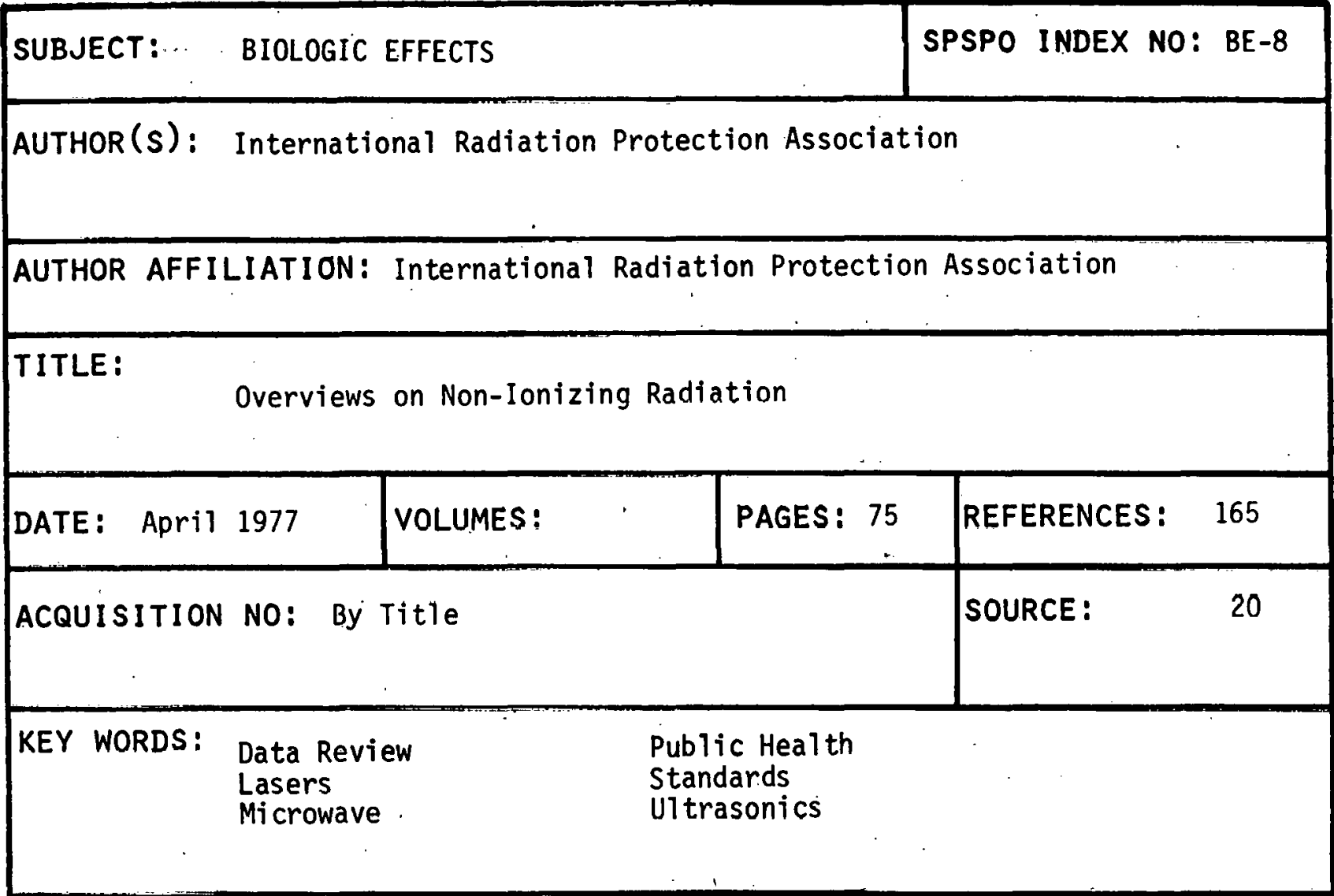

\section{SUBJECT: BIOLOGIC EFFECTS}

SPSPO INDEX NO: BE-37

AUTHOR (S): Ishimaru, A; (Ed)

AUTHOR AFFILIATION: The American Geophysical Union

TITLE: Biological Effects of EM Waves

\begin{tabular}{|c|c|c|c|c|}
\hline DATE: & $\begin{array}{l}\text { November - } \\
\text { December } 1979\end{array}$ & VOLUUMES : & PAGES: 354 & REFERENCES: 70 \\
\hline
\end{tabular}

\begin{tabular}{|lll|}
\hline ACQUISITION NO: & RASCAD 14 (6S) \\
& ISSN 0048-6604 & SOURCE \\
\hline KEY WORDS: & $\begin{array}{l}\text { Behavior Effects } \\
\text { Blood-Brain Barrier } \\
\text { Extremely Low Frequency }\end{array}$ & $\begin{array}{l}\text { Mammal Studies } \\
\text { Measurement Methods } \\
\text { Microwave }\end{array}$
\end{tabular}




\section{AUTHOR (s): (1) Johnson; C. C. (Ed.) \\ (2) Shore, M. L. (Ed.)}

AUTHOR AFFILIATION: (1) University of Utah

(2) Bureau of Radiological Health

TITLE: : Biological Effects of Electromagnetic Waves

\begin{tabular}{|l|l|l|l|}
\hline DATE: October 1975 & VOLUMES: 2 & PAGES: 955 & REFERENCES: 1089 \\
\hline ACQUISITION NO: $\begin{array}{l}\text { FDA-77-8010 (Vol. 1) } \\
\text { FAD-77-8011 (Vol. 2) }\end{array}$ & SOURCE: 5 \\
\hline KEY WORDS: & $\begin{array}{l}\text { Behavior Effects } \\
\text { Mosimetry } \\
\text { Mammal Studies }\end{array}$ & $\begin{array}{l}\text { Measurement Methods } \\
\text { Microwave } \\
\text { Research }\end{array}$ \\
\hline
\end{tabular}

\begin{tabular}{|l|l|}
\hline SUBJECT: BIOLOGIC EFFECTS & SPSPO INDEX NO: BE-48 \\
\hline
\end{tabular}

AUTHOR(S): Johnson Space Center

AUTHOR AFFILIATION: National Aeronautics and Space Administration

TITLE: Assessment of Zero Gravity Effects on Space Worker Health and Safety

\begin{tabular}{|l|l|l|l|}
\hline DATE: November 1980 & VOLUMES: & PAGES: 122 & REFERENCES: 63 \\
\hline ACQUISITION NO: DOE/ER/10025-TI & & SOURCE: 5 \\
\hline KEY WORDS: & $\begin{array}{l}\text { Data Review } \\
\text { Mitigating Strategy } \\
\text { Occupationa1. Exposure }\end{array}$ & $\begin{array}{l}\text { Space Flight } \\
\text { Space Workers } \\
\text { Zero Gravity }\end{array}$ \\
\hline
\end{tabular}




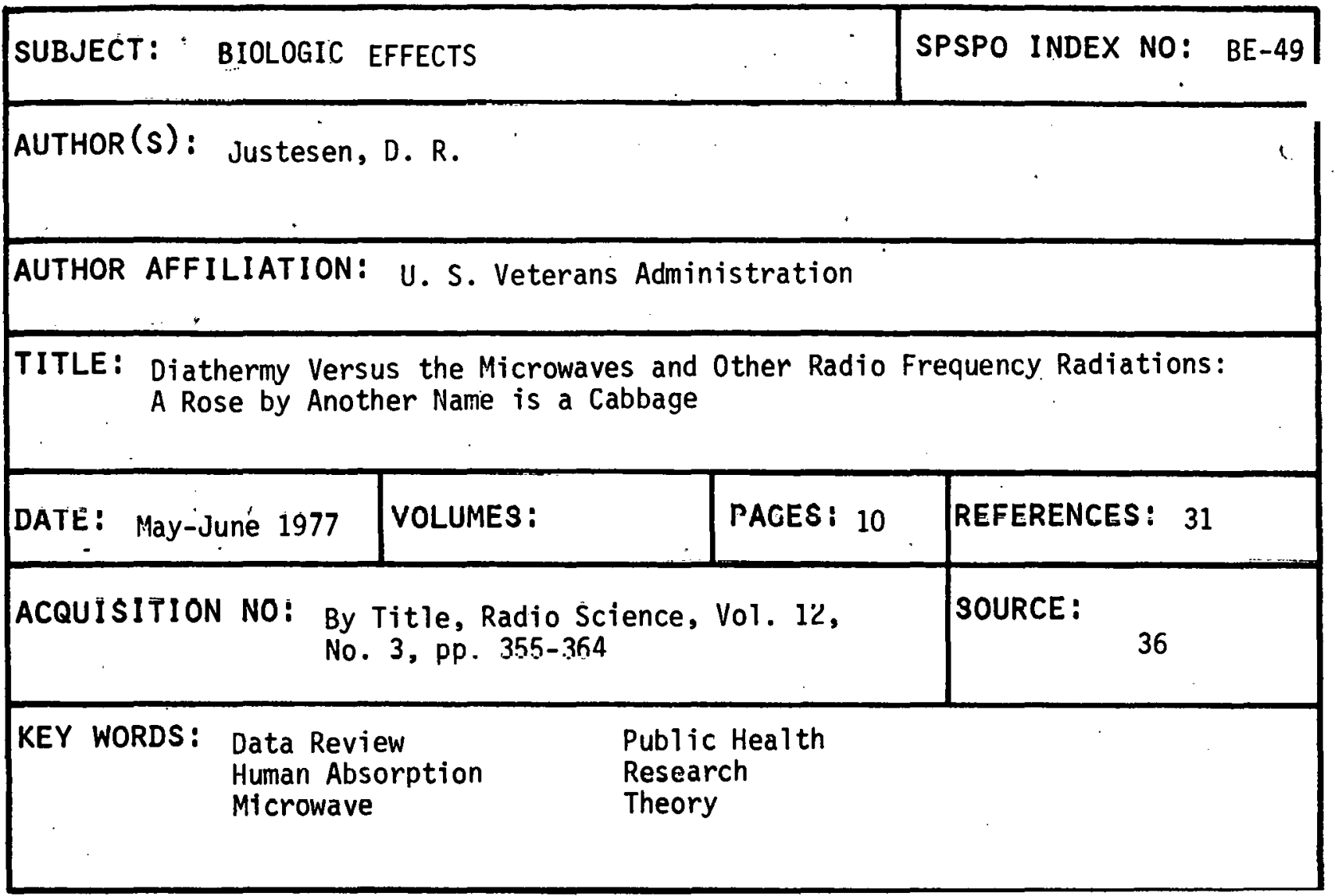

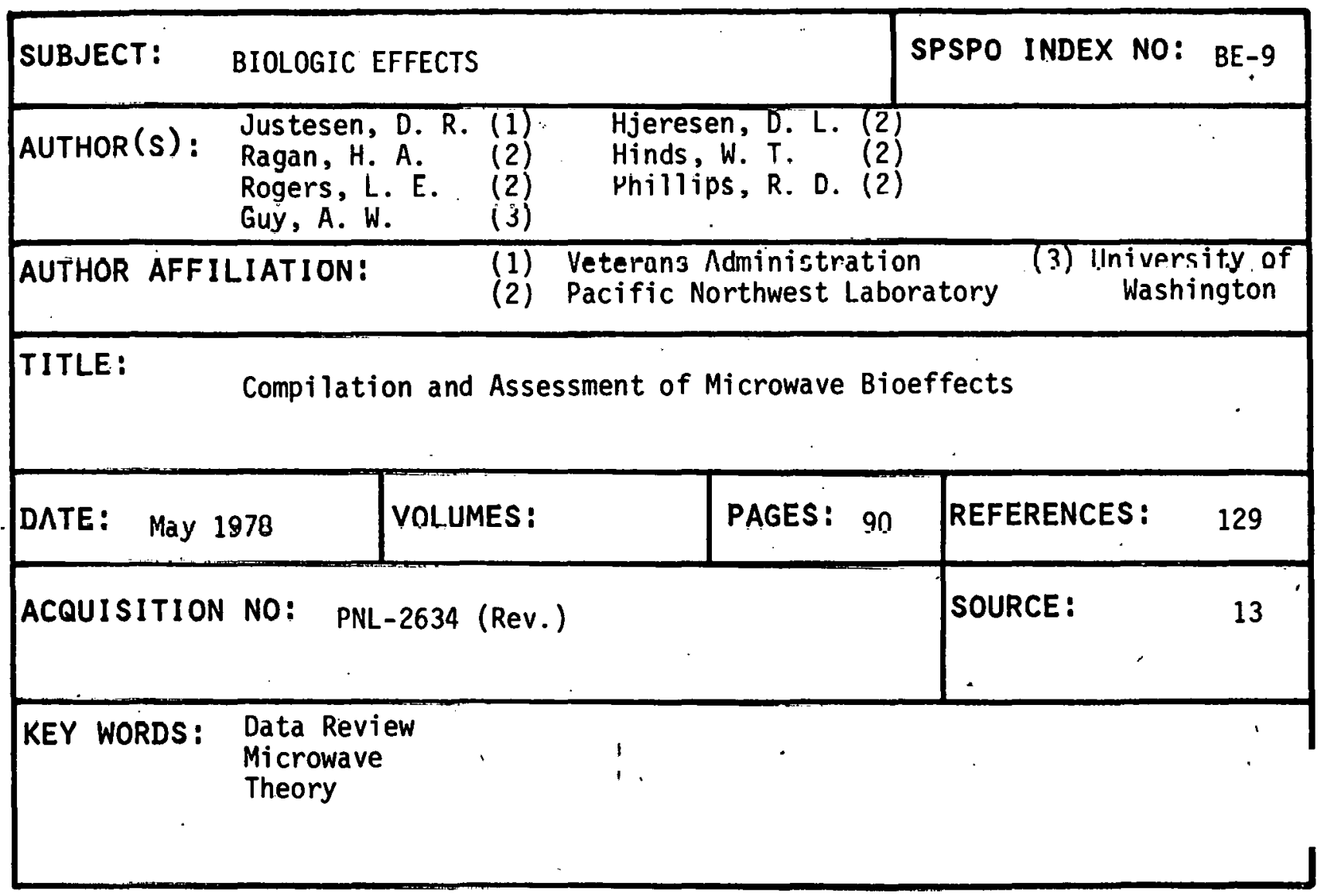


AUTHOR(S): Lawrence Berkeley Laboratory

AUTHOR AFFILIATION: Lawrence Berkeley Laboratory

TITLE: Ionizing Radiation Risks to Satellite Power Systems (SPS) Workers in Space

DATE: December 1980

VOLUMES:

\begin{tabular}{l|l} 
PAGES: 58 & REFERENCES : 58
\end{tabular}

ACQUISITION NO: DOE/ER-0094

SOURCE: 5

KEY WORDS: Charged Particles

Cosmic Rays

Dose Levels

Health Risks

Radiation Exposure

Shielding

SUBJECT: BIOLOGIC EFFECTS

SPSPO INDEX NO: BE-19

AUThOR(s): Mallalieu, R. C.

AUTHOR AFFILIATION: The Johns Hopkins University

TITLE: A Model of the Microwave Intensity Distribution with in the US Embassy in Moscow, 1966 to 1977

\begin{tabular}{|l|l|l|l|}
\hline DATE: August 1980 & VOLUMES: & PAGES: 81 & REFERENCES: 53 \\
\hline ACQUISITION NO: FS-80-166 & SOURCE: 5 \\
\hline KEY WORDS: & $\begin{array}{l}\text { Dos imetry } \\
\text { Exposure } \\
\text { Human Absorption }\end{array}$ & $\begin{array}{l}\text { Microwave } \\
\text { Occupational Exposure } \\
\text { Public Health }\end{array}$ \\
\hline
\end{tabular}




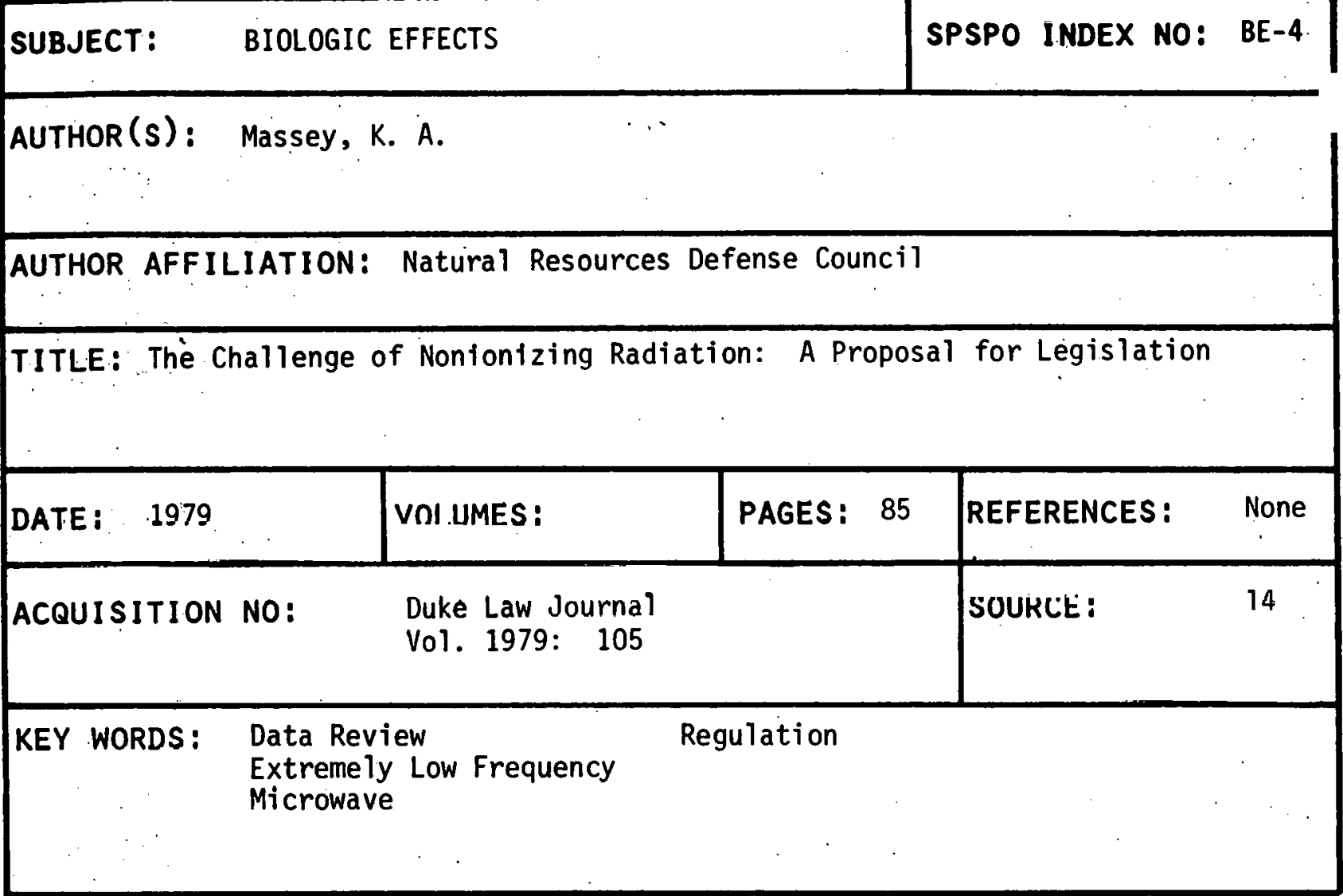

\begin{tabular}{|l|l|}
\hline SUBJECT: BIOLOGIC EFFECTS & SPSPO INDEX NO: BE-35 \\
\hline AUTHOR(S): Michaelson, S. M. &
\end{tabular}

\section{AUTHOR AFFILIATION: University of Rochester}

TITLE: Analysis of Studies Related to Blologic Effects and Health Implications of Exposure to Power Frequencies

\begin{tabular}{|l|l|l|l|}
\hline DATE: 1979 & VOLUMES: & PAGES: 16 & REFERENCES: 43 \\
\hline ACQUISITION NO: $0191-5398 / 79 / 030217-16$ & SOURCE: 33 \\
\hline KEY WORDS: & $\begin{array}{l}\text { Data Review } \\
\text { Extremely Low Frequency } \\
\text { Measurement Methods }\end{array}$ & $\begin{array}{l}\text { Research } \\
\text { Standards } \\
\text { Theory }\end{array}$ \\
\hline
\end{tabular}


AUTHOR(s): National Research Council

AUTHOR AFFILIATION: National Academy of Sciences

TITLE: Analysis of the Exposure Levels and Potential Biologic Effects of the PAVE PAWS Radar Syster:

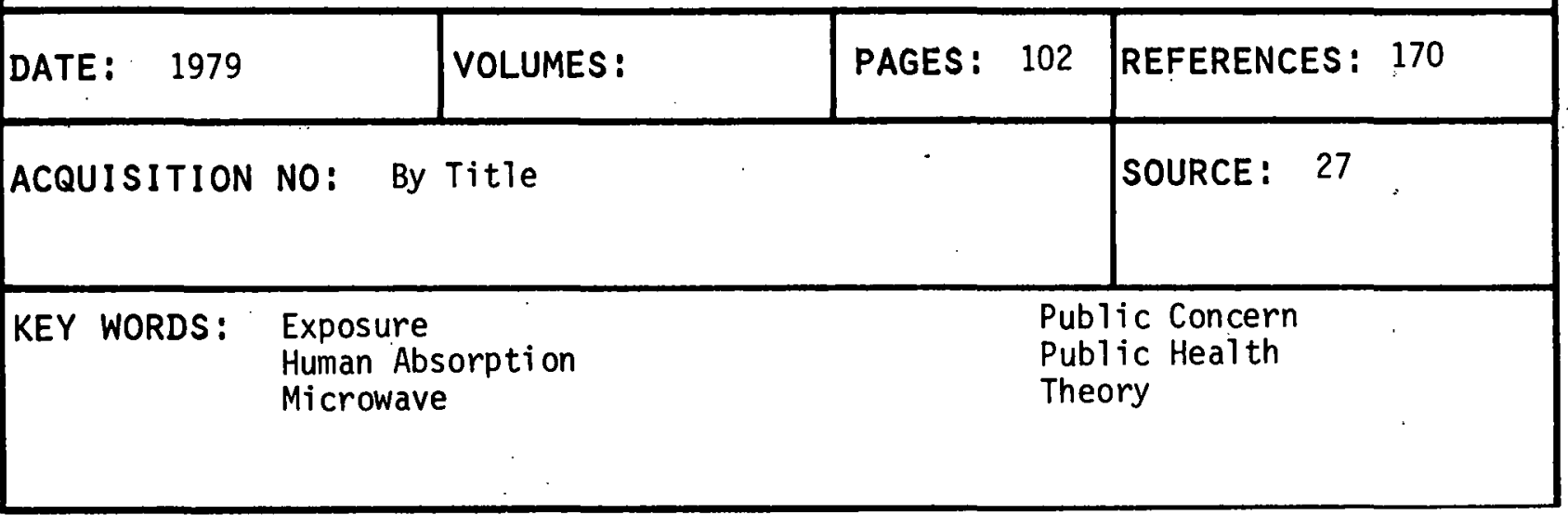

SUBJECT: BIOLOGIC EFFECTS

SPSPO INDEX NO: BE-30

AUTHOR(s): National Telecommunications and Information Administration

AUTHOR AFFILIATION: U.S. Department of Commerce

TITLE: Fifth Report on Program for Control of Electromagnetic Pollution of the Environment: The Assessment of Biological Hazards of Nonionizing Electromagnetic Radiation.

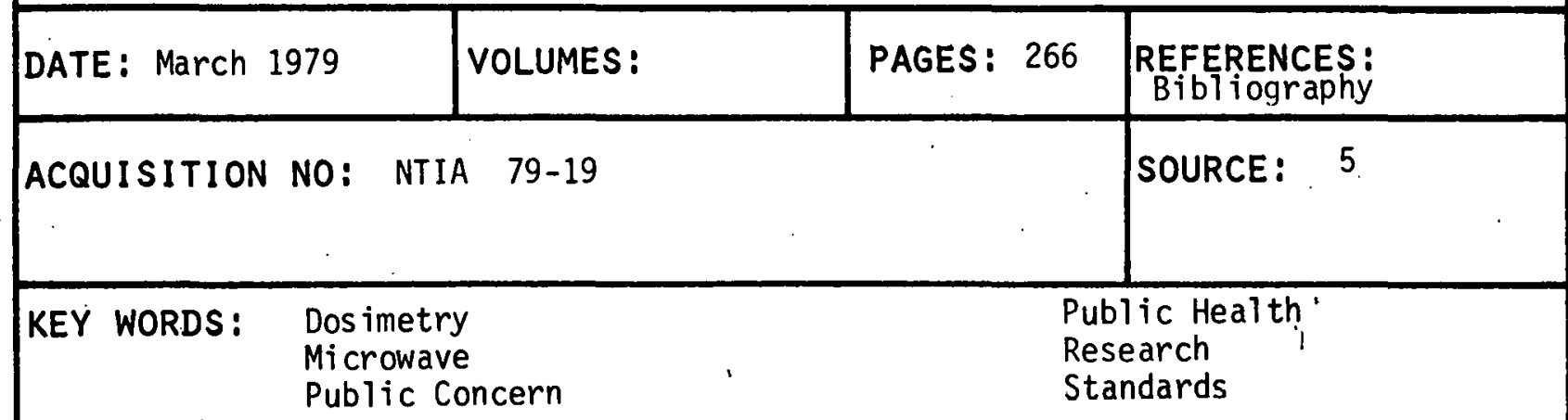




$\begin{array}{lll}\text { AUTHOR }(s): ~ & \text { Ozerski, P. } \\ & \text { Ostrowski,k. } & \text { Silverman, C. } \\ & \text { Shore, M. L. } & \text { Suess, M. J. } \\ & \text { Waldeskog, B. }\end{array}$

AUTHOR AFFILIATION: World Health Organization

Minister, Health and

U. S. Dept. Health, Education and Welfare

Social Welfare (Poland)

TITLE: Biologic Effects and Health Hazards of Microwave Radiation

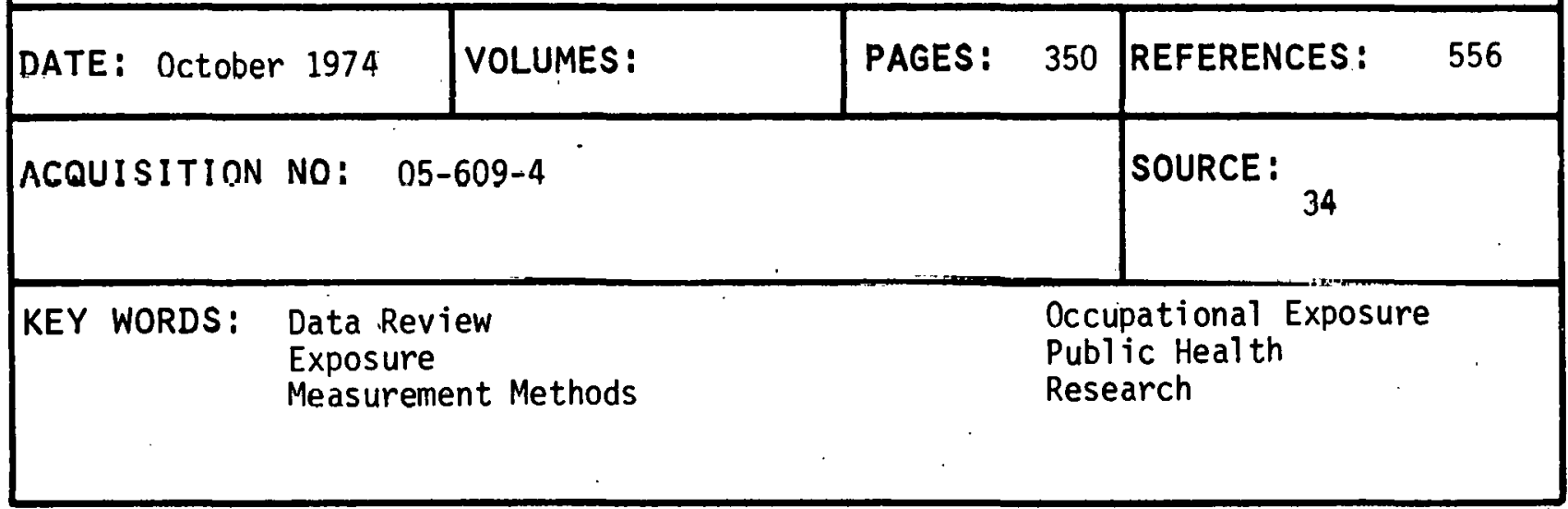

\begin{tabular}{|c|c|c|c|c|c|}
\hline SUBJECT: & \multicolumn{3}{|c|}{ BIOLOGIC EFFECTS } & \multicolumn{2}{|c|}{ SPSPO INDEX NO: BE-53 } \\
\hline $\operatorname{AUTHOR}(s):$ & \multicolumn{5}{|c|}{$\begin{array}{l}\text { Poon, P. T. Y. } \\
\text { Baker, } i . \dot{R} . \\
\text { Kuo, T. j. }\end{array}$} \\
\hline \multicolumn{6}{|c|}{ AUTHOR AFFILIATION: Jet Propulsion Laboratory } \\
\hline TITLE: & \multicolumn{5}{|c|}{$\begin{array}{l}\text { Preliminary Examination of Satellite Power System (SPS) } \\
\text { Occupational Health Impacts }\end{array}$} \\
\hline DATE: Octobe & October 1978 & VOLUMES: & PAGES: 40 & REFERENCES : & 12 \\
\hline ACQUISITION & NO: & \multicolumn{2}{|c|}{$900 \overline{-}-820$ Rev. A. } & SOURCE : & 4 \\
\hline KEY WORDS: & \multicolumn{5}{|c|}{$\begin{array}{l}\text { Health Risks } \\
\text { Ionizing Radiation } \\
\text { Radiation Exposure }\end{array}$} \\
\hline
\end{tabular}




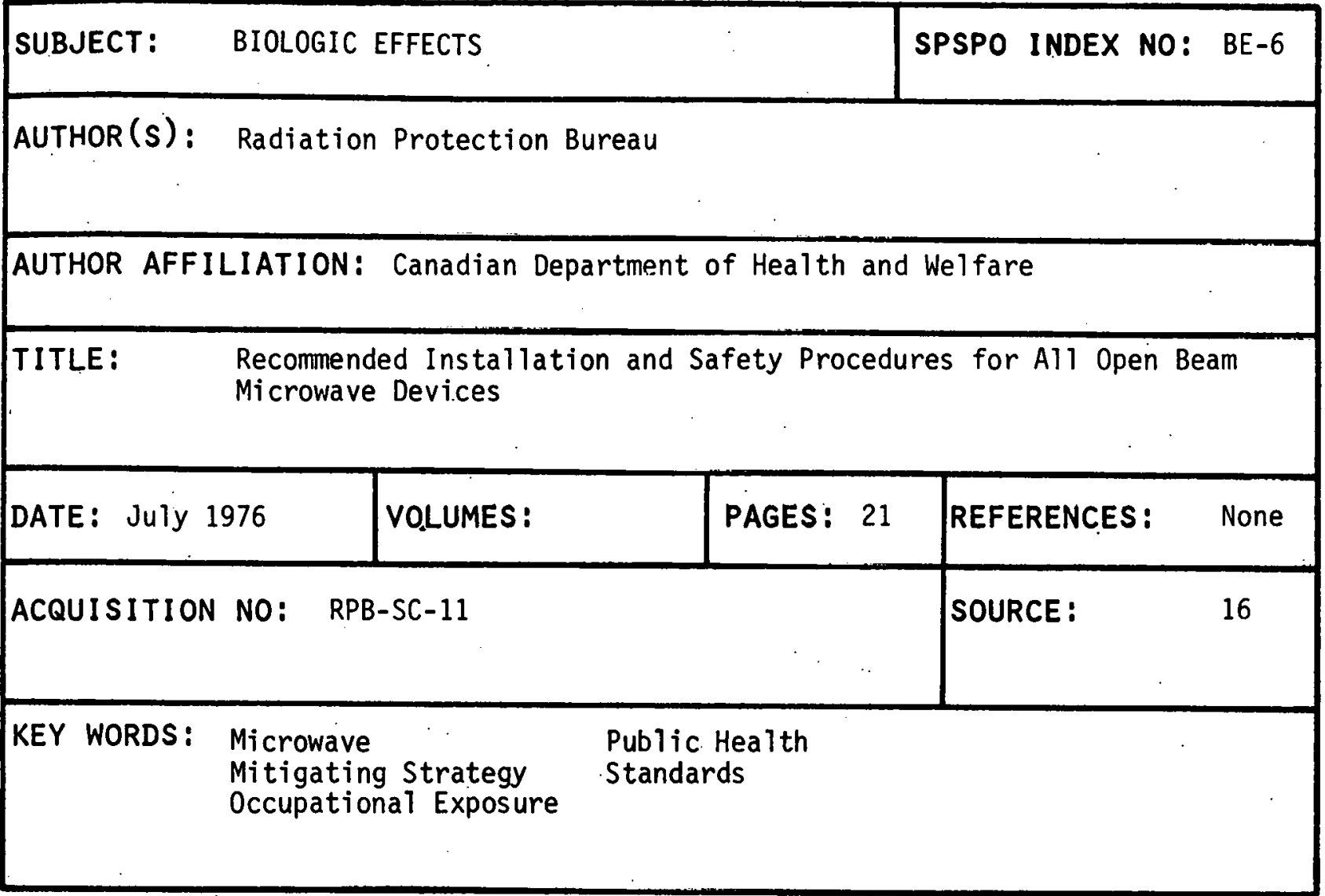

\begin{tabular}{|l|l|}
\hline SUBJECT: BIOLOGIC EFFECTS & SPSPO INDEX NO: BE-47 \\
\hline
\end{tabular}

$\operatorname{AUTHOR}(S)$ : Repacholi, M. H.

AUTHOR AFFILIATION: Department of Health and Welfare (Canada)

TITLE: Proposed Exposure Limits for Microwave and Radiofrequency Radiations in Canada

DATE: 1978 VOLUMES: PAGES: 13 REFERENCES: 51

ACQUISITION NO: By Title; Journal of Microwave SOURCE: 39 Power, $13(2)$

KEY WORDS: Data Review Microwave Occupational Exposure
Regulation

Specific Absorbtion Standards 


\section{SUBJECT: $\quad$ BIOLOGIC EFFECTS}

AUTHOR (s): Rish, W. R.

Morgan, M. G.

AUTHOR AFFILIATION: Carnegie-Mellon University

TITLE: Regulating Possible Health Effects from AC Transmission Line Electromagnetic Fields

\begin{tabular}{|l|l|l|lc|}
\hline DATE: OCtober 1979 & VOLUMES: & PAGES: .12 & REFERENCES: & 47 \\
\hline ACQUISITI ON NO: & $0018-9219 / 79 / 1000-1416$ & SOURCE : & 6 \\
& & & \\
\hline
\end{tabular}

KEY WORDS: Data Review

Exposure

Extremely Low Frequency

Mitigating Strategy

Regulation

Transmisston Line

SUBJECT: BIOLOGIC EFFECTS

SPSPO INDEX NO: BE-36

$\operatorname{AUTHOR}(s)$ : (1) Rogers, L. E.

(1) Gilbert, R. 0.

(2) Lee, J. M.

(¿) Bracken, T. D.

AUTHOR AFFILIATION: (1) Battelle Pacific Northwest Laboratories

(2) Bonneville Power Administration

TITLE: BPA $1100 \mathrm{kV}$ Transmission System Development-Environment Studies

\begin{tabular}{|l|l|l|l|}
\hline DATE: February 1979 & VOLUMES: & PAGES: 9 & REFERENCES: 20 \\
\hline ACQUISITION NO: 1979 IEEE Winter PES & SOURCE: 6 \\
\hline KEY WORDS: Avian Species & & \\
1 & $\begin{array}{l}\text { Mamperimental Data Studies } \\
\text { Extremely Low Frequency }\end{array}$ & $\begin{array}{l}\text { Transmission Line } \\
\text { Vegetation Effects }\end{array}$ \\
\hline
\end{tabular}




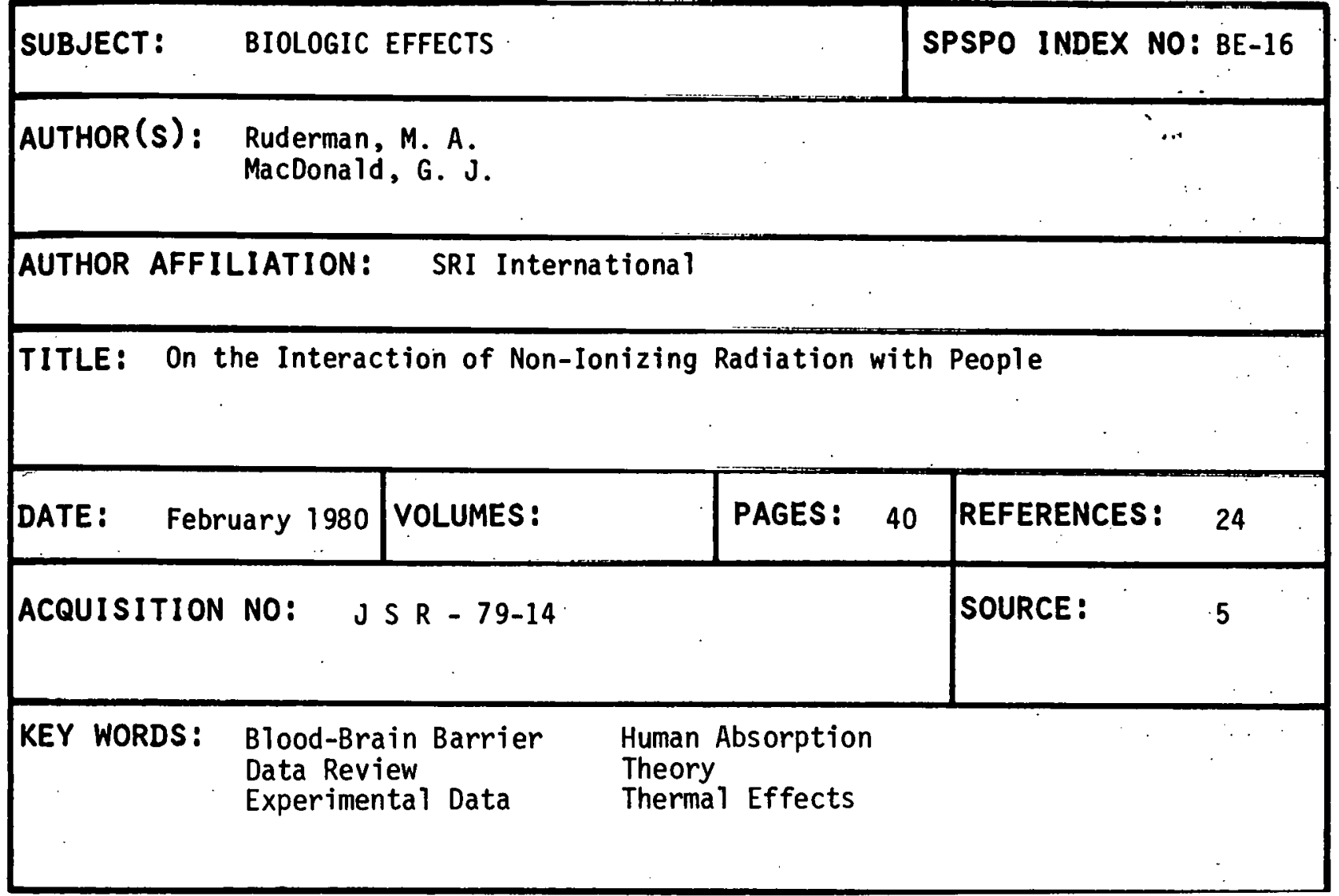

\begin{tabular}{|c|c|c|c|c|}
\hline \multicolumn{4}{|c|}{ BIOLOGIC EFFECTS } & SPSPO INDEX NO: BE-5 \\
\hline \multicolumn{5}{|c|}{ AUTHOR(s): Schiefelbein, S. } \\
\hline \multicolumn{5}{|c|}{ AUTHOR AFFILIATION: Saturday, Review Magazine } \\
\hline \multicolumn{5}{|c|}{ TITLE: $\quad$ The Invisible Threat } \\
\hline DATE: Sept & ember 1979 & VOLUMES: & PAGES : 5 & REFERENCES : \\
\hline \multicolumn{4}{|c|}{$\begin{array}{ll}\text { ACQUISITION NO: } & \text { Saturday Review } \\
& \text { Vol. } 6 \text {, No. } 18 \\
& \text { pp. } 16-20\end{array}$} & SOURCE : 15 \\
\hline \multicolumn{5}{|c|}{$\begin{aligned} \text { KEY WORDS: } & \text { Data Review } \\
& \text { Exposure } \\
& \text { Extremely Low. Frequency }\end{aligned}$} \\
\hline
\end{tabular}




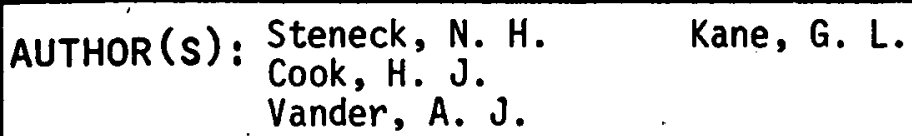

AUTHOR AFFILIATION: University of Michigan

TITLE: The Oriains of U. S. Safety Standards for Microwave Radiation

DATE: June 13,1980

VOLUMES :

\begin{tabular}{ll|l} 
PAGES: 8 & REFERENCES: 54
\end{tabular}

ACQUISITION NO: Science - Vol. 208. No. No. 4449

SOURCE: 7

KEY WORDS: Exposure

Microwave

Occupational Exposure
Public Concern

Public Health

Regulation

SUBJECT: BIOLOGIC EFFECTS

SPSPO INDEX NO: $B E-26$

$\operatorname{AUTHOR}(s):$ Valentino, A. R.

AUTHOR AFFILIATION: IIT Research Institute

TITLE: A Tutorial: Non-Ionizing Electromagnetic Fields-Biological Effects Terminology

DATE: 1979

VOLUMES:

\begin{tabular}{ll|l} 
PAGES: 4 & REFERENCES: 5
\end{tabular}

ACQUISITION NO: 0191-5398/79/020155-04

SOURCE: 33

KEY WORDS: Dosimetry

Exposure

Extremely Low Frequency

Microwave

Regulation

Standards 
AUTHOR(s): Watts, J. W.

Wright, J. J.

AUTHOR AFFILIATION: NASA Marshall Space Flight Center

TITLE: Charged Particle Radiation Environment for the Spacelab and Dther Misstions in Low Earth Orbit

\begin{tabular}{|c|c|c|c|c|c|}
\hline DATE: June & 1975 & VOLUMES: & PAGES: 68 & REFERENCES : & 17 \\
\hline ACQUISITION & NO: & \multicolumn{2}{|c|}{ IASA T:M X-64936 } & SOURCE : & 4 \\
\hline KEY WORDS : & \multicolumn{2}{|c|}{$\begin{array}{l}\text { Cosmic Rays } \\
\text { Dose Levels } \\
\text { Electron Flux }\end{array}$} & $\begin{array}{l}\text { Proton Flux } \\
\text { Solar Flares } \\
\text { Spacelab }\end{array}$ & & \\
\hline
\end{tabular}

\section{SUBJECT: BIOLOGIC EFFECTS}

$\operatorname{AUTHOR}(s)$ : White, M.

AUTHOR AFFILIATION: Lawrence Berkeley Laboratory

TITLE: Effects on Biological Systems of Reflected Light from the Satellite Power System

\begin{tabular}{|c|c|c|c|c|}
\hline DATE: March & 1981 & VOLUMES: & PAGES: 54 & REFERENCES : 19 \\
\hline \multicolumn{4}{|c|}{ ACQUISITION NO: Not Available } & SOURCE: 5 \\
\hline KEY WORDS: & $\begin{array}{l}\text { Data } \\
\text { Eye } \\
\text { Growt }\end{array}$ & $\begin{array}{l}\text { iew. } \\
\text { ds Developmer }\end{array}$ & & $\begin{array}{l}\text { nal Studies } \\
\text { lected Light } \\
\text { etation Effects }\end{array}$ \\
\hline
\end{tabular}


$\operatorname{AUTHOR}(s)$ : Yamaura, I.

AUTHOR AFFILIATION: Electrotechnical Laboratory (Japan)

TITLE: Measurements of $1.8-2.6 \mathrm{GHz}$ Microwave Attenuation in the Human Torso

DATE: August 1977 VOLUMES: PAGES: 4 REFERENCES: 8 \begin{tabular}{l|l|l}
- & & \\
\hline
\end{tabular}

ACQUISITION NO: - MTT-25, No. 8 SOURCE:

KEY WORDS: Experimental Data Human Absorption Measurement Methods 


\section{COMPARATIVE ASSESSMENT \\ (CATEGORY CA)}

Documents in this category include information which is useful for comparing various energy options with the SPS concept. Existing energy options are included as are emerging energy technologies. Some documents characterize technologies, while others only provide information on certain aspects of a technology (such as performance or related environmental effects, for example). Methods which have been developed for comparing options also are included.

KEY WORDS:

Applications

Atmospheric Effects

Central Solar

Climate Effects

Economics

Electric Distribution

Energy Conversion

Environmental Effects

Fossil Sys tems

Health Effects

Heliostats

Land Use

Line Collectors

Me thodology

Models

Net Energy

Nuclear Systems

Photovoltaic

Plate Collectors

Power Tower

Resources

Safety

Societal Effects

Solar. Energy

Solar Performance

Storage
PAGE NUMBER:

$54,57,58,60,64$

63,68

$53,54,55,56,57,58,60,62,67$

63

$54,55,56,57,58,59,60,61,62,64,65$, $66,67,68$

60

$56,61,66$

$55,59,63,64,66,67,68$

$53,56,59,62,63,64,66,67,68$

$53,55,59,62,63,66,67$

66

57,64

65

$54,55,57,58,60,61,62,63,64$

$54,57,58,60,61$

$56,59,60,62,65$

$53,56,59,61,62,63,64,66,67,68$

$54,56,57,58,59,63,64$,

65

66

$55,58,59,61,66,67$

53,62

67

$53,54,55,56,57,60,65,68$

$54,55,56,65,66,67$

57,61 
(Comparative Assessment

Category CA continued)

KEY WORDS :

Terrestrial solar

Waste Heat
PAGE NUMBER:

$53,54,55,56,57,58,59,62,64,65,66,67$

63

RELATED CATEGORIES:

Energy Alternatives and Policy (EA)

A1l other categories include information on SPS 
SUBJECT: COMPARATIVE ASSESSMENT

AUTHOR(s): Argonne National Laboratory

AUTHOR AFFILIATION: Argonne National Laboratory

TITLE: Comparative Health and Safety Assessment of the Satellite Power System and Other Electrical Generation Alternatives

\begin{tabular}{|c|c|c|c|c|}
\hline DATE: & aber 1980 & VOLUMES: & PAGES: 154 & REFERENCES: 133 \\
\hline \multicolumn{3}{|c|}{ ACQUISITION NO: DOE/ER-0091 } & & SOURCE: 5 \\
\hline KEY WORDS: & $\begin{array}{l}\text { Central S } \\
\text { Fossil Sy } \\
\text { Health Ef }\end{array}$ & $\begin{array}{l}\text { Solar } \\
\text { ystems } \\
\text { ffects }\end{array}$ & \multicolumn{2}{|c|}{$\begin{array}{l}\text { Nuclear Systems } \\
\text { Safety } \\
\text { Terrestrial Solar }\end{array}$} \\
\hline
\end{tabular}

SUBJECT: COMPARATIVE ASSESSMENT

SPSPO INDEX NO: CA-31

AUTHOR(S): Argonne National Laboratory

AUTHOR AFFILIATION: Argonne National Laboratory

TITLE: NOT AVAILABLE - Final Comparative Assessment of SPS and Six other Energy Technologies expected to be published in March 2981

\begin{tabular}{|c|c|c|c|}
\hline DATE: & VOLUMES: & PAGES : & REFERENCES : \\
\hline ACQUISITION NO: & & & SOURCE: 5 \\
\hline
\end{tabular}

KEY WORDS : 

$\operatorname{AUTHOR}(\mathrm{s})$ : (1) Ayres, R. U.
(2) Ridker, R. G.
(4) Arnold, J.
(3) Watson, W. D.
(1) Tayi, G.

AUTHOR AFFILIATION: (1) Carnegie-Mellon University

(2) Resources for the Future, Inc.

(3) U.S. Geological Survey

(4) Variflex Corporation

TITLE: Alternative Future Scenarios for the SPS Comparative Assessment

DATE: August 1980

VOLUMES :

PAGES : 151

REFERENCES : 58

ACQUISITIION NO: ANL/EES-TM-120

SOURCE： 5

KEY WORDS: Applications

Mode 1s

Economics

Methodology

\section{SUBJECT : \\ $\operatorname{AUTHOR}(s)$ : \\ Bel1, C. R.}

COMPARATIVE ASSESSMENT

SPSPO INDEX NO: CA-5

AUTHOR AFFILIATION: Jet Propulsion Laboratory $\begin{array}{ll}\text { TITLE:. } & \text { Assessment of a Solar Photovoltaic Power Conversion System for } \\ \text { Central Station Application }\end{array}$

\begin{tabular}{|c|l|l|l|}
\hline DATE: June 1975 & VOLUMES: & PAGES: 57. & REFERENCES: 22 \\
\hline ACQUISITION NO: $\quad \begin{array}{l}900-702 \\
\text { EM } 342294\end{array}$ & SOURCE: \\
\hline KEY WORDS: $\begin{array}{l}\text { Central Solar } \\
\text { Photovoltaic } \\
\text { Solar Energy }\end{array}$ & $\begin{array}{l}\text { Solar Performance } \\
\text { Terrestrial Solar }\end{array}$ \\
\hline
\end{tabular}




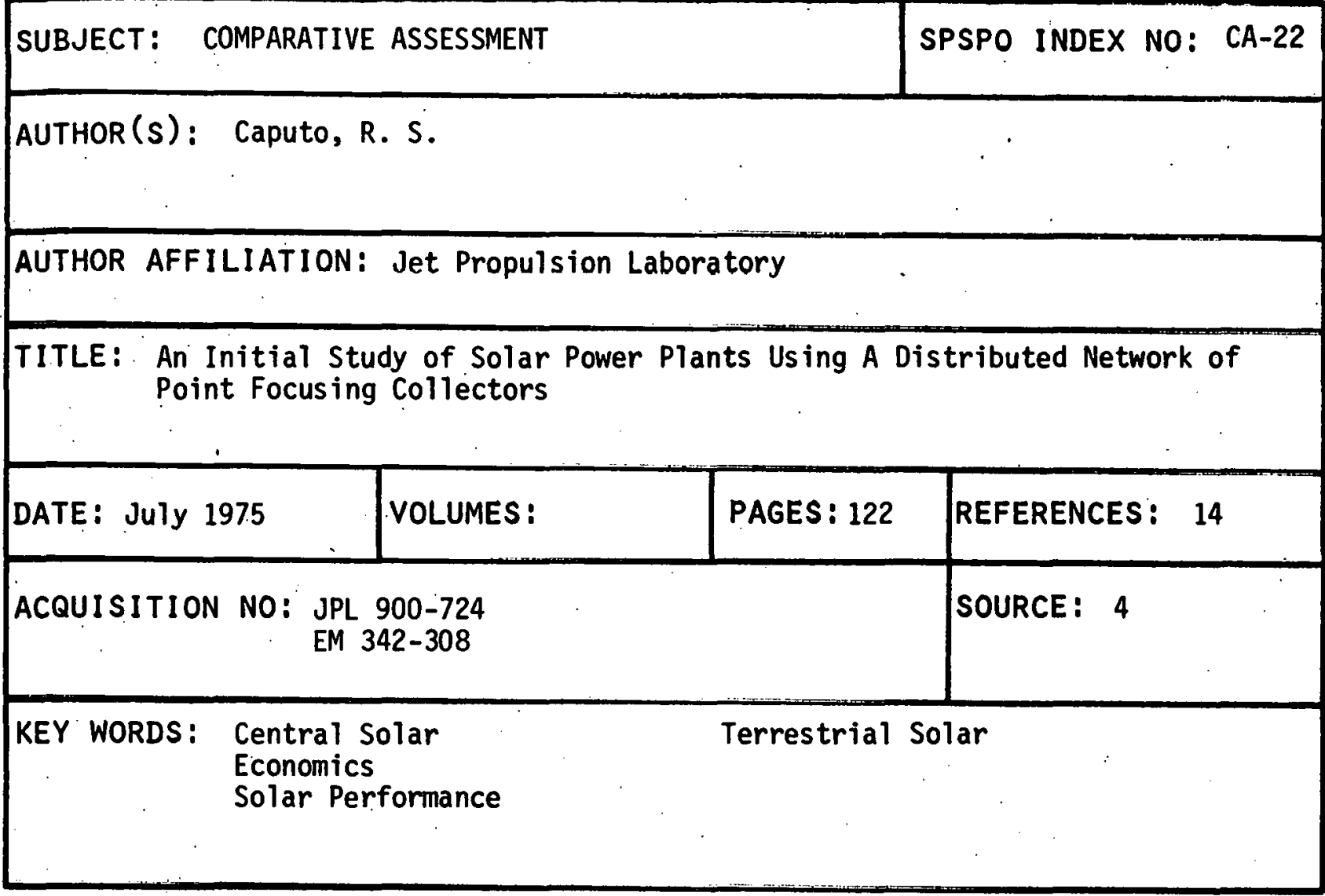

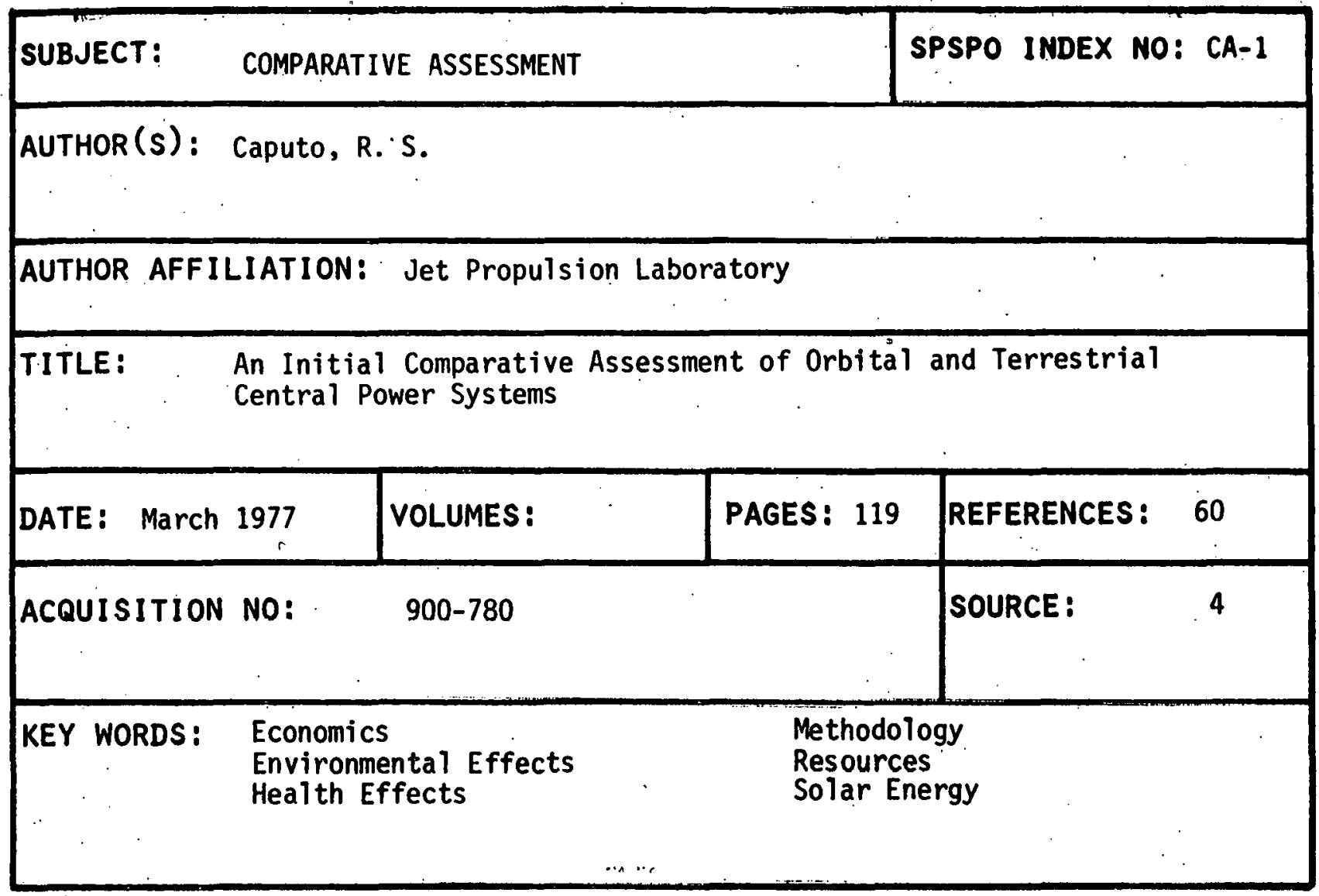




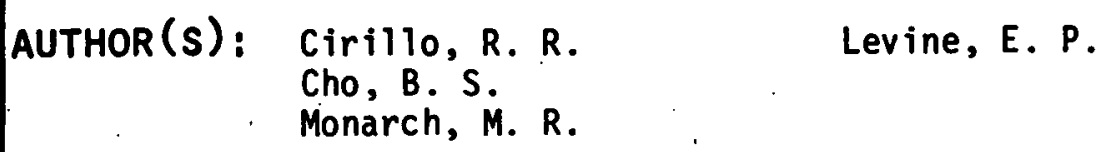

\begin{tabular}{|c|c|c|c|c|c|c|}
\hline DATE: & 11980 & VOLUMES: & PAGES: & 136 & REFERENCES: & 74 \\
\hline ACQUISITION & NO: & DOE/ER-0056 & & & SOURCE: & 5 \\
\hline KEY WORDS : & $\begin{array}{l}\text { Energy } \\
\text { Fossil } \\
\text { Net En }\end{array}$ & $\begin{array}{l}\text { Conversion } \\
\text { Systems } \\
\text { ergy }\end{array}$ & & \multicolumn{3}{|c|}{$\begin{array}{l}\text { Nuclear Systems } \\
\text { Photovoltaic } \\
\text { Terrestrial Solar }\end{array}$} \\
\hline
\end{tabular}

SUBJECT: COMPARATIVE ASSESSMENT

AUTHOR (S): Claverie, M. J.

Dupas, A. P.

AUTHOR AFFILIATION: Centre National de la Recherche Scientifique

TITLE: Preliminary Evaluation of Ground and Space Solar Electricity Mark.ot in 2025

\begin{tabular}{|c|c|c|c|c|}
\hline DATE: October & 1978 & VOLUMES: & PAGES: 19 & REFERENCES: 8 \\
\hline \multicolumn{4}{|c|}{ ACQUISITION NO: } & 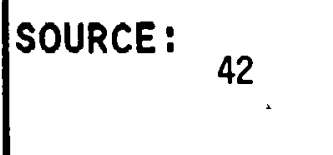 \\
\hline KEY WORDS: & \multicolumn{2}{|c|}{$\begin{array}{l}\text { Central Solar } \\
\text { Economics } \\
\text { Photovoltaic }\end{array}$} & \multicolumn{2}{|c|}{$\begin{array}{l}\text { Solar Energy } \\
\text { Solar Performance } \\
\text { Terrestrial Sölar }\end{array}$} \\
\hline
\end{tabular}


AUTHOR(S): Claverie, M. J.

Dupas, A. P.

AUTHOR AFFILIATION: Centre National de la Recherche Scientifique

TITLE: Terrestrial Versus Space Baseload Solar Electricity

\begin{tabular}{|c|c|c|c|c|}
\hline DATE: Septembe & er 1979 & VOLUMES: & PAGES: 22 & REFERENCES : 18 \\
\hline ACQUISITION N & No: If & F 79-172 & & $\begin{array}{l}\text { SOURCE : } \\
\\
\end{array}$ \\
\hline KEY WORDS : & $\begin{array}{l}\text { Central } \\
\text { Economic } \\
\text { Land Use }\end{array}$ & $\begin{array}{l}\text { Solar } \\
\text { s }\end{array}$ & \multicolumn{2}{|c|}{$\begin{array}{l}\text { Photovoltaics } \\
\text { Storage } \\
\text { Terrestrial Solar }\end{array}$} \\
\hline
\end{tabular}

\begin{tabular}{|c|c|c|c|c|c|}
\hline SUBJECT: & \multicolumn{3}{|c|}{ COMPARATIVE ASSESSMENT } & \multicolumn{2}{|c|}{ SPSPO INDEX NO: CA-8 } \\
\hline $\operatorname{AUTHOR}(s):$ & \multicolumn{5}{|c|}{ Coates, V. T. } \\
\hline \multicolumn{6}{|c|}{ AUTHOR AFFILIATION: George Washington Untversity } \\
\hline TITLE: & \multicolumn{5}{|c|}{ A Handbook of Technology Assessment } \\
\hline DATE: March & 1978 & VOLUMES: & PAGES : 102 & REFERENCES : & 25 \\
\hline ACQUISITION & NO: & \multicolumn{2}{|l|}{ TID-28503 } & SOURCE: & 5 \\
\hline KEY WORDS : & \multicolumn{3}{|c|}{$\begin{array}{l}\text { Applications } \\
\text { Economics } \\
\text { Methodology }\end{array}$} & \multicolumn{2}{|l|}{$\begin{array}{l}\text { Models } \\
\text { Solar Energy }\end{array}$} \\
\hline
\end{tabular}


AUTHOR (S): Crowley, J. H.

Ziegler, E. J:

AUTHOR AFFILIATION: Argonne National Laboratory

TITLE: Satellite Power. Systems (SPS) Cost Review

\begin{tabular}{|l|l|l|l}
\hline DATE: May 1980 & VOLUMES: & PAGES: 88 & REFERENCES: 26 \\
\hline
\end{tabular}

ACQUISITION NO: DOE/TIC-11190

SOURCE: 5

KEY WORDS: Economics

Photovoltaic

Resources

\begin{tabular}{|l|l|}
\hline SUBJECT: COMPARATIVE ASSESSMENT & SPSPO INDEX NO: CA-23 \\
\hline AUTHOR(S): Doane, J. W. \\
O'Toole, R. P.
\end{tabular}


AUTHOR(S): Drucker, H.

AUTHOR AFFILIATION: Pacific Northwest Laboratory

TITLE: Pacific Northwest Laboratory Annual Report for 1979 to the DOE Assistant Secretary for Environment; Part 1, Biomedical Sciences

\begin{tabular}{|c|c|c|c|c|}
\hline \multicolumn{2}{|c|}{ DATE: February 1980} & VOLUMES: & PAGES: 338 & $\begin{array}{l}\text { REFERENCES: } \\
\text { Bibliography }\end{array}$ \\
\hline \multicolumn{4}{|c|}{ ACQUISITIION NO: PNL $-3300^{\circ}$} & SOURCE: 5 \\
\hline KEY WORDS : & $\begin{array}{l}\text { Enviror } \\
\text { Fossil } \\
\text { Health }\end{array}$ & $\begin{array}{l}\text { ntal Effects } \\
\text { stems } \\
\text { fects }\end{array}$ & & ear Systems \\
\hline
\end{tabular}

SUBJECT: . COMPARATIVE ASSESSMENT

AUTHOR(s): Energy Research and Development Administration

AUTHOR AFFILIATION: Energy Research and Development Administration

TITLE: Solar Energy in America's Future

\begin{tabular}{|c|c|c|c|c|}
\hline DATE: March & 1977 & VOLUMES: & PAGES: 127 & $\begin{array}{l}\text { REFERENCES: } \\
\text { Bibliography }\end{array}$ \\
\hline ACQUISITION & NO: & DSE-115/1 & & SOURCE: 5 \\
\hline KEY WORDS : & \multicolumn{2}{|c|}{$\begin{array}{l}\text { Economics } \\
\text { Environmental Effects } \\
\text { Net Energy }\end{array}$} & \multicolumn{2}{|c|}{$\begin{array}{l}\text { Photovoltaics } \\
\text { Resources. } \\
\text { Terrestrial Solar }\end{array}$} \\
\hline
\end{tabular}




$$
\begin{aligned}
& \text { AUTHOR (S): Fraley, D: W. } \\
& \text { McDonald, C. L } \\
& \text { Carter, N. E. }
\end{aligned}
$$

\begin{tabular}{|c|c|c|c|c|c|}
\hline DATE: & 1978 & VOLUMES: & PAGES: 48 & REFERENCES : & 21 \\
\hline \multicolumn{4}{|c|}{ ACQUISITION NO: PNL-SA-6619 } & \multicolumn{2}{|l|}{ SOURCE: 13} \\
\hline \multicolumn{3}{|c|}{$\begin{aligned} \text { KEY WORDS: } & \begin{array}{l}\text { Applications } \\
\text { Economics } \\
\text { Methodology }\end{array}\end{aligned}$} & \multicolumn{2}{|c|}{$\begin{array}{l}\text { Models } \\
\text { Net Energy } \\
\text { Solar Energy }\end{array}$} & \\
\hline
\end{tabular}

\section{AUTHOR AFFILIATION: Pacific Northwest Laboratory.}

TITLE: A Review of Issues and Applications of Net Energy Analysis

SUBJECT: COMPARATIVE ASSESSMENT

$\operatorname{AUTHOR}(s)$ : Fujita, T.

AUTHOR AFFILIATION: Jet Propulsion Laboratory

\begin{tabular}{|c|c|c|c|}
\hline DATE: March 1976 & VOLLUMES : & PAGES: 124 & REFERENCES: 46 \\
\hline \multicolumn{3}{|c|}{$\begin{array}{ll}\text { ACQUISITION NO: JPL } 900-734 \\
\text { EM } 342-332\end{array}$} & SOURCE : 4 \\
\hline \multicolumn{2}{|c|}{$\begin{aligned} \text { KEY WORDS: } & \text { Central Solar } \\
& \text { Economics } \\
& \text { Electric Distribution }\end{aligned}$} & \multicolumn{2}{|l|}{$\begin{array}{l}\text { Methodology } \\
\text { Models }\end{array}$} \\
\hline
\end{tabular}

TITLE: Preliminary Studies of Electrical and Hydrogen Pipeline Distribution and Cost Optimization of Electrical Transmission Lines 
AUTHOR(s): Fujita, T.

AUTHOR AFFILIATION: Jet Propulsion Laboratory

TITLE: Preliminary Data on Electrical Energy Storage Systems Utility Applications

\begin{tabular}{|l|l|l|l|}
\hline DATE: Apri1 1976 & VOLUMES: & PAGES: 48 & REFERENCES: 9 \\
\hline ACQUISITION NO: JPL $900-738$ & & SOURCE : 4 \\
\hline
\end{tabular}

KEY WORDS: Economics

Energy Conversion

Storage Systems

SUBJECT: COMPARATIVE ASSESSMENT

SPSPO INDEX NO: $\mathrm{CA}-19$

$\begin{aligned} & \text { AUTHOR (S): } \text { Gallagher, J. M. } \\ & \text { Carasso, i... }\end{aligned}$

Barany, $R$.

Zimmermann, R. G.

AUTHOR AFFILIATION: Bechtel Corporation

TITLE: Direct Requirements of Capital, Manpower, Materials and Equipment for Selected Energy Futures

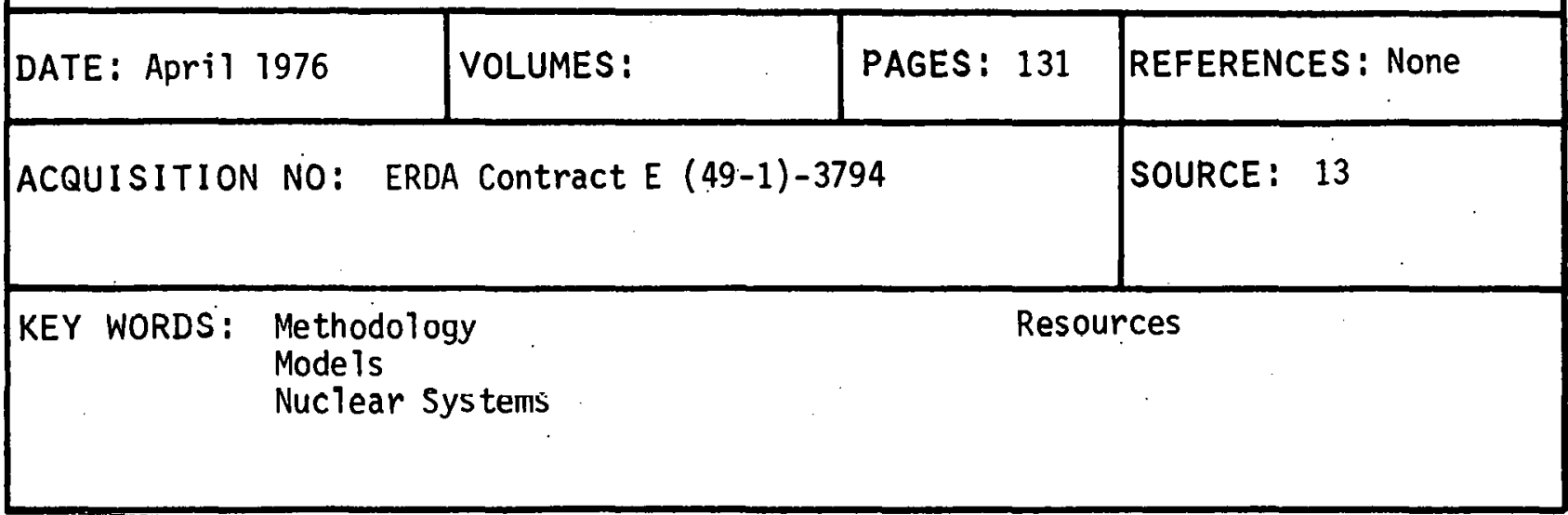




\begin{tabular}{|c|c|c|c|c|c|c|}
\hline \multirow{2}{*}{$\begin{array}{l}\text { SUBJECT: } \\
\text { AUTHOR (S): }\end{array}$} & \multicolumn{2}{|c|}{ COMPARATIVE ASSESSMENT } & 1 & \multicolumn{3}{|c|}{ SPSPO INDEX NO: CA-11 } \\
\hline & \multicolumn{6}{|c|}{$\begin{array}{l}\text { Habegger, L. J. } \\
\text { Gasper, J.R. } \\
\text { Brown, C. D. }\end{array}$} \\
\hline \multicolumn{7}{|c|}{ AUTHOR AFFILIATION: Argonne National Laboratory } \\
\hline \multicolumn{7}{|c|}{$\begin{array}{l}\text { TITLE: Health and Safety: Prel iminary Comparative Assessment of the } \\
\text { Satellite Power System (SPS) and Other Energy Alternatives }\end{array}$} \\
\hline DATE: & 11980 & VOLUMES: & PAGES: & 123 & REFERENCES : & 69 \\
\hline \multicolumn{2}{|c|}{ ACQUISITION NO: } & OE/ER-0053 & & & SOURCE : & 5 \\
\hline \multicolumn{3}{|c|}{$\begin{array}{ll}\text { KEY WORDS: } & \text { Central Solar } \\
& \text { Fossii systems } \\
& \text { Health Effects }\end{array}$} & & \multicolumn{2}{|c|}{$\begin{array}{l}\text { Nuclear Sys tems } \\
\text { Safety } \\
\text { Terrestrial Solar }\end{array}$} & \\
\hline
\end{tabular}

SUBJECT: COMPARATIVE ASSESSMENT

SPSPO INDEX NO: CA-20

AUTHOR (s): Energy Analysis, Institute for

AUTHOR AFFILIATION: Oak Ridge Associated Universities

TITLE: Net Energy Analys is of Five Energy Systems

DATE: April 1977 VOLUMES:

PAGES: 55 REFERENCES: 11

ACQUISITION NO: ORAU/IEA (R) -77-12

SOURCE： 13

KEY WORDS: Economics

Fossil Systems

Methodology 
$\operatorname{AUTHOR}(s)$ : Kellermeyer, D. A.

AUTHOR AFFILIATION: Argonne National Laboratory

TITLE: Climate and Energy: A Comparative Assessment of the Satellite Power System (SPS) and Aiternative Energy Technologies

\begin{tabular}{|c|c|c|c|c|c|}
\hline DATE: Januar & y 1980 & VOLUMES: & PAGES : & 73 & REFERENCES: 88 \\
\hline \multicolumn{3}{|c|}{ ACQUISITION NO: DOE/ER-0050 } & & & SOURCE : 5 \\
\hline KEY WORDS: & $\begin{array}{l}\text { Atmosph } \\
\text { Climate } \\
\text { Fossil }\end{array}$ & $\begin{array}{l}\text { ic Effects } \\
\text { ffects } \\
\text { stems }\end{array}$ & \multicolumn{3}{|c|}{$\begin{array}{l}\text { Nuclear Systems } \\
\text { Photovoltaic } \\
\text { Waste Heat }\end{array}$} \\
\hline
\end{tabular}

SUBJECT: COMPARATIVE ASSESSMENT

AUTHOR(S): Levine, E.P.

Senew, M. J.

Cirillo, R. R.

AUTHOR AFFILIATION: Argonne National Laboratory

TITLE: Comparative Assessment of Environmental Welfare Issues Associated with the Satellite Power System (SPS) and Alternative Technologies

DATE: Apri1 1980

VOLUMES:

PAGES: 87 : REFERENCES : 53

ACQUISITION NO: DOE/ER-0055

SOURCE: 5

KEY WORDS: Environmental Effects

Fossil Systems

Health Effects

Methodology

Nuclear Systems

Photovoltaic 


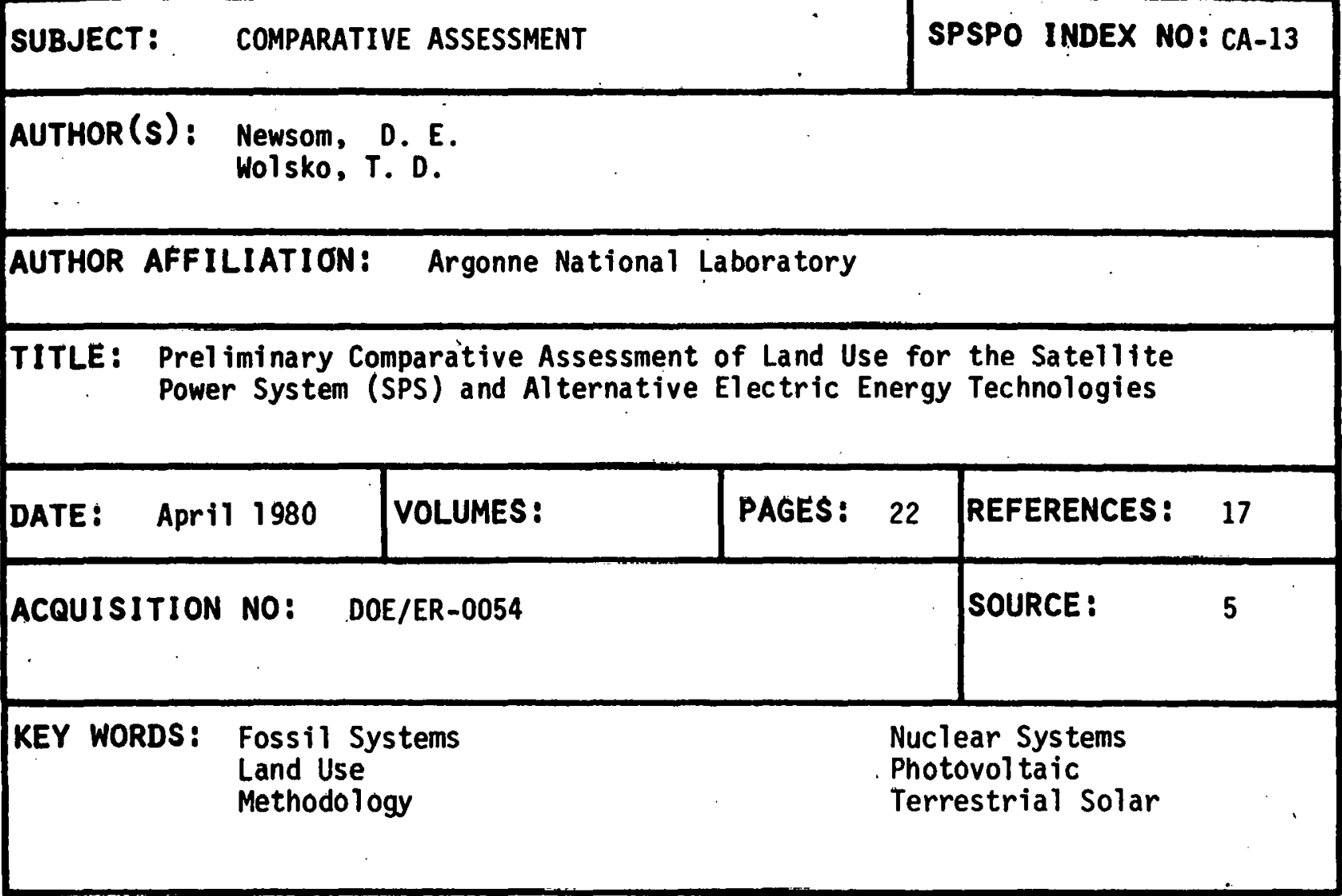

\section{SUBJECT: COMPARATIVE ASSESSMENT}

AUTHOR(s): Samsa, M. E.

AUTHOR AFFILIATION: Argonne National Laboratory

TITLE: Selection of Alternative Central-Station Technologies for the Satellite Power System (SPS) Comparative Assessment

DATE: Apri1 1980

VOLUMES:

PAGES: 15

REFERENCES: None

ACQUISITION NO: . DOE/ER-0052

SOURCE: 5

KEY WORDS: $\begin{aligned} \text { Applications } \\ \text { Economics } \\ \text { Environmental Effects }\end{aligned}$

Fossil Systems

Nuclear Sys tems

Photovoltaic 
AUTHOR(S): Selcuk, M. K.

AUTHOR AFFILIATION:

Jet Propulsion Laboratory

TITLE: $\quad$ Prel iminary. Assessment of Flat Plate Collector Solar Thermal Power Plants

\begin{tabular}{|c|l|l|l|}
\hline DATE: March 1975 & VOLUMES: & PAGES: 104 & REFERENCES: \\
\hline ACQUISITION NO: $\quad \begin{array}{l}900-692 \\
\text { EM 342-283 }\end{array}$ & SOURCE: & 4 \\
\hline KEY WORDS: & $\begin{array}{l}\text { Economics } \\
\text { Net Energy } \\
\text { Plate Collectors }\end{array}$ & $\begin{array}{l}\text { Solar Energy } \\
\text { Terrestrial Solar }\end{array}$ \\
\hline
\end{tabular}

\begin{tabular}{|c|c|c|c|c|c|c|}
\hline SUBJECT: & \multicolumn{4}{|c|}{ COMPARATIVE ASSESSMENT. } & \multicolumn{2}{|c|}{ SPSPO INDEX NO: CA-7 } \\
\hline $\operatorname{AUTHOR}(s):$ & \multicolumn{6}{|c|}{ Selcuk, M. K. } \\
\hline \multicolumn{7}{|c|}{ AUTHOR AFFILIATION: Jet Propulsion Laboratory } \\
\hline TITLE : & \multicolumn{6}{|c|}{$\begin{array}{l}\text { A Preliminary Technical and Economic Assessment of Solar Power Plants } \\
\text { Using Line Concentrators }\end{array}$} \\
\hline DATE : & July 1975 & VOLUMES: & PAGES: & & REFERENCES: & 34 \\
\hline ACQUISITION & NO: & \multicolumn{3}{|l|}{$\begin{array}{l}900-704 \\
\text { EM } 342-302\end{array}$} & SOURCE: & 4 \\
\hline KEY WORDS: & \multicolumn{2}{|c|}{$\begin{array}{l}\text { Economics } \\
\text { Line Collectors } \\
\text { Net Energy }\end{array}$} & \multicolumn{4}{|c|}{$\begin{array}{l}\text { Solar Energy } \\
\text { Solar Performance } \\
\text { Terrestrial Solar }\end{array}$} \\
\hline
\end{tabular}




\section{SUBJECT :}

AUTHOR(s): Selcuk, M. K.

AUTHOR AFFILIATION: Jet Propulsion Laboratory

TITLE: Survey of Several Central Receiver Solar Thermal Power Plant Design Concepts

\begin{tabular}{|l|l|l|l}
\hline DATE: August 1975 & VOLUMES: & PAGES: 82 & REFERENCES: \\
\hline ACQUISITION NO: $\quad \begin{array}{l}900=714 \\
\text { EM } 342-311\end{array}$ & SOURCE: \\
\hline KEY WORDS: & $\begin{array}{l}\text { Economics } \\
\text { Energy Conversion } \\
\text { Heliostats }\end{array}$ & $\begin{array}{l}\text { Power Tower } \\
\text { Solar Performance } \\
\text { Terrestrial Solar }\end{array}$ \\
\hline
\end{tabular}

SUBJECT: COMPARATIVE ASSESSMENT

AUTHOR(S): Smith, K. R.

Weyant, $J$.

Hüldren, J. P.

AUTHOR AFFILIATION: Jet Propulsion Laboratory

TITLE: Evaluation of Conventional Power Systems

\begin{tabular}{|c|c|c|c|c|}
\hline DATE: July & 1975 & VOLUMES: & PAGES : 187 & REFERENCES : 236 \\
\hline \multicolumn{4}{|c|}{ ACQUISITION NO: ERG-75-5 } & SOURCE: 4 \\
\hline KEY WORDS: & \multicolumn{2}{|c|}{$\begin{array}{l}\text { Economics } \\
\text { Environmental Effects } \\
\text { Fossil Systems }\end{array}$} & & $\begin{array}{l}\text { Heal th Effects } \\
\text { Nuclear Systems } \\
\text { Resources. }\end{array}$ \\
\hline
\end{tabular}


AUTHOR(S): TRW Energy Systems Planning Division

AUTHOR AFFILIATION: TRW, InC.

TITLE: Characterization of Alternative Electric Generation Technologies for the SPS Comparative Assessment

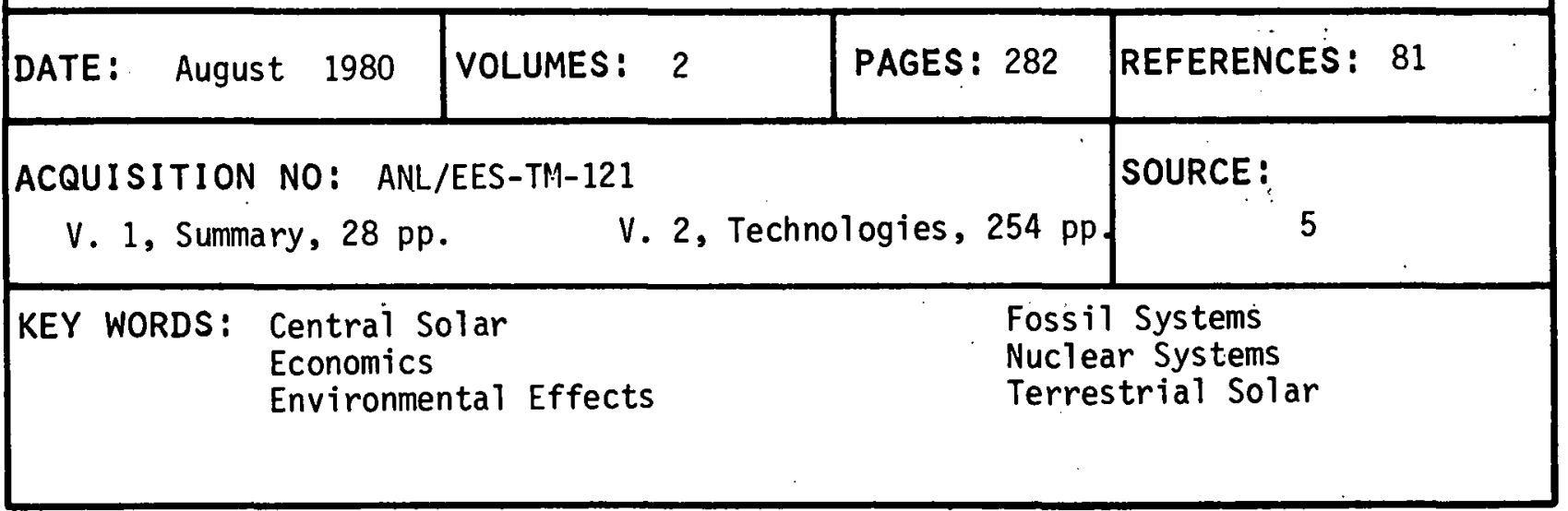

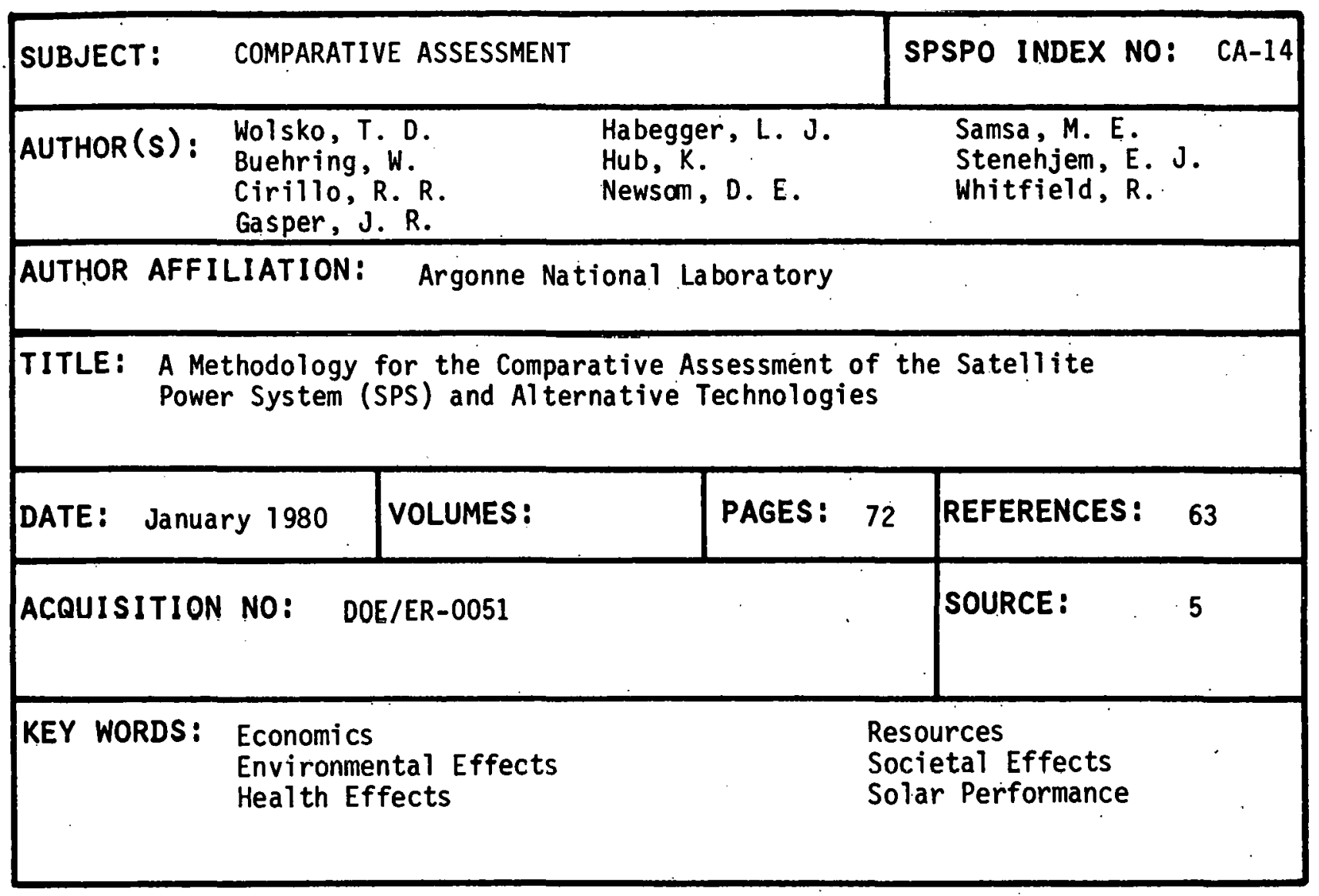




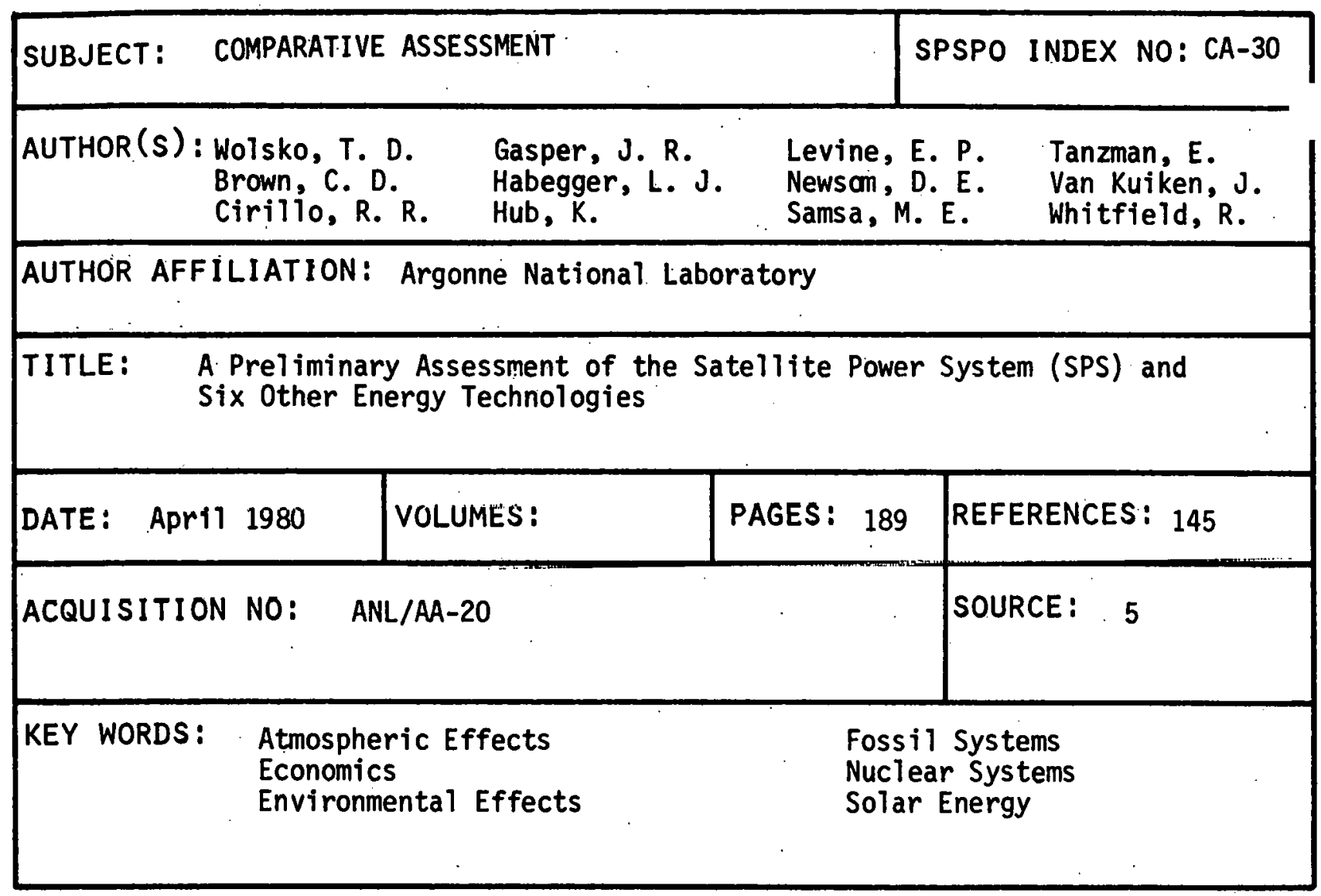

68 


\section{ENERGY ALTERNATIVES AND POLICY (CATEGORY EA)}

This category is a collection of reports and papers that deals with (1) SPS as a future energy alternative, (2) the role of SPS in national and/or international energy policy matters, or (3) other major energy technologies of interest to the development of SPS and their relationships with energy alternatives and policy.

KEY WORDS:

Comparative Methods

Concepts

Economics

Energy Options

Energy Ratio

Environmental Effects

Feasibility

Fossil Fuels

Geotherma 1

Hydroelectric

Inexhaustible Resources

International Interest

Issues

Legislation

Net Energy Analysis

Nuclear Power

Ocean Conversion

Policy Analys is

Political Process

Recommendations

Risks

Social Issues

Solar Conversion

Solar Power

Technology Requirements

Utility Decisions
PAGE NUMBER:

76

$71,73,75,77,78$

$72,73,75,76$

$71,72,73,74,75,77,78$

72,74

73

71,77

$71,73,78$

71

71

72,73

75

77

77

72,74

$71,73,77$

76

$72,73,75,77$

71

71

71

72

76

$71,73,75,76,77,78$

$72,76,77,78$

76 
(Energy Alternatives and Policy Category EA continued)

KEY WORDS:

PAGE NUMBER:

Wind Conversion

76

RELATED CATEGORIES:

Comparative Analysis (CA)

Social Issues (SI)

All other categorles include information on SPS 
SUBJECT: ENERGY ALTERNATIVES AND POLICY

SPSPO INDEX NO: EA-1

AUTHOR(s): American Institute of Aeronautics and Astronautics

AUTHOR AFFILIATION: American Institute of Aeronautics and Astronautics

TITLE: Solar Power Satellites

DATE: January 1979

VOLUMES:

\begin{tabular}{l|l} 
PAGES: 4 & REFERENCES: None
\end{tabular}

ACQUISITION NO: Astronautics and Aeronautics January 197.9

pp. 14-17

KEY WORDS: Concepts

Energy Options

Feasibility
Recommendations

Risks.

Technology Requirements

SUBJECT: ENERGY ALTERNATIVES AND POLICY

SPSPO INDEX NO: EA-9

$\begin{aligned} \text { AUTHOR (S): } & \begin{array}{l}\text { Babcock, W. H. } \\ \text { Siegel ; S. B. } \\ \text { Swanson, C. A. }\end{array}\end{aligned}$

AUTHOR AFFILIATION:

PRC Energy Analys is Company

TITLE:

Energy Demands, $1972-2000$

\begin{tabular}{|c|c|l|l|}
\hline DATE: April 1979 & VOLUMES: & PAGES: 150 & REFERENCES: 21 \\
\hline ACQUISITION NO: & HCP/R4024-01 & SOURCE: & \\
\hline
\end{tabular}

KEY WORDS: Energy Options

Fossil. Fuels

Geotherma?

Hydroelectric

Nuclear Power

Solar Power 


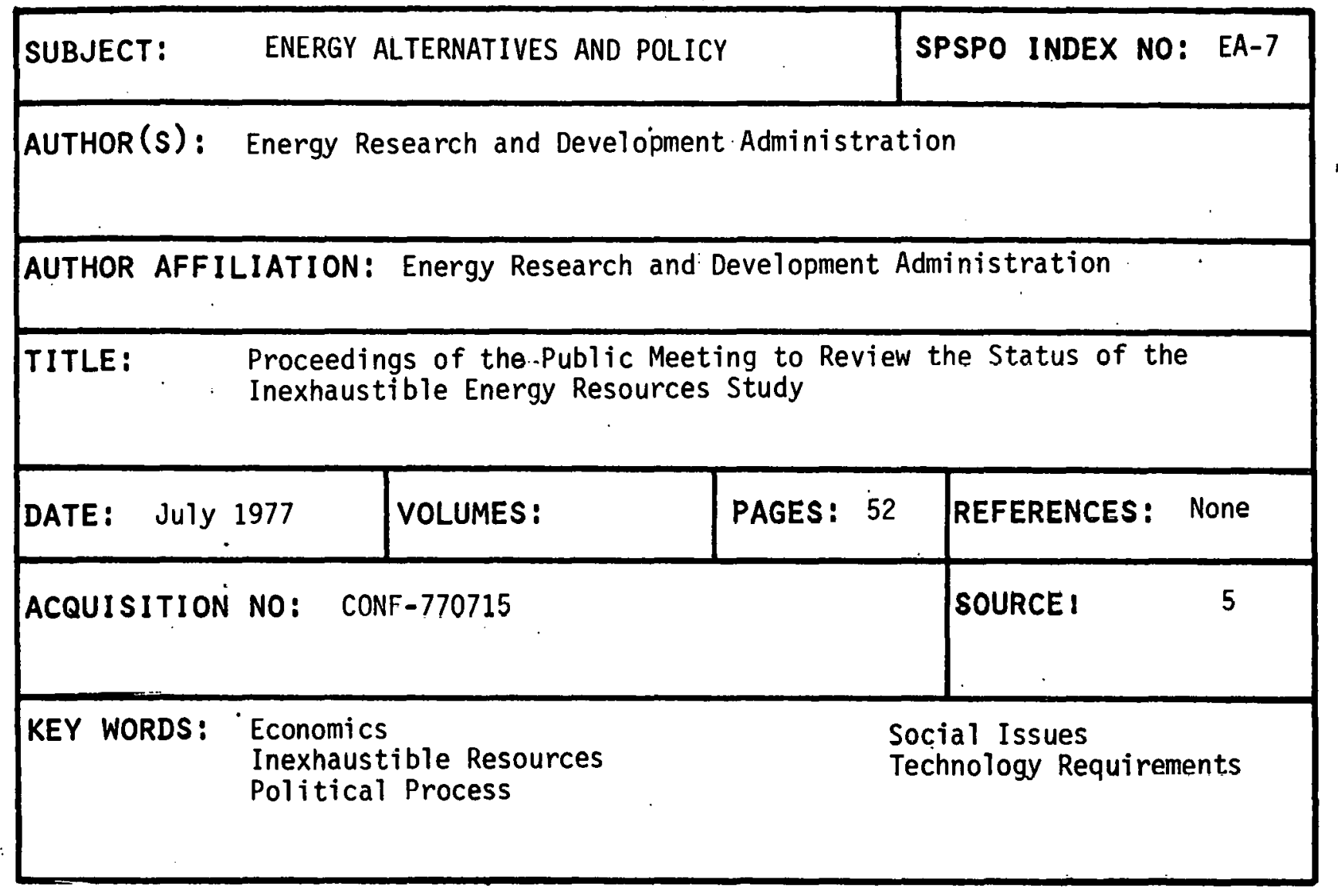

SUBJECT: ENERGY ALTERNATIVES AND POLICY

SPSPO INDEX NO: EA-12

AUTHOR(S): General Accounting Office

AUTHOR APFILIATION: U. S. General Accounting Office

TITLE: Net Energy Analysis: Little Progress and Many Problems

\begin{tabular}{|c|c|c|c|c|}
\hline DATE: August. & 1977 & VOLUMES: & PAGES : 15 & REFERENCES: None \\
\hline \multicolumn{4}{|c|}{ ACQUISITION NO: EMD-77-57 } & SOURCE: 9 \\
\hline KEY WORDS: & \multicolumn{2}{|c|}{$\begin{array}{l}\text { Energy Options } \\
\text { Energy Ratio } \\
\text { Net Energy Analys is }\end{array}$} & \multicolumn{2}{|c|}{ Policy Analysis } \\
\hline
\end{tabular}




\begin{tabular}{|c|c|c|c|c|c|}
\hline \multicolumn{6}{|c|}{ AUTHOR AFFILIATION: Arthur D. Little, Inc. } \\
\hline \multicolumn{6}{|c|}{ Satellite Solar Power--An Option for Power Generation on Earth } \\
\hline \multicolumn{2}{|c|}{ DATE: June 1977} & VOLUMES: & PAGES: 25 & REFERENCES: & $11^{\prime}$ \\
\hline \multicolumn{2}{|c|}{ ACQUISITION NO: } & By Title & $\begin{array}{l}\text { ectrotechical } \\
\text { ss, Moscow) }\end{array}$ & SOURCE: & \\
\hline \multicolumn{3}{|c|}{$\begin{array}{ll}\text { KEY WORDS: } & \text { Concepts } \\
& \text { Economics } \\
& \text { Energy Options }\end{array}$} & \multicolumn{3}{|c|}{$\begin{array}{l}\text { Environmental Effects } \\
\text { Solar Power }\end{array}$} \\
\hline
\end{tabular}

SUBJECT: ENERGY ALTERNATIVES AND POLICY SPSPO INDEX NO: 'EA-13

AUTHOR(s): Grey, J.

AUTHOR AFFILIATION: American Institute of Aeronautics and Astronautics

TITLE: Energy Since the 0i1 War

DATE: November 1975

VOLUMES:

PAGES: 37

REFERENCES: 25

ACQUISITION NO: By Title, Aeronautics and Astronautics

SOURCE: 2

KEY WORDS: Energy Options

Fossit Fuels

Inexhaustible Resources
Nuclear Power

Policy Analysis

Solar Power. 


\begin{tabular}{|l|l|}
\hline SUBJECT: ENERGY ALTERNATIVES AND POLICY & SPSPO INDEX NO: EA-10 \\
\hline AUTHOR(S): Herendeen, R. A. & \\
\hline AUTHOR AFF ILIATION: University of Illinois & \\
\hline TITLE: Two Technologies Near the Net Energy Limit: Gasohol and Solar \\
Satellite Power Station
\end{tabular}

\section{SUBJECT: ENERGY ALTERNATIVES AND POLICY}
$\operatorname{AUTHOR}(s)$ : Herendeen, R. A.
K.ary, T.
Rebitzer, J.

AUTHOR AFFILIATION: University of Illinois

TITLE: Energy Analysis of the Solar Power Satellite

\begin{tabular}{|l|l|l|l|}
\hline DATE: 3 August 1979 & VOLUMES: & PAGES: 4 & REFERENCES: 26 \\
\hline ACQUISITION NO: $\begin{array}{l}0036-8075 / 79 / 0803-0451 \\
\text { Science, Vo1. 205, No. 4405 }\end{array}$ & SOURCE: \\
\hline KEY WORDS: $\begin{array}{l}\text { Energy Options } \\
\text { Energy Ratio } \\
\text { Net Energy Analysis: }\end{array}$ & 7 \\
\hline
\end{tabular}


AUTHOR(S): Kassing, D.

Reinhartz, K. K.

AUTHOR AFFILIATION: European Space Technology and Engineering Center

TITLE: Energy for Europe from Space

\begin{tabular}{|l|l|l|l|}
\hline DATE: 1978 & VOLUMES: & PAGES: 9 & REFERENC̈ES: 20 \\
\hline ACQUISITION NO: By Title, ESA Journal, $\begin{aligned} \text { Vol. } 2, \mathrm{pp} . \\
179-187\end{aligned}$ & SOURCE: \\
\hline KEY WORDS: & $\begin{array}{l}\text { Concepts } \\
\text { EConomics } \\
\text { Energy Options }\end{array}$ & $\begin{array}{l}\text { International Interest } \\
\text { Solar Power }\end{array}$ \\
\hline
\end{tabular}

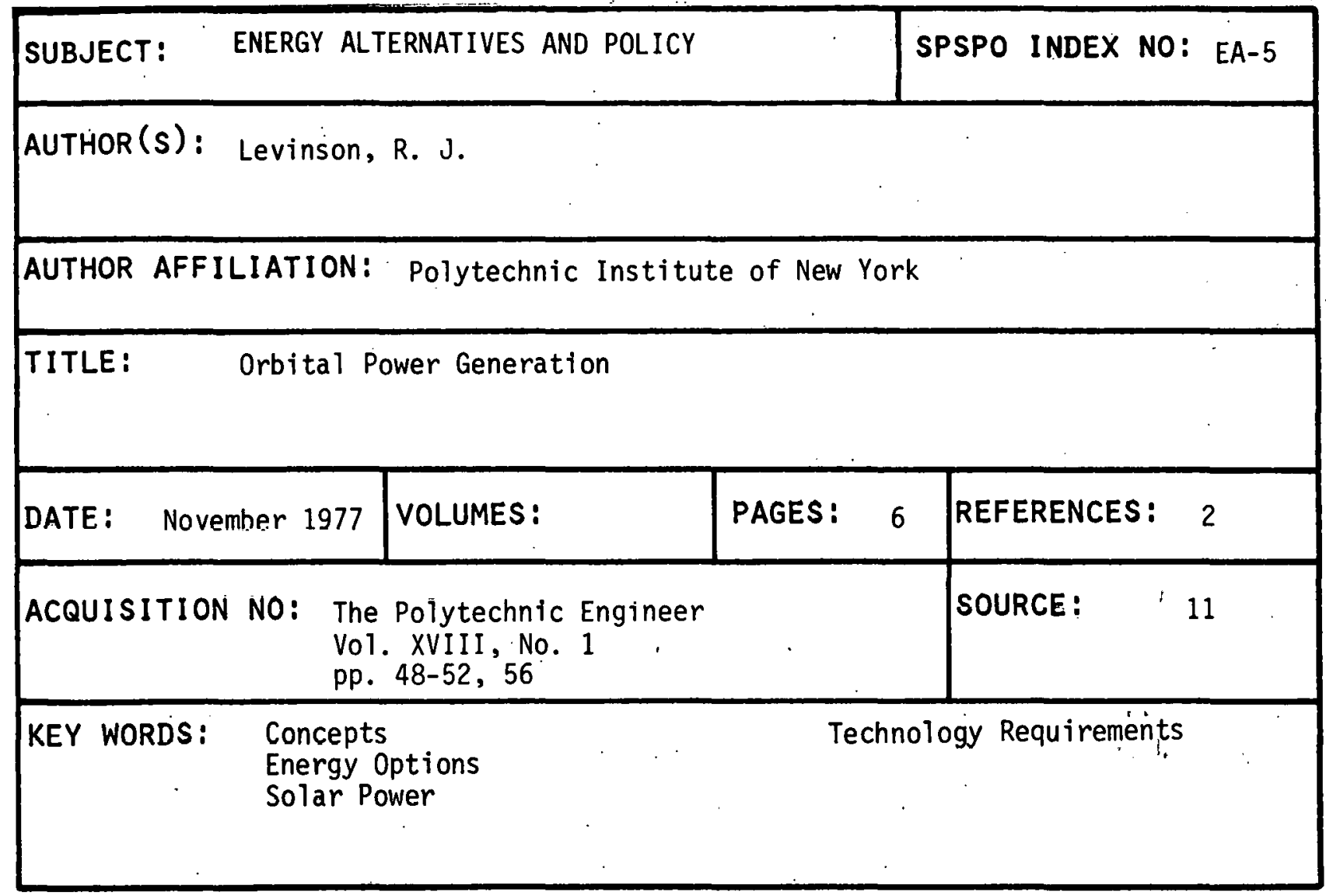




$\begin{array}{ll}\operatorname{AUTHOR}(\mathrm{S}): & \text { Leon, H. I. } \\ & \text { Gorman, R. } \\ & \text { Dawley, R. }\end{array}$

AUTHOR AFFILIATION: TRW Systems Group

TITLE: . Solar Energy Options for Electric Utilities

\begin{tabular}{|ll|}
\hline DATE: Apri1 1976 & VOLUMES: \\
\hline ACQUISITION NO: & PB6-231 \\
& \\
\hline KEY WORDS: & $\begin{array}{l}\text { Comparative Methods } \\
\text { Ocean Conversion } \\
\text { Solar Conversion }\end{array}$
\end{tabular}

\begin{tabular}{ll|l} 
PAGES: & 3 & REFERENCES: 4
\end{tabular}

SOURCE : $\quad 13$

SUBJECT: ENERGY ALTERNATIVES AND POLICY

SPSPO INDEX NO: EA-6

$\operatorname{AUTHOR}(s)$ :

Machol, R. E.

AUTHOR AFFILIATION: Northwestern University

TITLE :

Solar Energy - A. Subjective Assessment for NASA

\begin{tabular}{|c|c|c|c|c|}
\hline DATE: March 1977 & VOLUMES : & PAGES: 23 & REFERENCES : & None \\
\hline ACQUISITION NO: & By Title & & SOURCE: & 4 \\
\hline
\end{tabular}

KEY WORDS: Economics

Solar Power

Technology Requirements 
UTHOR (s): Myers, D. H.

AUTHOR AFFILIATION: U. S. Department of Energy

TITLE: The Energy Research and Development Program of the United States

DATE: December 1978

VOLUMES:

PAGES : 25

REFERENCES: None

ACQUISITION NO: By Title; Anglo-American Workshop on Energy and Aerospace, London

SOURCE : 2

on Energy and Aerospace, London

KEY WORDS: Energy Options

Nuclear Power

Feasibility

Issues

Policy Analysis

Solar Power

SUBJECT: ENERGY ALTERNATIVES AND POLICY

SPSPO INDEX NO: . EA-2

AUTHOR(S): Smith, M.S.

AUTHOR AFFILIATION: Congressional Research Service

Library of Congress

TITLE: Solar Energy from Space: Satellite Power Stations

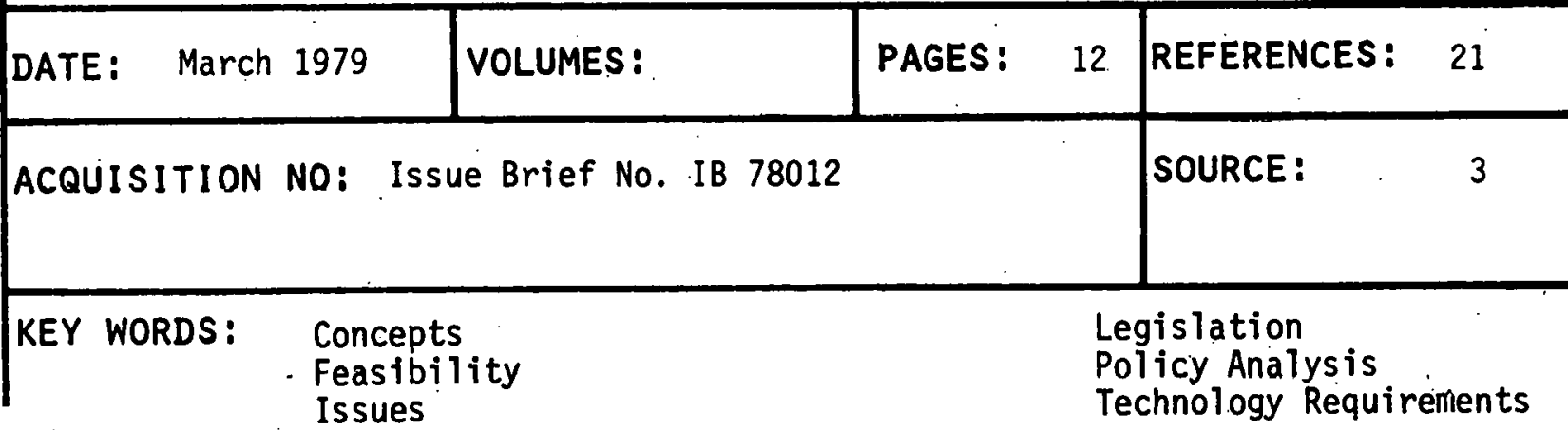


SUBJECT:- ENERGY ALTERNATIVES AND POLICY

AUTHOR(S): Sviedrys, R.

AUTHOR AFFILIATION: Polytechnic Institute of New York

TITLE: $\quad$ The Changing Nature of the Energy Crisis

DATE: November 1977

VOLUMES:

PAGES: 5

REFERENCES : 20

The Polytechnic Engineer

Vol. XVIII, No. 1

pp. $18-21,55$

SOURCE :

11

KEY WORDS: Concepts

Energy Options

Solar Power

Technology Requirements

Fossil Fuels 


\section{ELECTROMAGNETIC COMPATIBILITY \\ (CATEGORY EC)}

This category is concerned with reports and papers which address the possibility that SPS might produce electromagnetic incompatibilities (radio interference) with other electric or electronic systems in any portion of the frequency spectrum, and mitigating practices which might be useful. in the event that interference problems were encountered.

KEY WORDS :

Aeronamy

Antenna Characteristics

Astronomy

Energy Distribution

Equipment Susceptibility

Field Enhancement

Harmonics

Ionosphere Effects

Measurements

Mitigation

Noise Interference

Rules and Regulations

Sidebands

Spurious Emissions

Troposphere Effects
PAGE NUMBER:

81,82

$81,82,83$

82

$82,83,84$

$81,83,85$

82

$81,82,83,85$

84,85

$81,82,83,84$

$81,82,84$

84

$81,82,84$

$83,84,85$

$82,83,84$

85

RELATED CATEGORIES:

Atmospheric Effects (AE)

Environmental Effects (EE)

Power Generation and Transmission (PT)

Systems Engineering (SE) 


\section{THIS PAGE}

\section{WAS INTENTIONALLY LEFT BLANK}




\begin{tabular}{|c|c|c|c|c|}
\hline \multicolumn{5}{|c|}{ AUTHOR AFFILIATION: University of Manchester (England) } \\
\hline \multicolumn{5}{|c|}{$\begin{array}{l}\text { TITLE: Effect of Solar Power Satellite Transmissions on Radio Astronomical } \\
\text { Research }\end{array}$} \\
\hline DATE: Decemt & ber 1978 & VOLUMES: & PAGES: 11 & REFERENCES : 6 \\
\hline \multicolumn{2}{|c|}{ ACQUISITION NO: } & \multicolumn{2}{|c|}{$\begin{array}{l}\text { By Title; Anglo-American Workshop on } \\
\text { Energy and Aerospace, : Royal Aeronautica } \\
\text { Society (London) }\end{array}$} & SOURCE: 2 \\
\hline KEY WORDS : & \multicolumn{4}{|c|}{$\begin{array}{l}\text { Astronomy } \\
\text { Equipment Susceptibility } \\
\text { Rules and Regulations }\end{array}$} \\
\hline
\end{tabular}

TITLE: Environmental Assessment for the Satellite Power System Concept Development and Evaluation Program-Electromagnetic Systems Compatibility.

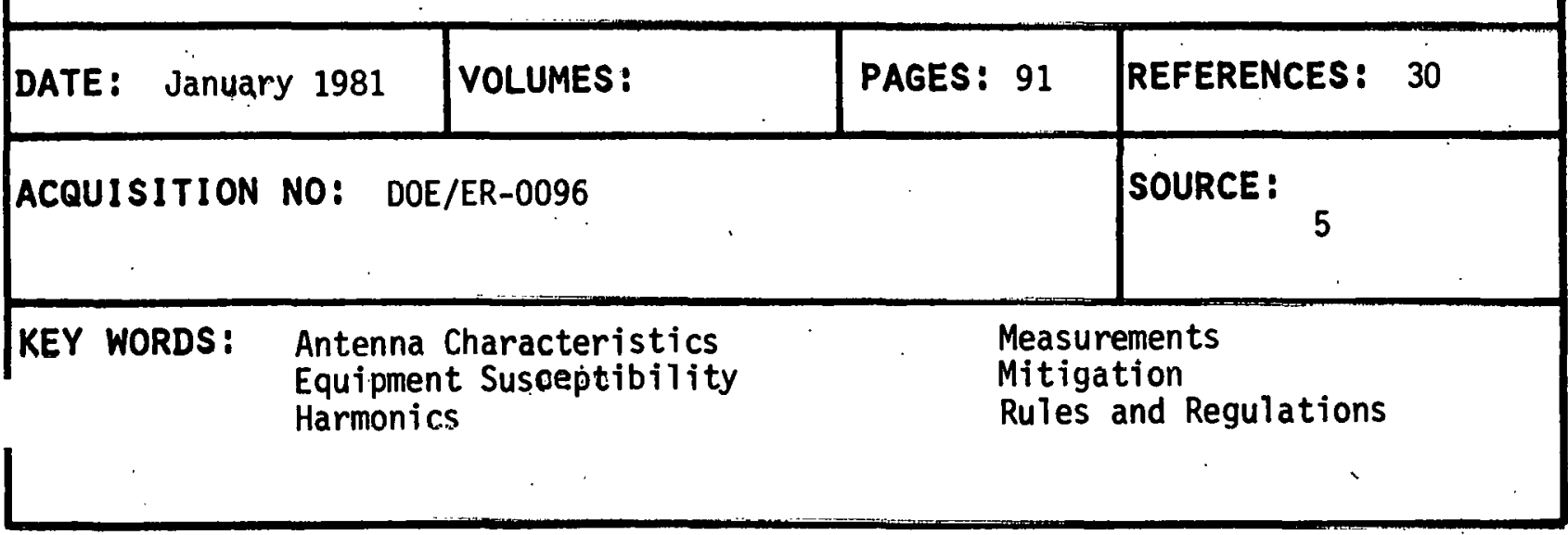


AUTHOR(S): Ekstron, P. A. (Ed.)

Stokes, G. M. (Ed:)

AUTHOR AFFILIATION: Pacific Northwest Laboratory

TITLE: Workshop on Satellite Power Systems (SPS) Effects on Optical and Radio Astronomy

\begin{tabular}{|l|l|l|l|}
\hline DATE: April 1980 & VOLUMES: & PAGES: 265 & REFERENCES: 132 \\
\hline
\end{tabular}

ACQUISITION NO: CONF-79051.43

SOURCE : 5

KEY WORDS: Aeronomy

Antenna Characteristics

As tronomy

Harmonics

Rules and Regulations

Spurious. Emissions

SUBJECT: ELECTROMAGNECTIC COMPATIBILITY

SPSPO INDEX NO: : EC -6

AUTHOR (s): Liebe, H. J.

AUTHOR AFFILIATION: U.S. Department of Commerce

TITLE: Field Maxima Inside Habitable Structures Exposed to $2.45 \mathrm{GHz}$ Plane Wave Radiation

DATE: October 1980

VOLUMES:

PAGES: 45

REFERENCES: 25

ACQUISITION NO: NTIA-80-49

SOURCE： 5

KEY WORDS: Energy Distribution

Mitigation

Field Enhancement

Measurements 
\begin{tabular}{l} 
AUTHOR(S): National Research Council \\
\hline AUTHOR AFFILIATION: National Academy of Sciences
\end{tabular}

TITLE: Radiation Intensity of the PAVE PAWS Radar System

\begin{tabular}{|l|l|l|l|}
\hline DATE: 1979 & VOLUMES: & PAGES: 46 & REFERENCES: 5 \\
\hline ACQUISITION NO: Contract F49620-78-C-0118 & SOURCE : 27 \\
\hline KEY WORDS: Antenna Characteristics & $\begin{array}{l}\text { Measurements } \\
\text { Energy Distribution } \\
\text { Harmoniçs }\end{array}$ & \begin{tabular}{l} 
Spurious Emissions \\
\hline
\end{tabular} \\
\hline
\end{tabular}

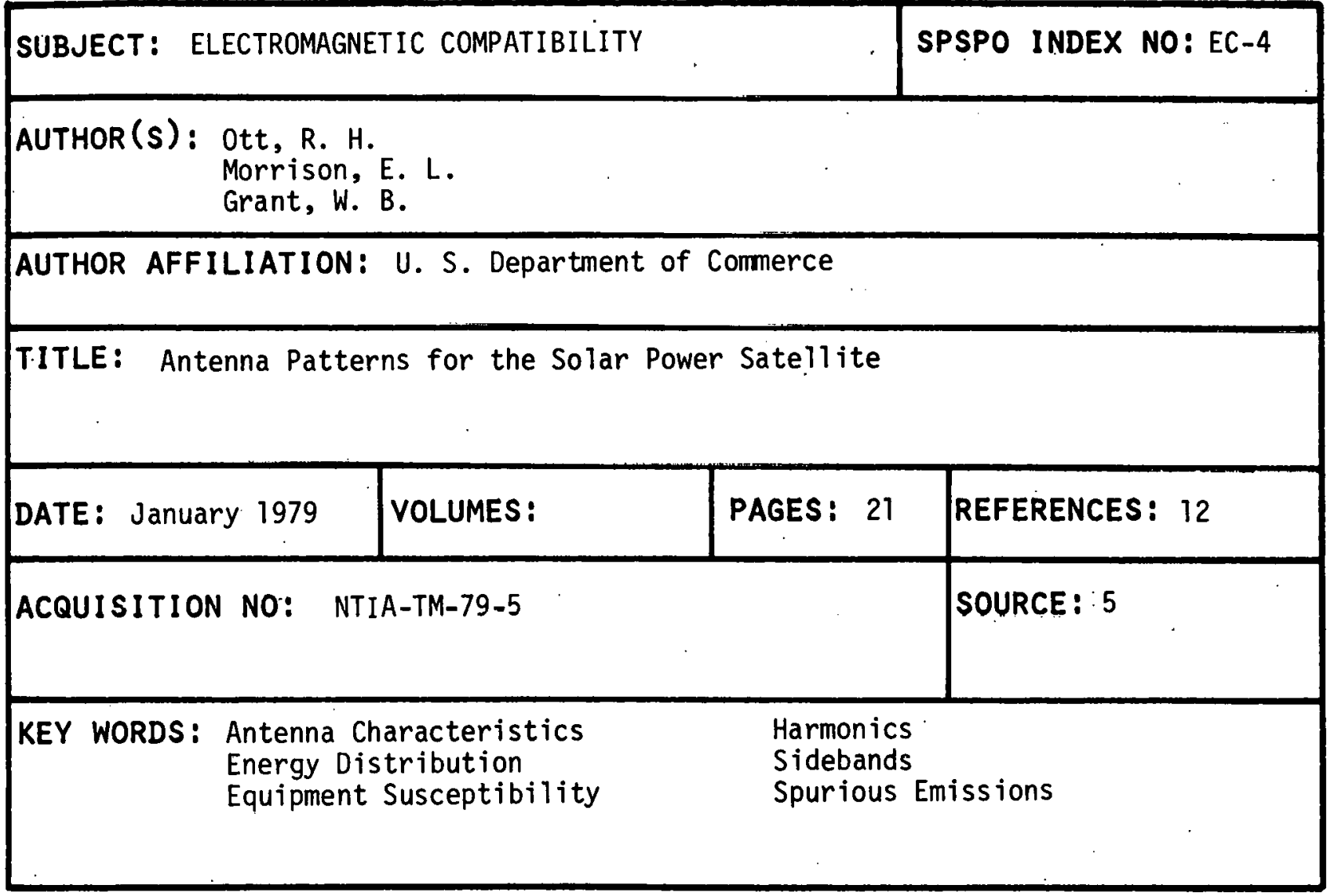


SUBJECT: ELECTROMAGNETIC COMPATIBILITY

SPSPO INDEX NO: EC-1

AUTHOR(s): Pacific Northwest Laboratory

AUTHOR AFFILIATION: Pacific Northwest Laboratory

TITLE: Initial Assessment--Electromagnetic Compatibility Aspects of Proposed SPS Microwave Power Transmission System (MPTS) Operations

\begin{tabular}{|c|c|c|c|c|}
\hline DATE : & February 1978 & VOLUMES: & PAGES: 133 & $\begin{array}{l}\text { REFERENCES: } \\
\text { Bib } 1 \text { lography } \\
\end{array}$ \\
\hline ACQUI & ITION NO: & \multicolumn{2}{|c|}{ PNL-2482 } & SOURCE: 13 \\
\hline & $\begin{array}{l}\text { Energy } \\
\text { Ionosph } \\
\text { Mitigat }\end{array}$ & $\begin{array}{l}\text { Distribution } \\
\text { here Effects } \\
\text { tion }\end{array}$ & & $\begin{array}{l}\text { s and Regulations } \\
\text { bands } \\
\text { ious Emissions }\end{array}$ \\
\hline
\end{tabular}

SUBJECT: ELECTROMAGNETIC COMPATIBILITY

SPSPO INDEX NO: EC-8

$\operatorname{AUTHOR}(s)$ : Spaulding, $\Lambda . D$.

AUTHOR AFFILIATION: U. S. Department of Commerce

TITLE: Man-Made Noise: The Problem and Reconmended Steps Toward Solution

\begin{tabular}{|c|c|c|c|}
\hline DATE: Apr11 1976 & VOLUMES: & PAGES : 185 & REFERENCES : 166 \\
\hline \multicolumn{3}{|c|}{ ACQUISITION NO: OT-76-85 } & SOURCE : 5 \\
\hline \multicolumn{2}{|c|}{$\begin{aligned} \text { KEY WORDS: } & \text { Measurements } \\
& \text { Mitigation } \\
& \text { Noise Interference }\end{aligned}$} & \multicolumn{2}{|c|}{$\begin{array}{l}\text { Rules and Regulations } \\
\text { Spurious Emissions }\end{array}$} \\
\hline
\end{tabular}


$\operatorname{AUTHOR}(S)$ : Telecommunications Sciences, Institute of

AUTHOR AFFILIATION: U. S. Department of Commerce

TITLE: Electromagnetic Compatibility, Tropospheric and Ionospheric Aspects of SPS MPTS Operations

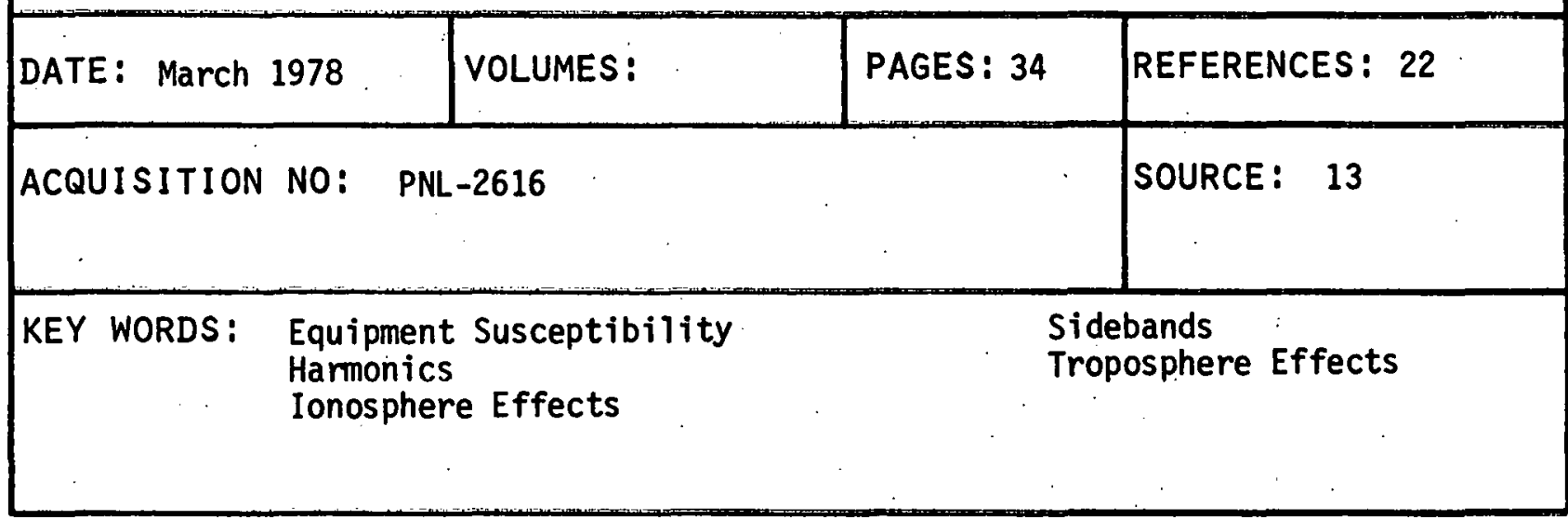




\section{THIS PAGE}

\section{WAS INTENTIONALLY}

LEFT BLANK 


\section{ENVIRONMENTAL EFFECTS \\ (CATEGORY EE)}

Reports, articles and papers in this category cover all environmental concerns about SPS, and generally do not emphasize one particular environmental issue. Multiple issues and inter-relationships between potential effects of SPS on the environment are included in most citations.

KEYY WORDS:

Air Quality

Atmospheric Effects

Atmospheric Heating

Ecosystem Impacts

Electromagnetic Compatibility

Health Maintenance

Hearing Effects

Ionospheric . Effects

Ionospheric Perturbations

Land Use

Laser Effects

Microwave Effects

Noise

Nuclear Effects

Occupational Effects

Operations Effects

ozone

Photovoltaics

Public Welfare

Refiected Light

Safety Issues

Societal Effects

Solid Waste

Space Effects

Space Habitat

Space Health

Terrestrial Effects

Water Resources

Wildlife Effects
PAGE NUMBER:

$92,93,94,95,98,100$

$89,90,91,92,94,96,99$

89

$90,92,93,97,98,100$

$89,91,92,94,97,98,99$

$93,95,97,98,100$

96

$89,90,91,92,94,99$

89

$92,93,94,95,98$

89,90

$89,91,92,94,98,99$

$94,95,96,97,100$

97

$90,91,93,95,96,98,100$

$90,94,95,96,97,98,99$

97

93,98

96,99

90,96

$90,92,93,97,98$

91,$92 ; 98$

$93,95,97$

$89,90,91,92,95,99,100$

95

91,95

$89,91,92,94$

$92,93,94,95$

$89, .94,96$ 
(Environmental Effects

Category EE Continued)

RELATED CATEGORIES:

Atmospheric Effects (AE)

Biologic Effects (BE)

Electromagnetic Compatibility (EC)

Power Generation and Transmission (PT)

Systemis Analyses (SA)

Systems Engineering (SE)

Space Transportation (ST) 
$\operatorname{AUTHOR}(s):$ (1) Abromavage, M. M.
(2) Valentino, A. R.

AUTHOR AFFILIATION: (1) IIT Research Institute

(2) Argonne National Laboratory

TITLE: Environmental Assessment for the Satellite Power System Concept Development and Evaluation Program

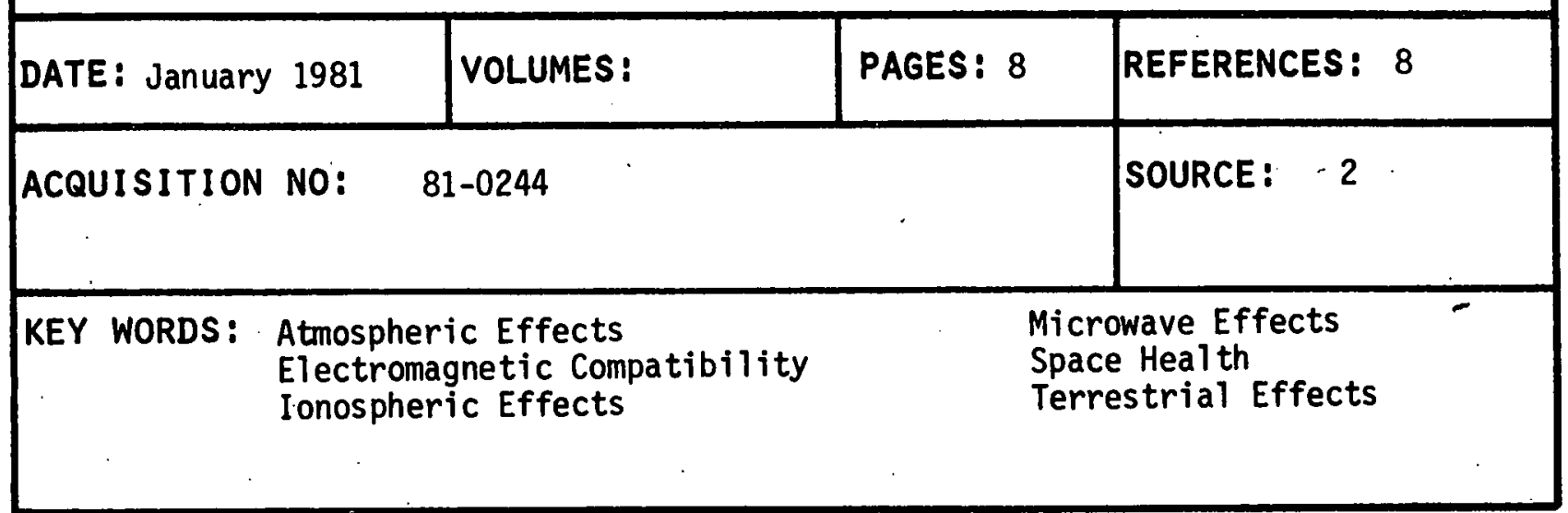

\section{SUBJECT: ENVIRONMENTAL EFFECTS}

SPSPO INDEX NO: EE-3

AUTHOR(S): Beverly; R. E.

AUTHOR AFFILIATION: NONe

TITLE: $\quad$ Satellite Power System (SPS) Laser Environmental Impact Study

\begin{tabular}{|c|c|c|c|c|c|c|}
\hline DATE: Febru & uary 1979 & VOLUMES: & PAGES: & 138 & REFERENCES: & 139 \\
\hline \multicolumn{3}{|c|}{ ACQUISITION NO: NAS8-32475 } & & . & SOURCE: & 4 \\
\hline \multicolumn{3}{|c|}{$\begin{aligned} & \text { KEY WORDS: } \text { Atmospheric Heating } \\
& \text { Ionospheric Perturbations } \\
& \text { Laser Effects }\end{aligned}$} & \multicolumn{4}{|c|}{ Wildlife Effects } \\
\hline
\end{tabular}


SUBJECT: ENVIRONMENTAL EFFECTS

$\operatorname{AUTHOR}(S)$ : Beverly, R. E.

AUTHOR AFFILIATION: Rockwell International

TITLE: Satellite Power Systems (SPS) Laser Studies; Volume I: Laser Environmental Impact Study

\begin{tabular}{|c|c|c|c|}
\hline DATE: November 1980 & VOLUMES: & PAGES : 85 & REFERENCES : 139 \\
\hline ACQUISITION NO: & $\begin{array}{c}\text { A CR } 3346 \\
8-32475\end{array}$ & & SOURCE： 4 \\
\hline $\begin{aligned} & \text { KEYे WORDS: } \text { Atmosphe } \\
& \text { Ecosyst } \\
& \text { Ionosphe }\end{aligned}$ & $\begin{array}{l}\text { c Effects } \\
\text { Impacts } \\
\text { c Effects }\end{array}$ & \multicolumn{2}{|c|}{$\begin{array}{l}\text { Laser Effects } \\
\text { Operations Effects } \\
\text { Safety Issues }\end{array}$} \\
\hline
\end{tabular}

SUBJECT: ENVIRONMENTAL EFFECTS SPSPO INDEX NO: EE-10

AUTHOR(s): Boeing Aerospace Company

AUTHOR AFFILIATION: Boeing Aerospace Company

TITLE: Satellite Power System (SPS) Brightness Due to Reflected Sunlight

DATE: October 1980 VOLUMES: \begin{tabular}{l|l} 
PAGES: 73 & REFERENCES: 8
\end{tabular}

ACQUISITION NO: DOE/ER-0081 SOURCE : 5

KEY WORDS: Operations Effects

Reflected Liaht

Space Effects 
AUTHOR (s): Dickinson, R. M.

AUTHOR AFFILIATION: Jet Propulsion Laboratory

TITLE: $\quad$ Satellite Power System (SPS) Microwave Subsystem Impacts and Benefits

\begin{tabular}{|l|l|l|l|}
\hline DATE: September 1977 & VOLUMES: & PAGES: 104 & REFERENCES: 37 \\
\hline ACQUISITION NO: & $900-800$ & & SOURCE: \\
\hline
\end{tabular}

KEY WORDS: Electromagnetic Compatibility

Ionospheric Effects

Occupational Effects

Microwave Effects

Space Health

Terrestrial Effects

SUBJECT:

ENVIRONMENTAL EFFECTS

SPSPO INDEX NO: EE-6

AUTHOR (S): Energy, U.S. Department of

AUTHOR AFFILIATION: U.S. Department of Energy

TITLE :

Preliminary Environmental Assessment for the Satellite Power System (SPS)

DATE: October 1978 \begin{tabular}{ll|l} 
VOLUMES: & 2 & PAGES: 218.
\end{tabular}

REFERENCES : 122

ACQUISITION NO: DOE/ER-0021/1;.V. 1, Summary (46 pp) DOE/ER-0021/2; V. 2;, Details (172 pp)

SOURCE :

KEY WORDS: 'Atmospheric Effects

Electromagnetic Compatibility

Microwave Effects

Ionospheric Effects

Space Effects

Terrestrial Effects 


\section{SUBJECT: ENVIRONMENTAL EFFECTS}

$\operatorname{AUTHOR}(S)$ : Energy, U.S. Department of

AUTHOR AFFILIATION: U.S. Department of Energy

TITLE: Preliminary Environmental Assessment for the Satellite Power System (SPS), Revision 1

\begin{tabular}{|l|l|l|l|}
\hline DATE: January 1980 & VOLUMES: 2 & PAGES: 252 \\
\hline ACQUISITION NO: & DUE/ER-UU36/1; V. 1, SUmmáry (52 pp) \\
DOE/ER-0036/1; & V. 2, Detai1s (200 pp) \\
\hline
\end{tabular}

KEY WORDS: Atmospheric Effects

Electromagnetic Compatibility

Ionospheric Effects

Microwave Effects

Space Effects

Terrestrial Effects

SUBJECT:

ENVIRONMENTAL EFFECTS

SPSPO INDEX NO: EE-11

$\operatorname{AUTHOR}(s)$ :

Energy Research and Development Administration

AUTHOR AFFILIATION: Energy Research and Development Administration

TITLE: Solar Program Assessment: Environmental Factonrs for Solar Thermal Electric

DATE: March 1977 VOLUMES:

PAGES: 73 REFERENCES:

24

ACQUISITION NO: ERDA 77-47/4 SOURCE : 20

KEY WORDS: Air Quality

Ecosystem Impacts

Lànd Use
Safety: Issues

Societal Effects

Water Resources 
AUTHOR (S): Energy Research and Development Administration

AUTHOR AFFILIATION: Energy Research and Development Administration

TITLE: Solar Program Assessment: Environmental Factors for Photovoltaics

\begin{tabular}{|l|l|l|l|}
\hline DATE: March 1977 & VOLUMES: & PAGES: 62 & REFERENCES: 47 \\
\hline ACQUISITION NO: ERDA $77-47 / 3$ & SOURCE: 20 \\
\hline KEY WORDS: $\begin{array}{l}\text { Air Quality } \\
\text { Health Maintenance } \\
\text { Land Use }\end{array}$ & $\begin{array}{l}\text { Occupational Effects } \\
\text { Photovoltaics } \\
\text { Solid Waste }\end{array}$ \\
\hline
\end{tabular}

SUBJECT: ENVIRONMENTAL EFFECTS

AUTHOR(s): Energy Research and Development Administration

AUTHOR AFFILIATION: Energy Research and Development Administration

TITLE: Solar Program Assessment: Environmental Factors for Solar Total Energy Systems

\begin{tabular}{|c|c|c|c|c|}
\hline DATE: March & 1977 & VOLUMES: & PAGES: 68 & REFERENCES: 17 \\
\hline ACQUISITION & NO: & $D A 77-47 / 5$ & $\leftarrow$ & SOURCE : 20 . \\
\hline KEY WORDS: & \multicolumn{2}{|c|}{$\begin{array}{l}\text { Air Quality } \\
\text { Ecosystem Impacts } \\
\text { Land Use }\end{array}$} & \multicolumn{2}{|c|}{$\begin{array}{l}\text { Safety Issues } \\
\text { Solld Waste } \\
\text { Water Resources }\end{array}$} \\
\hline
\end{tabular}


AUTHOR(s): Environmental Resources Group

AUTHOR AFFILIATION: Environmental Resources Group

TITLE: Prototype Environmental Assessment of the Impacts of Siting and Constructing a Satellite Power System (SPS) Ground Receiving Station (GRS)

\begin{tabular}{|c|c|c|c|c|c|}
\hline \multicolumn{2}{|c|}{ DATE: August' 1980} & \multicolumn{2}{|c|}{ VOLUMES: } & \multirow[t]{2}{*}{ PAGES: 269} & $\begin{array}{l}\text { REFERENCES: } \\
\text { Bibl iography }\end{array}$ \\
\hline \multicolumn{4}{|c|}{ ACQUISITION NO: DOE/ER-0072 } & & SOURCE : 5 \\
\hline KEY WORDS: & $\begin{array}{l}\text { Air Q } \\
\text { Land } \\
\text { Noise }\end{array}$ & & & $\begin{array}{l}\text { Effects } \\
\text { Irces } \\
\text { fects }\end{array}$ & \\
\hline
\end{tabular}

SUBJECT: ENVIRONMENTAL EFFECTS

SPSPO INDEX NO: EE-8

\begin{tabular}{|llll}
\hline AuTHOR (S): & Halverson, S. I.. (1) & Davis, K. & $(3)$ \\
& Rote, D.M. & $(1)$ & White, M. \\
& Rush, C.M. & $(2)$ & Cahili, D. F. (5)
\end{tabular}

AUTHOR AFFILIATION: (1) Argonne National Laboratory; (2) Institute for Telecommunications Sciences; (3) Pacific Northwest Laboratory; (4) Lawrence Berkeley Laboratory: (5) U.S. Environmental Protection Agency

TITLE: Preliminary Assessment of the Environmental Impacts of the Satellite Power System (SPS)

\begin{tabular}{|l|l|l|l|}
\hline DATE: December 1978 & VOLUMES: & PAGES: 17 & REFERENCES: 3 \\
\hline ACQUISITION NO: $\begin{array}{l}\text { By Title (Anglo-American Workshop on } \\
\text { Energy and Aerospace, Royal Aeronaut- } \\
\text { ICal Society, London) }\end{array}$ & SOURCE: \\
\hline KEY WORDS: & $\begin{array}{l}\text { Atmospheric Effects } \\
\text { Electromagnetic Compatibility } \\
\text { Ionospheric Effects }\end{array}$ & $\begin{array}{l}\text { Microwave Effects } \\
\text { Operations Effects } \\
\text { Terrestrial Effects }\end{array}$
\end{tabular}



AUTHOR (S): Kornberg, J.P. Chapman, P. K. Glaser, P. E.

AUTHOR AFFILIATION: A. D. Little, Incorporated

TITLE: Health Maintenance and Health Surveillance Considerations for an SPS Space Construction Community

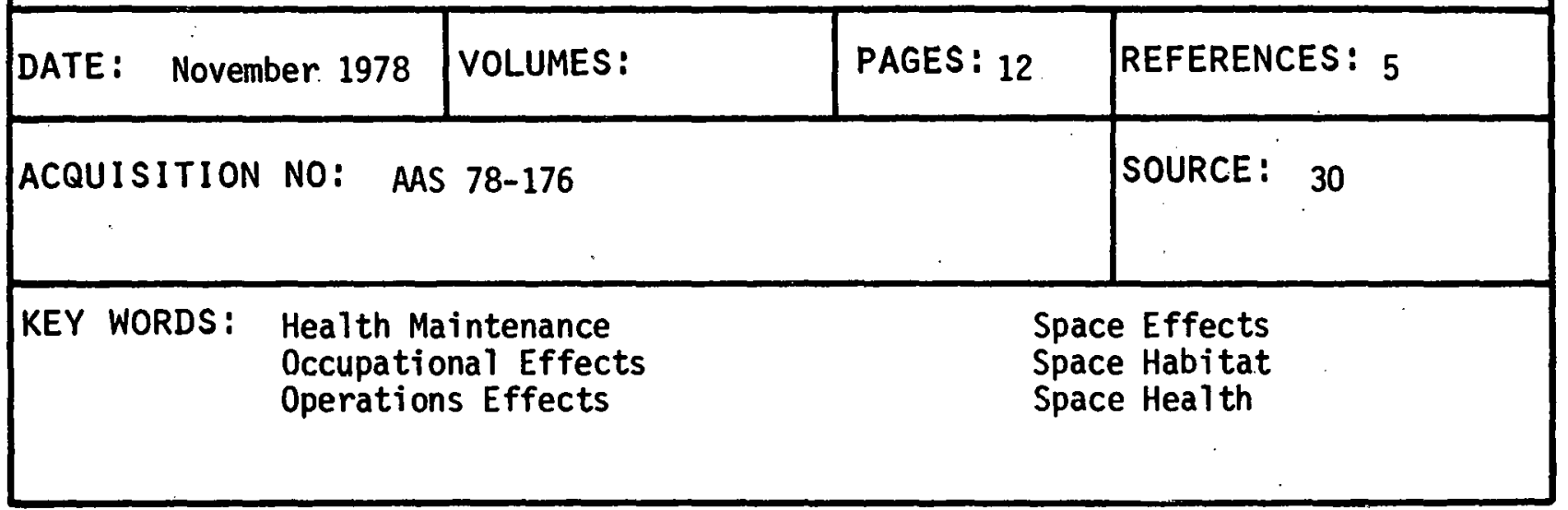

\begin{tabular}{|l|l|}
\hline SUBJECT: $\quad$ ENVIRONMENTAL EFFECTS & SPSPO INDEX NO: EE-4 \\
\hline AUTHOR(S): Livingston; F. R. &
\end{tabular}

\section{AUTHOR AFFILIATION: Jet Propulsion Laboratory} $\begin{array}{ll}\text { TITLE: } & \begin{array}{l}\text { Satellite Power System (SPS) Environmental Impacts Preliminary } \\ \text { Assessment }\end{array}\end{array}$

\begin{tabular}{|l|l|l|l|}
\hline DATE: May 1978 & VOLUMES: & PAGES: 23 & REFERENCES: 10 \\
\hline ACQUISITION NO: JPL-900-822, ReV. A & SOURCE: \\
\hline
\end{tabular}

KEY WORDS: Air Quality Land Use

Solid Waste

Noise

Water Resources 
AUTHOR(S): Livingston, L. E.

AUTHOR AFFILIATION: NASA Johnson Space Center

TITLE: Visibility of Solar Power Satellites from the Earth

\begin{tabular}{|c|c|c|c|c|}
\hline DATE: February 1979 & VOLUMES: & PAGES: $\quad 15$ & REFERENCES: & None \\
\hline ACQUISITION NO: JSC & -14715 & & SOURCE: & 4 \\
\hline
\end{tabular}

KEY WORDS: Operations Effects

Reflected Light

SUBJECT: ENVIRONMENTAL EFFECTS

AUTHOK(s): Pearsons, K. S. White, P. H. Wilby, J. F.

AUTHOR AFFILIATION: Bolt, Berenek and Newman, Inc.

TITLE: An Evaluation of the Noise Impact of Satelite Power System.

DATE: December 1980 VOLUMES:

PAGES: 126 REFERENCES : 35

ACQUISITION NO: LBL-11978 SOURCE： 5

KEY WORDS: Atmospheric Effects Hearing Effects Noise

Occupational Effects

Public Welfare

Wildlife Effects 
AUTHOR (s): Poch, L.A.

AUTHOR AFFILIATION: Argonne National Laboratory

TITLE: A Review of the Nuclear Waste Disposal Problem

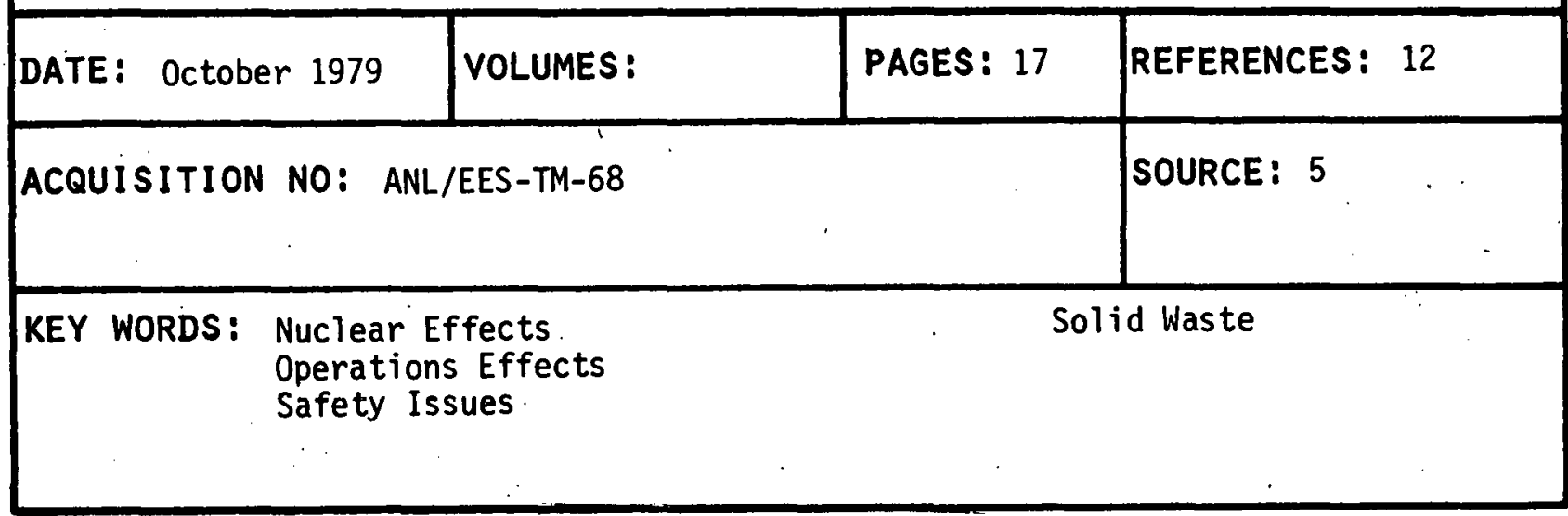

SUBJECT: ENVIRONMENTAL EFFECTS

SPSPO INDEX NO: EE-14

$\begin{array}{llll}\text { AUTHOR }(S): & \begin{array}{l}\text { Scott-Waiton, B. } \\ \text { Clark, K. M. }\end{array} & \text { Jones, D. C. } \\ & \text { Kaplan S. D. } \\ \text { Holt, B. R. } & \text { Krebs, J. S. } & \begin{array}{l}\text { Polson, P. } \\ \text { Shepherd, R. A. }\end{array} \\ & \text { Young, J.R. }\end{array}$

AUTHOR AFFILIATION: SRI International

TITLE: Potential Environmental Effects of 765-kV Transmission Lines: Views Before the New York State Public Service Commission, Cases 26529 and. 26559, 1976-1978

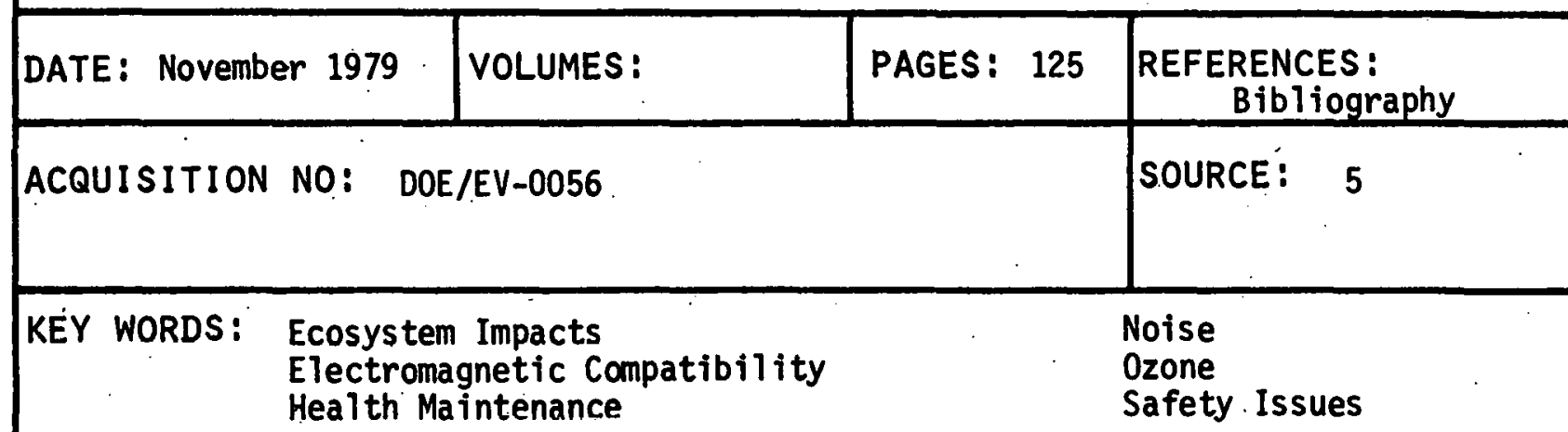




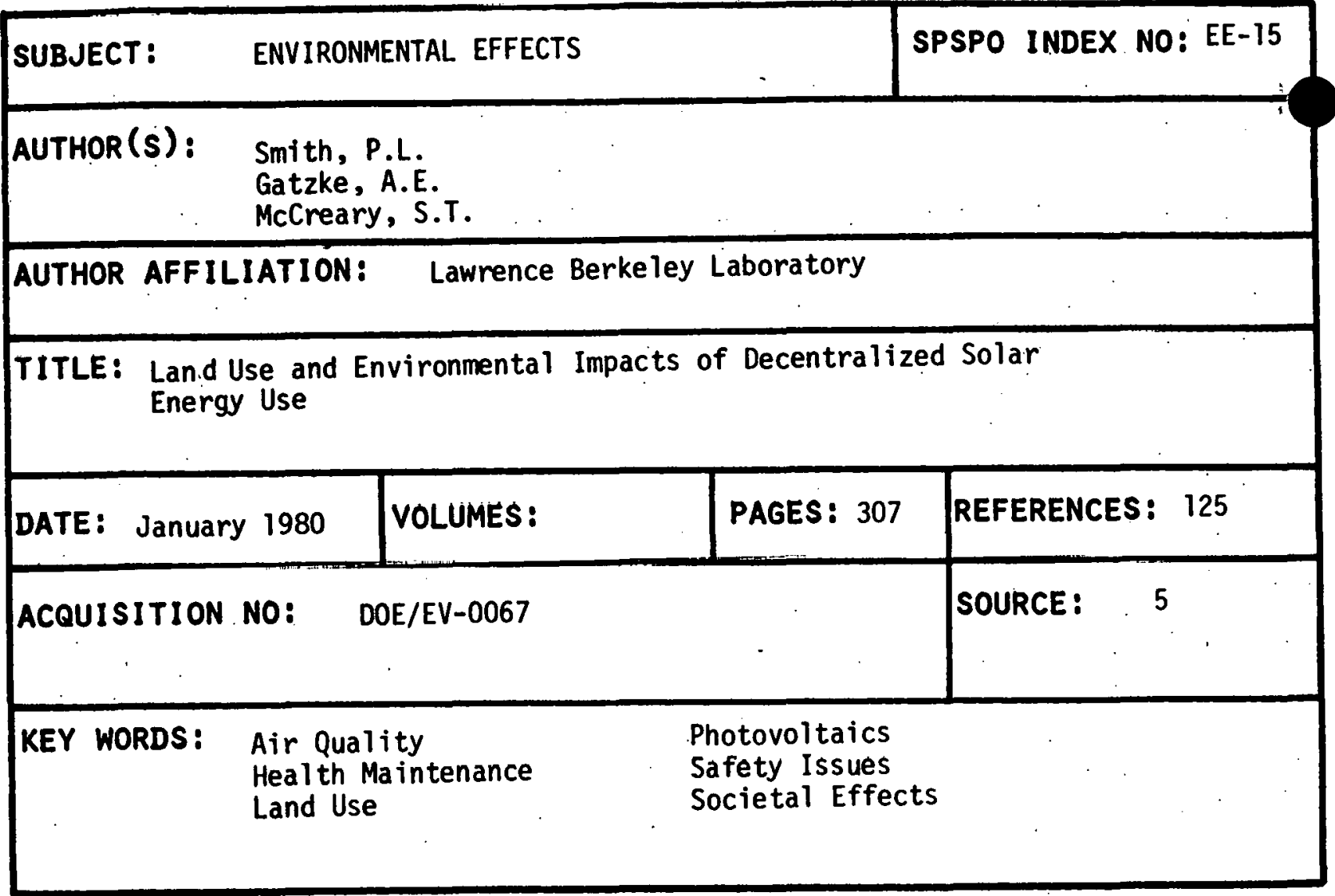

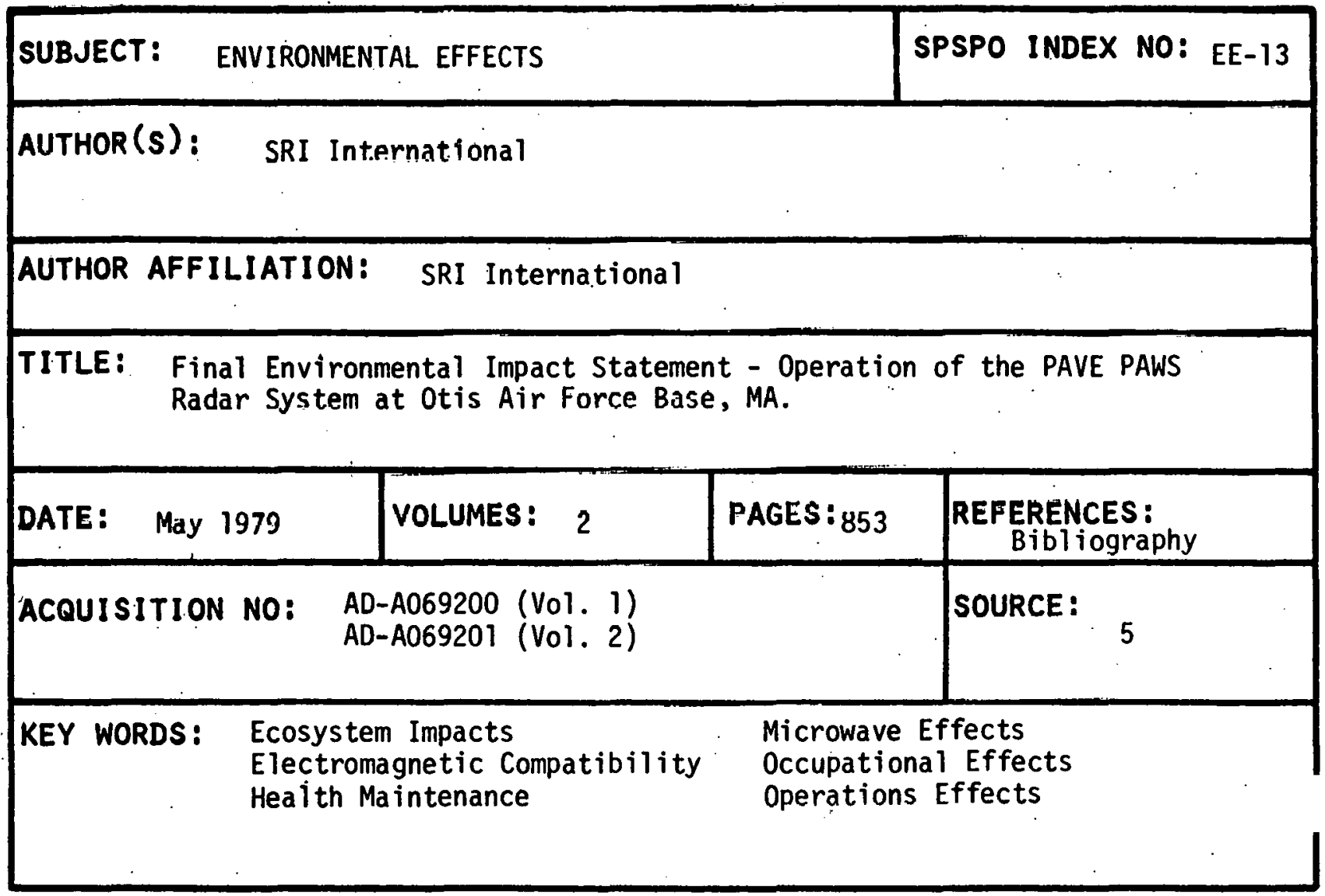


AUTHOR (s):

Valentino, A. R.

\section{AUTHOR AFFILIATION: Argonne National Laboratory}

TITLE: Environmental Assessment for the Satellite Power System (SPS) Concept Development and Evaluation Program (CDEP)

\begin{tabular}{|l|l|l|l|}
\hline DATE: August 1980 & VOLUMES: & PAGES: 127 & REFERENCES: 81 \\
\hline ACQUISITION NO: $\quad$ DOE/ER-0069 & SOURCE: 5 \\
\hline KEY WORDS: $\begin{array}{l}\text { Atmospheric Effects } \\
\text { Electromagnetic Compatibility } \\
\text { Ionospheric Effects }\end{array}$ & $\begin{array}{l}\text { Microwave Effects } \\
\text { Operations Effects } \\
\text { Space Health }\end{array}$ \\
\hline
\end{tabular}

SUBJECT: ENVIRONMENTAL EFFECTS

SPSPO INDEX NO: EE-21

$\operatorname{AUTHOR}(s):$ (1) Valentino, A. R.

(2) Abromavage, M. M.

AUTHOR AFFILIATION: (1) Argonne National Laboratory

(2) IIT Research Institute

TITLE: Environmental Assessment of the Satellite Power System Microwave Power Transmission System

\begin{tabular}{|l|l|l|l|}
\hline DATE: December 1980 & VOLUMES: & PAGES: 4 & REFERENCES: 4 \\
\hline ACQUISITION NO: $\begin{array}{l}\text { By Title; National Telecommunications } \\
\text { Conference (Houston) }\end{array}$ & SOURCE: 6 \\
\hline KEY WORDS: & $\begin{array}{l}\text { Atmospheric Heating } \\
\text { Electromagnetic Compatibility } \\
\text { Innospheric Effects }\end{array}$ & $\begin{array}{l}\text { Microwave Effects } \\
\text { Occupational Effects } \\
\text { Public Welfare }\end{array}$ \\
\hline
\end{tabular}


$\operatorname{AUTHOR}(s)$ : White, M. R.

AUTHOR AFFILIATION: Lawrence Berkeley Laboratory

TITLE: Environmental Assessment for the Satellite Power System Concept Development and Evaluation Program - Nonmicrowave Heal th and Ecological Effects

\begin{tabular}{|l|l|l|l|}
\hline DATE: November 1980 & VOLUMES: & PAGES: 86 & REFERENCES: 51 \\
\hline
\end{tabular}

ACQUISITION NO: DOE/ER-0089

SOURCE : 5

KEY WORDS: Air Quality

Ecosystem Impacts

Heath Maintenance

Noise

Occupational Effects

Space Effects 


\section{ECONOMICS AND FINANCES \\ (CATEGORY EF)}

This category of documents includes reports and papers which deal with the costs and economics involved in the development of SPS, and possible ways in which the development program might be financed in the future. Market potential, production costs and economic risks are included.

KEY WORDS:

Consumption Patterns

Cost Model

Cost Uncertainty

Demand Rates

Disposal costs

Emerging Technologies

Energy Costs

Energy Policy

Financial Requirements

Maintenance Cost

Market Potential

Operations Costs

Production Cost

Returns

Risks

Rewards

Satell1te Salvaye

Uncertainties
PAGE NUMBER:

103

104,105

$104,105,106,107,108$

103,105

103

$103,105,106$

$105,106,108$

103

$106,107,108$

$104,105,108$

103

$104,105,106,107$

$104,105,106,107,108$

106,107

$104,105,106,107$

106

103

105,108

\section{RELATED CATEGORIES:}

Comparative Analyses (CA)

Energy Alternatives and Policy (EA)

Resources and Materials (RM)

Systems Analyses (SA)

Social. Issues (SI) 
THIS PAGE

WAS INTENTIONALLY

LEFT BLANK 
SUBJECT: ECONOMICS AND FINANCES

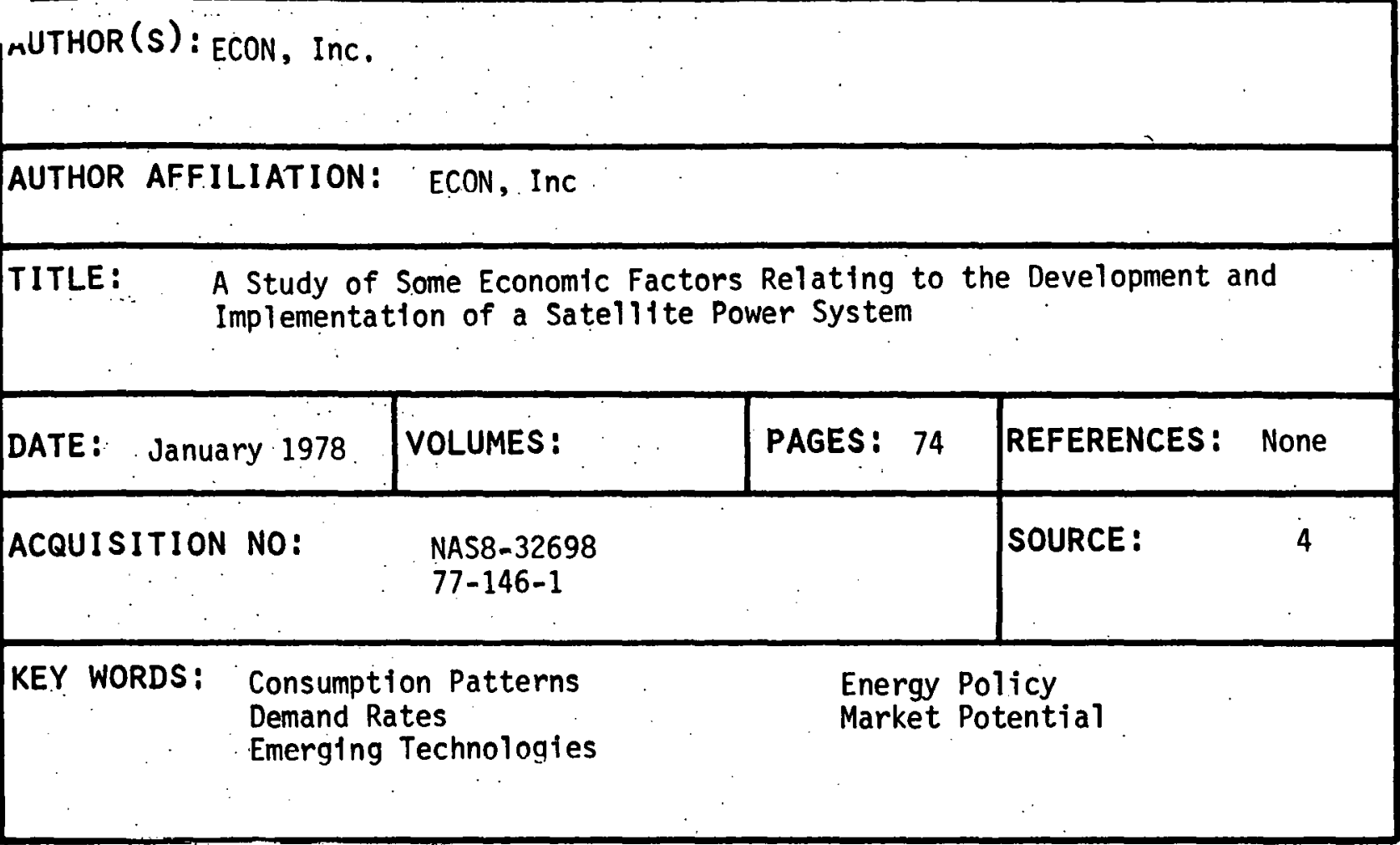

\section{SUBJECT: ECONOMICS AND FINANCES}

AUTHOR (S): ECON, InC.

AUTHOR AFFILIATION: ECON, InC.

TITLE: Satellite Power System Salvage and Disposal Alternatives

\begin{tabular}{|c|c|c|c|}
\hline DATE: November 198? & VOLUMES: & PAGES: 90 & REFERENCES: 8 \\
\hline ACQUISITION NO: N & $\begin{array}{l}\text { CR } 3349 \\
8-.33783\end{array}$ & & SOURCE: 4 \\
\hline
\end{tabular}

KEY WORDS: Disposal Costs

Satellite Salvage 
AUTHOR(S): Hazelrigg, G. A.

AUTHOR AFFILIATION: ECON, Incorporated

TITLE:

Space-Based Solar Power Conversion and Delivery Systems Study

\begin{tabular}{|l|l|}
\hline DATE: June 1976 & VOLUMES: 2 \\
\hline
\end{tabular}

ACQUISIIIUN NO:

$$
\begin{aligned}
& \text { NNS8 } 31308 \\
& 76-145-2
\end{aligned}
$$$$
\text { Vol. } 1 \text { - Summary (32 pp.) }
$$$$
\text { Vol. } 2 \text { - Economic Analysis (128 pp.) }
$$

PAGES: 160

\begin{tabular}{|l} 
SOURCE: \\
REFEREN \\
\hline ions Cost \\
tion Cost
\end{tabular}

Operations Cost

Production Cost
KEY WORDS: Cost Mode1

Cost Uncertainty

Maintenance Cost

SUBJECT: ECONOMICS AND FINANCES

AUTHOR(s): Hazelrigg, G. A.

AUTHOR AFFILIATION: ECON, Incorporated

TITLE: The Economic Viability of Pursuing a Space Power System Concept

DATE: March-Apri1 1977 VOLUMES:

\begin{tabular}{l|l} 
PAGES: 9 & REFERENCES: 3
\end{tabular}

ACQUISITION NO: By Title, AIAA Journal of Energy

SOURCE : 2

KEY WORDS: Cost Model

Cost Uncertainty

Maintenance Costs
Operations Costs

Production Cost

Risks 


\begin{tabular}{|l|l|l|}
\hline AUTHOR (S): Hazelrigg, G. A. \\
\hline AUTHOR AFFILIATION: ECON, Inc. \\
\hline TITLE: Costing the Satellite Power System & PAGES: 21 & REFERENCES: 6 \\
\hline DATE: November 1978 & VOLUMES: & SOURCE: 30 \\
\hline ACQUISITION NO: AAS 78-166 & & . \\
\hline \multicolumn{3}{|l|}{$\begin{array}{l}\text { Production Cost } \\
\text { Risks } \\
\text { Uncertainties }\end{array}$} \\
\hline KEY WORDS: Cost Uncertainty \\
Emerging Technologies
\end{tabular}

SUBJECT: ECONOMICS AND FINANCES

\begin{tabular}{|c|c|c|c|}
\hline $\begin{array}{rr}\operatorname{AUTHOR}(\mathrm{S}): & (1) \\
& (1) \\
(2)\end{array}$ & $\begin{array}{l}\text { Hub, K. } \\
\text { Conley, L. } \\
\text { Buehring, W. }\end{array}$ & $\begin{array}{l}(2) \\
(1)\end{array}$ & $\begin{array}{l}\text { Rowland, B. } \\
\text { Stephenson, M. }\end{array}$ \\
\hline
\end{tabular}

AUTHOR AFFILIATION: (1) Argonne National Laboratory

(2) University of Wiscons in

TITLE: Social Cost Studies Program - Electrical Utility Generating System Reliability Analys is Code, SYSREL

\begin{tabular}{|c|c|c|c|c|}
\hline DATE: Septemt & ber 1975 & VOLUMES: & PAGES: 151 & REFERENCES : 5 \\
\hline \multicolumn{4}{|c|}{ ACQUISITION NO: ANL/AA-4 } & SOURCE: 5 \\
\hline \multicolumn{3}{|c|}{$\begin{aligned} \text { KEY WORDS: } & \text { Cost Mode1 } \\
& \text { Cost Uncertainty } \\
& \text { Demand Rates }\end{aligned}$} & & $\begin{array}{l}\text { Energy Costs } \\
\text { Maintenance Cost } \\
\text { Operations Cost }\end{array}$ \\
\hline
\end{tabular}




\section{SUBJECT: : ECONOMICS AND FINANCES}

$\operatorname{AUTHOR}(S)$ : Kierolff, H. E.

AUTHOR AFFILIATION: PRC Energy Analys is Company

TITLE: "Satellite Power System (SPS) Financial/Management Scenarios

\begin{tabular}{|l|l|l|l|}
\hline DATE: October 1978 & VOLUMES: & PAGES: 62 & REFERENCFS: 77 \\
\hline ACQUISITION NO: & HCP/R-4024-13 & & SOURCE : \\
\hline
\end{tabular}

KEY WORDS: Risks Uncertainties

Returns

Rewards

SUBJECT: ECONOMICS AND FINANCES

SPSPO INDEX NO: EF-9

$\operatorname{AUTHOR}(S)$; Nansẹn, R. H.

Johnson, 0 . E.

AUTHOR AFFILIATION: Boeing Aerospace Corporation

TITLE: Economic Aspects of Energy From Space

DATE: October 1979

VOLUMES:

PAGES: 26

REFERENCES: None

ACQUISITION NO: $79-236$

SOURCE : 2

KEY WORDS: Cost Uncertainty

Emerging Technologies

Energy Costs
Financial Requirements

Operations Cost

Production Cost 
SUBJECT: ECONOMICS AND FINANCES

SPSPO INDEX NO: $\dot{E} F-6$

AUTHOR(S): Rossin, A. D.

Rieck, T. A.

AUTHOR AFFILIATION: Commonwealth Edison Company

TITLE: Economics of Nuclear Power

\begin{tabular}{l|l} 
DATE: August $1978 \quad$ VOLUMES:
\end{tabular}

\begin{tabular}{l|l} 
PAGES: 8 & REFERENCES: 24
\end{tabular}

ACQUISITION NO: $0036-8075 / 78 / 0818-0582501$

SOURCE : : 7

KEY WORDS: Cost Uncertainty

Financial Requirements

Operations Costs

Production Cost

Returns

Risks

SUBJECT: ECONOMICS AND FINANCES

SPSPO INDEX NO: EF-4

AUTHOR(S): Vajk, J.P.

AUTHOR AFFILIATION: Science Applications, Inc.

TITLE: $\quad$ Satellite Power System (SPS) Financial/Management Scenarios

\begin{tabular}{|l|l|l|lc|}
\hline DATE: OCtober 1978 & VOLUMES: & PAGES: i3 & REFERENCES: & 10 \\
\hline ACQUISITION NO: & HCP/R-4024-03 & & SOURCE: & 5 \\
\hline
\end{tabular}

KEY WORDS: Financial Requirements 
$\operatorname{AUTHOR}(S)$ : Woodcock, G. R.

AUTHOR AFFILIATION: Boeing Aerospace Company

TITLE: SPS Cost Considerations

\begin{tabular}{|l|l|l|l|}
\hline DATE: JUTy-August 1978 & VOLUMES: . & PAGES: 7 & REFERENCES: None \\
\hline ACQUISITION NO: By Title; Journal of Energy, V01: 2, & SOURCE: \\
\begin{tabular}{l} 
No. 4, pp. 196-202 \\
\hline
\end{tabular}
\end{tabular}

KEY WORDS: Cost Uncertainty Energy Costs

Financial Requirements

Maintenance cost

Production cost

Uncertainties 


\section{PROGRAM PLANS \\ (CATEGORY PP)}

Program plans developed for the overall SPS Concept Development and Evaluation Program and its various elements are included in this category. Not all plans were implemented, and some future planning is included. Plans for some other energy technologies are listed, as they are pertinent to assessing the SPS energy concept.

KEY WORDS:

Assessment Concept

Atmospheric Effects

Biomass

Comparative Assessment

Concept Definition

Concept Selection

Cost Analys is

Decision Process

Ecology

Environmental Assessment

Environmental Integration

Environmental Objectives

Health Effects

Key Issues

Lessons Learned

Microwave Effects

Ocean Therma?

Photovoltaics

Program 0bjectives

Reference System

Resources

Safety Effects

Societal Assessment

Socio-Economics

Solar Thermal

Space Technology.

Space Transportation
PAGE NUMBER:

111

114,116

113

$112,113,114,115$

112

111

111

115

116

$112,114,115,116$

111

$111,112,113,117$

116,117

$111,112,113$

115

116

113

112,113

115

111,114

117

$112,113,117$

114,116

$112,115,117$

112,113

115

111,115 
(Program Plans

Category PP continued)

. KEY WORDS:

System Operations

Systems Definition

Technology Requirements

Wind Energy
PAGE NUMBER:

116,117

$112,114,115$

116

113

RELATED CATEGORIES:

Atmospheric Effects (AE)

Biologic Effects (BE)

Comparative Analyses (CA)

Electromagnetic Compatibility (EC)

Environmental Effects (EE)

Systems Analyses (SA)

Systems Engineering (SE)

Social Issues (SI)

Space Transportation (ST) 
AUTHOR(s): Aerospace Corporation

AUTHOR AFFILIATION:

Aerospace Corporation TITLE: $\begin{aligned} & \text { Systems Definition Plan for the ERDA Satellite Power Station } \\ & \text { Program }\end{aligned}$

\begin{tabular}{|l|l|l|lc|}
\hline DATE: February 1977 & VOLUMES: & PAGES: 50 & REFERENCES: 8 \\
\hline ACQUISITION NO: & SOURCE: & 13 \\
\hline KEY WORDS: & $\begin{array}{l}\text { Concept Selection } \\
\text { Cost Analysis } \\
\text { Reference System }\end{array}$ & & \\
\hline
\end{tabular}

\section{SUBJECT: PROGRAM PLANS}

$\begin{aligned} & \operatorname{AUTHOR}(s): \text { Bloomquist, C. E. } \\ & \text { Philipson, L. L. }\end{aligned}$

AUTHOR AFFILIATION: PRC Systems Sciences Company

TITLE:

SPS Environmental Assessment Plan (SEAP)

\begin{tabular}{|l|l|l|l|}
\hline DATE: November 1976 & VOLUMES: & PAGES: 53 & REFERENCES: 2 \\
\hline ACQUISITION NO: $\quad \begin{array}{l}\text { ERDA-EG-77-X-10-0794 } \\
\text { PRC R-1847 }\end{array}$ & SOURCE: \\
& & & 13 \\
\hline
\end{tabular}

KEY WORDS: Assessment Concept Cost Analysis

Environmental Integration

Environmental objectives

Key Issues 
SUBJECT: $\quad$ PROGRAM PLANS

SPSPO INDEX NO: PP-5

AUTHOR (s): Energy, U.S. Department of

National Aeronautics and Space Administration

AUTHOR AFFILIATION: U.S. Department of Energy

National Aeronautics and Space Administration

TITLE: $\quad$ Satellite Power System (SPS) Concept Development and Evaluation Program Plan (July 1977 - August 1980)

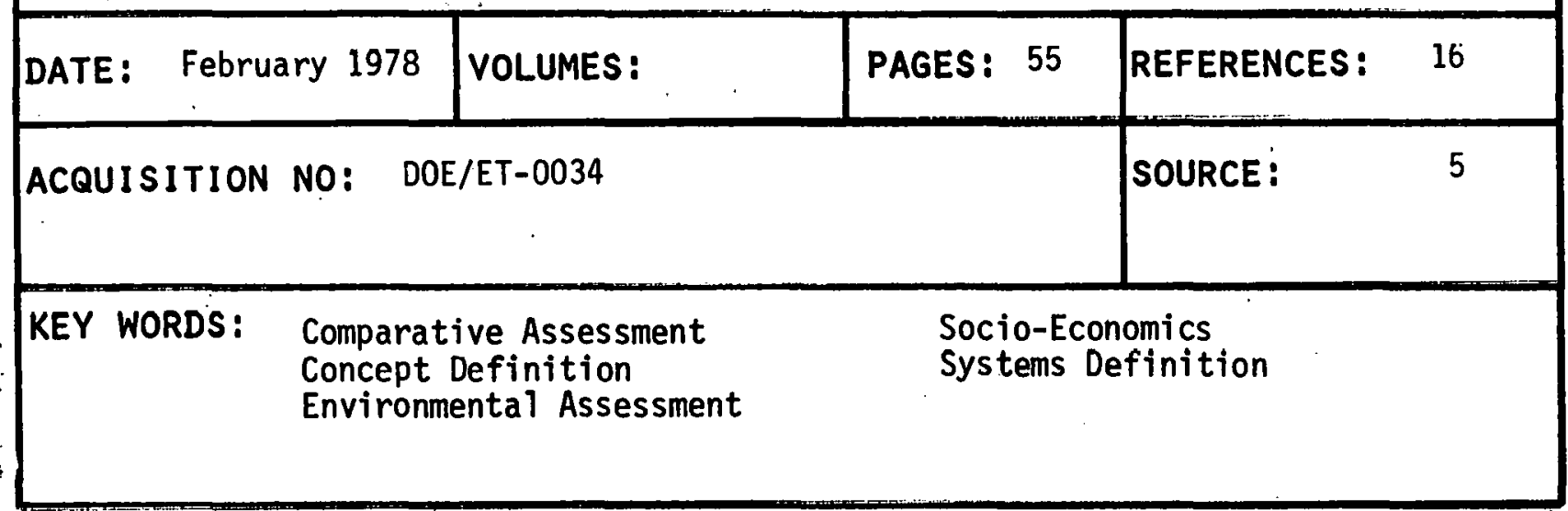

SUBJECT: : PROGRAM PLANS

SPSPO INDEX NO: PP-13

AUTHOR (s): Energy, U.S. Department of Energy

AUTHOR AFFILIATION: U.S. Department of Energy

TITLE: Environmental Development Plan (EDP) for Solar Thermal Power Systems

\begin{tabular}{|c|c|c|c|c|c|}
\hline DATE: March & 1978 & VOLUMES: & PAGES: 60 & \multicolumn{2}{|c|}{$\begin{array}{c}\text { REFERENCES : } \\
\text { Biblioqraphy }\end{array}$} \\
\hline ACQUISITION & NO: & \multicolumn{2}{|l|}{ DOE/EDP-0004 } & SOURCE: & 5 \\
\hline KEY WORDS: & $\begin{array}{l}\text { Envi } \\
\text { Envi } \\
\text { Key }\end{array}$ & $\begin{array}{l}\text { ental Assessment } \\
\text { ental Objectives } \\
\text { es }\end{array}$ & \multicolumn{3}{|c|}{$\begin{array}{l}\text { Photovoltaics } \\
\text { Safety Effects } \\
\text { Solar Thermal }\end{array}$} \\
\hline
\end{tabular}


AUTHOR (s): Energy, U.S. Department of

AUTHOR AFFILIATION: U.S. Department of Energy

TITLE: Environmental Development Plan (EDP) for Photovoltaics

\begin{tabular}{|c|c|c|c|}
\hline DATE: March 1978 & VOLUMES: & PAGES: 51 & $\begin{array}{l}\text { REFERENCES: } \\
\text { Bibliography }\end{array}$ \\
\hline ACQUISITION NO: & DOE/EDP-0003 & & SOURCE: 5 \\
\hline
\end{tabular}

KEY WORDS: Environmental Assessment Environmental Objectives Key Issues
Photovoltaics

Safety Effects

Solar Thermal

$\begin{array}{ll}\text { SUBJECT: } & \text { PROGRAM PLANS } \\ \text { AUTHOR }(S): & \text { Energy, U.S. Department of }\end{array}$

AUTHOR AFFILIATION: U.S. Department of Energy

TITLE: Guide to Solar Energy Programs

\begin{tabular}{|l|l|l|l|}
\hline DATE: March 1978 & VOLUMES: & PAGES: 66 & REFERENCES: None \\
\hline ACQUISITION NO: & DOE/ET-0036 & SOURCE: \\
\hline KEY WORDS: $\begin{array}{l}\text { Blomass } \\
\text { Ocean Thermal } \\
\text { Photovoltatcs }\end{array}$ & $\begin{array}{l}\text { Solar Thermal } \\
\text { Wind Energy }\end{array}$ \\
\hline
\end{tabular}




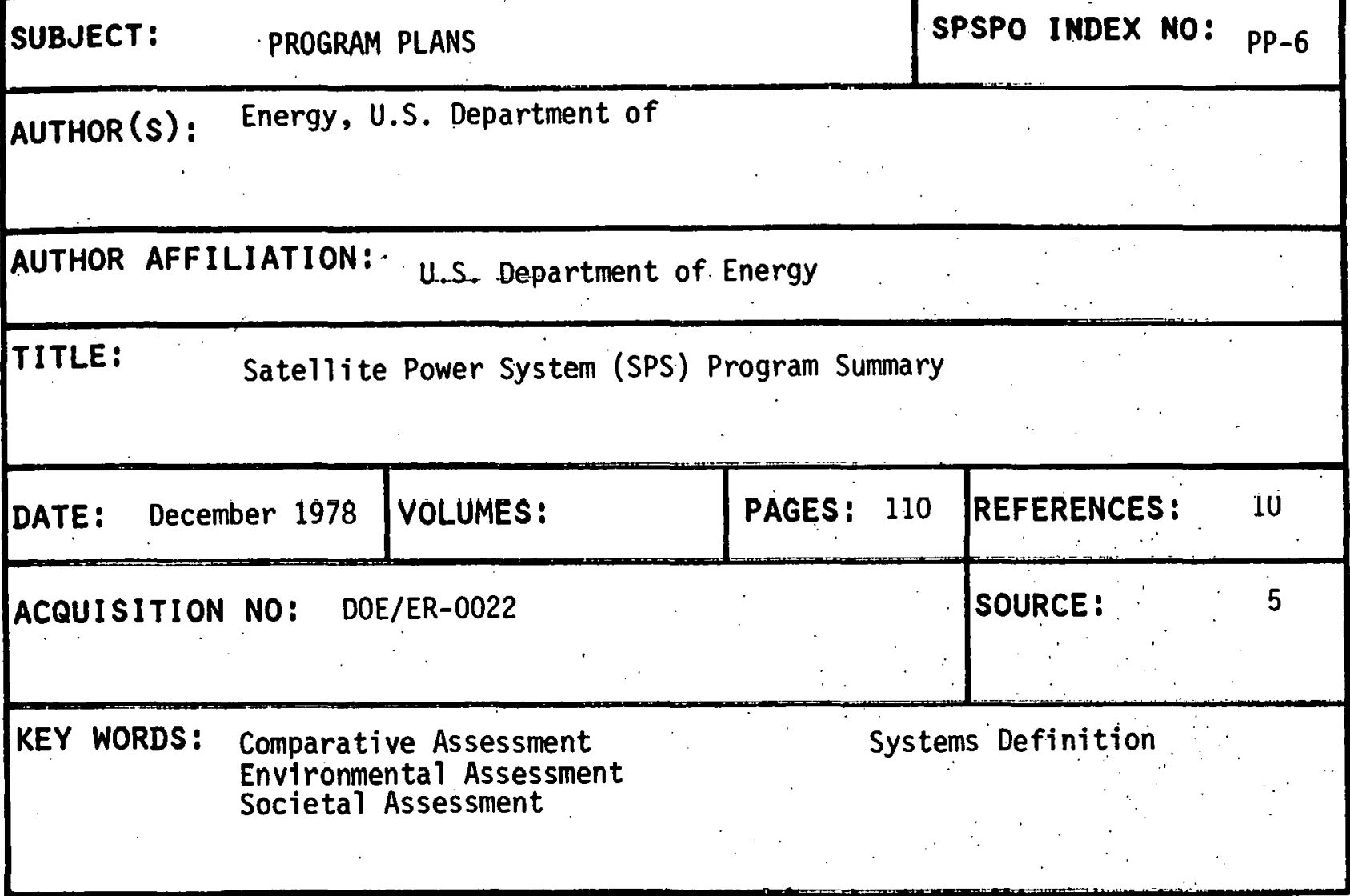

\begin{tabular}{|c|c|c|c|c|}
\hline SUBJECT: & \multicolumn{3}{|c|}{ PROGRAM PLANS } & SPSPO INDEX NO: PP-9. \\
\hline \multicolumn{5}{|c|}{ AUTHOR (s): Energy, U.S. Department of } \\
\hline \multicolumn{5}{|c|}{ AUTHOR AFFILIATION: U.S. Department of Energy } \\
\hline \multicolumn{5}{|c|}{ TITLE: $\quad$ Satellite Power System (SPS) FY 79 Program Summary } \\
\hline DATE : & anuary 1980 & VOLUMES: & PAGES: 208 & REFERENCES: None \\
\hline \multicolumn{4}{|c|}{ ACQUISITION NO: DOE/ER-0037 } & SOURCE: \\
\hline \multicolumn{4}{|c|}{$\begin{aligned} \text { KEY WORDS: } & \text { Atmospheric Effects } \\
& \text { Comparative Assessment } \\
& \text { Environmental Assessment }\end{aligned}$} & $\begin{array}{l}\text { Reference System } \\
\text { Societal Assessment } \\
\text { Systems Definition }\end{array}$ \\
\hline
\end{tabular}


AUTHOR(S): Energy Research and Development Administration National Aeronautics and Space Administration

AUTHOR AFFILIATION: Energy Research and Development Administration National Aeronautics and Space Administration

TITLE: SPS Concept Development and Evaluation Program Plan

\begin{tabular}{|l|l|l|l|}
\hline DATE: May 1977 & VOLUMES: 2 & PAGES: 209 & REFERENCES: 28 \\
\hline ACQUISITION NO: $:$ NMI 1052.200 & & SOURCE: \\
\hline
\end{tabular}

KEY WORDS: Comparative Assessment Environmental Assessment Program Objectives
Socio-Economic Space Technology Systems Definition

SUBJECT: PROGRAM PLANS

SPSPO INDEX NO: PP-8

$\operatorname{AUTHOR}(s)$ : Logsdon, J. M.

AUTHOR AFFILIATION: George Washington University

TITLE: The Apol10 Decision and Its Lessons for Policy-Makers

\begin{tabular}{|l|l|}
\hline DATE: January 1970 & VOLUMES: \\
\hline ACQUISITION NO: Grant. 09-010-030 & \\
KEY WORDS: & $\begin{array}{l}\text { Decision Process } \\
\text { Lessons Learned } \\
\text { Space Transportation }\end{array}$
\end{tabular}




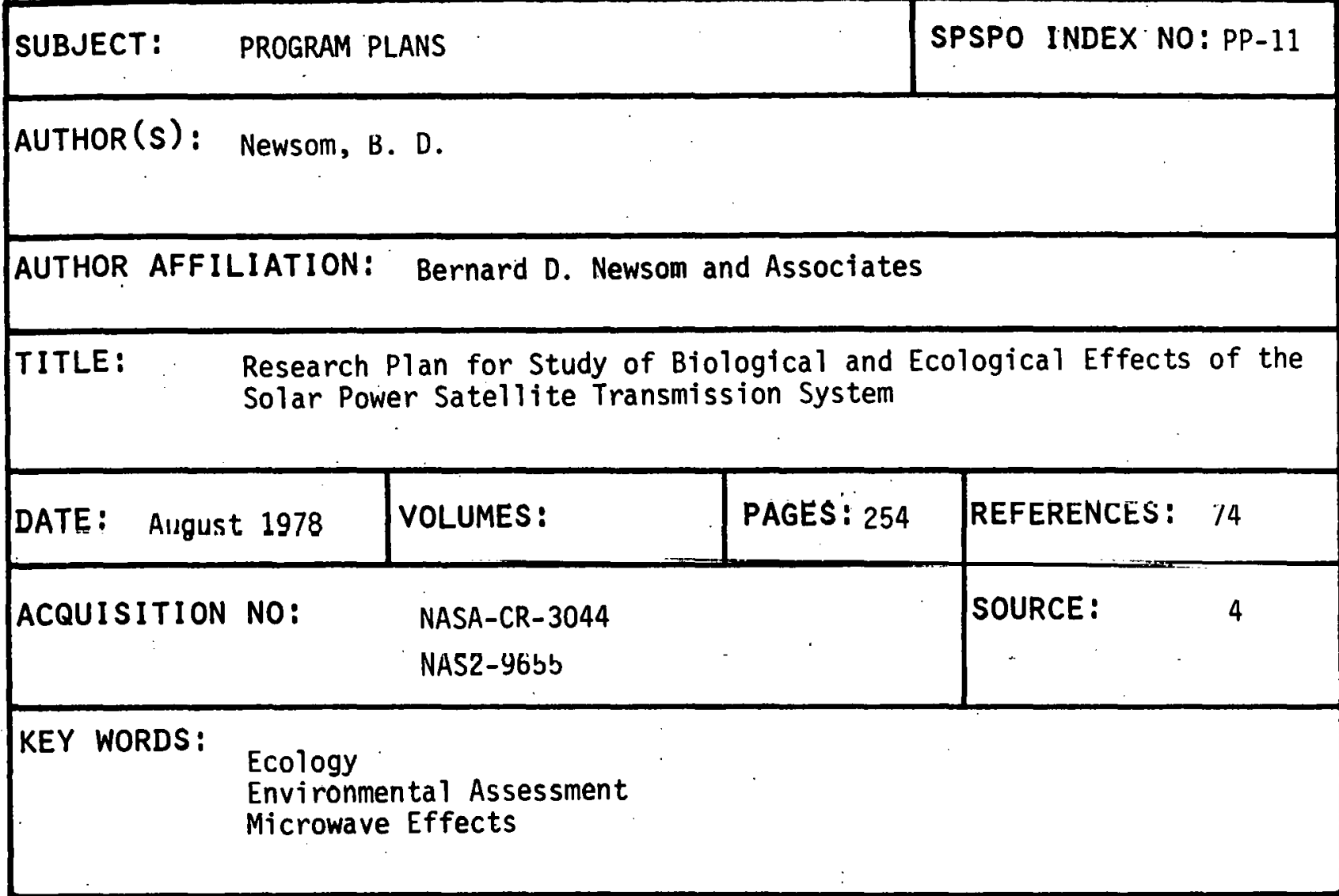

\begin{tabular}{|c|c|c|c|}
\hline \multicolumn{3}{|c|}{ PROGRAM PLANS } & PSPO INDEX NO: PP-7 \\
\hline \multicolumn{4}{|c|}{ AUTHOR(s): Universities Space Research Association } \\
\hline \multicolumn{4}{|c|}{ AUTHOR AFFILIATION: Universities Space Research Association } \\
\hline \multicolumn{4}{|c|}{ TITLE: $\quad$ Report of the Solar-Power Satellite Task Group } \\
\hline DATE: March 1 & VOLUMES: & PAGES: 25 & REFERENCES: None \\
\hline \multicolumn{3}{|c|}{ ACQUISITION NO: By TItle } & SOURCE: \\
\hline \multicolumn{3}{|c|}{$\begin{aligned} \text { KEY WORDS : } & \text { Atmospheric Effects } \\
& \text { Environmental Assessment } \\
& \text { Health Effects }\end{aligned}$} & $\begin{array}{l}\text { Societal Assessment } \\
\text { System Operations } \\
\text { Technology Requirements }\end{array}$ \\
\hline
\end{tabular}




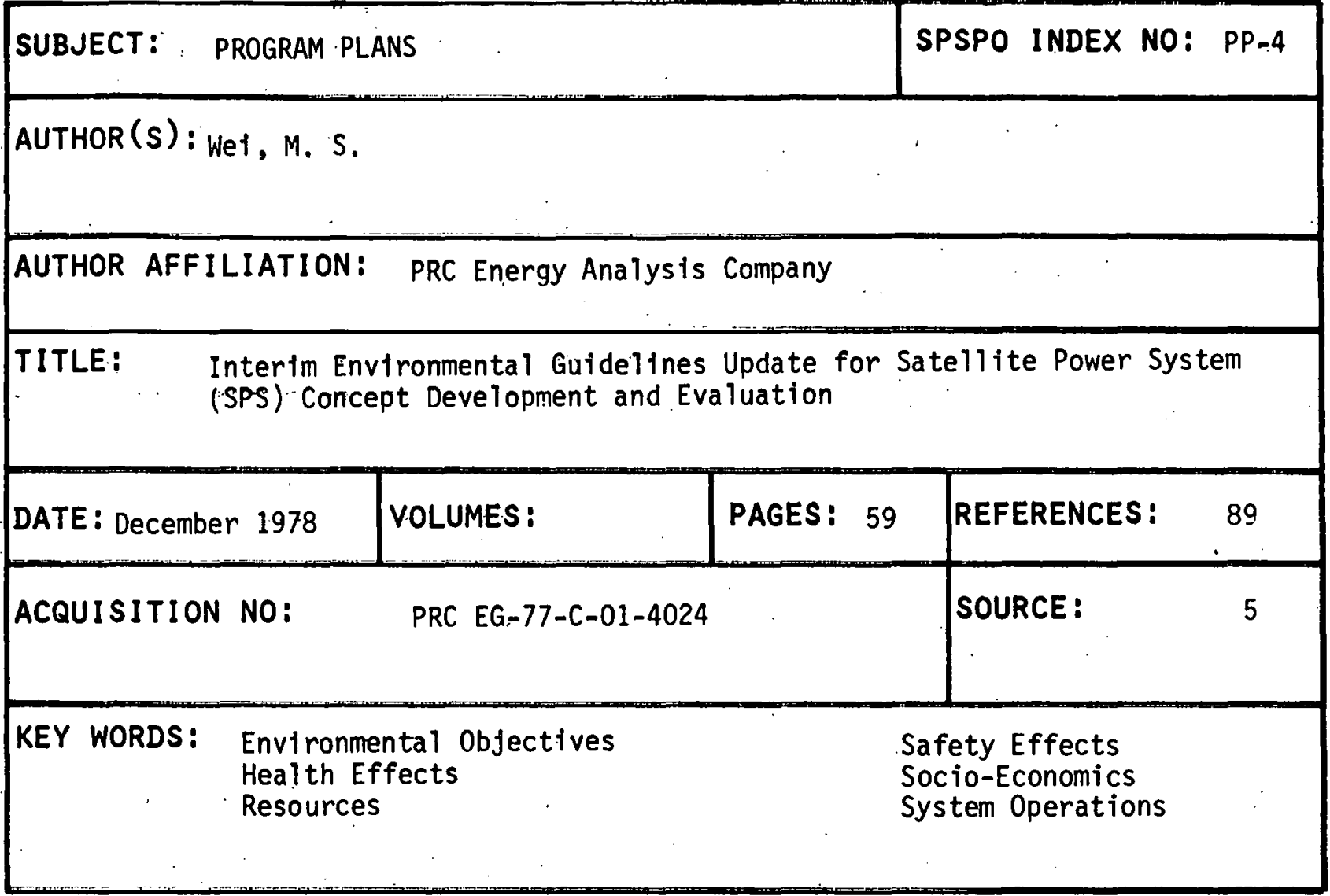




\section{THIS PAGE}

\section{WAS INTENTIONALLY \\ LEFT BLANK}




\section{POWER GENERATION AND TRANSMISSION}

\section{(CATEGORY PT)}

Research, development, experimentation and evaluation of engineering concepts and preliminary design of SPS power generation and transmission equipment is included in this category. A11 aspects of the transmitting system are included, such as the electrical characteristics of the space transmitting antenna, but the receiving. system (rectenna). is excluded as a principal topic. Environmental effects are described in some documents.

KEY WORDS :

\section{Antenna}

Atmospheric Effects

Broadcast

Cathode Life

Computer Prediction

Design

Economics

Efficiency

Experiments

Frequency Selection

Gain Degradation

Hardware

Harmonics

High Voltage.

Interference

Laser Alternatives

Laser Costs

Laser Power

Laser Safety

Magnetron Performance

Mechanical System

Microwave

Noise Characteristics
PAGE $\cdot$ NUMBER :

$123,124,125,126,127,128,129,130,131$, $132,133,134,135,136$

$123,133,134,136,138$

131

127

132

$123,124,125,126,127,128,120,130,131$, $133,135,136$

$121,122,125,127,137$

$121,123,124,125,126,127,128,131,133$,

136,138

$124,125,128,129,130,131,132$

$125 ; 131,138$

132

$126,134,136$

128,129

130,132

137

$123,133,135,136,138$

133,138

$121,122,123,130,133,135,136,138$

133

127,128

$123,126,134$

$123, .124,126,127,131,132,135,137$

127,128 
(Power Generation and Transmission Category PT continued)

KEY WORDS :

Phase Control

Phase Error

Photokiystron

Photovoltaics

Pointing Error

Power Amplifier

Power Conversion

Power Density

Propagation

Radiation Statistics

Receiver System

Reliability

Retrodirective Array

Solar Conversion

Solid State

Space Reflector

Technology

Thermal Systems

Waste Heat

RELATED CATEGORIES:

Atmospheric Effects (AE)

Electromagnetic Compatibility (EC)

Environmental Effects (EE)

Pliuluvolta1cs (PV)

Systemis Analyses (SA)

Systems Engineering (SE)

Space Utilization (SU)
PAGE NUMBER:

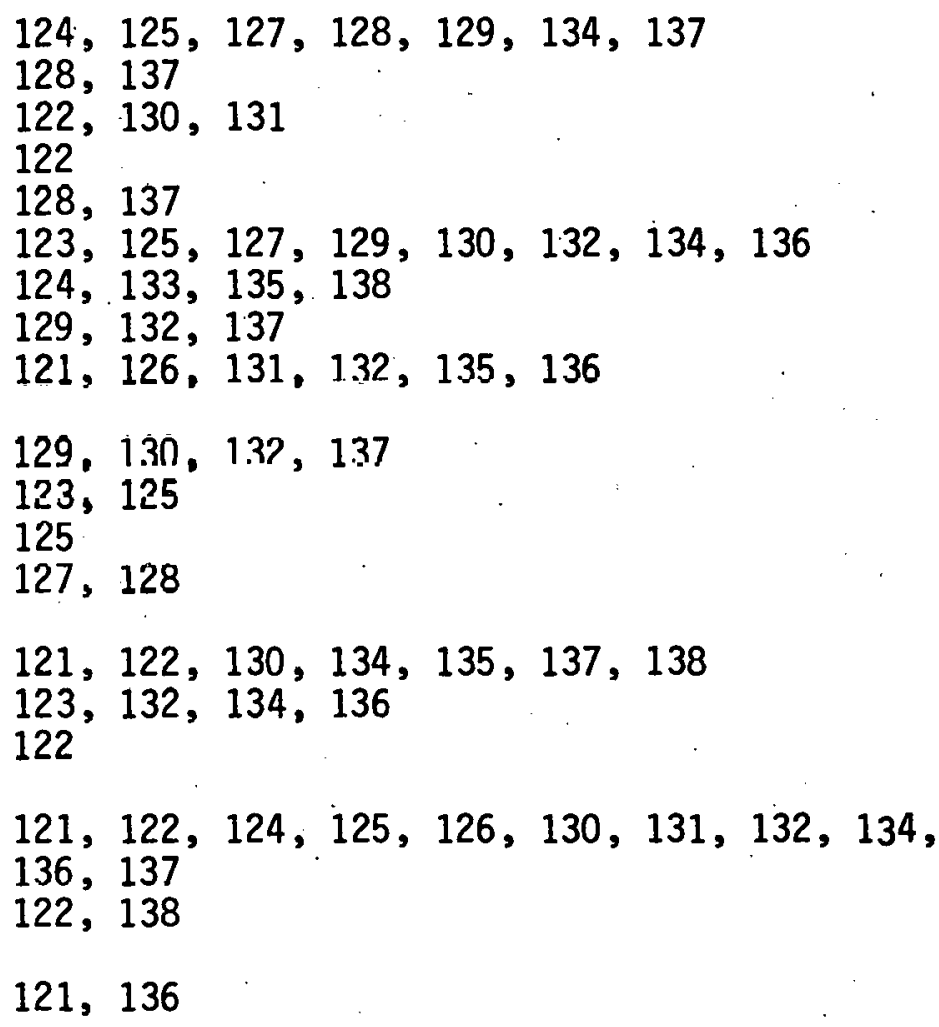

121,136 
AUTHOR (S): Bain, C.N.

AUTHOR AFFILIATION: PRC Energy Analysis Company

TITLE: Potential of Laser for SPS Power Transmission

\begin{tabular}{|c|c|c|c|c|}
\hline DATE: $\quad$ October 1978 & VOLUMES : & PAGES: 97 & REFERENCES : & 80 \\
\hline ACQUISITION NO: & $H C P / R-40 \angle 4-0 ̄ 7$ & & SOURCE: & 5 \\
\hline
\end{tabular}

KEY WORDS: Economics

Efficiency,

Laser Power
Propagation

Solar Conversion

Techno logy

SUBJECT:

POWER GENERATION AND TRANSMISSION

SPSPO INDEX NO: PT-6

AUTHOR(S): Bain, C. N.

AUTHOR AFFILIATION: PRC Energy Analys is Company

TITLE: Power from Space by Laser

\begin{tabular}{|l|l|l|l|}
\hline DATE: March 1979 & VOLUMES: & PAGES: 13 & $\begin{array}{l}\text { REFERENCES: } \\
\text { Bibliography }\end{array}$ \\
\hline $\begin{array}{l}\text { ACQUISITION NO: Astronautics and Aeronautics } \\
\text { Vol. } 17, \text { No. 3 } \\
\text { pp. 28-40 }\end{array}$ & SOURCE: \\
\hline
\end{tabular}

KEY WORDS: Economics

Efficiency

Technology

Laser Power

Waste Heat 


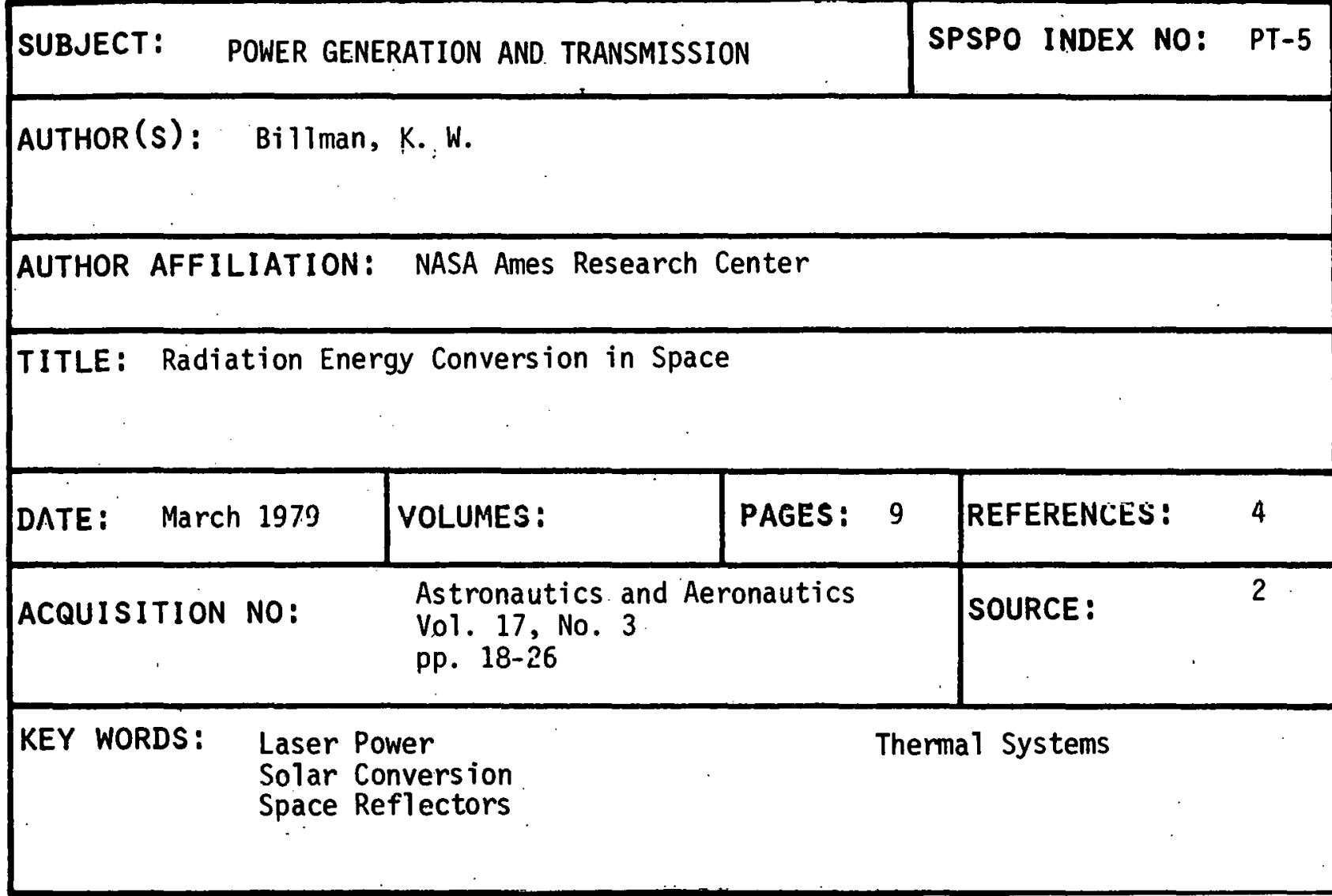

\section{SUBJECT:}

POWER GENERATION AND TRANSMISSION

SPSPO INDEX NO: PT-7

AUTHOR(s): Boeing Aerospace Company

AUTHOR AFFILIATION: Boeing Aerospace Company

TITLE: Systems Definition Space Based Power Conversion Systems

\begin{tabular}{|c|c|c|c|c|c|c|}
\hline DATE: March & 1977 & VOLUMES: & 2 & PAGES : 117 & REFERENCES : & None \\
\hline \multicolumn{5}{|c|}{ 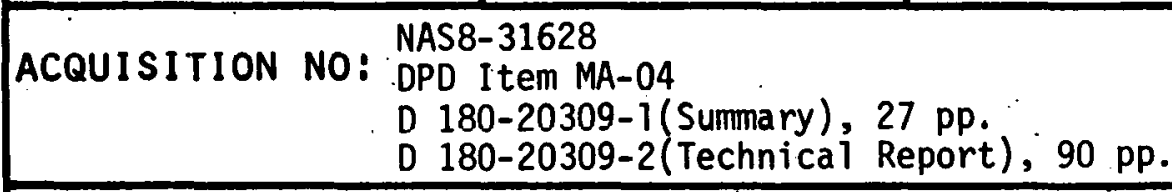 } & SOURCE: & 4 \\
\hline KEY WORDS: & \multicolumn{4}{|c|}{$\begin{array}{l}\text { Economics } \\
\text { Photovoltaics } \\
\text { Solar Conversion }\end{array}$} & \multicolumn{2}{|l|}{$\begin{array}{l}\text { Technology } \\
\text { Therma1 Sys tems }\end{array}$} \\
\hline
\end{tabular}




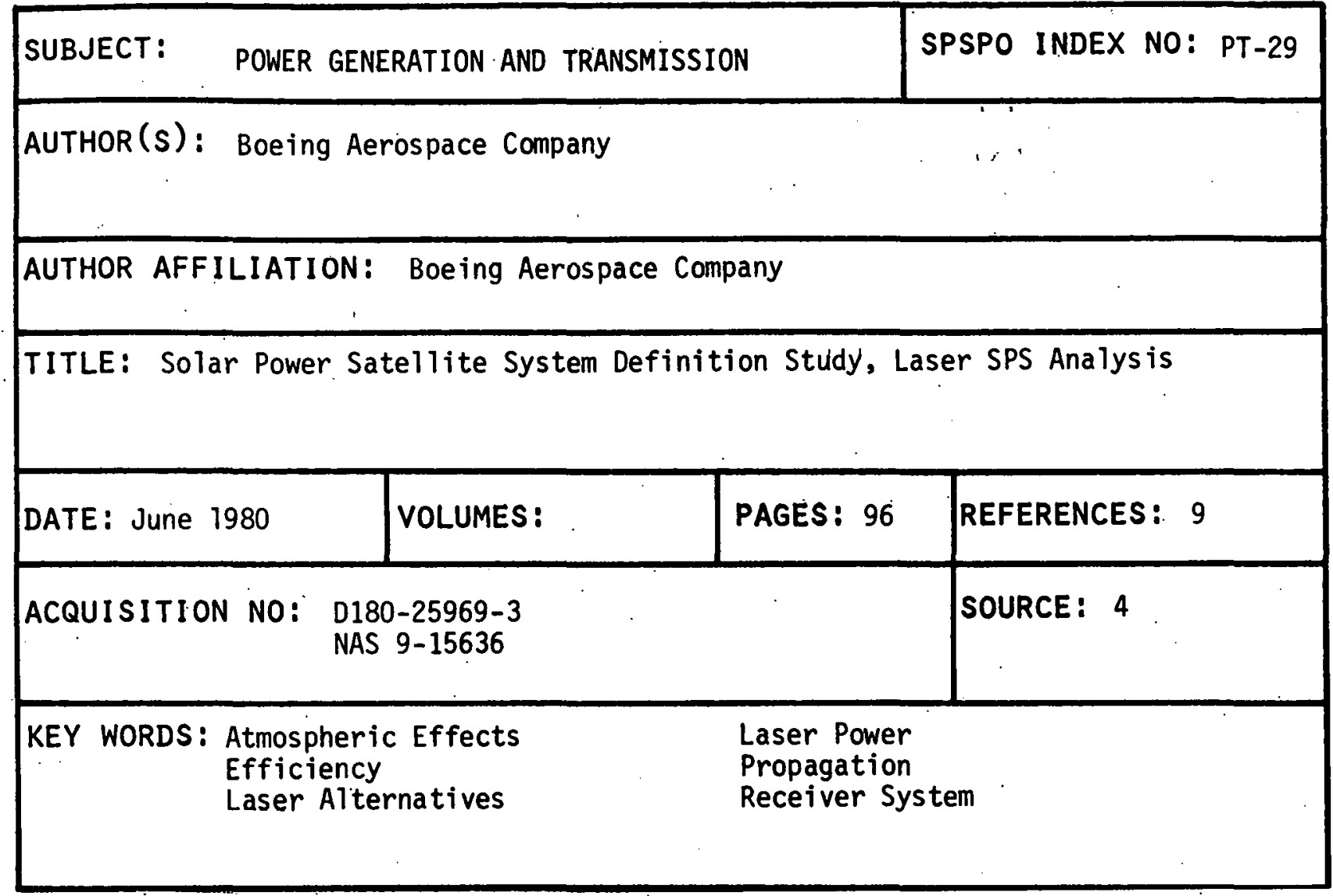

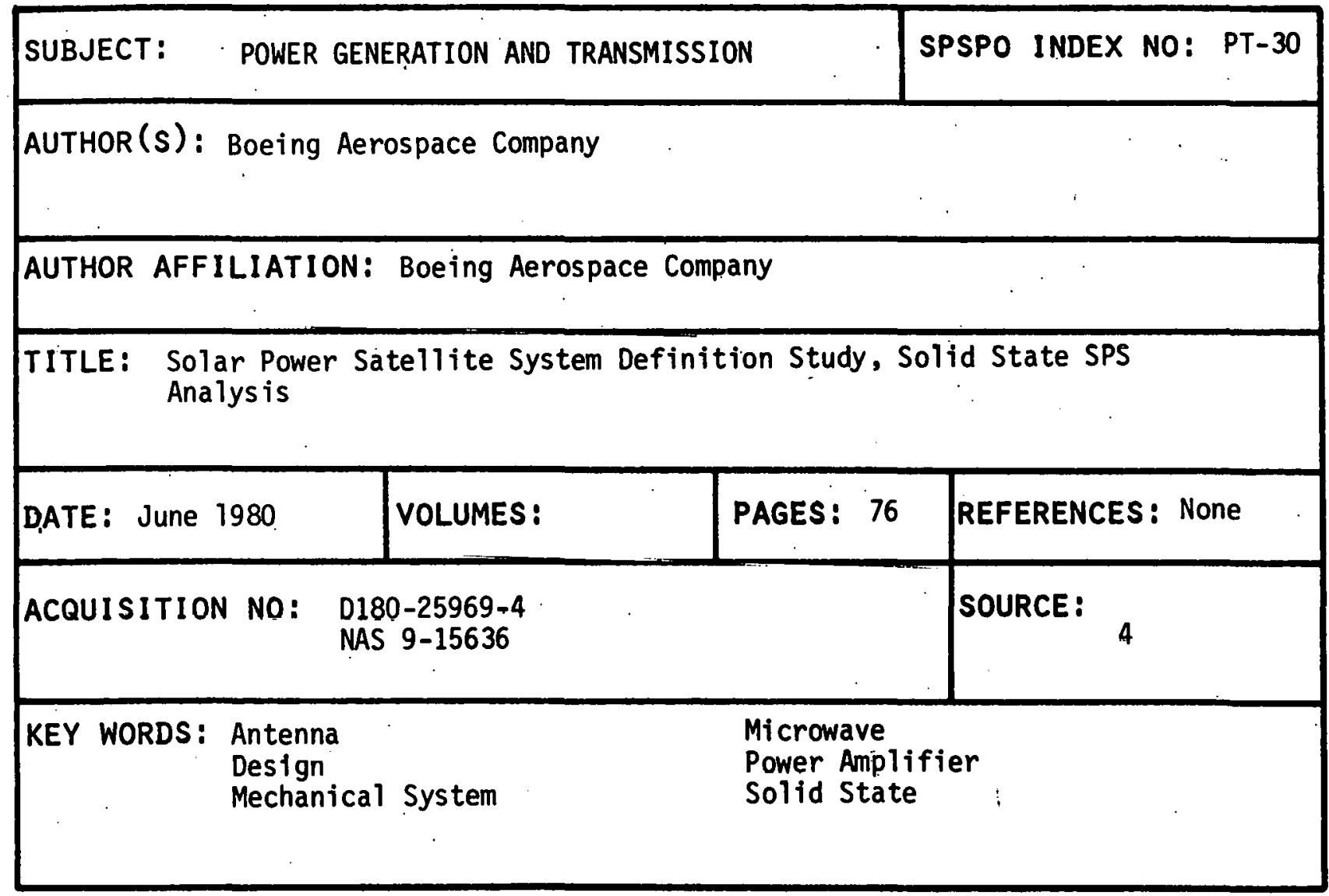


SUBJECT: 'POWER GENERATION AND TRANSMISSION

$\operatorname{AUTHOR}(s):$ Brown, W. C.

AUTHOR AFFILIATION: Raytheon Company

TITLE: $\quad$ The Technology and Application of Free-Space Power Transmission by Microwave Beam

\begin{tabular}{|l|l|l|l}
\hline DATE: January 1974 & VOLUMES: & PAGES: 15 & REFERENCE \\
\hline ACQUISITION NO: & $\begin{array}{l}\text { Proc. IEEE; Vol. 62, No. 1, } \\
\text { pp. 11-25 }\end{array}$ & SOURCE: \\
\hline KEY WORDS: & $\begin{array}{l}\text { Antenna } \\
\text { Design } \\
\text { Efficlency }\end{array}$ & $\begin{array}{l}\text { Microwave } \\
\text { Power Conversion } \\
\text { Technology }\end{array}$
\end{tabular}

SUBJECT: POWER GENERATION AND TRANSMISSION

AUTHOR (s): Brown, W. C.

Maynard, 0. E.

AUTHOR AFFILIATION: Raytheon Company

TITLE: $\quad$ The Adaptation of Free Space Power Transmission Technology to the SSPS Concept

\begin{tabular}{|c|c|c|c|c|c|c|}
\hline DAIE: April & 1975 & VOLUMES: & PAGES: & 11 & REFERFNC.ES : & 10 \\
\hline ACQUISITION & NO: & AIAA-75-642 & & & SOURCE: & 2 \\
\hline KEY WORDS : & $\begin{array}{l}\text { Effic } \\
\text { Exper } \\
\text { Micro }\end{array}$ & & & $\begin{array}{l}\text { Phi } \\
\text { Por } \\
\text { Ter }\end{array}$ & $\begin{array}{l}\text { e Control } \\
r \text { Conversion } \\
\text { nology }\end{array}$ & \\
\hline
\end{tabular}


$\operatorname{AUTHOR}(s):$ Brown, W. C.

AUTHOR AFFILIATION: Raytheon Company

TITLE: Technology Forecast for Free-Space Power Transmission by Microwave Beam

\begin{tabular}{|l|l|l|l|}
\hline DATE: June 1976 & VOLUMES: & PAGES: 41 & REFERENCES: None \\
\hline ACQUISITION NO: & PT-4808(21-4) & SOURCE: & 6 \\
\hline
\end{tabular}

KEY WORDS: $\begin{array}{ll}\text { Economics } \\ \text { Efficiency }\end{array}$

Frequency Selection

Phase Control

Reliability

Technology

SUBJECT: POWER GENERATION AND TRANSMISSION

SPSPO INDEX NO: PT-35

AUTHOR (s): Brown, W. C.

AUTHOR AFFILIATION: Raytheon Company

TITLE: Transmission of Power from Space to Earth

\begin{tabular}{|l|l|l|l|}
\hline DATE: August 1977 & VOLUMES: & PAGES: 11 & REFERENCES: 24 \\
\hline ACQUISITION NO: $\begin{array}{l}\text { By Title; New Options in Energy } \\
\text { Technology (San Francisco) }\end{array}$ & SOURCE: 6 \\
\hline
\end{tabular}

KEY WORDS: Antenna

Design

Experiments

Efficiency

Power. Amplifier

Receiver System 
SUBJECT: POWER GENERATION AND TRANSMISSION

$\operatorname{AUTHOR}(s)$ : Brown, W. C.

AUTHOR AFFILIATION: Raytheon Company

TITLE: $\quad$ Electronic and Mechanical Improvment of the Receiving Terminal of a Free-Space Microwave Power Transmission System

\begin{tabular}{|l|l|l|l|}
\hline DATE: August 1977 & VOLUMES: 1 & PAGES: 148 & REFERENCES: 31 \\
\hline ACQUISITION NO: & $\begin{array}{l}\text { NASA CR-135194 } \\
\text { PT-4964 }\end{array}$ & 3OURCE : \\
\hline
\end{tabular}

KEY WORDS: Antenna

Design

Hardware

Efficiency

Mechanical System

Technology

SUBJECT: POWER GENERATION AND TRANSMISSION

SPSPO INDEX NO: PT-3

AUTHOR (s): Brown, W. C.

AUTHOR AFFILIATION: Raytheon Company

TITLE: A Profile of Poiwer Transmission by Microwaves

\begin{tabular}{|l|l|l|l|}
\hline DATE: May 1979 & VOLUMES: & PAGES: 6 & REFERENCES: \\
\hline ACQUISITION NO: & $\begin{array}{l}\text { Astronautics and Aeronautics } \\
\text { Vol. 17, No. } 5\end{array}$ & SOURCE: \\
\hline KEY WORDS: $\begin{array}{l}\text { Antenna } \\
\begin{array}{l}\text { Efficiency } \\
\text { Hardware }\end{array}\end{array}$ & $\begin{array}{l}\text { Mfcrowave } \\
\text { Propagation }\end{array}$
\end{tabular}


SUBJECT: POWER GENERATION AND TRANSMISSION

AUTHOR.(s): BroWn, W. C.

AUTHOR AFFILIATION: Raytheon Company

TITLE:

Solar Power Satellites: Microwaves Deliver the Power

\begin{tabular}{|l|l|l|l|}
\hline DATE: June 1979 & VOLUMES: & PAGES: 5 & REFERENCES: None \\
\hline ACQUISITION NO: 0018-9235/79/0600-0036 & SOURCE: & 6 \\
\hline KEY WORDS: $\begin{array}{l}\text { Antenna } \\
\text { Economics } \\
\text { Efficiency }\end{array}$ & $\begin{array}{l}\text { Phase Control } \\
\text { Power Amplifier } \\
\text { Retrodirective Array }\end{array}$ \\
\hline
\end{tabular}

SUBJECT: POWER GENERATION AND TRANSMISSION

SPSPO INDEX NO: PT-25

AUTHOR(s): Brown, W. C.

AUTHOR AFFILIATION: Raytheon Company.

TITLE: Microwave Beamed Power Technology Improvement

\begin{tabular}{|l|l|l|l|}
\hline DATE: May 1980 & VOLUMES: & PAGES: 135 & REFERENCES: None \\
\hline ACQUISITION NO: $\begin{array}{l}\text { PT-5613 } \\
\text { JPL Contract 955104 } \\
\text { NAS 7-100 }\end{array}$ & SOURCE: 4 \\
\hline KEY WORDS: $\begin{array}{l}\text { Cathode Life } \\
\text { Design } \\
\text { Efficiency }\end{array}$ & $\begin{array}{l}\text { Magnetron Performance } \\
\text { Microwave } \\
\text { Noice Characteristics }\end{array}$ \\
\hline
\end{tabular}




\section{SUBJECT: POWER GENERATION AND TRANSMISSION}

$\operatorname{AUTHOR}(s)$ : Brown, W. C.

AUTHOR AFFILIATION: Raytheon Company

TITLE: Satellite Power System (SPS) Magnetron Tube Assessment Study

\begin{tabular}{|c|c|c|c|c|c|}
\hline DATE: July & 1980 & VOLUMES: & PAGES: 195 & REFERENC & s: \\
\hline ACQUISITION & NO: & $\begin{array}{l}\text { PT-5653 } \\
\text { NAS 8-33157 }\end{array}$ & & SOURCE: & 4 \\
\hline KEY WORDS: & $\begin{array}{l}\text { Desig } \\
\text { Effic } \\
\text { Exper }\end{array}$ & $\begin{array}{l}\text { ency } \\
\text { ments }\end{array}$ & $\begin{array}{l}\text { Harmonics } \\
\text { Magnetron } \\
\text { Noise Chara }\end{array}$ & $\begin{array}{l}\text { formance } \\
\text { teristics }\end{array}$ & \\
\hline
\end{tabular}

SUBJECT: POWER GENERATION AND TRANSMISSION

$\operatorname{AUTHOR}(S)$ : Chernoff, R. C.

AUTHOR AFFILIATION: Jet Propulsion Laboratory

TITLE: Large Active Retrodirective Arrays for Space Applications

\begin{tabular}{|l|l|l|l|}
\hline DATE: January 1978 & VolUMES: & PAGES: 53 & REFERENCES: 14 \\
\hline ACQUISITION NO: $\begin{array}{l}\text { NAS7-100 } \\
\text { JPL 78-20 }\end{array}$ & SOURCE: i \\
\hline KEY WORDS: $\begin{array}{l}\text { Antenna } \\
\text { Phase Control } \\
\text { Phase Error }\end{array}$ & $\begin{array}{l}\text { Pointing Error } \\
\text { Retrodirective Array }\end{array}$ \\
\hline
\end{tabular}


SUBJECT: POWER GENERATION AND TRANSMISSION

AUTTHOR(S): Dickinson, R: M.

AUTHOR AFFILIATION:

Jet Propulsion Laboratory

TITLE:

Microwave Power Transmitting Phased Array Antenna Research Project Summary Report

\begin{tabular}{|c|c|c|c|c|c|}
\hline \multicolumn{2}{|c|}{ DATE: December 1978} & VOLUMES: & PAGES: 74 & REFERENCES : & 9 \\
\hline ACQUISITION N & NO: & JPL-78-28 & & SOURCE : & 4 \\
\hline KEY WORDS: & $\begin{array}{l}\text { Antenn } \\
\text { Design } \\
\text { Experi }\end{array}$ & & & $\begin{array}{l}\text { ase Control } \\
\text { wer Amplifier } \\
\text { wer Density }\end{array}$ & \\
\hline
\end{tabular}

SUBJECT: POWER GENERATION AND TRANSMISSION

AUTHOR(S): Dickinson, R. M.

AUTHOR AFFILIATION: Jet Propulsion Laboratory.

TITLE: Beamed Microwave Power Transmitting and Receiving Subsystems Radiation Characteristics

DATE: June 1980 ' VOLUMES:

PAGES: 83 REFERENCES: 30

ACQUISITION NO: JPL 80-11

SOURCE : 4

KEY WORDS: Antenna

Design

Harmonics
Power Amplifier

Power Density

Radiation Statistics 
SUBJECT: POWER GENERATION AND TRANSMISSION

AUTHOR(s): Fitzsimmons, G. W.

Nalos, E. J.

AUTHOR AFFILIATION: Boeing Aerospace Company

TITLE: SPS Solid-State Antenna Power Combiner

DATE: February 1980

VOLUMES:

\begin{tabular}{l|l} 
PAGES: 99 & REFERENCES: 1
\end{tabular}

ACQUISITION NO: D 180-25895-1

SOURCE: $\quad 4$

NAS $9-15636 A$

\begin{tabular}{|c|c|c|}
\hline KEY WORDS: & $\begin{array}{l}\text { Antenna } \\
\text { Design } \\
\text { Efficiency }\end{array}$ & $\begin{array}{l}\text { Power Ampl ifier } \\
\text { Radiation Statistics } \\
\text { Technology }\end{array}$ \\
\hline
\end{tabular}

SUBJECT: POWER GENERATION AND TRANSMISSION

SPSPO INDEX NO: PT-2

AUTHOR (s): Freeman, J. W.

Colson, W. B.

Simons, $S$.

AUTHOR AFFILIATION: Rice University

TITLE: New Methods for the Conversion of Solar Energy to Radio Frequency and Laser Power

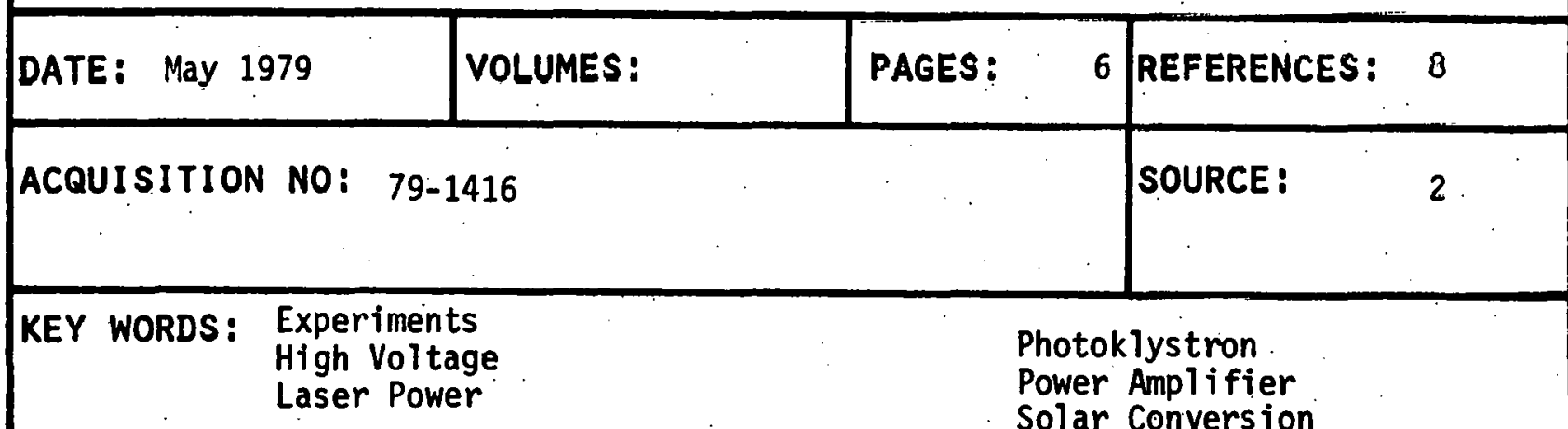




$\begin{array}{ll}\text { AUTHOR (S): } \begin{array}{l}\text { Freeman, J.W. } \\ \text { Simons, S. } \\ \text { Colson, W. B. }\end{array} & \begin{array}{l}\text { Brotzen, F.R. } \\ \text { Hester, J. }\end{array}\end{array}$

AUTHOR AFFILIATION: Rice University

TITLE: The Photoklystron

\begin{tabular}{|l|l|l|l|}
\hline DATE: 1980 & VOLUMES: & P,AGES: 10 & REFERENCES: 10 \\
\hline ACQUISITION NO: $\begin{array}{l}\text { 0191-9067/80/010145-10 } \\
\text { Space Solar Power Review, Vol. 1, } \\
\text { pp. 145-154 }\end{array}$ & SOURCE: 33 \\
\hline
\end{tabular}

KEY WORDS: Design Microwave

Experiments Photokiystron

Frequency Selection Technology

SUBJECT: POWER GENERATION AND TRANSMISSION

SPSPO INDEX NO:

PT-4

$\begin{array}{ll}\text { AUTHOR (s): } & \text { Fordyce, S. W. (1) } \\ \text { Brown, W. C. (2) }\end{array}$

AUTHOR AFFILIATION: (1) National Aeronautics and Space Administration

(2) Raytheon Company

TITLE: Applications of Free-Space Microwave Power Transmission

\begin{tabular}{|l|l|l|l|}
\hline DATE: September 1979 & VOLUMES: & PAGES: 6 & REFERENCES: \\
\hline ACQUISITION NO: $\begin{array}{l}\text { Astronautics and Apronautics } \\
\text { Vol. } 17, \text { No. } 9 \\
\text { pp. } 54-61\end{array}$ & SOURCE: \\
\hline
\end{tabular}

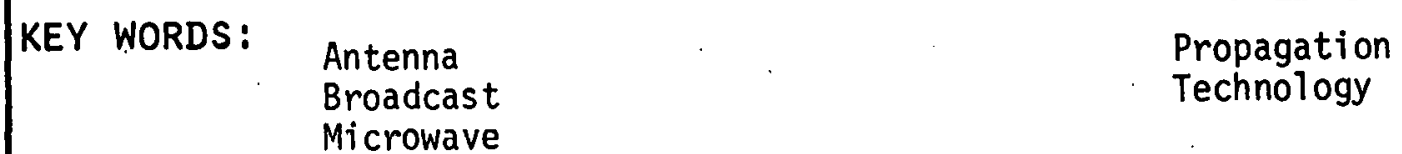


SUBJECT: POWER GENERATION AND TRANSMISSION

AUTHOR(s): Gibson, M. H.

Pollacsek, $M$.

Strijk, $S$.

AUTHOR AFFILIATION:

European Space Agency

TITLE: Further Investigation of RF Gain Degradation in High Power Microwave Transistors under Multicarrier Operation

DATE: 1978

VOLUMES :

\begin{tabular}{l|l} 
PAGES: 7 & REFERENCES: 1
\end{tabular}

ACQUISITION NO: ESA Journal 78/3, Vol. 2

pp. 207-213

SOURCE:

KEY WORDS: Computer Prediction

Experiments

Gain Degradation

High Voltage

Power Amplifier

Solid State

SUBJECT: . POWER GENERATION AND TRANSMISSION

SPSPO INDEX NO: PT-34

$$
\begin{aligned}
\text { AUTHOR (S): } & \text { Kozakoff, D. J. } \\
& \text { Schuchardt, J. M. }
\end{aligned}
$$

AUTHOR AFFILIATION: Georgia.Institute of Technology

TITLE: High Accuracy Radiation Efficiency Measurement Techniques

\begin{tabular}{|l|l|l|l|}
\hline DATE: January 1981 & VOLUMES: & PAGES: 82. & REFERENCES: 17 \\
\hline ACQUISITION NO: $\begin{array}{l}\text { NASA CR 3372 } \\
\text { NAS 8-33605 }\end{array}$ & SOURCE: 4 \\
\hline KEY WORDS: $\begin{array}{l}\text { Antenna } \\
\text { Microwave } \\
\text { Power Density }\end{array}$ & $\begin{array}{l}\text { Propagation } \\
\text { Radiation Statistics } \\
\text { Technology }\end{array}$ \\
\hline
\end{tabular}




\section{SUBJECT :}

POWER GENERATION AND TRANSMISSION

AUTHOR(s): Lockheed Missiles and Space Company

AUTHOR AFFILIATION: Lockheed Missiles and Space Company

TITLE: Laser Power Conversion System Analysis

\begin{tabular}{|l|l|l|l|}
\hline DATE: September 1978 & VOLUMES: 2 & PAGES: 205 & REFERENCES: 56 \\
\hline ACQUISITION NO: & NASA-CR-159523 & 4 \\
\hline KEY WORDS: & $\begin{array}{l}\text { Antenna } \\
\text { Atmospheric Effects } \\
\text { Laser Cost }\end{array}$ & $\begin{array}{l}\text { Laser Power } \\
\text { Laser Safety } \\
\text { Power Conversion }\end{array}$ \\
\hline
\end{tabular}

SUBJECT: POWER GENERATION AND TRANSMISSION

$\operatorname{AUTHOR}(S)$ : Mathematical Sciences Northwest, Inc.

AUTHOR AFFILIATION: Mathematical Sciences Northwest, Inc.

TITLE: Study, Optimization, and Design of a Laser Heat Engine

\begin{tabular}{|c|c|c|c|}
\hline DATE : January 1978 & VOLUMES: & PAGES : 158 & REFERENCES : 74 \\
\hline \multicolumn{3}{|c|}{ ACQUISITION NO: MSNW $78-1082-1$} & SOURCE : 4 \\
\hline $\begin{aligned} \text { KEY WORDS: } & \text { Design } \\
& \text { Efficiency } \\
& \text { Laser Alt }\end{aligned}$ & rnatives & Laser Power & \\
\hline
\end{tabular}




\begin{tabular}{|c|c|c|}
\hline $\operatorname{AUTHOR}(s):$ & $\begin{array}{l}\text { Maynard, 0. E. (1) } \\
\text { Brown, W. C. } \\
\text { Edwards, A. (1) }\end{array}$ & $\begin{array}{l}\text { Meltz, G. } \\
\text { Haley, J. T. (1) } \\
\text { Howeli, J. M. (1) }\end{array}$ \\
\hline
\end{tabular}

AUTHOR AFFILIATION: (1) Raytheon Company

(2) Grumman Aerospace Corporation

TITLE: Microwave Power Transmission System Studies

DATE : 'December 1975

VOLUMES: 4

PAGES: 771 REFERENCES: 42

Voi. 1 - Summary (43 pp.)

ACQUISITION NO: Vol. 2 - Analys is (261 pp.)

NASA CR-134886

Vol. 3 - Mechanical Sys tems

ER $74-4368$

Vol. 4 - Antennas (274 pp.)

KEY WORDS: Antenna

Mechanical System

Phase Control

SOURCE :

(233 pp.) 4

Solar Conversion

Technology

SUBJECT: ' POWER GENERATION AND TRANSMISSION

SPSPO INDEX NO: PT-33

AUTHOR(\$): Maynard, 0. E.

AUTHOR AFFILIATION: Raytheon Company

TITLE: Solid State SPS Microwave Generation and Transmission Study

DATE: Nưvember 1980

VOLUMES : 2

PAGES: 343

REFERENCES: 20

ACQUISITION NO:

NASA CR 3338 and 3339

NAS 8-33157

SOURCE : 4

V.l, Final Report, $230 \mathrm{pp}$.

V.2, Appendix, $113 \mathrm{pp}$.

KEY WORDS: Antenna

Atmospheric Effects

Design

Hardware

Power Amplifier

Solid State 
SUBJECT:

POWER GENERATION AND TRANSMISSION

AUTHOR (S): Nalos, E. J.

AUTHOR AFFILIATION: Boeing Aerospace Company

TITLE: : New Developments in Electromagnetic Energy Beaming

\begin{tabular}{|l|l|}
\hline DATE: March 1978 & VOLUMES: \\
\hline
\end{tabular}

ACQUISITION NO: $\quad 0018-9219 / 78 / 0300-0276$

\begin{tabular}{l|l} 
PAGES: 14 & REFERENCES: 20
\end{tabular}

SOURCE： 6

KEY WORDS: Antenna

Design

Laser Power

Microwave

Phase Control

Power Amplifier

\begin{tabular}{|c|c|c|c|}
\hline \multicolumn{3}{|c|}{ SUBJECT: POWER GENERATION AND TRANSMISSION } & PSPO INDEX NO: PT-23 \\
\hline $\operatorname{AUTHOR}(\mathrm{S})$ : & \multicolumn{2}{|l|}{$\begin{array}{l}\text { Rather, J.D. G. } \\
\text { Gerry, E. T. } \\
\text { Zeiders, G. W. }\end{array}$} & \\
\hline \multicolumn{4}{|c|}{ AUTHOR AFFILIATION: W. J.Schafer Associates, Inc. } \\
\hline \multicolumn{4}{|c|}{$\begin{array}{l}\text { TITLE: } \begin{array}{l}\text { Investigation of Possibilities for Solar Powered High Energy } \\
\text { Lasers in Space }\end{array}\end{array}$} \\
\hline DATE: May 19 & VOLUMES: & PAGES: 47 & REFERENCES : 15 \\
\hline \multicolumn{3}{|c|}{ ACQUISITION NO: $\begin{array}{l}77 S R-V A-U 3 \\
\text { NASW-3048 }\end{array}$} & SOURCE : \\
\hline KEY WORDS : & \multicolumn{2}{|l|}{$\begin{array}{l}\text { Laser Alternatives } \\
\text { iaser Power } \\
\text { Power Conversion }\end{array}$} & \\
\hline
\end{tabular}


SUBJECT: POWER GENERATION AND TRANSMISSION

$\operatorname{AUTHOR}(S): \operatorname{Reilly,~J.P.~}$

AUTHOR AFFILIATION: W. J. Schaffer Associates, Inc.

TITLE: High Power Lasers

DATE : June-July 1978

VOLUMES:

PAGES: 6

REFERENCES: 6

ACQUISITION NO: By Title, Technology Review; pp 58-63

SOURCE : 29

KEY WORDS: Atmospheric Effects

Laser Power

Propagation

Technology

Waste Heat

SUBJECT: POWER GENERATION AND TRANSMISSION

SPSPO INDEX NO: PT-32

AUTHOR(S): Rockwell International.

AUTHOR AFFILIATION: Rockwell International

TITLE: Satellite Power System (SPS) Concept Definition Study, Solid State Amplifier Investigation

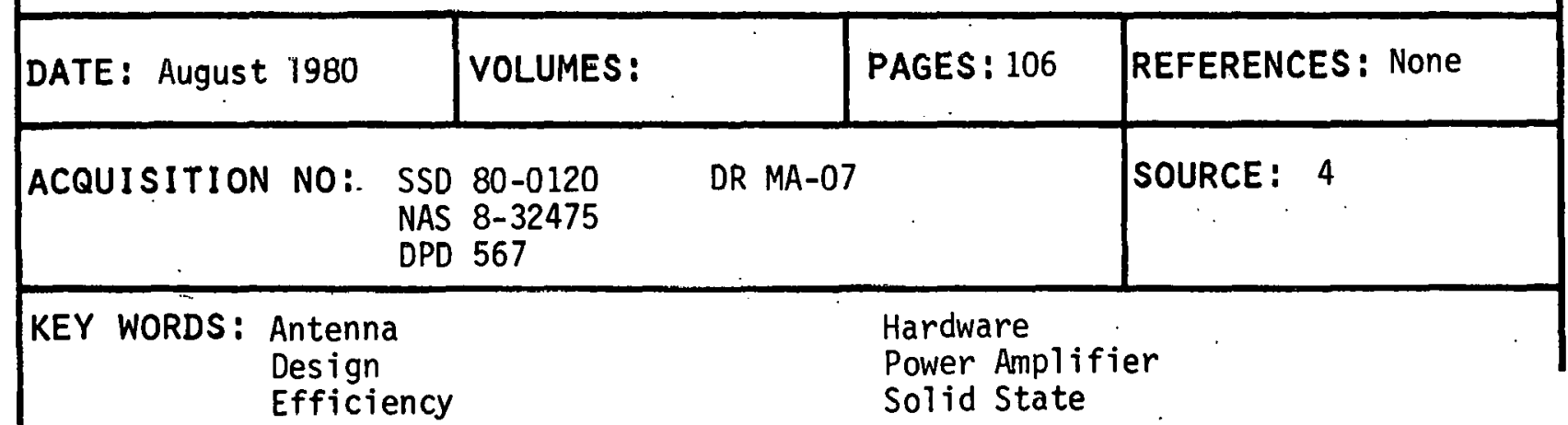




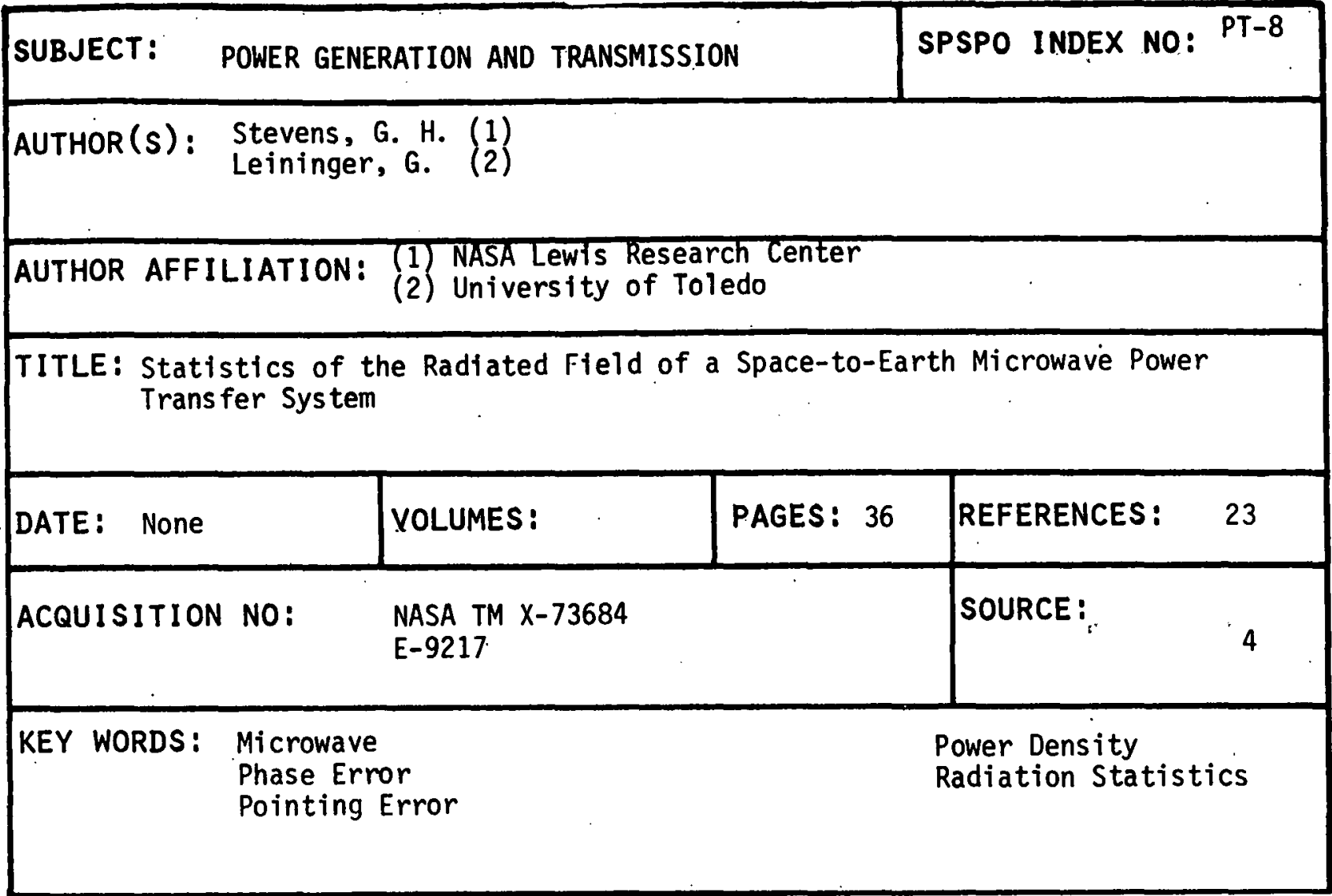

\section{\begin{tabular}{|ll}
\hline SUBJECT: & POWER GENERATION \\
\hline AUTHOR (S) & $\begin{array}{l}\text { Stevens, G. H. } \\
\text { Schuh, R. }\end{array}$
\end{tabular}}

AUTHOR AFFILIATION: NASA Lewis Research Center

TITLE: $\quad$ Space-to-Larth Power Transmission System

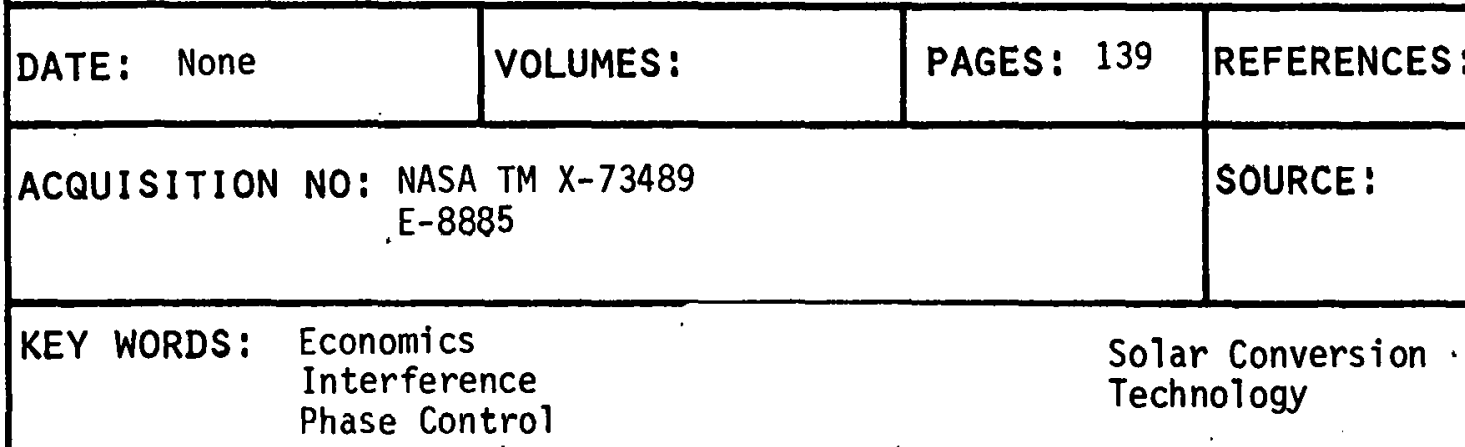




\begin{tabular}{|c|c|c|}
\hline SUBJECT: & POWER GENERATION AND & TRANSMISSION \\
\hline AUTHOR (S): & $\begin{array}{l}\text { Taussig, R. } \\
\text { Bruzzone, C. } \\
\text { Quimbly, D. } \\
\text { Nelson, L. }\end{array}$ & $\begin{array}{l}\text { Christiansen, W. } \\
\text { - Neice, S. } \\
\text { Cassady, P. } \\
\text { Pindroh, A. }\end{array}$ \\
\hline
\end{tabular}

AUTHOR AFFILIATION: Mathematical Sciences Northwest, Inc.

TITLE: . Design Investigation of Solar Powered Lasers for Space Applications

\begin{tabular}{|c|c|c|c|c|c|}
\hline DATE: May 19 & 979 & VOLUMES : & 175 & REFERENCES: & $82^{\prime}$ \\
\hline ACQUISITION. & NO: & \multicolumn{2}{|c|}{ MSNH-79-1087/1090-1 } & SOURCE : & 4 \\
\hline KEY WORDS : & \multicolumn{3}{|c|}{$\begin{array}{l}\text { Laser Alternatives } \\
\text { Laser Costs } \\
\text { Laser Power }\end{array}$} & \multicolumn{2}{|l|}{$\begin{array}{l}\text { Power Conversion } \\
\text { Solar Conversion } \\
\text { Therma } 1 \text { Systems }\end{array}$} \\
\hline
\end{tabular}

SUBJECT: - POWER GENERATION AND TRANSMISSION SPSPO INDEX NO: PT-24

AUTHOR(s): Walbridge, E. W.

AUTHOR AFFILIATION: Argonne National Laboratory

TITLE: Laser Satellite Power Systems

\begin{tabular}{|c|c|c|c|c|}
\hline DATE: January & 1980 & VOLUMES: & PAGES: 77 & REFERENCES : 39 \\
\hline \multicolumn{4}{|c|}{ ACQUISITION NO: ANL/ES-92 } & SOURCE : 5 \\
\hline KEY WORDS: & $\begin{array}{l}\text { Atmosp } \\
\text { Effici } \\
\text { Freque }\end{array}$ & $\begin{array}{l}\text { C Effects } \\
\text { Selection }\end{array}$ & $\begin{array}{l}\text { Laser Aiter } \\
\text { Laser Power }\end{array}$ & ives \\
\hline
\end{tabular}




\section{RESOURCES AND MATERIALS}

(CATEGORY RM)

Resources and materials which would be required to fully develop SPS, their source throughout the world and knowledge about current production and likely: needs by other ventures are described in documents in this category. Processes for evaluating resource and material needs also are included.

KEY WORDS:

Assessment

Cell Design

Commercial Market

Design Characterizations

Economics

Efficiency

Energy Payback

Energy Use

Federal Programs

Fuels

Inventory

Land

Land Survey

Manpower

Mapping

Materials Constraints

Materials Extraction

Materials Processing

Materials Requirements

Photovol taics

Problem Identification

Process Conversions

Production

Rectenna Sites

Sea Survey

Selection Methods

Water
PAGE NUMBER:

$141,143,144,145,146$

142

142

143

$142,144,146$

142

144

142

142

144

$141,143,145,146$

144

$141,142,143,145$

144

141,145

143,146

144,145

144,145

143

142

143

143

$141,145,146$

$141,142,144,145$

142

$141,142,143,145,146$

144 
(Resources and Materials

Category RM Continued)

RELATED CATEGORIES:

Energy Alternatives and Policy (EA)

Environmental Effects (EE)

Economics and Finances (EF)

Systems Analyses (SA)

Systems Engineering (SE)

Social Issues (SI) 


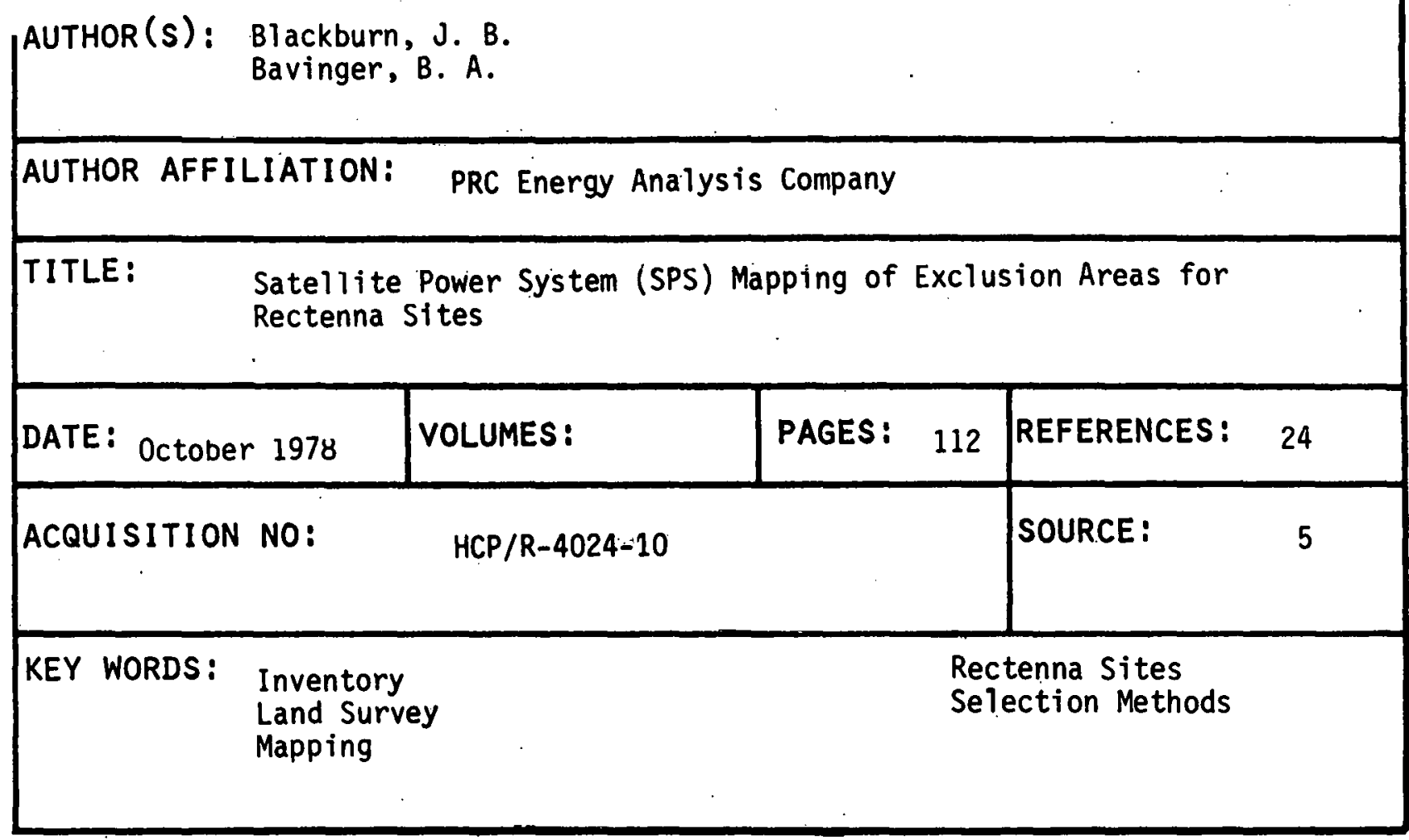

SUBJECT: RESOURCES AND MATERIALS

AUTHOR AFFILIATION: Battelle Columbus Laboratories

TITLE: A Methodology for Assessing Systems Materials Requirements

\begin{tabular}{|l|l|l|l|}
\hline DATE: January 1980 & VOLUMES: & PAGES: 40 & REFERENCES: \\
\hline ACQUISITION NO: & DOE/ER-0040 & SOURCE: \\
\hline
\end{tabular}

KEY WORDS: Assessment Methods

Inventory

Production 
SUBJECT : .. RESOURCES AND MATERIALS

AUTHOR (s): Eberhardt, A. W.

AUTHOR AFFILIATION: NASA Marshall Space Flight, Center

TITLE: Candidate Locations for SPS Rectifying Antennas

DATE: November 1977

VOLUMES:

PAGES : 8.3

REFERENCES:

15

ACQUISITION NO: NASA TM-78146

SOURCE :

4

KEY WORDS: Land Survey

Selection Methods

Rectenna Sites

Sea Survey

SUBJECT: RESOURCES AND MATERIALS

SPSPO INDEX NO: RM-12

AUTHOR (s): Ehrenreich, H.

Martin, J.H.

AUTHOR AFFILIATION: Harvard University

TITLE: Solar Photovoltaic Energy

DATE: September 1979

VOLUMES :

PAGES: 8

REFERENCES: $?$

ACQUISITION NO: By Title, Physics Today, pp. 25-32

SOURCE : 36

KEY WORDS: Cell Design

Commercial Market

Economics

Efficiency

Federal Programs

Photovoltaics 


\section{SUBJECT: RESOURCES AND MATERIALS}

$\operatorname{AUTHOR}(S):$ Kotin, A. D.

AUTHOR AFFILIATION: Allan D. Kotin Economic Consultants

TITLE: Satellite Power System (SPS) Resource Requirements (Critical Materials, Energy and Land)

\begin{tabular}{|l|l|l|l|}
\hline DATE: October 1978 & VOLUMES: & PAGES: 128 & REFERENCES: 87 \\
\hline ACQUISITION NO: & HCP/R-4024-02 & SOURCE: \\
\hline KEY WORDS: & $\begin{array}{l}\text { Assessment Methods } \\
\text { Energy Use } \\
\text { Land Survey }\end{array}$ & $\begin{array}{l}\text { Materials Constraints } \\
\text { Material Requirements }\end{array}$ \\
\hline
\end{tabular}

\section{SUBJECT:}

RESOURCES AND MATERIALS

AUTHOR(S): Litchfield, J. W. Hartley, J. N. Watts, R. L. Gurwe11, W. E.

\section{AUTHOR AFFILIATION:}

Pactfic Northwest Laboratory

TITLE: A Methodology for Identifying Materials Constraints to Implementation of Solar Energy Technologies

\begin{tabular}{|l|l|lc|}
\hline DATE: JUIy 1978 & VOLUMES: & PAGES: 84 & REFERENCES: 36 \\
\hline ACQUISITION NO: & SNL-2711 & 13 \\
\hline KEY WORDS: & $\begin{array}{l}\text { Design Characterizations } \\
\text { Inventory } \\
\text { Problem Identification }\end{array}$ & $\begin{array}{l}\text { Process Conversion } \\
\text { Production } \\
\text { Selection Methods }\end{array}$ \\
\hline
\end{tabular}


SUBJECT: .RESOURCES AND MATERIALS:

$\operatorname{AUTHOR}(s): \begin{aligned} & \text { Livingston, F.R. } \\ & \text { Baker, L.R. }\end{aligned}$

Poon, P. T. Y.

Kuo, T. J.

AUTHOR AFFILIATION: Jet Propulsion Laboratory

TITLE:

Satellite Power System (SPS) Preliminary Resource Assessment

DATE: August 1978

VOLUMES:

PAGES:

81

REFERENCES :

22

ACQUISITION NO: $\quad 900-805$ (Rev. A)

SOURCE:

4

KEY WORDS: $\begin{array}{ll}\text { Energy Payback } \\ \text { Fuels }\end{array}$

Manpower

Land

Rectenna Sites

Water

SUBJECT: ' RESOURCES AND MATERIALS

SPSPO INDEX NO: RM-5

$\operatorname{AUTHOR}(s)$ : Reeber, R. R.

AUTHOR AFFILIATION:

U.S. Department of Energy

TITLE :

An Overview: The Materials/Energ.y Tradeoff

DATE: June 1978.

VOLUMES:

\begin{tabular}{ll|l}
\hline PAGES: ? & REFERENCES:
\end{tabular}

35

ACQUISITION NO:

By Title: National Symposium on

Critical and Strategic Materials

SOURCE:

KEY WORDS: Assessment Methods

Economics

Energy Payback

Materials Extraction

Materials Processing 
AUTHOR(s): Rice University

AUTHOR AFFILIATION: Rice University

TITLE: Satellite Power System (SPS) Rectenna Siting: Availability and Distribution of Nominaliy Eligible Sites

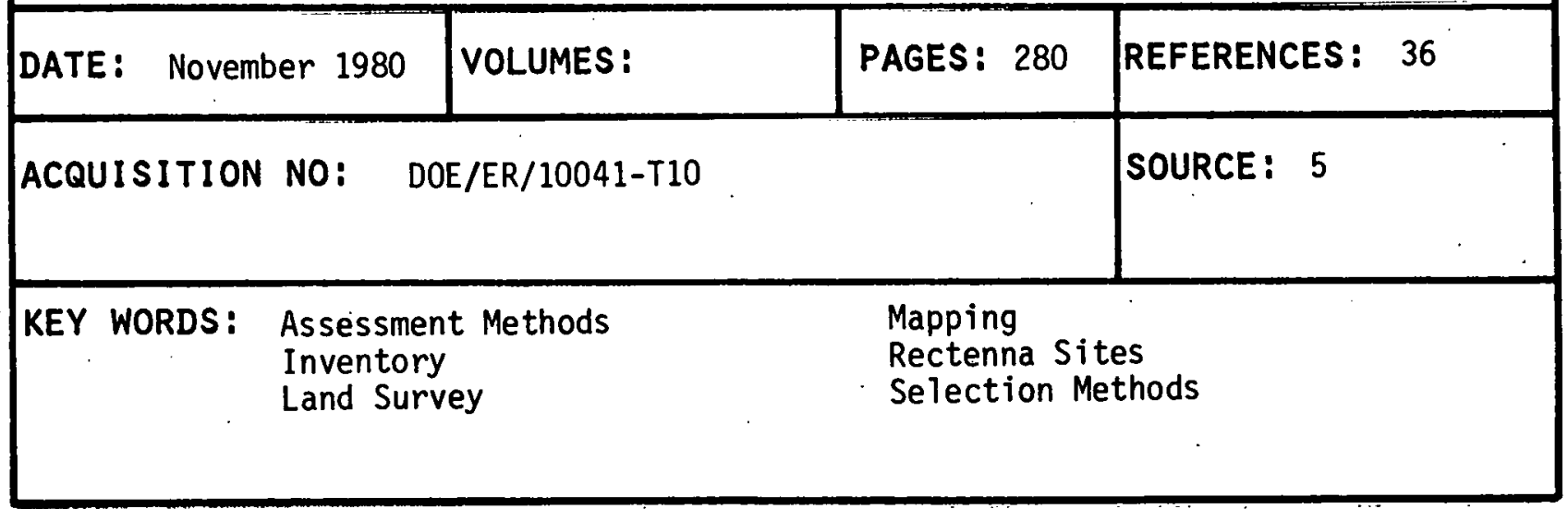

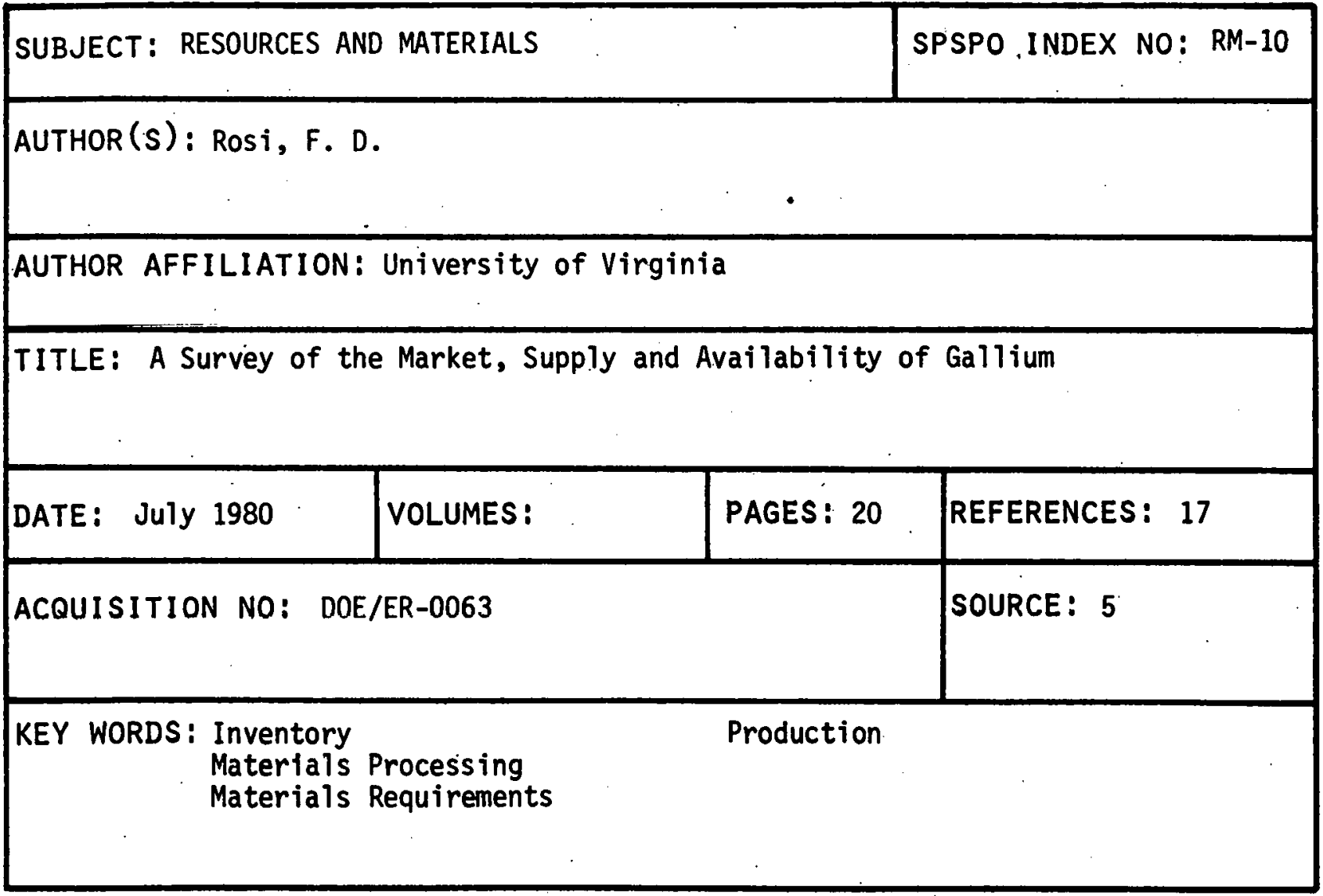




\begin{tabular}{|c|c|c|c|c|c|}
\hline SUBJECT: & \multicolumn{3}{|c|}{ RESOURCES AND MATERIALS } & \multicolumn{2}{|r|}{ SPSPO INDEX NO: RM-7 } \\
\hline AUTHOR (s): & \multicolumn{5}{|c|}{$\begin{array}{l}\text { Teeter, R. R. } \\
\text { Jamieson, W. M. }\end{array}$} \\
\hline \multicolumn{6}{|c|}{ AUTHOR AFFILIATION: Battelle Columbus Laboratories } \\
\hline TITLE : & \multicolumn{5}{|c|}{ Prel iminary Materials Assessment for the Satellite Power System (SPS) } \\
\hline DATE: January & y 1980 & VOLUMES: & PAGES: & 120 & REFERENCES : 26 \\
\hline ACQUISITION & NO: & DOE/ER-0038 & & & SOURCE : \\
\hline KEY WORDS : & $\begin{array}{l}\text { Assessment } \\
\text { Economics } \\
\text { Inventory }\end{array}$ & Methods & & $\begin{array}{l}\text { Mat } \\
\text { Pro } \\
\text { Sel }\end{array}$ & $\begin{array}{l}\text { rials Constraints } \\
\text { luction } \\
\text { ction Methods }\end{array}$ \\
\hline
\end{tabular}

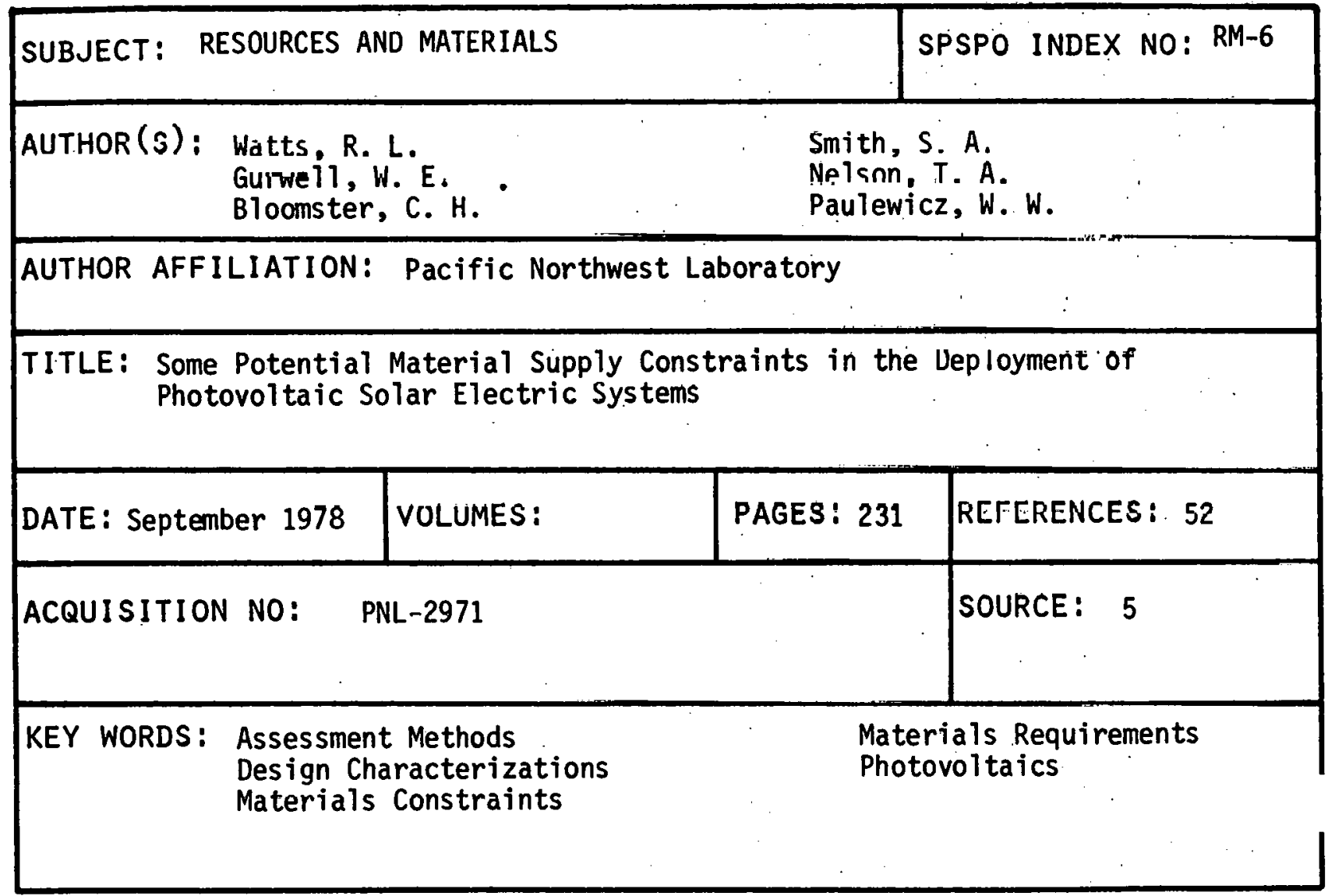




\section{SYSTEMS ANALYSES \\ (CATEGORY SA)}

This category of documents includes reports and papers dealing with SPS and other energy technologies in a broad sense. Most documents address more than one key issue or coricern relating to one or more technologies rather than concentrating on individual issues. As a result, the shorter reports provide summaries rather than in-depth analyses of energy technologies, and the longer reports cover a wide range of topics.

KEY WORDS :

Comparative Assessment

Concept Definition

Concept Review

\section{Costs}

Economics

Energy Balance

Energy Conversion

Environment

Ground Receiver

Key Issues

Laser Power

Microwave Transmission

Orbitál Operations

Power Distribution

Resource Analyses

Satellite Stability

Satellite Structure

Social Impacts

Solid State

Space Assembly

Space Environment

Space Reflector

Space Transportation
PAGE NUMBER:

$149,153,161,162,168$

$151,155,160,161,164,165,167,171$

$149,151,152,153,154,156,157,158$,

$161,162,163,165,168,169,170,171$

$150,163,167$

$149,151,152,154,155,156,157,158$, $159,160,162,164,166,169$

151

$149,150,151,152,155,156,157 ; 158$, $159,160,163,164,165,169,170,171$

$149,150,151,152,153,154,155,156$, $157,159,160,161,162,165,166,168$

$154,164,165,171$

$151,153,154,155,161,162,165,167$, 168

$150,151,157,160$

$150,151,156,157,163,167,169$

$151,158,159,167,170$.

$152,155,158,164,169,170$

$156,161,164$

$152,159,169$

$149,150,151,152,154,155,156,158$,

$159,161,163,165,167,169,170,171$

$149,153,156,157,161,162,165,166$, 168

152

$152,156,157,158,159,160,163,164$, $167,169,170,171$

166

150,160

$149,150,153,154,155,156,158,159$,

$160,162,163,164,165,167,168,170,171$ 
(Systems Analyses

Category SA Continued)

KEY WORDS:

Technology Requirements

Transmission System

REL.ATED CATEGORIES:

A11 Other Categories
PAGE NUMBER:

$149,151,153,154,155,156,158,162,167$, 169,171

$149,150,152,154,155,157,158,159,160$, $163,164,165,166,169,170$ 
AUTHOR(s): Alabama, University of

AUTHOR AFFILIATION: University of Alabama (Huntsville)

TITLE: The Final Proceedings of the Solar Power Satellite

Program Review

\begin{tabular}{|l|l|l|l|}
\hline DATE: JuTY 1980 & VOLUMES: & PAGES: 700 & REFERENCES: None \\
\hline ACQUISITION NO: CONF-800491 & SOURCE: 5 \\
\hline
\end{tabular}

KEY WORDS: Comparative Assessment

Satellite Structures

Concept Review

Social Impacts

Environment

Transmission System

SUBJECT: SYSTEMS ANALYSES

SPSPO INDEX NO: SA-44

AUTHOR(S): Argonne National Laboratory

AUTHOR AFFILIATION: Argonne National Laboratory

TITLE: Survey and Documentation of Emerging Technologies for the Satellite Power System (SPS)

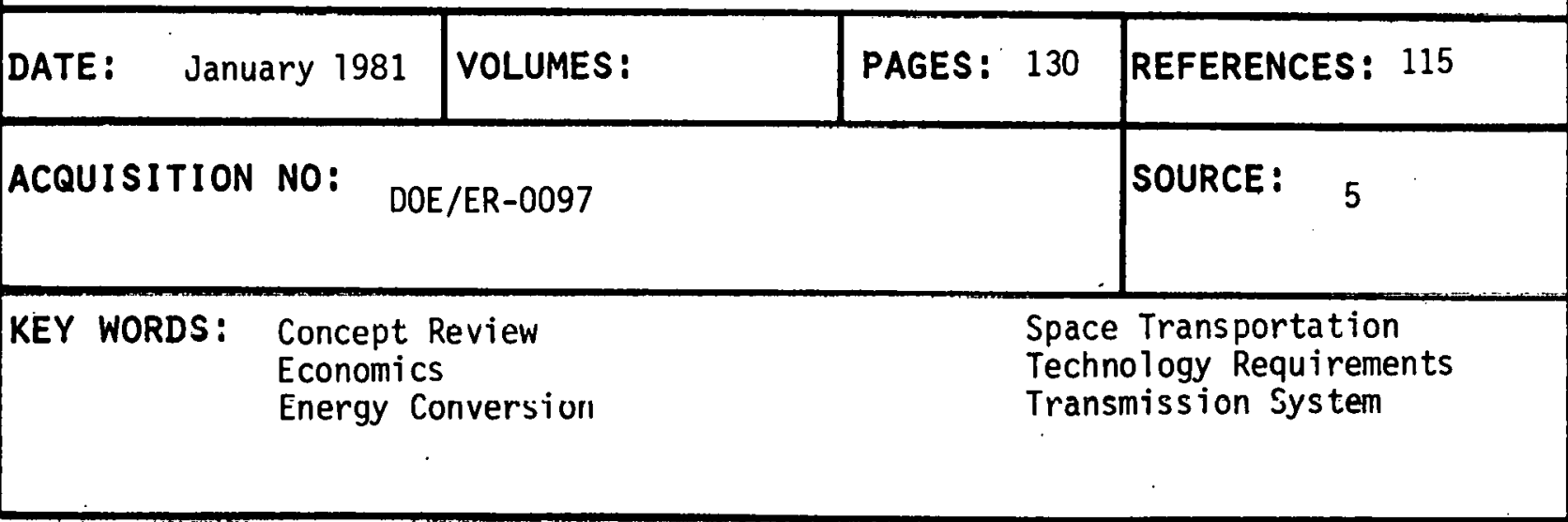


SUBJECT: SYSTEMS ANALYSES

$\operatorname{AUTHOR}(S)$ : Billman, K. W. (Ed.)

AUTHOR AFFILIATION: Ames Research Center

TITLE: Radiation Energy Conversion in Space

\begin{tabular}{|l|l|l|l|}
\hline DATE: 1978 & VOLUMES: & PAGES: 688 & REFERENCES: 791 \\
\hline ACQUISITION NO: & SSBN 0-915928-26-4 \\
& $\begin{array}{l}\text { Progress in Astronauttcs and } \\
\text { Aeronautics, Volume 61 }\end{array}$ & \\
\hline KEY WORDS: & $\begin{array}{l}\text { Energy Conversion } \\
\text { Laser Power } \\
\text { Microwave Transmission }\end{array}$ & $\begin{array}{l}\text { Space Transportation } \\
\text { Transmission System }\end{array}$ \\
\hline
\end{tabular}

SUBJECT: SYSTEMS ANALYSES

SPSSPO INDEXX NO: SA-36

$\operatorname{AUTHOR}(3)$ : Billillari, K. W.

Gilbreath, W. P.

Bowen, S. W.

AUTHOR AFFILIATION: NASA Ames Research Center

TITLE: Space Reflector Technology and Its System Implications

\begin{tabular}{|c|c|c|c|c|}
\hline DATE: Februa & ary 1979 & VOLUMES: & PAGES: 17 & REFERENCES: 18 \\
\hline \multicolumn{4}{|c|}{ ACQUISITION NO: $79-0545$. } & SOURCE : \\
\hline KEY WORDS: & $\begin{array}{l}\text { Costs } \\
\text { Energy co } \\
\text { Environme }\end{array}$ & $\begin{array}{l}\text { onversion } \\
\text { ent }\end{array}$ & & $\begin{array}{l}\text { 11ite Structures } \\
\text { - Reflector } \\
\text { e Transportation }\end{array}$ \\
\hline
\end{tabular}




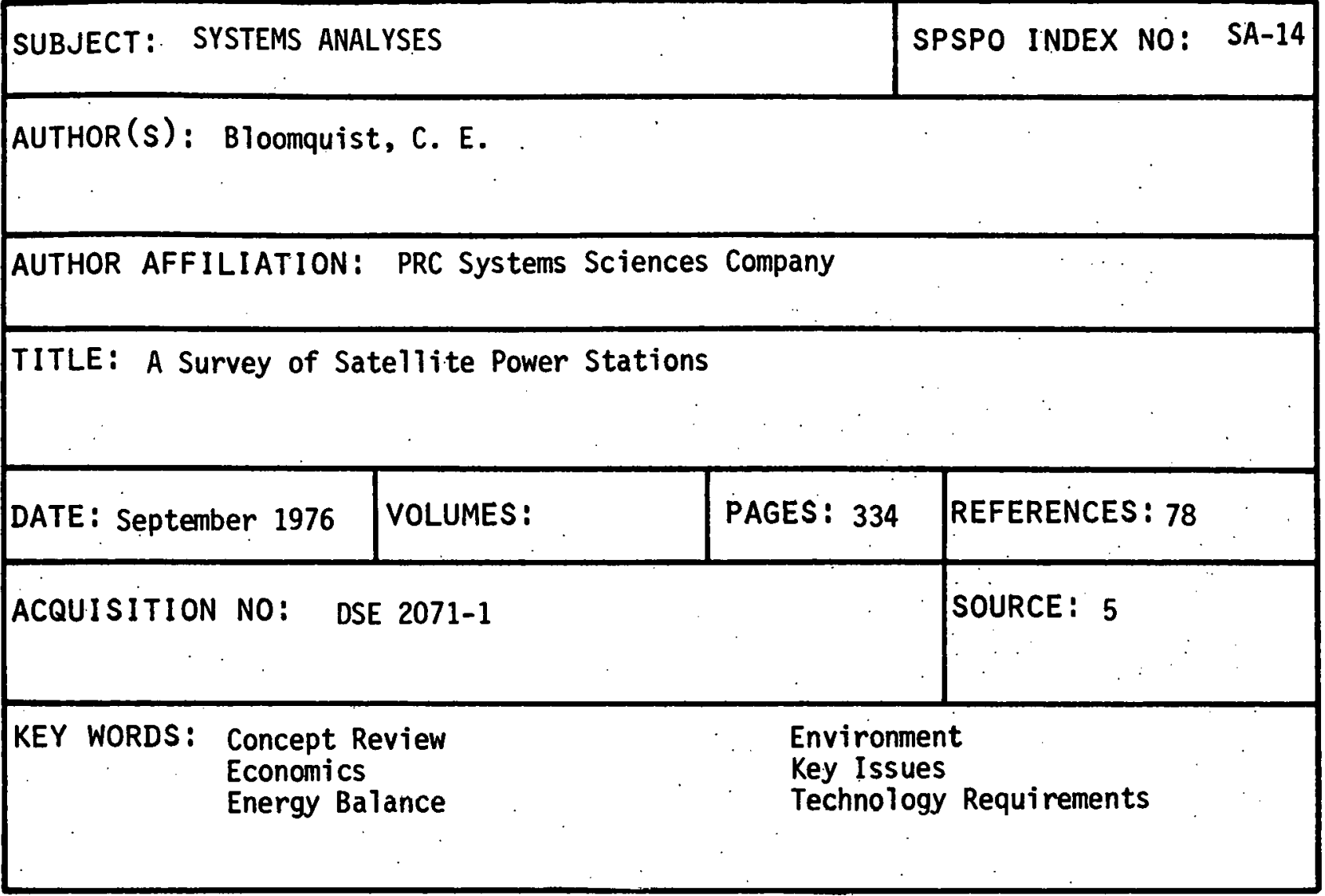

\begin{tabular}{|l|l|}
\hline SUBJECT: SYSTEMS ANALYSES & SPSPO INDEX NO: SA-30 \\
\hline AUTHOR(S): Boeing Aerospace Company & \\
\hline AUTHOR AFF ILIATION: Boeing Aerospace Company & \\
\hline TITLE: Solar Power Satellite System Definition Study, Phase III \\
EXecutive Sumary
\end{tabular}


SUBJECT: SYSTEMS ANALYSES

AUTHOR (s): Brown, W. C.

AUTHOR AFFILIATION: Raytheon Company

TITLE :

Satellite Power Stations: A New Source of Energy?

DATE : March 1973

VOLUMES:

PAGES: 10 REFERENCES: 30

ACQUISITION NO:

Spectrum; Vol. 10, No. 3, pp. 38-47

SOURCE :

6

$\begin{array}{ll}\text { KEY WORDS: } & \text { Concept Review } \\ & \text { Economics } \\ & \text { Energy Conversion }\end{array}$

Environment

Transmission System

Energy Conversion

SPSPO INDEX NO: SA-3

SUBJECT:

SYSTEMS ANALYSES
AUTHOR(s): Cantafto, L.J.
Chobotov, V. A.
Wolfc, M. G.

AUTHOR AFFILIATION:

Aerospace Corporation

TITLE: Photovoltaic, Gravitationally-Stabilized Solid-State Satellite Solar Power Station (GSS ${ }^{4}$ PS)

DATE: March 1977

VOLUMES:

PAGES : 15

REFERENCES :

32

ACQUISITION NO: AIAA-77-511

SOURCE :

KEY WORDS: Power Distribution

Satellite Stability

Solid State

Satellite Structures

Space Assembly 


\begin{tabular}{|c|c|c|c|c|c|}
\hline SUBJECT: $\quad S$ & SYSTEMS & NALYSES & & & SPO INDEX NO: $S$ \\
\hline $\begin{array}{c}\operatorname{AUTHOR}(S): \\
\text { E }\end{array}$ & Energy, 1 & .S. Departme & & & . \\
\hline AUTHOR AFFIL & IATION: & U.S. Depar & iergy & & \\
\hline TITLE: & $\begin{array}{l}\text { Satellit } \\
\text { Program }\end{array}$ & $\begin{array}{l}\text { Power Syst } \\
\text { reliminary }\end{array}$ & oncept De & elop & ent and Evaulation \\
\hline DATE: Septembe & 1979 & VOLUMES : & PAGES: & 14 & REFERENCES : 22 \\
\hline ACQUISITION & NO: $D O E$ & ER-0041 , & & & SOURCE : \\
\hline KEY WORDS : & $\begin{array}{l}\text { Comparat } \\
\text { Concept } \\
\text { Environm }\end{array}$ & $\begin{array}{l}\text { ve Assessme } \\
\text { eview } \\
\text { nt }\end{array}$ & & $\begin{array}{l}\text { Key } \\
\text { Soc } \\
\text { Tect }\end{array}$ & $\begin{array}{l}\text { Issues } \\
\text { al Impacts } \\
\text { nology Requirements }\end{array}$ \\
\hline
\end{tabular}

SUBJECT: SYSTEMS ANALYSES

SPSPO INDEX NO: SA-24

AUTHOR(S): Energy, U. S. Department of

AUTHOR AFFILIATION: U. S. Department of Energy

TITLE: Program Assessment Report Statement of Findings

DATE: November 1980

VOLUMES:

PAGES: 76

REFERENCES: 50

ACQUISITION NO: DOE/ER-0085

SOURCE: 5

KEY WORDS: Comparative Assessment

Concept Review

Environment

Key Issues

Social Impacts.

Space Transportation 
AUTHOR(S): Energy Research and Development Administration

AUTHOR AFFILIATION: Energy Research and Development Administration

TITLE:

Final Report of the ERDA Task Group on Satellite Power Stations

\begin{tabular}{|c|c|c|c|c|c|}
\hline \multicolumn{2}{|c|}{ DATE : November 1976} & VOLUMES : & PAGES: 29 & REFERENCES: & None \\
\hline ACQUISITION & NO: & ERDA-76/148 & & SOURCE: & 13 \\
\hline KEY WORDS: & $\begin{array}{l}\text { Concept } \\
\text { Economi } \\
\text { Environr }\end{array}$ & $\begin{array}{l}\text { leview } \\
\text { nt }\end{array}$ & & Key Issues & \\
\hline
\end{tabular}

SUBJECT: SYSTEMS ANALYSES

SPSPO INDEX NO: SA-33

$\operatorname{AUTHOR}(S)$ : Glaser, P. E.

AUTHOR AFFILIATION: Arthur D. Little, Inc.

TITLE: Power From the Sun: Its Future

DATE: November 1968

VOLUMES:

\begin{tabular}{l|l|l} 
PAGES: 5 & REFERENCES: 15 \\
\hline
\end{tabular}

ACQUISITION NO: By Title, Vol. 162, No.. 3856

SOURCE: 7

KEY WORDS: Concept Review Ground Receiver

Satellite Structures
Space Transportation

Technology Requirements

Transmission System 
$\begin{array}{lll}\text { AUTHOR (S): } & \text { Glaser, P. E. } \cdot(1) \\ & \text { Maynard, 0. E. (2) } \\ & \text { Mackovciak, J. (3) }\end{array}$

Ralph, E. L. (4)

AUTHOR AFFILIATION:

(1) A. D. Little, Inc.

(2) Raytheon Co.

(3) Grumman Aerospace Corp.

(4) Textron Inc.

TITLE: Feasibility Study of Satellite Solar Power Station

1

DATE: February 1974

VOLUMES :

\begin{tabular}{l|l} 
PAGES : 199 & REFERENCES : 72
\end{tabular}

ACQUISITION NO: NASA CR-2357

SOURCE: 4

KEY WORDS: Concept Definition

Economics

Environment

Key Issues

Satellite Structures

Technology Requirements

SUBJECT: SYSTEMS ANALYSES

AUTHOR(S): Glaser, P. E.

AUTHOR AFFILIATION: A. D. Little, Inc.

TITLE: Perspectives on Satellite Solar Power

DATE: January 1977

VOLUMES:

PAGES: 12

REFERENCES: 31

ACQUISITION NO: By Title; AIAA 13th Annual Meeting (Washington, D. C.)

SOURCE :

KEY WORDS: Concept Definition

Energy Conversion

Power Distribution
Space Transportation

Technology Requirements

Transmission system 
AUTHOR(S): Glaser, P. E.

AUTHOR AFFILIATION: Arthur D. Little, Inc.

TITLE: Solar Power From Satellites

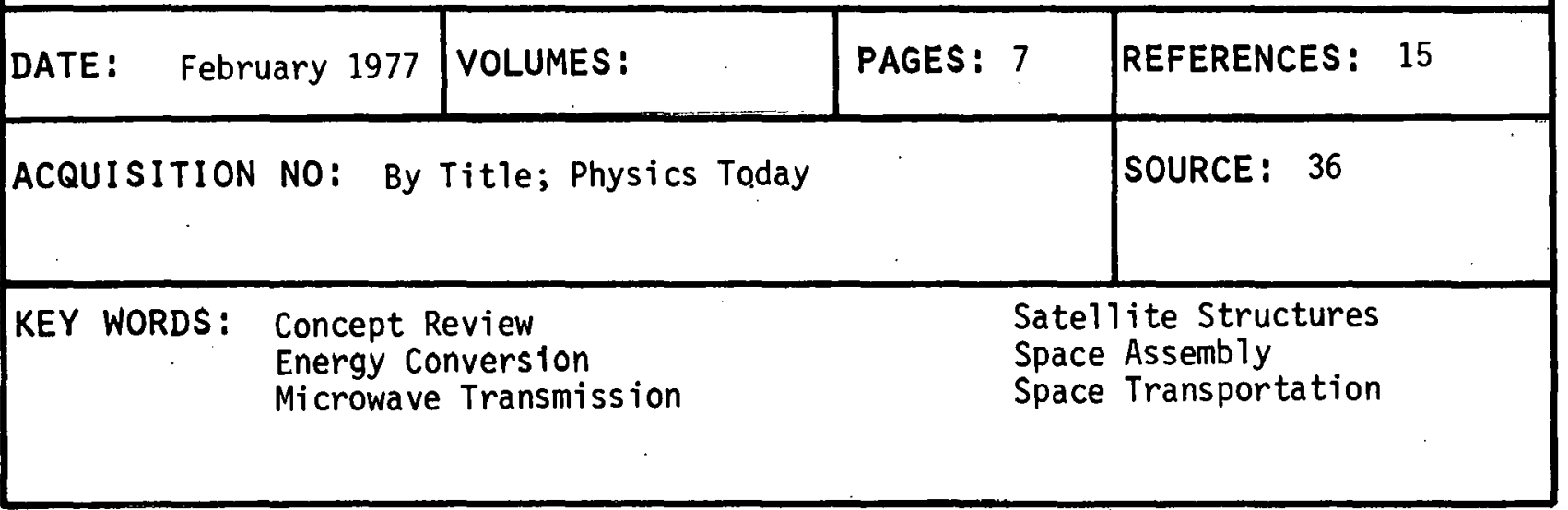

SUBJECT: SYSTEMS ANALYSES

SPSPO INDEX NO: SA-4I

AUTHOR(s): Glaser, P. E.

AUTHOR AFFILIATION: Arthur D. Little, Inc.

TITLE: The Development of Solar Power Satellites

\begin{tabular}{|l|l|l|l|}
\hline DATE: 1979 & VOLUMES: & PAGES: 48 & REFERENCES: 49 \\
\hline ACQUISITION NO: ISBN-0-12-014902-8 & SOURCE: 40 \\
\hline KEY WORDS: $\begin{array}{l}\text { Concept Review } \\
\text { Economics } \\
\text { Environment }\end{array}$ & $\begin{array}{l}\text { Resource Analysis } \\
\text { Social Impacts } \\
\text { Technology Requirements }\end{array}$ \\
\hline
\end{tabular}




\section{SUBJECT:}

AUTHOR(s): Glaser, P. E. (1)

Hanley, G. M. (2)

Nansen, R. H. (3)

Kline, R. L. (4)

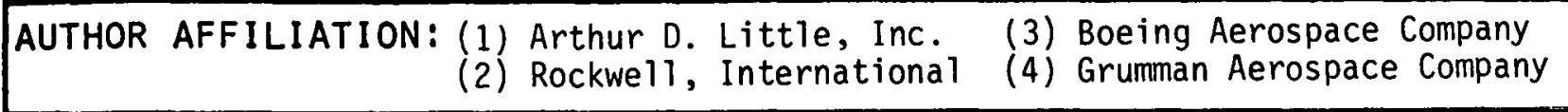

TITLE: $\quad$ First Steps to the Solar Power Satellite

\begin{tabular}{|l|l|l|l|}
\hline DATE: May 1979 & VOLUMES: & PAGES: 7 & REFERENCES : None \\
\hline ACQUISITION NO: 0018-9235/0500-0052 & SOURCE: \\
\hline KEY WORDS: $\begin{array}{l}\text { Concept Review } \\
\begin{array}{l}\text { Energy Conversion Space Assembly } \\
\text { Laser Power }\end{array}\end{array}$ & \begin{tabular}{l} 
Transmission System \\
\hline
\end{tabular} \\
\hline
\end{tabular}

SUBJECT: SYSTEMS ANALYSES

SPSPO INDEX NO: SA-38

$\operatorname{AUTHOR}(S): \quad$ Glaser, $\ddot{\mathrm{P}} . \mathrm{E}$.

AUTHOR AFFILIATION: Arthur D. Little, Inc.

TITLE: The Earth Benefits of Solar Power Satellites

\begin{tabular}{|c|c|c|c|c|}
\hline DATE: 1980 & & VOLUMES : & PAGES: $\cdot 30$ & REFERENCES : 36 \\
\hline ACQUISITION & NO: & $\begin{array}{l}\text { 0191-9067/80/010009-30 } \\
\text { Space Solar Power Review, } \\
\text { pp. } 9-38\end{array}$ & Vol. 1 & SOURCE : 33 \\
\hline KEY WORDS: & \multicolumn{2}{|c|}{$\begin{array}{l}\text { Concept Review } \\
\text { Economics } \\
\text { Environment }\end{array}$} & \multicolumn{2}{|c|}{$\begin{array}{l}\text { Laser Power } \\
\text { Microwave Transmission } \\
\text { Social Impacts }\end{array}$} \\
\hline
\end{tabular}


SUBJECT: SYSTEMS ANALYSES

AUTHOR(s): Hanley, G. M.

\section{AUTHOR AFFILIATION: Rockwell International}

TITLE: Rockwell Satellite Power System (SPS) Concept Definition Studies

\begin{tabular}{|l|l|l|l|}
\hline DATE: 1980 & VOLUMES: & PAGES: 17 & REFERENCES: 2 \\
\hline ACQUISITION NO: $\begin{array}{l}0191-9067 / 80 / 010079-17 \\
\text { Space Solar Power ReV1ew, Vol. 1, } \\
\text { pp. 79-95 }\end{array}$ & SOURCE: 33 \\
\hline KEY WORDS: $\begin{array}{l}\text { Concept Review } \\
\text { Energy Conversion } \\
\text { Power Distribution }\end{array}$ & $\begin{array}{l}\text { Satellite Structures } \\
\text { Space Assembly } \\
\text { Space Transportation }\end{array}$ \\
\hline
\end{tabular}

\begin{tabular}{|c|c|}
\hline SUBJECT : & SYSTEMS ANALYSES \\
\hline AUTHOR (S) & $\begin{array}{l}\text { Hazelrigg, G. A } \\
\text { Adornato, R. J. } \\
\text { Wendel 1, R.. A. } \\
\text { Glaser, P. F.. }\end{array}$ \\
\hline
\end{tabular}

AUTHOR AFFILIATION: (1) ECON, Inc. (3) Raytheon Company

(2) Grumman Aerospace Corp. (4) A. D. Little, Inc.

TITLE: $\quad$ Space-Based Solar Power Conversion and Delivery Systenlls Study

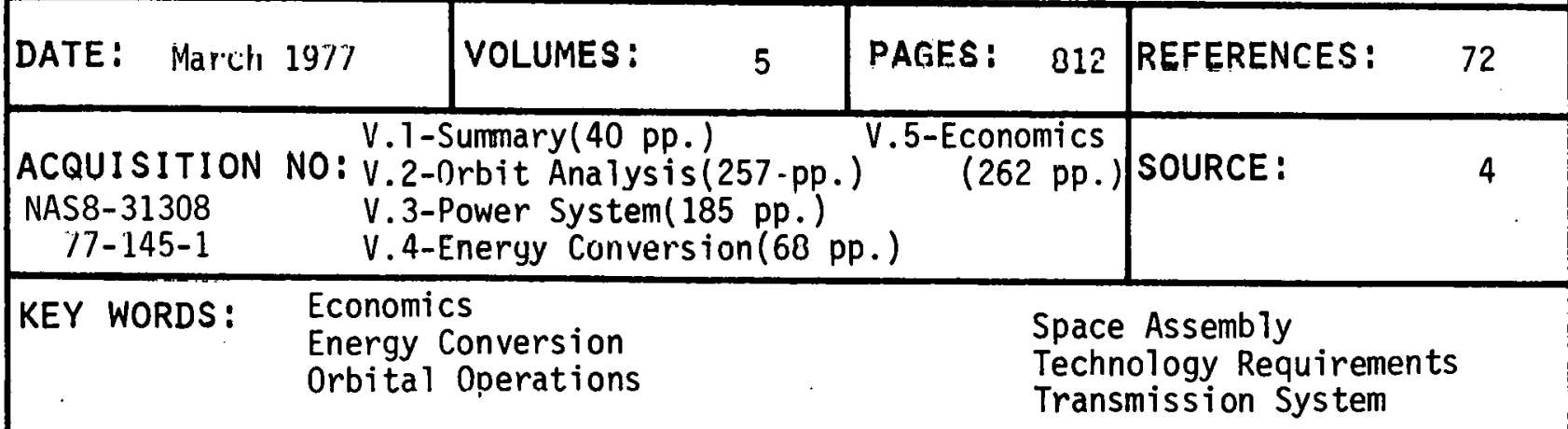


$\operatorname{AUTHOR}(S)$ : Johnson Space Center.

AUTHOR AFFILIATION: National Aeronautics and Space Administration

TITLE: Initial Technical, Environmental and Economic Evaluation of Space Solar Power Concepts

\begin{tabular}{|l|l|l|l|}
\hline DATE: August 1976 & VolUMES: 2 & PAGES: 986 & REFERENCES: 72 \\
\hline ACQUISITION NO: JSC-11568 & SOURCE: \\
V. 1 - Summary (119 pp.) & & 4 \\
\hline
\end{tabular}

KEY WORDS: Economics

Energy Conversion

Orbital Operations

Environment

Space Transportation

Transmission Sys tem

SUBJECT:

SYSTEMS ANALYSES

SPSPO INDEX NO: SA-4

AUTHOR(S): Johnson Space Center

AUTHOR AFFILIATION: National Aeronautics and Space Administration

TITLE: Preliminary Assessment of Technology Advancement Requirement for Space Solar Power

DATE: March 1977

VOLUMES:

PAGES: 200

REFERENCES: None

ACQUISITION NO: JSC- 12702

SOURCE:

1

KEY WORDS: Energy Conversion

Satellite Stability

Satellite Structures

Space Assembly

Transmission System 


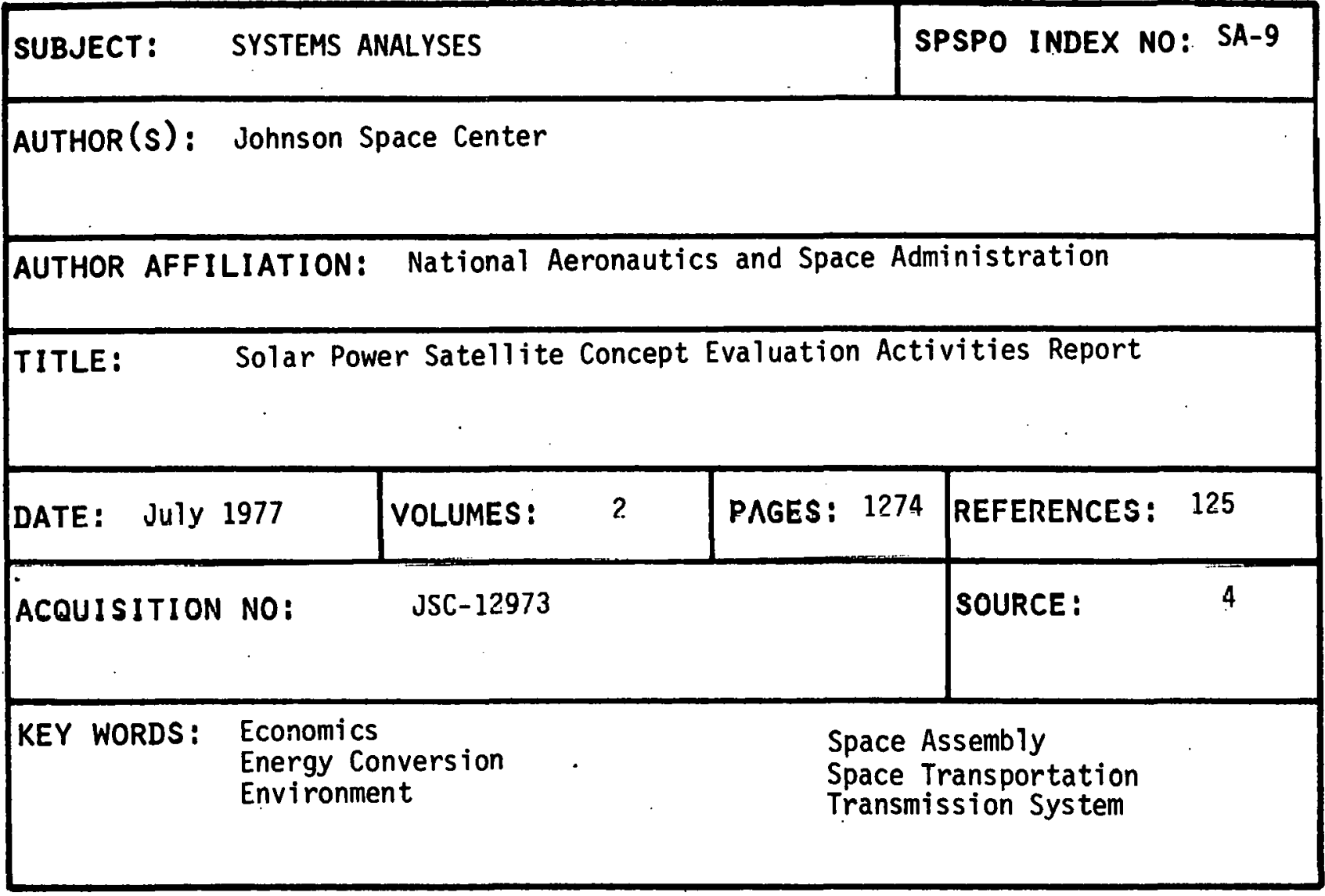

\section{SUBJECT: - SYSTEMS ANALYSES}

SPSPO INDEX NO: SA-1

$\operatorname{AUTHOR}(s)$ : Jones, W. $s$.

Hunter, M. W.

AUTHOR AFFILIATION: Lockheed Missiles and Space Company.

TITLE: Solar Power Satellites: The Laser Option

DATE : March 1979 VOLUMES: \begin{tabular}{l|l|l} 
PAGES: 2 & REFERENCES: None \\
\hline
\end{tabular} ACQUISITION NO: Astronautics and Aeronautics

Astronautics and
Vol 17, No. 3 SOURCE : 2 pp. 59,67

KEY WORDS: Concept Definition

Laser Power

Space Reflector 
SUBJECT: SYSTEMS ANALYSES

AUTHOR (s): Koomanoff, F. A.

AUTHOR AFFILIATION: U. S. Department of Energy

TITLE: Status of the Satellite Power System (SPS) Concept Development and Evaluation Program

\begin{tabular}{|c|l|l|l|}
\hline DATE: December 1978 & VOLUMES: & PAGES: 17. & REFERENCES: None \\
\hline $\begin{array}{ll}\text { ACQUISITION NO: By Title; Anglo-American Workshop } \\
\text { On Energy and Aerospace (London) }\end{array}$ & SOURCE: 2 \\
\hline KEY WORDS: & $\begin{array}{l}\text { Concept Review } \\
\text { Environment } \\
\text { Key Issues }\end{array}$ & $\begin{array}{l}\text { Resource Analysis } \\
\text { Satellite Structures } \\
\text { Social Impacts }\end{array}$ \\
\hline
\end{tabular}

\section{SUBJECT: SYSTEMS ANALYSES}

AUTHOR(S): Koomanoff, F.A. (1)

Sandah1, C. A.

AUTHOR AFFILIATION: (1) U. S. Department of Energy

(2) Argonne Natinnal l.ahoratory

TITLE: Status of Satellite Power System Concept Development and Evaluation Program

\begin{tabular}{|l|l|l|l|}
\hline DATE: September 1979 & VOLUMES: & PAGES: 17 & REFERENCES: 22 \\
\hline ACQUISITION NO: $\begin{array}{l}\text { By Title (30th Congress of the } \\
\text { International Astronautical Federation } \\
\text { Munich, FRG) }\end{array}$ & SOURCE: 42 \\
\hline KEY WORDS: $\begin{array}{l}\text { Comparative Assessment } \\
\begin{array}{l}\text { Concept Definition } \\
\text { Environment }\end{array}\end{array}$ & $\begin{array}{l}\text { Key Issues } \\
\text { Satellite Structures } \\
\text { Social Impacts }\end{array}$ \\
\hline
\end{tabular}


AUTHOR(s): Koomanoff, F.A. (1)

Sandah1, C. A.

AUTHOR AFFILIATION: (1) U. S. Department of Energy

(2) Argonne National Laboratory

TITLE: Status of the Satellite Power System Concept Development and Evaluation Program

DATE: 1980 VOLUMES: PAGES: 11 REFERENCES: 22

ACQUISITION NO: $0191-9067 / 80 / 010067$, Space Solar SOURCE :

Power Review

KEY WORDS: Comparative Assessment

Concept Review

Environment

Key Issues

Social Impacts

Technology Requirements

SUBJECT: SYSTEMS ANALYSES

SPSPO INDEX NO: SA-23

AUTHOR (s): Koomanoff, F. A.

AUTHOR AFFILIATION: U. S. Department of Energy

TITLE: Satellite Power System Concept Development and Evaluation Program Findings

DATE: September 1980

VOLUMES :

PAGES: 33

REFERENCES: 11

ACQUISITION NO: By Title; 31st Congress of the International Astronautical Federation (Tokyo)

KEY WORDS: Concept Review

Economics

Environment

Key Issues

Social Impacts

Space Transportation 
SUBJECT: SYSTEMS ANALYSES

SPSPO INDEX NO: SA-16

$\operatorname{AUTHOR}(s)$ : Kraft, C. C.

AUTHOR AFFILIATION: National Aeronautics and Space Administration

TITLE: The Solar Power Satellite Concept - The Past Decade and the Next Decade

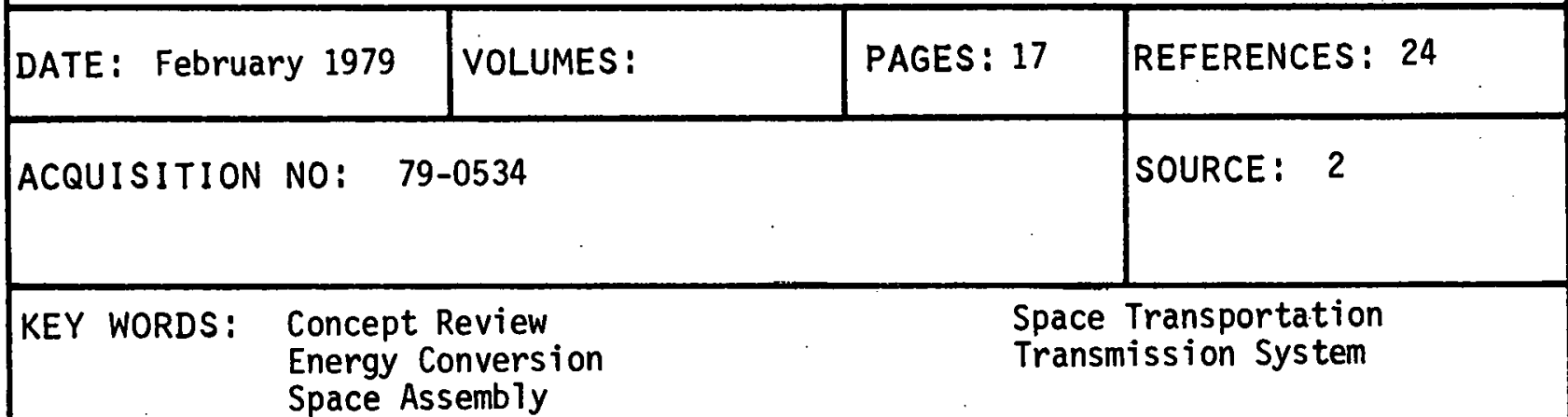

SUBJECT: SYSTEMS ANALYSES

SPSPO INDEX NO: SA-39

$\operatorname{AUTHOR}(s)$ : Kraft, C. C.

Piland, R. 0.

AUTHOR AFFILIATION: National Aeronautics and Space Administration

TITLE: The Solar Power Satellite Concept - The Past Decade and the Next Decade

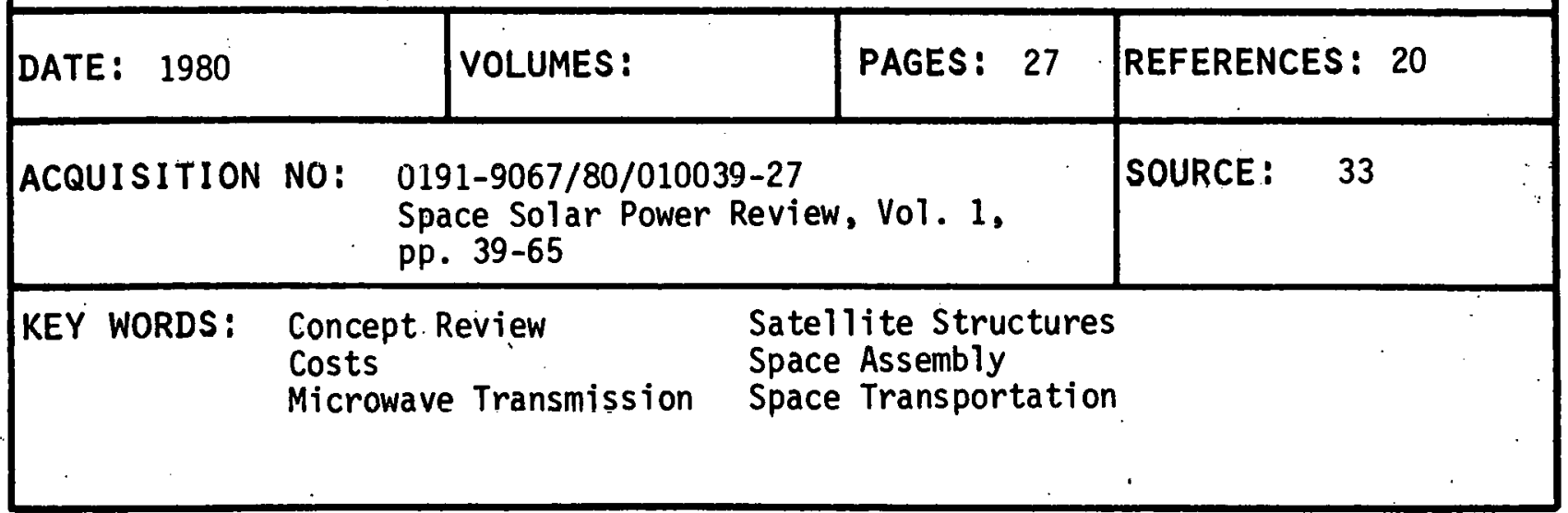




\section{SUBJECT: SYSTEMS ANALYSES}

$\operatorname{AUTHOR}(S)$ : Marshall Space Flight Center

AUTHOR AFFILIATION: National Aeronautics and Space Administration

TITLE: Satellite Power System Engineering and Economic Analys is

\begin{tabular}{|l|l|l|l|}
\hline DATE: November $19 / 6$ & VOLUMES: & PAGES: 280 & REFERENCES: 26 \\
\hline ACQUISITION NO: & NASA-TM-X-73344 & SOURCE: \\
\hline KEY WORDS: $\begin{array}{l}\text { Concept Definition } \\
\text { Economics } \\
\text { Ground Receiver }\end{array}$ & $\begin{array}{l}\text { Power Distribution } \\
\text { Resource Analysis } \\
\text { Transmission System }\end{array}$ \\
& & \\
\hline
\end{tabular}

SUBJECT: SYSTEMS ANAI YSES

SPSPO INDEX NO: SA-12

$\operatorname{AUTHOR}(s)$ : McRae, W. V.

AUTHOR AFFILIATION: Rockwell International

TITLE: Satellite Power System (SPS) Feasibility Study Final Report

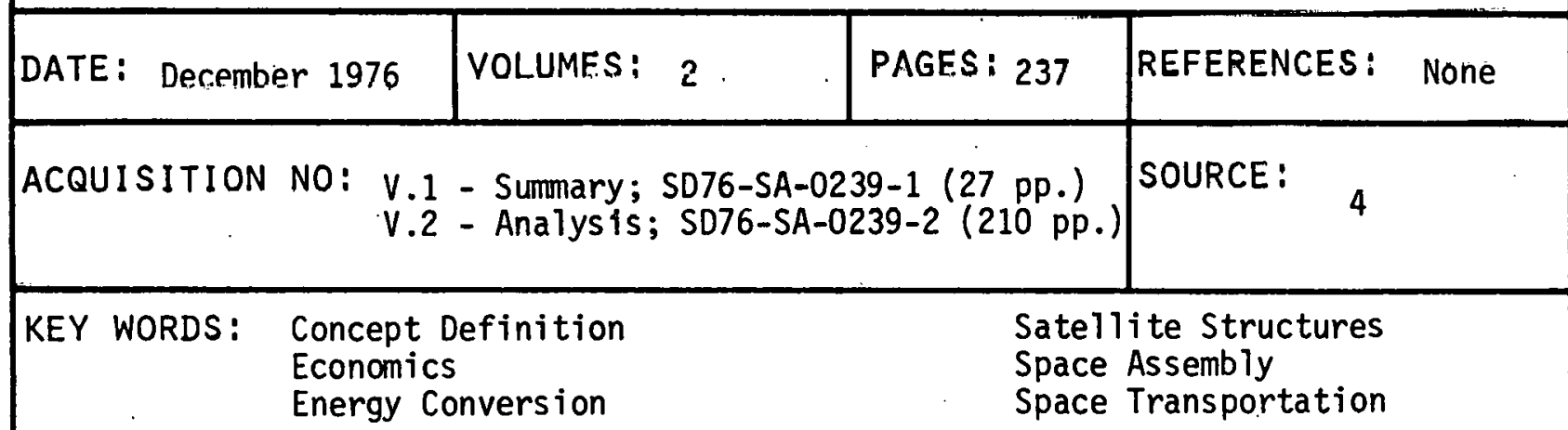


AUTHOR (s): Moses, H.

AUTHOR AFFILIATION: U. S. Department of Energy

TITLE: Impacts of Satellite Power System Technology

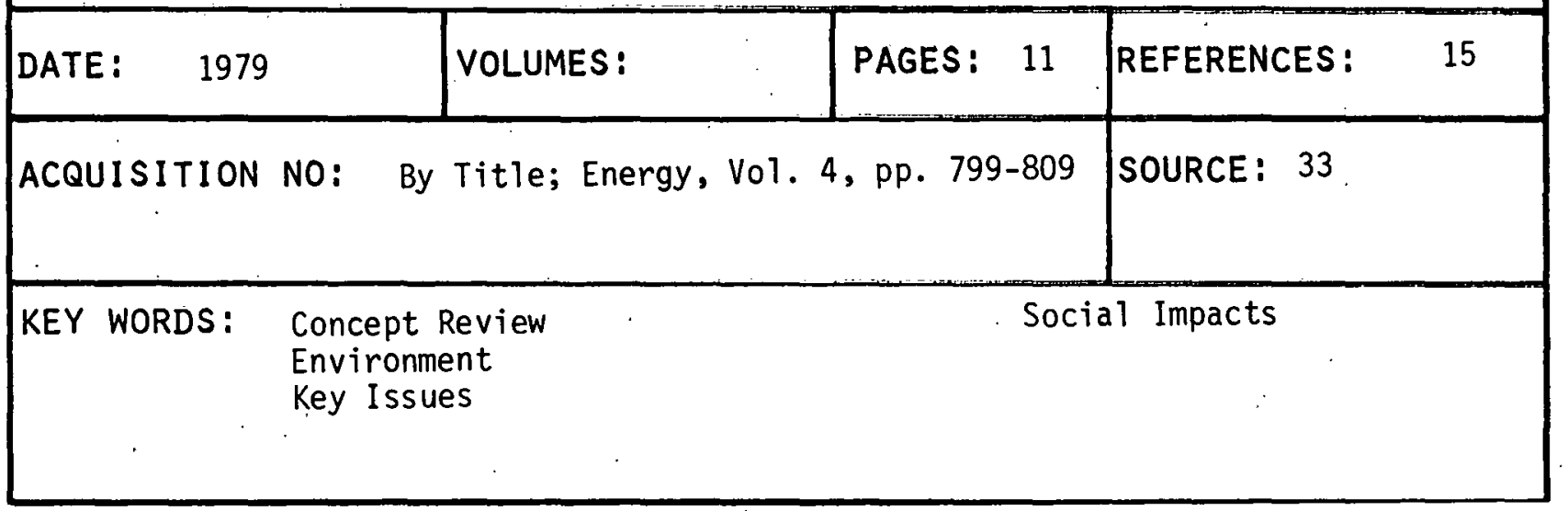

\begin{tabular}{|l|l|}
\hline SUBJECT: SYSTEMS ANALYSES & SPSPO INDEX NO: SA-43 \\
\hline
\end{tabular}

AUTHOR(S): National Aeronautics and Space Administration

AUTHOR AFFILIATION: National Aeronautics and Space Administration

TITLE: Satellite Power System Concept Development and Evaluation Program Technical Assessment Report

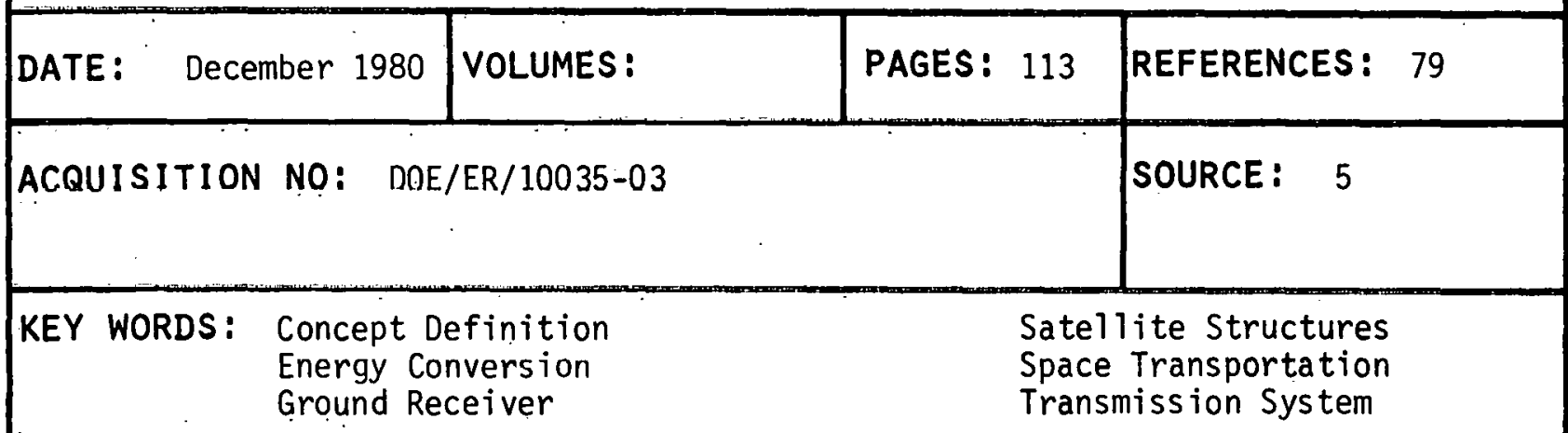


SUBJECT: SYSTEMS ANALYSES

$\operatorname{AUTHOR}(s)$ :

National Research Council

AUTHOR AFFILIATION: National Academy of Sciences

TITLE: NOT AVAILABLE - Committee Report on SPS expected to be published in Mid-1981

DATE:

VOLUMES:

PAGES :

REFERENCES :

ACQUISITION NO:

SOURCE :

27

KEY WORDS :

SUBJECT: SYSTEMS ANALYSES

SPSPO INDEX NO: SA-13

AUTHOR(s): Rice University

AUTHOR AFFILIATION: Rice University

TITLE: Space Solar Power Research Program

\begin{tabular}{|l|l|}
\hline DATE: December 1977 & VOLUMES : \\
\hline
\end{tabular}

ACQUISITION NO: By Title

PAGES: 63 REFERENCES: None

SOURCE : 19

KEY WORDS: Economics

Environment

Social Impacts

Space Environment

Transmission System 


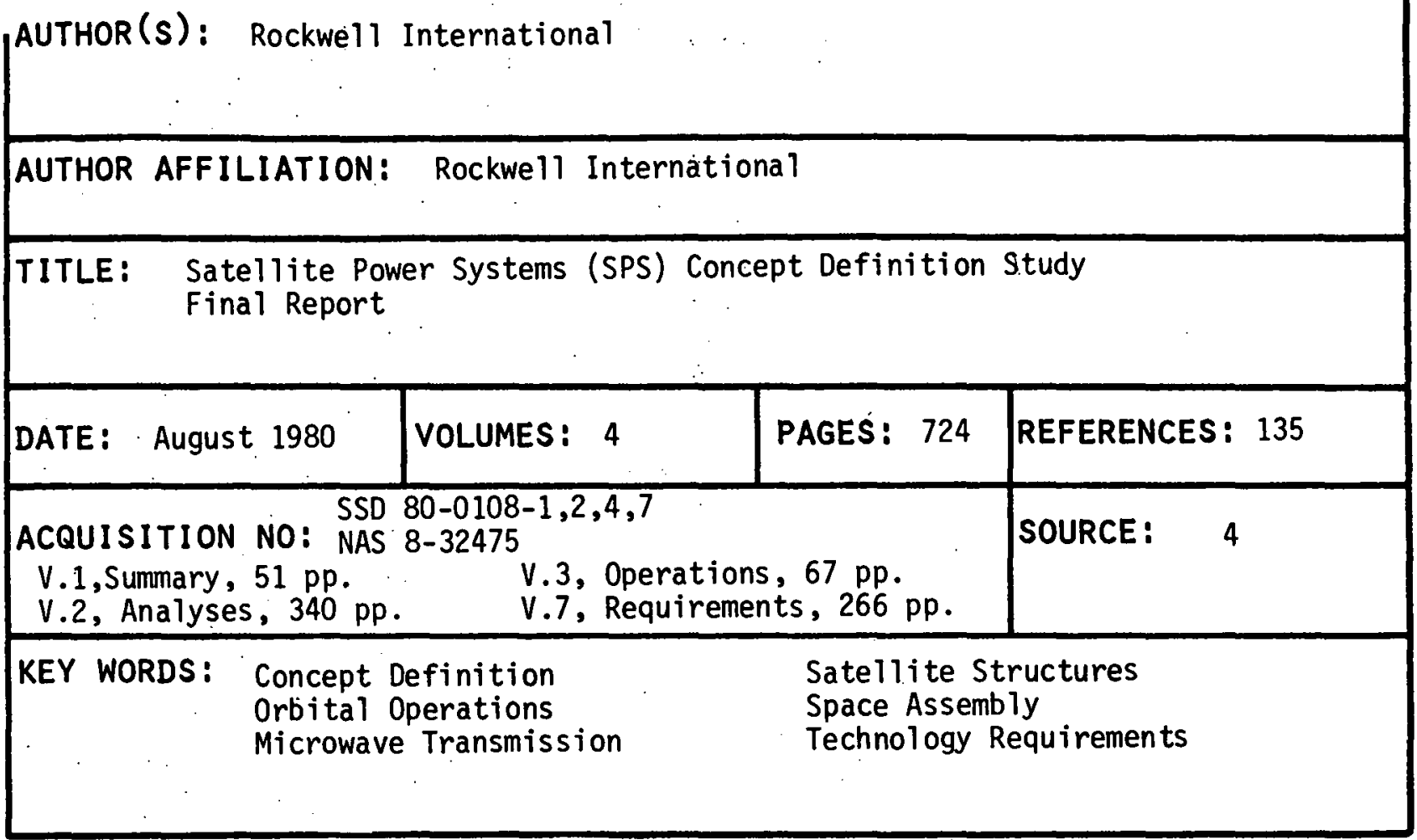

SUBJECT: 'SYSTEMS ANALYSES

AUTHOR(s): Rockwell International

AUTHOR AFFILIATION: Rockwell International

TITLE: Satellite Power Systems (SPS) Concept Definition Study Final Report

DATE: October 1980

VOLUMES: 4

PAGES: 792 REFERENCES: 2

ACQUISITION NO: SSD80-0108-3, 5, 6-1, 6-2

NAS $8-32475$

V.3, Transportation, $87 \mathrm{pp}$.

V.5. Integration, $220 \mathrm{pp}$.

V. $6-1$; Costs, $88 \mathrm{pp}$

V.6-2, Appendix, $397 \mathrm{pp}$.

SOURCE :

KEY WORDS: Concept Definition

Costs

Space Transportation

Key Issues

Technology Requirements 
AUTHOR(S): Schwenk, F. C.

AUTHOR AFFILIATION: National Aeronautics and Space Administration

TITLE: The SPS Concept-An Overview of Status and Outlook

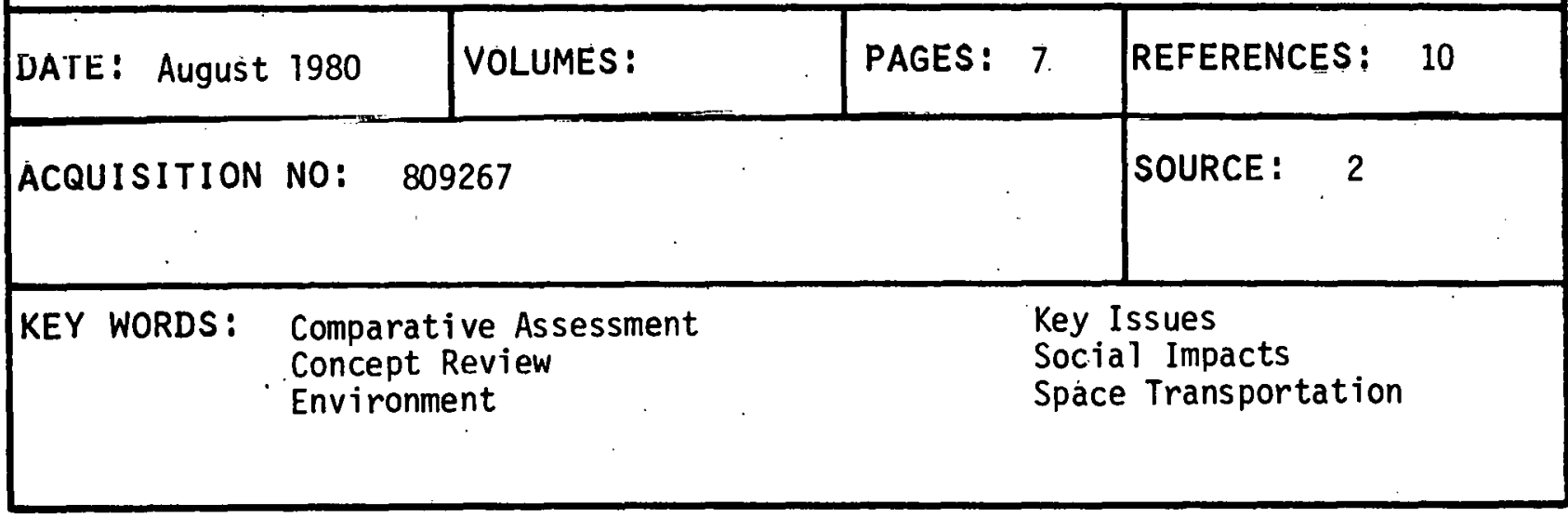

SUBJECT: SYSTEMS ANALYSES

AUTHOR(s): Tcchnology Assessment, Office of

AUTHOR AFFILIATION: U. S. CongresS

TITLE: NOT AVAILABLE - Reporit on SPS expected to be published in spring, 1981

\begin{tabular}{|l|l|l|l|}
\hline DATE: & VULUMES: & PAGES: & REFERENCES: \\
\hline ACQUISITION NO: & SOURCE: 20 \\
\hline KEY WORDS: & & \\
\hline
\end{tabular}




$\begin{aligned} \text { AUTHOR (S): } & \text { Vanke, V. A. } \\ & \text { Lopukhin, V. M. } \\ & \text { Savvin, V. L. }\end{aligned}$

AUTHOR AFFILIATION: M. W. Lomonosov State University (Moscow)

TITLE: Satellite Solar Power Stations

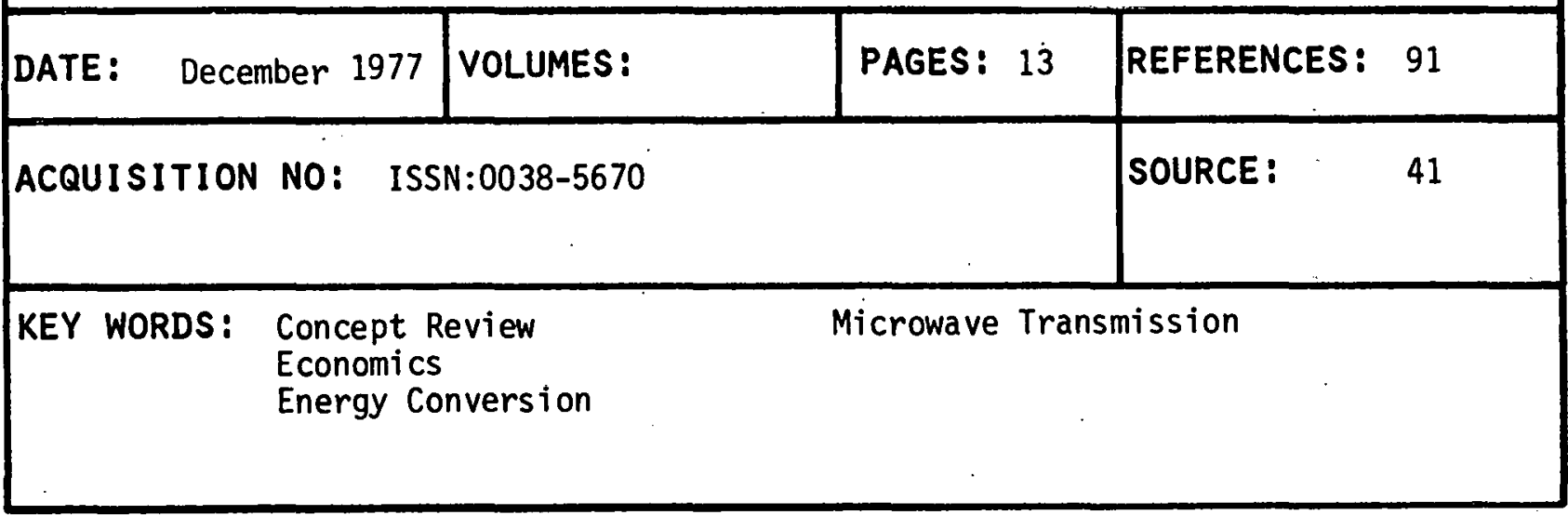

SUBJECT :

SYSTEMS ANALYSES

AUTHOR(S): Wolfe, M. G.

AUTHOR AFFILIATION:

Aerospace Corporation

TITLE :

Application of Stationkept Array Concepts to Satellite Solar Power Station Design

\begin{tabular}{|l|l|}
\hline DATE: November 1976 & VOLUMES: 2 \\
\hline ACQUISITION NO: NA8- 31842 \\
ATR-76(7575)-1 \\
Vol. 1 - Summary (32 pp.) \\
Vol. 2 - Analysis (167 pp.)
\end{tabular}

KEY WORDS: Power Distribution

Satellite Stability

Satellite Structures

Space Assembly

Technology Requirements

Transmission System 
SUBJECT: SYSTEMS ANALYSES

SPSPO INDEX. NO: SA-7. .

AUTHOR(S): Woodcock, G. R.

AUTHOR AFFILIATION: Boeing Aerospace Company

TITLE: Satellite Power Systems for Large-Scale Power Generation

\begin{tabular}{|l|l|l|l|}
\hline DATE: October 1976 & VOLUMES: & PAGES: 11 & REFERENCES: 8 \\
\hline ACQUISITION NO: $\begin{array}{l}\text { By Title; 27th Congress of the } \\
\text { International Astronautical } \\
\text { Federation (Anaheim) }\end{array}$ & $\begin{array}{l}\text { SOURCE: } \\
42\end{array}$ \\
\hline
\end{tabular}

KEY WORDS: Concept Review

Energy Conversion

Satellite Structures

Power Distribution

Space Transportation

Transmission System

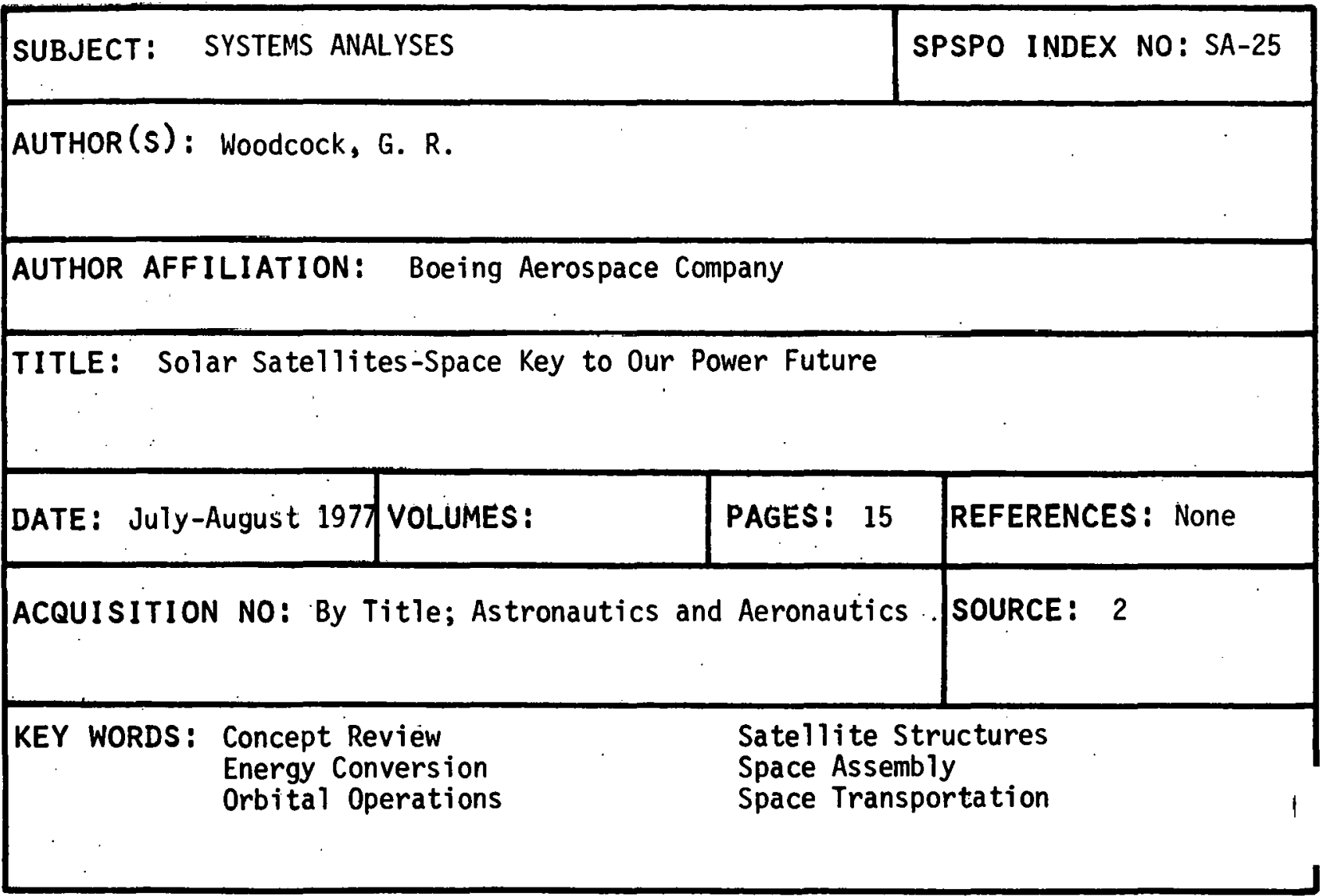




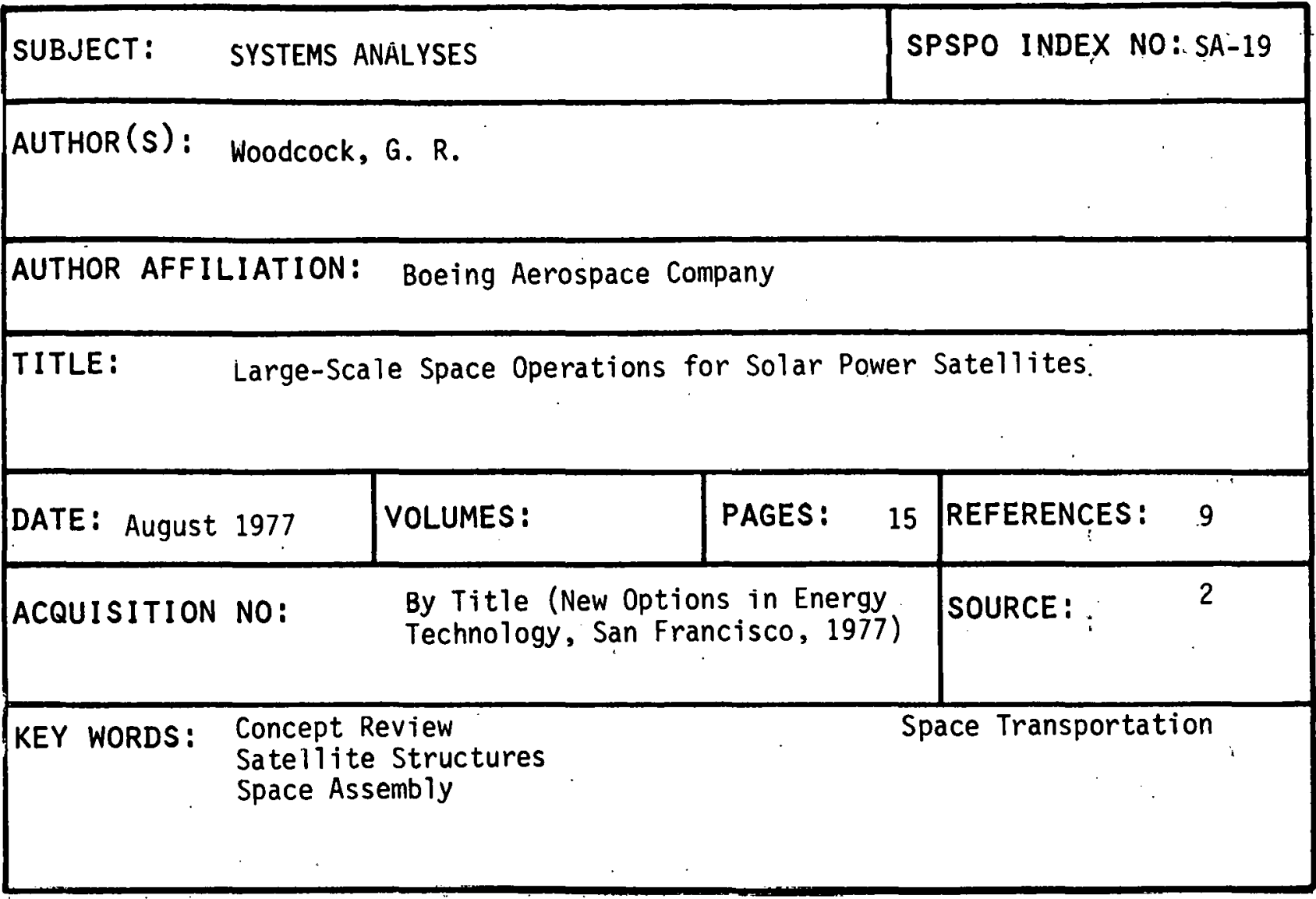

\begin{tabular}{|l|l|}
\hline SUBJECT: SYSTEMS ANALYSES & SPSPO INDEX NO: SA-26 \\
\hline
\end{tabular}

AUTHOR (s): Woodcock, G.

AUTHOR AFFILIATION: Boeing Aerospace Company

TITLE: Solar Power Satellite Systems Definition

\begin{tabular}{|c|c|c|c|c|c|c|}
\hline DATE: & mber 1978 & VOLUMES : & PAGES: 47 & \multicolumn{2}{|c|}{ REFERENCES: } & None \\
\hline ACQUISITION & NO: & \multicolumn{2}{|c|}{$\begin{array}{l}\text { By T1tle (AIAA-RAS Conference on } \\
\text { Energy and Aerospace, London) }\end{array}$} & SOURCE: & & 2 \\
\hline KEY WORDS: & \multicolumn{2}{|c|}{$\begin{array}{l}\text { Concept. Definition } \\
\text { Energy Conversion } \\
\text { Ground Receiver }\end{array}$} & \multicolumn{3}{|c|}{$\begin{array}{l}\text { Satellite Structures } \\
\text { Space Assembly } \\
\text { Technology Requirements }\end{array}$} & \\
\hline
\end{tabular}




\section{THIS PAGE \\ WAS INTENTIONALLY \\ LEFT BLANK}




\section{SYSTEMS ENGINEERING \\ (CATEGORY SE)}

Documents in this category deal with the engineering aspects of the satellite structure which would be assembled in space and the receiving antenna complex which would be built on earth as a part of SPS. Other issues, such as the space-to-earth transmission path, are discussed only to the extent that they may be important factors in engineering principles and design. Note also that reports and papers concentrating on power generation and transmission are listed in a separate category.

KEY WORDS :

Antenna

Antenna Errors

Array Control

Array Design

Cel7 Design

Charged Particles

Command Signals

Concentrators

Converter Station

Debonding

Dipole Assembly

Economics

Efficiency

Electric Charge

Electrostatic Protection

Energy Collection

Energy Conversion

Failure Analysis

Fatigue

Federal Pruyrallis

Fiber Optics

Frequency offset

Geophysics

Ground Station

Industrial Capacity

Interference Effects

Laser Annealing

Load Estimates

Machine Intelligence

Manufacturing Process

Mitigatiun

Models
PAGE NUMBER:

$179,182,183,184,185,191,193$

$180,188,193$

$180,188,190,193$

176,183

176,183

192

$180,188,190$

176,188

175

182

175

183

176

192

191

$175,178,179,183,185,188,191$

$175,178,185,186,188$

176,182

182

183

190

180,190

192

$178,179,180,181,183,185,186,187$, 191

183

190

177

181

189

183

192

192 
(Systems Engineering -

Category SE continued)

KEY WORDS :

Offshore Rectenna

Orbit Maintenance

Orbit Position

Performance

Photovoltaics

Pi lot Beam

Pointing Control

Power B locks

Power Conversion

Power Distribution

Rectenna

Rectenna Alternatives

Rectenna Constructability

Rectenna Costs

Rectenna Design

Rectenna Heat

Rectenna Maintainability

Rectenna Materials

Reliability

Satellite Control

Satellite Maintainability

Satellite Materials

Satellite Structure

Shielding

Solar Collector

Space Automation

Space Construction

Space Transportation

Stability

System Capacity

System Uncertainties

Systems Definition

Transmission Path

Transmiss ion System.

Vibration Test
PAGE NUMBER:

$179,181,191$.

176

189

176

$175, .188$

$180,185, .188,190$

$180,188,190,193$

175,181

175

$175,178,182,185,186,187$

175,181

$179,181,185$

$179,181,192$

$179,185,191,192$

$179,181,185,191,192$

192

$179,181,192$

179,192

181

$176,177,182,184,187,189,190,193$

184,186

187,$189 ; 191$

$177,178,182,183,184,186,187,188$, $189,191,193$

192

175

184,189

$177,178,184,186,187,189$

$176,177,178,182,186,187,189$

181

181

177

$177,182,187$

$180,183,190$

$177,178,182,183,184,185,186,187$.

182

RELATED CATEGORIES:

All Other Categories 


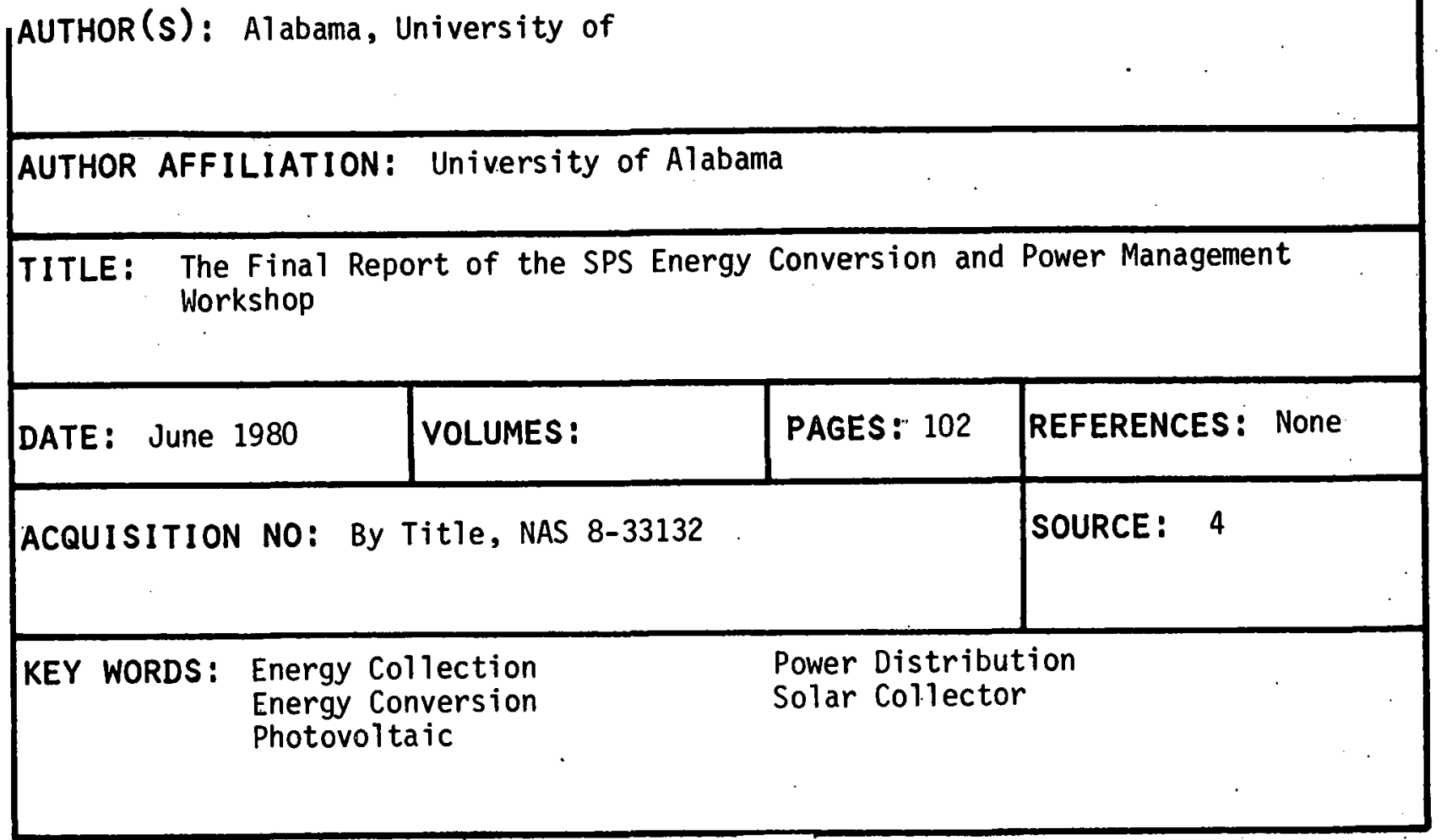

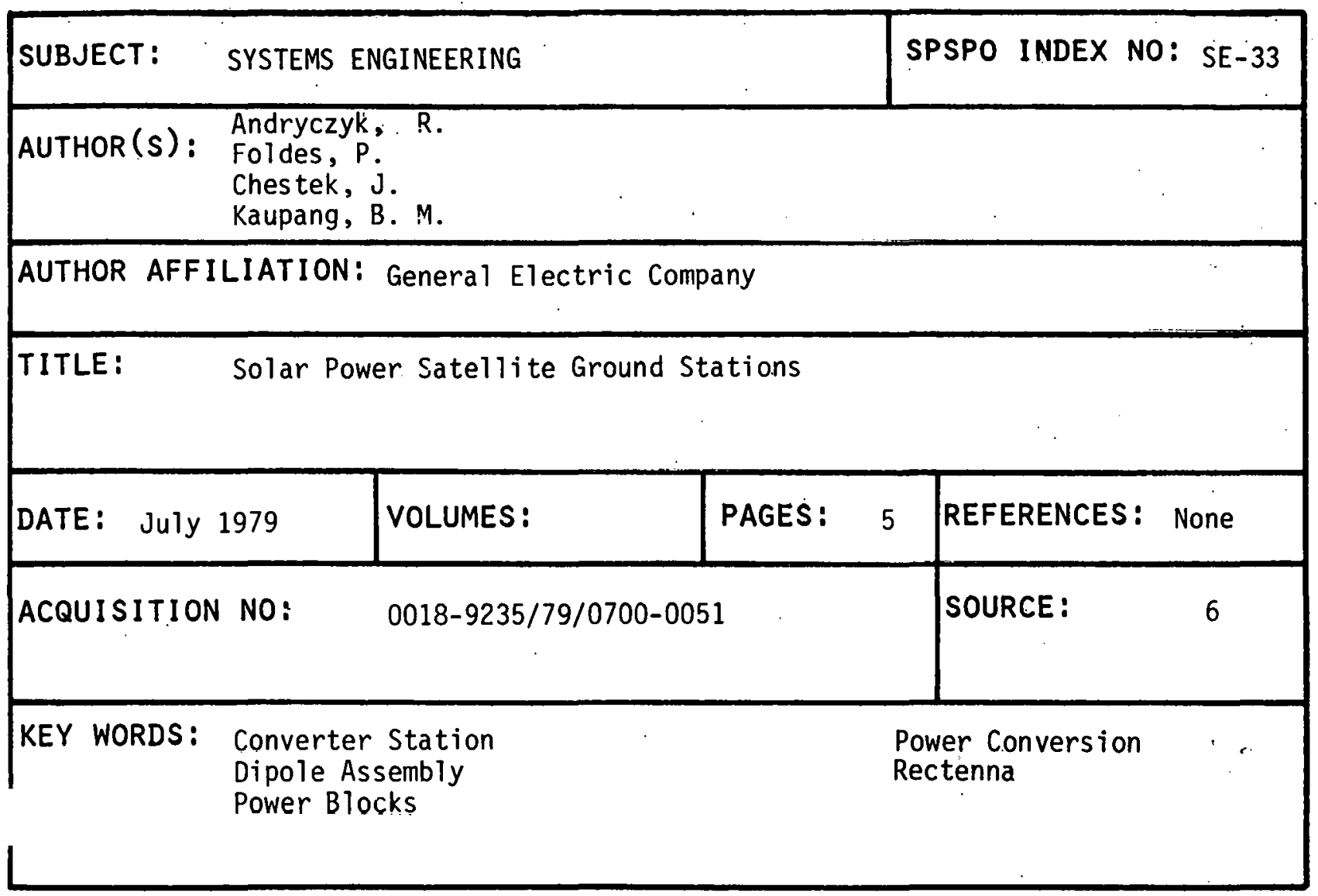


SUBJECT: SYSTEMS ENGIHEERING

AUTHOR(s): Argonne National Laboratory

AUTHOR AFFILIATION: Argonne National Laboratory

TITLE: Design Requirements for Orbit Maintenance of SPS Elements

DATE: November 1980

VOLUMES:

PAGES: 68

REFERENCES : 6

ACQUISITION NO: DOE/ER-0087

SOURCE: 5

KEY WORDS: Failure Analys is

Orbit Maintenance

Satellite Control

Space Transportation

\begin{tabular}{ll}
\hline SUBJECT: & SYSTEMS ENGIN \\
\hline AUTHOR (S): & $\begin{array}{l}\text { Bekey, I. } \\
\text { Blọçęr.W. W, }\end{array}$
\end{tabular}

AUTHOR AFFILIATION: Aerospace Corporation

TITLE: High Efficiency Low Cost Solar Cell Power

SPSPO INUEX NO: SE-36

\begin{tabular}{|c|l|l|l|}
\hline DATE: November 1978 & VOLUMES: & PAGES: 7 & REFERENCES: 12 \\
\hline ACQUISITION NO: & $\begin{array}{l}\text { By Title; Astronautics and } \\
\text { Aeronautics, Pp. 32-38 }\end{array}$ & SOURCE: \\
\hline KEY WORDS: & $\begin{array}{l}\text { Array Design } \\
\text { Cel1 Design } \\
\text { Concentrators }\end{array}$ & $\begin{array}{l}\text { Efficiency } \\
\text { Performance }\end{array}$ & \\
\hline
\end{tabular}




\begin{tabular}{|c|c|c|c|c|}
\hline \multicolumn{4}{|c|}{ SUBJECT: SYSTEMS ENGINEERING } & SPSPO INDEX NO: SE-15 \\
\hline $\operatorname{AUTHOR}(s): \quad B$ & \multicolumn{4}{|c|}{ Boeing Aerospace Company } \\
\hline \multicolumn{5}{|c|}{ AUTHOR AFFILIATION: Boeing Aerospace Company } \\
\hline TITLE: & \multicolumn{4}{|c|}{ Satellite Power System System Definition Stuidy, Parts I and II } \\
\hline DATE: Decembe & ber 1977 & VOLUMES: 7 & PAGES: 1317 & REFERENCES : None \\
\hline ACQUISITION & \multicolumn{3}{|c|}{$\begin{array}{l}\text { No: } \frac{1}{\text { DI80228-1,2,3,4,5,6,8 }} \text { NAS9-15196 } \\
\text { DRL T-1346 } \\
\text { DRD MA-664T }\end{array}$} & SOURCE: \\
\hline KEY WORDS: $\begin{array}{r}S \\
S \\
S\end{array}$ & \multicolumn{3}{|c|}{$\begin{array}{l}\text { Satellite Structure } \\
\text { Space Construction } \\
\text { Space Transportation }\end{array}$} & $\begin{array}{l}\text { System Uncertainties } \\
\text { Systems Definition } \\
\text { Transmission System }\end{array}$ \\
\hline
\end{tabular}

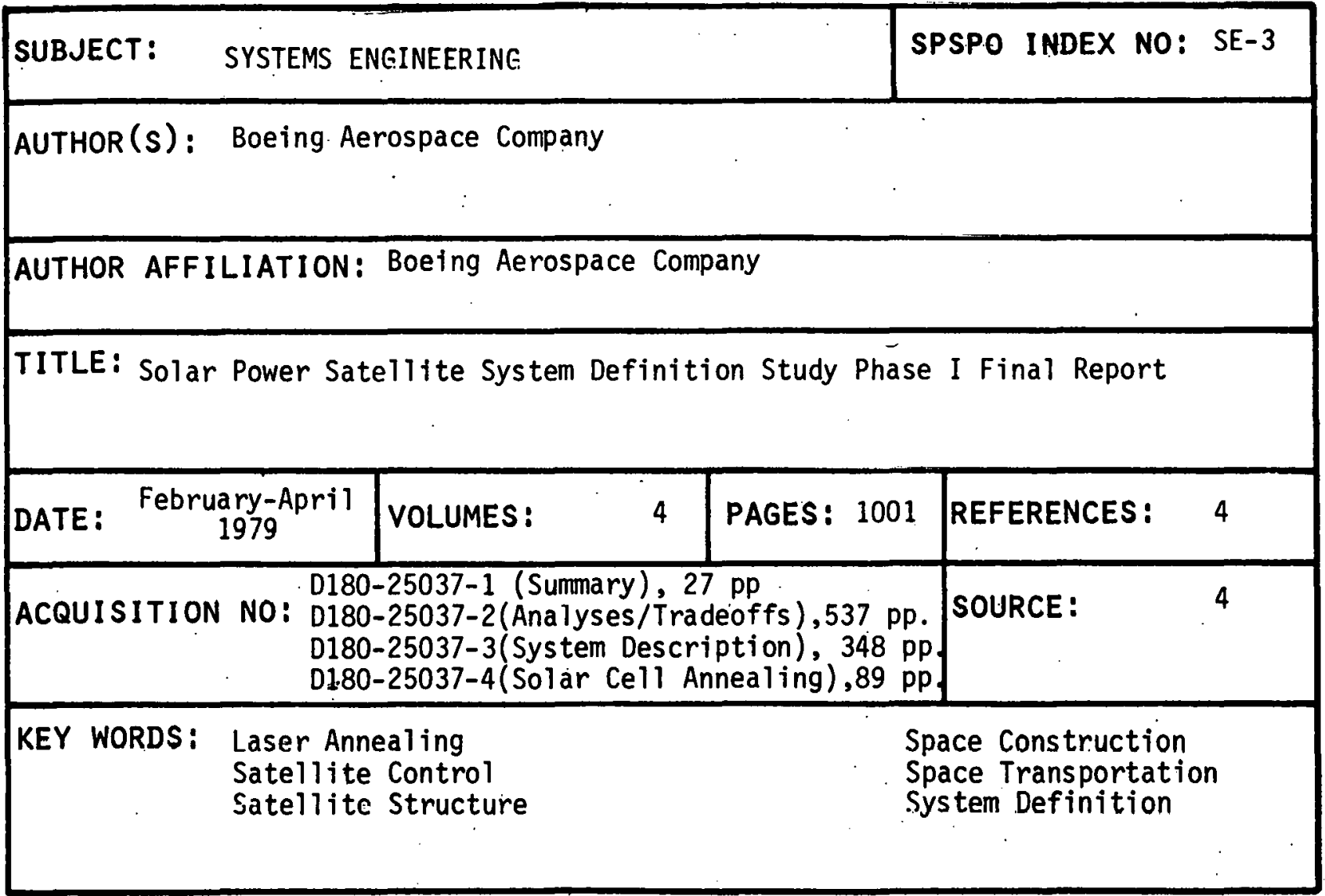


SUBJECT: SYSTEMS ENGINEER.ING

SPSPO INDEX NO: SE-4

1

AUTHOR(s): Boeing Aerospace Company

AUTHOR AFFILIATION: Boeing Aerospace Company

TITLE: Solar Power Satellite System Definition Study, Part III, Preferred Concept

\begin{tabular}{|l|l|l|l|}
\hline DATE: March 1978 & VOLUMES: & PAGES: 357 & REFERENCES: \\
\hline ACQUISITION NO: $\begin{array}{l}\text { NAS9-15196 } \\
\text { DRL T-1346 } \\
\text { DRD MA-664T } \\
\text { D 180-24071-1 }\end{array}$ & SOURCE: \\
\hline KEY WORDS: $\begin{array}{l}\text { Energy Collection } \\
\text { Energy Conversion } \\
\text { Ground Station }\end{array}$ & $\begin{array}{l}\text { Power Distribution } \\
\text { Satellite Structure } \\
\text { Transmission System }\end{array}$ \\
\hline
\end{tabular}

SUBJECT: SYSTEMS ENGINEERING

SPSPO INDEX NO: SE-24

AUTHOR(s): Boeing Aerospace Company

AUTHOR AFFILIATION: Boeing Aerospace Company

TITLE: Solar Power Satellite System Definition Study, Phase II

DATE: November 1979

VOLUMES : 4

PAGES: 1272

REFERENCES: 32

ACQUISITION NO:

D180-25461-1,2,3,4,

Vol. I, Summary, $37 \mathrm{pp}$.

Vol.II, Description, $340 \mathrm{pp}$.

KEY WORDS: Energy Collection

Energy Conversion

Ground Station
Vol.III, Synthesis, $599 \mathrm{pp}$.

\section{SOURCE :}

Vol.IV, Analysis, 296 pp.

Satellite Structure

Space Construction

Space Transportation 
SUBJECT: SYSTEMS ENGINEERING

AUTHOR(s): Bresters, A. R.

AUTHOR AFFILIATION: Hydronamic B. V.

TITLE: Study on Infrastructure Considerations for Microwave Energy Ground Receiving Stations (SPS - Offshore Rectenna Siting Study in WestEurope)

\begin{tabular}{|l|l|l|l|}
\hline DATE: November 1980 & VOLUMES: 2 & PAGES: 136 & REFERENCES: 3 \\
\hline
\end{tabular}

\begin{tabular}{ll|l}
\hline ACQUISITION NO: ESTEC & $4382 / 80 / \mathrm{NL} / \mathrm{PP}(\mathrm{SC})$ & SOURCE: \\
V. 1, Summary, $25 \mathrm{pp}$. & V.2, Final Report, $111 \mathrm{pp}$. &
\end{tabular}

\begin{tabular}{|c|c|}
\hline KEY WORDS: & $\begin{array}{l}\text { Antenna } \\
\text { Energy Collection } \\
\text { Ground Station }\end{array}$ \\
\hline
\end{tabular}

SUBJECT: SYSTEMS ENGINEERING

AUTHOR(s): Brown and Root Development, Inc.

AUTHOR AFFILIATION: Brown and Root Development, Inc.

TITLE: Offshore Space Center

\begin{tabular}{|c|c|c|c|c|}
\hline DATE : Octob & ber 1979 & VOLUMES: & PAGES: 43 & REFERENCES: None \\
\hline \multicolumn{4}{|c|}{ ACQUISITION NO: EF-0082 } & SOURCE: 4 \\
\hline \multicolumn{3}{|c|}{$\begin{aligned} \text { KEY WORDS: } & \text { Rectenna Alternatives } \\
& \text { Rectenna Constructability } \\
& \text { Rectenna Costs }\end{aligned}$} & \multicolumn{2}{|c|}{$\begin{array}{l}\text { Rectenna Desiǵn } \\
\text { Rectenna Maintainability } \\
\text { Rectenna Materials }\end{array}$} \\
\hline
\end{tabular}


AUTHOR(S): Chan, C. H.

AUTHOR AFFILIATION: Marshall Space Flight Center

TITLE: Pilot Signals for Large Active Retro-Directive Arrays

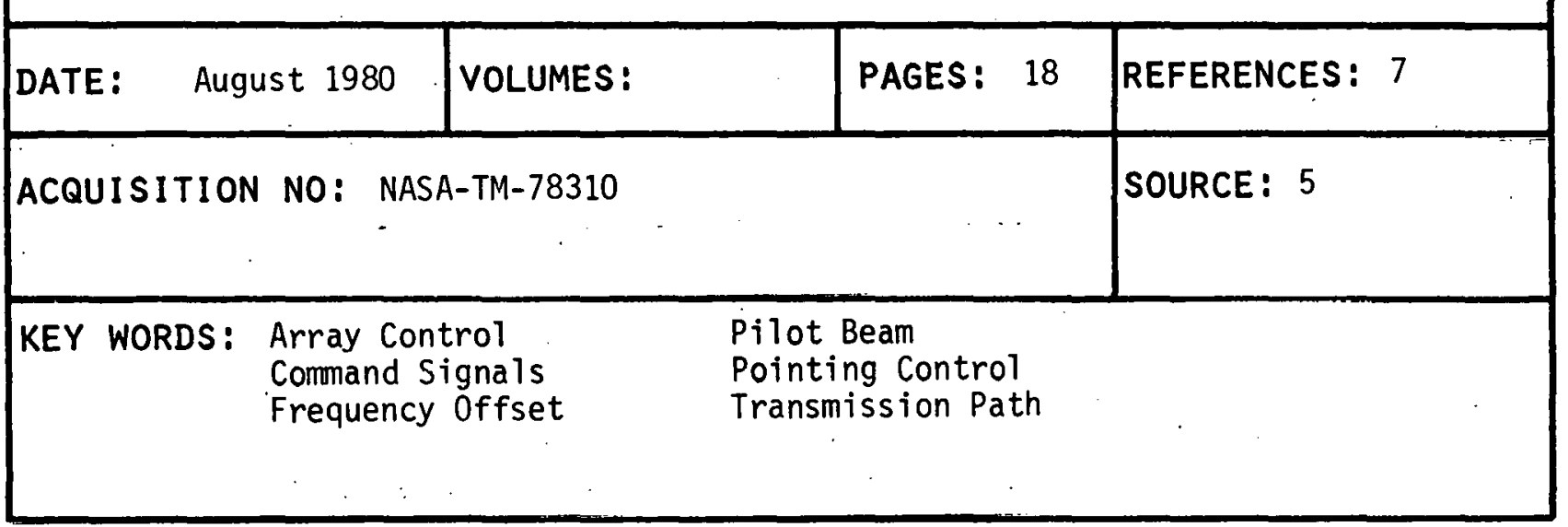

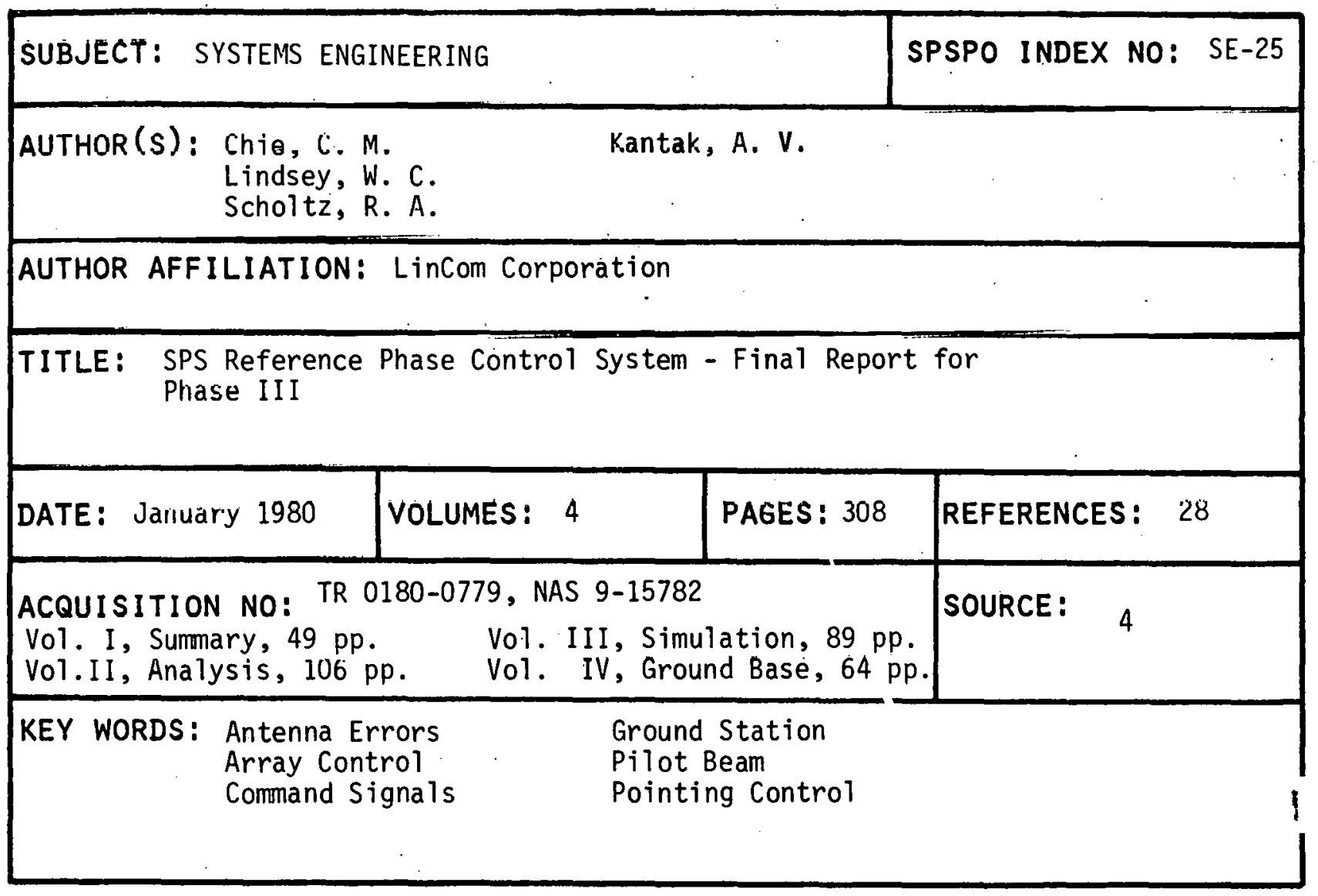


AUTHOR(s): Collins, P. Q.

AUTHOR AFF.ILIATION: Imperial College of Science and Technology (London)

TITLE: Feasibility of Siting SPS Rectennas Over the Sea

DATE : 1980

VOLUMES:

PAGES: 12

REFERENCES: 26

ACQUISITION NO:

0191-9067/80/010133-12

SOURCE : $\quad 33$

Space Solar Power Review, Vol. 1, pp. 133-144

KEY WORDS: Ground Station

Offshore Rectenna

Rectenna Alternatives

Rectenna Constructability

Rectenna Lesign

Rectenna Maintainability

\begin{tabular}{|c|c|c|c|c|c|}
\hline \multicolumn{4}{|c|}{ SUBJECT: SYSTEMS ENGINEERING } & \multicolumn{2}{|c|}{ SPSPO INDEX NO: SE-34 } \\
\hline AUTHOR $(s):$ & \multicolumn{5}{|c|}{$\begin{array}{l}\text { Donalek, P. J. } \\
\text { Whysong, J. L. }\end{array}$} \\
\hline \multicolumn{6}{|c|}{ AUTHOR AFFILIATION: Harza Engineering Company } \\
\hline TITLE: & \multicolumn{5}{|c|}{ Utility Interface Requirements for a Solar Power System } \\
\hline DATE : Septem & ber 1978 & VOLUMES: & PAGES: 57 & REFERENCES : & 8 \\
\hline ACQUISITION & NO: & \multicolumn{2}{|c|}{$D O E / E R-0032$} & SOURCE: & 5 \\
\hline KEY WORDS : & \multicolumn{3}{|c|}{$\begin{array}{l}\text { Load Estimates } \\
\text { Power Blocks } \\
\text { Rectenna }\end{array}$} & \multicolumn{2}{|l|}{$\begin{array}{l}\text { Reliability } \\
\text { Stability } \\
\text { System Capacity }\end{array}$} \\
\hline
\end{tabular}


SUBJECT: SYSTEMS ENGINEERING

AUTHOR(s): Dunn, B. D.

Collins, D. S.

AUTHOR AFFILIATION: European Space Agency

TITLE: Materials Investigation for a Failed Spacecraft Antenna

\begin{tabular}{|l|l|l|l|}
\hline DATE: 1978 & VOLUMES: & PAGES: 13 & REFERENCES: 5 \\
\hline ACQUISITION NO: $\begin{array}{l}\text { ESA Journal 78/3, Vol 2. } \\
\text { pp. 223-235 }\end{array}$ & SOURCE: \\
\hline KEY WORDS: $\begin{array}{l}\text { Antenna } \\
\text { Debonding } \\
\text { Failure Analysis }\end{array}$ & $\begin{array}{l}\text { Fatigue } \\
\text { Vibration Test }\end{array}$ \\
\hline
\end{tabular}

SUBJECT: SYSTEMS ENGINEERING

SPSPO. INDEX NO: SE-11

AUTHOR(S): Energy, U.S. Department of

National Aeronautics and Space Administration

AUTHOR AFFILIATION: U.S. Department of Energy

National Aeronautics and Space Administration

TITLE: Satellite Power System Concept Development and Evaluation Program Reference System Report

\begin{tabular}{|l|l|l|}
\hline DATE: Octuber 1978 & VOLUMES: & PAGES: $310 \begin{array}{l}\text { REFERENCES: } \\
\text { Bibliography }\end{array}$ \\
\hline ACQUISITION NO: & SOE/ER-0023 & \\
\hline KEY WORDS: $\begin{array}{l}\text { Power Distribution } \\
\text { Satellite Control } \\
\text { Satellite Structure }\end{array}$ & $\begin{array}{l}\text { Space Transportation } \\
\text { System Definition } \\
\text { Transmission System }\end{array}$ \\
\hline
\end{tabular}




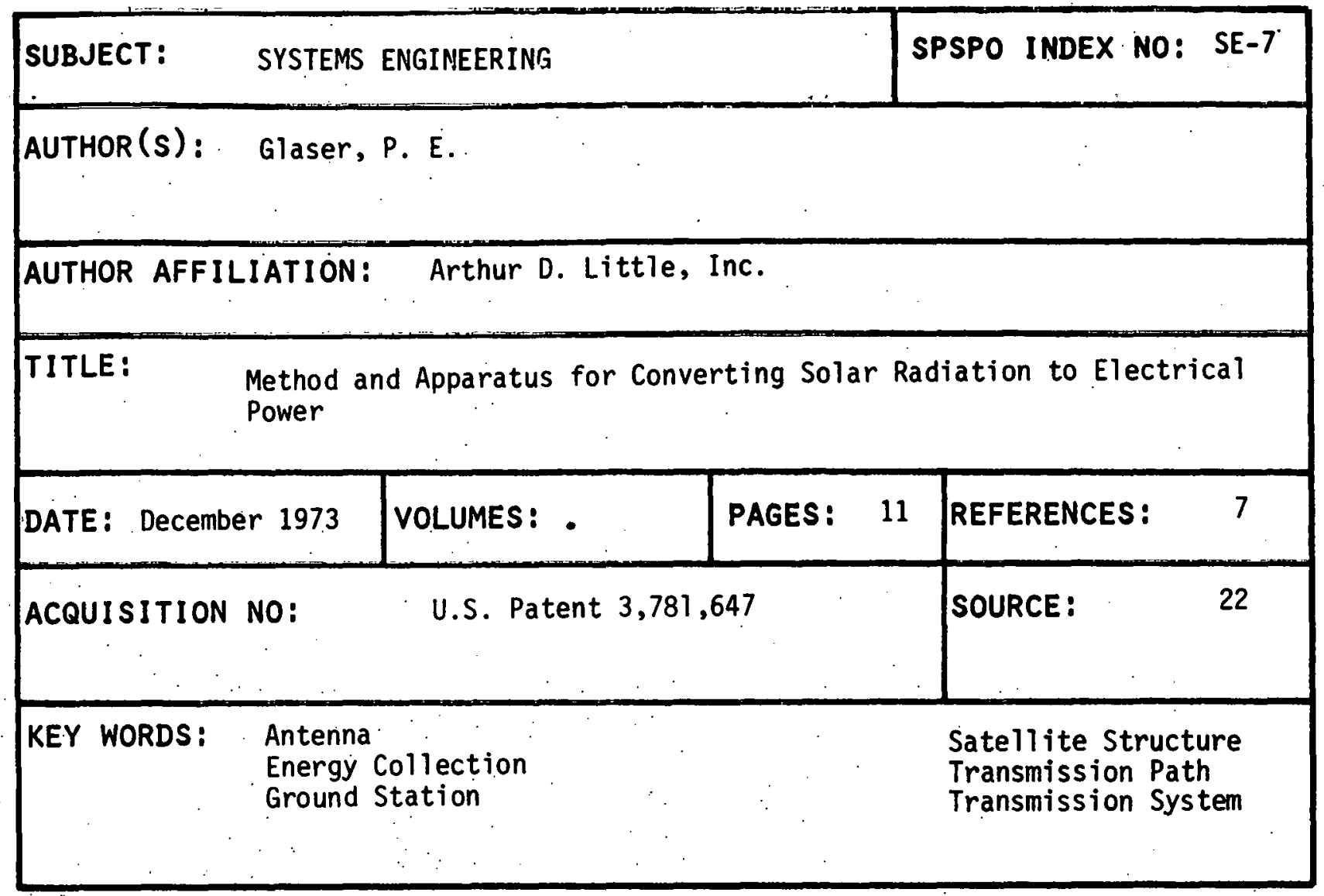

\begin{tabular}{|c|c|c|c|c|}
\hline SUBJECT : & SYSTEMS ENGINEERING & $\cdot$ & \multicolumn{2}{|c|}{ PSPO INDEX NO: SE-35 } \\
\hline $\begin{array}{r}\operatorname{AUTHOR}(\mathrm{S}): \\
\cdot\end{array}$ & \multicolumn{4}{|l|}{$\begin{array}{l}\text { Glaser, } P . E \text {. } \\
\text { Almgren, D. W. } \\
\text { Csigi, K. } \\
\text { Gaudet, A. D. }\end{array}$} \\
\hline \multicolumn{5}{|c|}{ AUTHOR AFFILIATION: Arthur D. Little, Inc. } \\
\hline TITLE : & \multicolumn{4}{|c|}{$\begin{array}{l}\text { Evaluation of Solar Cells and Arrays for Potential Solar Povier } \\
\text { Satellite Applications }\end{array}$} \\
\hline DATE: March & VOLUMES: & PAGES: 75 & REFERENCES : & 42 \\
\hline ACQUISITION & NASA $9-15$ & & SOURCE : & 4 \\
\hline $\begin{array}{c}\text { KEY WORDS: } \\
\vdots\end{array}$ & \multicolumn{4}{|c|}{$\begin{array}{ll}\text { Array Design } & \text { Federal Programs } \\
\text { Cell Design } & \text { Industrial Capacity } \\
\text { Economics } & \text { Manufacturing Process }\end{array}$} \\
\hline
\end{tabular}


SUBJECT: SYSTEMS ENGINEERING

SPSPO INDEX NO: SE-13

$\begin{array}{llll}\text { AUTHOR (S): } & \begin{array}{l}\text { Gorman, D. } \\ \text { Grant, C. } \\ \text { Kyrias, G. }\end{array} & \begin{array}{l}\text { Lord, C. } \\ \text { Rombach, J. Salis, M. }\end{array} & \begin{array}{l}\text { Skidmore, R. } \\ \text { Thomas, R. }\end{array}\end{array}$

AUTHOR AFFILIATION:

Martin Marietta Corporation

TITLE: Orbital Assembly and Maintenance Study

\begin{tabular}{|l|l|l|l|}
\hline DATE: August 1975 & VOLUMES: & PAGES: 25 & REFERENCES: None \\
\hline ACQUISITION NO: & $\begin{array}{l}\text { MCR-75-319 } \\
\text { NAS9-14319 }\end{array}$ & SOURCE: \\
\hline
\end{tabular}

KEY WORDS: Antenna

Satellite Maintainability

Transmission System

Space Construction

SUBJECT: SYSTEMS ENGINEERING

SPSPO. INDEX NO: SE-10

AUTHOR(s): Grumman Aerospace Corporation

AUTHOR AFFILIATION: Gruman Aerospace Corporation

TITLE: Orbital Construction Demonstration Study

\begin{tabular}{|c|c|c|c|c|c|}
\hline DATE: June 1 & 1977 & & VOLUMES: 3 & PAGES: 551 & REFERENCES : 104 \\
\hline ACQUISITION & NO: & \multicolumn{3}{|c|}{$\begin{array}{l}\text { NSS-OC-RPO-12 } \\
\text { V.1 - Summary ( } 61 \text { pp.) } \\
\text { V.2 - Technical ( } 339 \text { pp.) } \\
\text { V.3 - Requirements (91 pp.) }\end{array}$} & SOURCE: 4 \\
\hline KEY WORDS: & $\begin{array}{l}\text { Ante } \\
\text { Sate } \\
\text { Sate }\end{array}$ & $\begin{array}{l}n n a \\
11 \text { ite } \\
11 \text { ite }\end{array}$ & $\begin{array}{l}\text { Control } \\
\text { Structure }\end{array}$ & & $\begin{array}{l}\text { ce Automation } \\
\text { ce Construction }\end{array}$ \\
\hline
\end{tabular}


AUTHOR(s): Guttman, R. J. (Editor)

AUTHOR AFFILIATION: Rensselaer Polytechnic Institute

TITLE :

Solar Power Satellites Design Considerations for Solar Collection and Conversion, Power Beam Phase Control and Rectenna Subsystems

\begin{tabular}{|l|l|l|l|}
\hline DATE: June 1978 & VOLUMES: 1 & PAGES: 145 & REFERENCES: 50 \\
\hline ACQUISITION NO: NAS9-15453 & SOURCE: \\
\hline KEY WORDS: $\begin{array}{l}\text { Antenna } \\
\begin{array}{l}\text { Energy Collection } \\
\text { Energy Conversion }\end{array}\end{array}$ & $\begin{array}{l}\text { Pilot Beam } \\
\text { Rectenna Design } \\
\text { Transmission System }\end{array}$ \\
\hline
\end{tabular}

SUBJECT: $\quad$ SYSTEMS ENGINEERING

AỤTHOR (s): $\begin{array}{r}\text { Guttmanin, R. J. } \\ \text { Borrego, J. M. }\end{array}$

AUTHOR AFFILIATION: Rensselaer Polytechnic Institute

TITLE :

Solar Power Satellite Rectenna Design Study: Directional Receiving Elements and Parallel-Series Combining Analys is

DATE: December 1978

VOLUMES:

PAGES: 116

REFERENCES : 21

ACQUISITION NO:

NAS9-15453

SOURCE :

4

KEY WORDS: Ground Station

Power Distribution

Rectenna Alternatives

Rectenna Costs

Rectenna Design 
AUTHOR (s): Hanley, G, M.

AUTHOR AFFILIATION: Rockweil International

TITLE: - Satellite Power System (SPS) Concept Definition Study Key Issue Identification and Assessment

\begin{tabular}{|c|c|c|c|c|}
\hline DATEI & 1977 & VOLUMES : & PAGES: 34 & REFERENCES : None \\
\hline ACQUISITION & NO: & SD 77-AP-0103-2 & & SOURCE: \\
\hline KEY WORDS : & $\begin{array}{l}\text { Energy } \\
\text { Groun } \\
\text { Sate } 1\end{array}$ & $\begin{array}{l}\text { nversion } \\
\text { ation } \\
\text { Maintainability }\end{array}$ & & $\begin{array}{l}\text { Space Construction } \\
\text { Space Transportation } \\
\text { Transmission System }\end{array}$ \\
\hline
\end{tabular}

SUBJECT: SYSTEMS ENGINEERING

SPSPO IÑDEX NO: SE-8

$\operatorname{AUTHOR}(s): \quad H a n l e y, G . M$.

AUTHOR AFFILIATION: Rockwell International

TITLE :

Satellite Power Systems (SPS) Concept Definition Study Final Report

\begin{tabular}{ll|l|l|l}
\hline DATE: & April 1978 & VOLUMES: 7 & PAGES: 1323 & REFERENCES: None \\
\hline
\end{tabular}

SD 78-AP-0023 V.2-Requirements(86 pp) V.6-Technology

ACQUISITION NO: V.3-Evolution(289 pp.)

V.7-Summary (77 pp.) V.4-Design(188 pp.)

(129 pp.) SOURCE:

4

V.5-Transportation and Operations (168 pp.)

V.7-Economics

(299 pp.)

KEY WORDS: Energy Conversion

Ground Station

Power Distribution

Satellite Structure

Space Transportation

Transmission System 
SUBJECT: $\quad$ SYSTEMS ENGINEERING

AUTHOR (s): Hanley, G. M.

AUTHOR AFFILIATION: Rockwell International

TITLE: Satellite Power Systems (SPS) Concept Definition Study - Point Design Definition Update

\begin{tabular}{|l|l|l|l}
\hline DATE: July 1978 & VOLUMES: & PAGES: 107 & REFERENCES: None \\
\hline
\end{tabular}

ACQUISITION NO: SD78-AP-0113

NAS8-32475

SOURCE :

DPD 558

KEY WORDS: Ground Station.

Power Distribution

Satellite Control

Satellite Materials

Satellite Structure

Transmission System

SUBJECT: SYSTEMS ENGINEERING

SPSPO INDEX NO: SE-16

$\operatorname{AUTHOR}(s): \quad H a n l e y$, G. M.

AUTHOR AFFILIATION: Rockwell International

TIITLE: $\quad$ Satellite Power Systems (SPS) Concept Definition Study Final Report

\begin{tabular}{|l|l|l|lc|}
\hline DATE: March 1979 & VOLUMES: 9 & PAGES: 1336 & REFERENCES: 3 \\
\hline ACQUISITION NO: $\begin{array}{l}\text { SSD 79-0010-1 through }-7 \\
\text { NAS8-32475 } \\
\text { DPD558 MA-04 }\end{array}$ & SOURCE: & 4 \\
\hline
\end{tabular}

KEY WORDS: Ground Station

Satellite Structure

Space Construction

Space Transportation

Systems Definition

Transmission System 
AUTHOR(s): Huie, H. H.

AUTHOR AFFILIATION: Marshall Space Flight Center

TITLE: $\quad$ Test of Concentrator Solar Array Model for SEPS

DATE : May 1979

VOLUMES :

PAGES : 7.8

REFERENCES: None

SOURCE: 4

ACQUISITION NO: NASA TM-78230

Photovoltaics

KEY WORDS: Concentrators

Energy Collection

Energy Conversion

SUBJECT: SYSTEMS ENGINEERING

SPSPO INDEX NO: SE-18

AUTHOR(s): Hung, J. C.

Peebles, P. Z.

AUTHOR AFFILIATIUN: University of Iennéssee

TITLE: Accuracy Analys is of Pointing Control System of Solar Power Satellite

\begin{tabular}{|l|l|l|l|}
\hline DATE: December 1978 & VOLUMES: & PAGES: 67 & REFERENCES: None \\
\hline ACQUISITION NO: & NAS8-33065 & SOURCE: \\
\hline KEY WORDS: $\begin{array}{l}\text { Antenna Errors } \\
\text { Array Control } \\
\text { Command Signals }\end{array}$ & $\begin{array}{l}\text { Pilot Beam } \\
\text { Pointing Control } \\
\text { Satelite Structure }\end{array}$
\end{tabular}




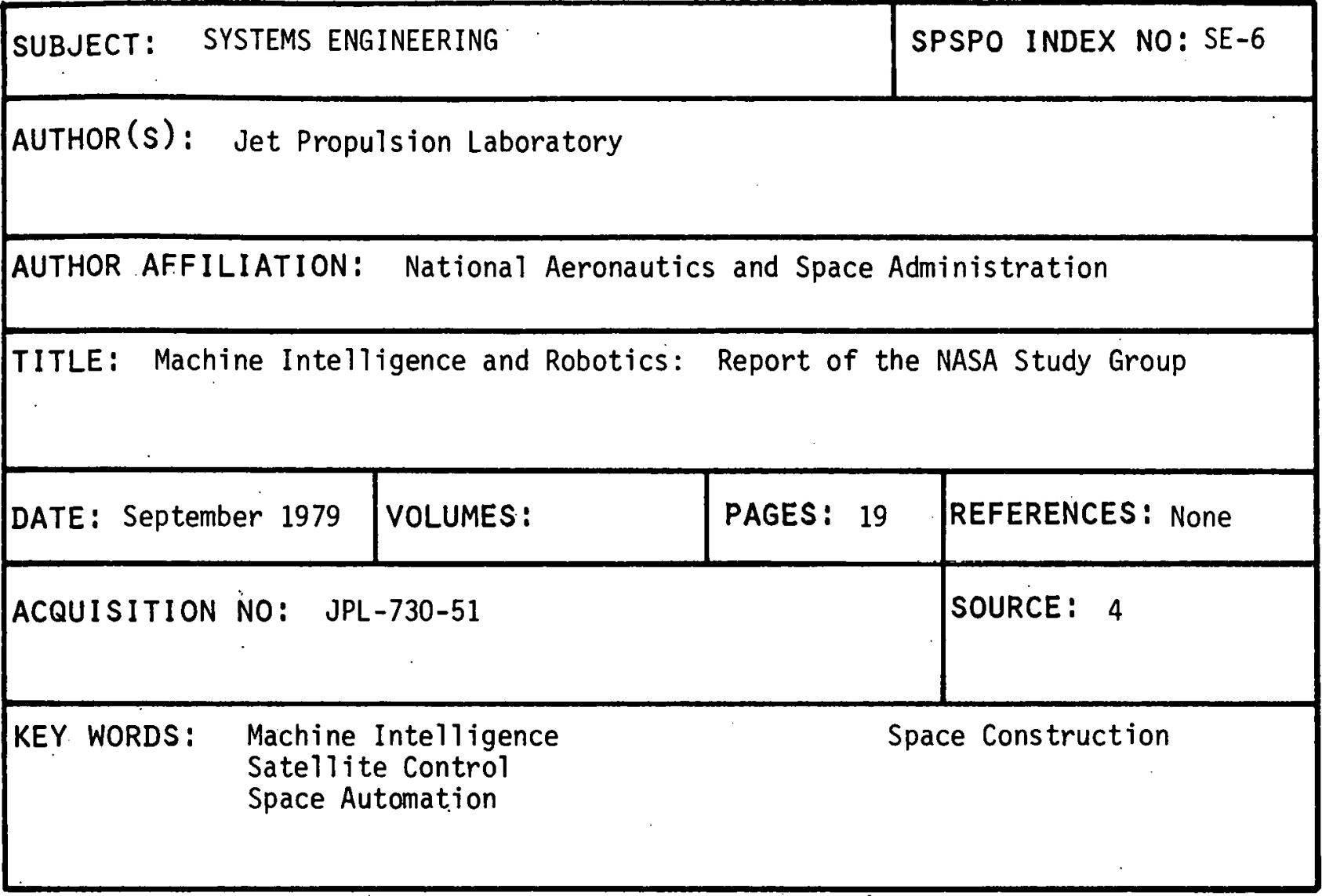

\section{SUBJECT: SYSTEMS ENGINEERING}

SPSPO INDEX. NO: SE-5

AUTHOR (S): Johnson, R. W.

AUTHOR AFFILIATION: Grumman Aerospace Corporation

TITLE: Solar Power Satellite: Putting It Together

\begin{tabular}{|l|l|l|l|}
\hline DATE: September 1979 & VOLUMES: & PAGES: 4 & REFERENCES: None \\
\hline ACQUIS ITION NO: $0018-9235 / 79 / 0900-0037$ & & SOURCE: \\
\hline
\end{tabular}

KEY WORDS: Orbit Position

Satellite Materials

Satellite Structure

Space Construction

Space Transportation 
AUTHOR(s): Lindsay, T: A.

Nalos, E. J.

AUTHOR AFFILIATION: Boeing Aerospace Company

TITLE: SPS Fiber Optic Link Assessment

\begin{tabular}{|c|c|c|c|c|}
\hline DATE: January 1980 & VOLUMES: & PAGES: 94 & REFERENCES : & 16 \\
\hline ACQUISITION NO: & $\begin{array}{l}80-25888-1 \\
9-15636 A\end{array}$ & & SOURCE : & 4 \\
\hline
\end{tabular}

KEY WORDS: Array Control

Pointing Control

Command Signals

Fiber Optics

SUBJECT: SYSTEMS ENGINEERING

SPSPO INDEX NO: SE-2

AUTHOR (s): Raytheon Company

AUTHOR AFFILIATION: Raytheon Company

TITLE: Solar Power Satellite (SPS) Pilot Beam and Communication Link Suhsyst.em investigationn Repnrt---Phase I

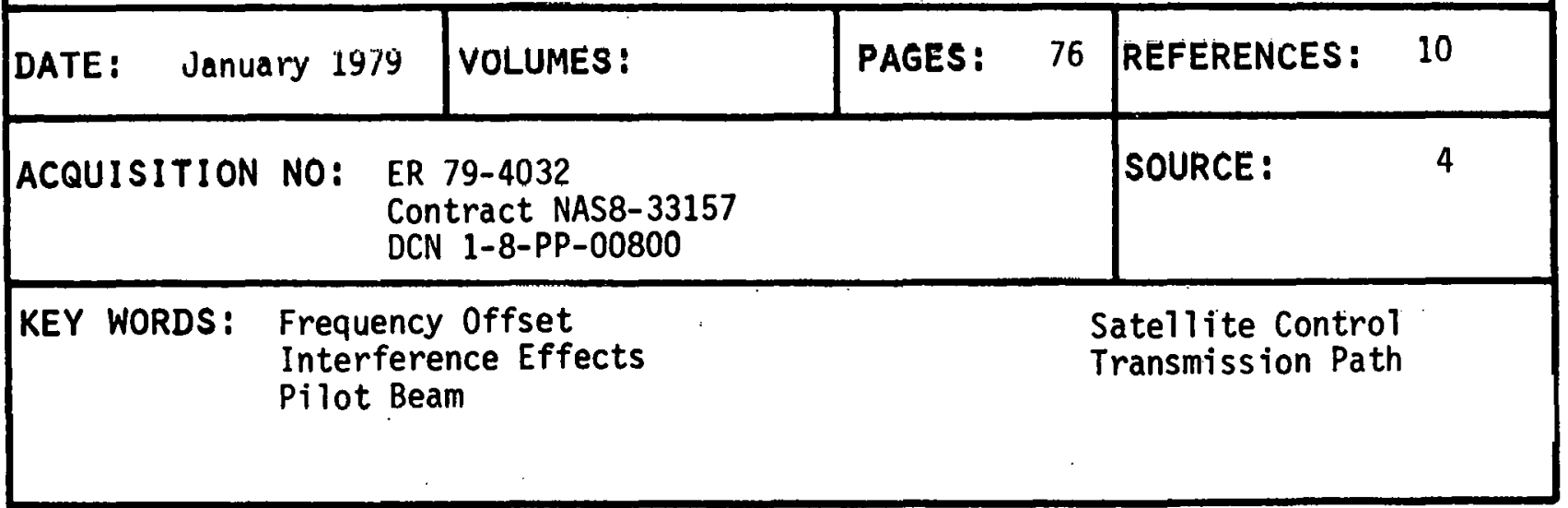


AUTHOR(s): Reiff, P. H.

Freeman, J. W.

Cooke, D. L.

AUTHOR AFFILIATION: Rice University

TITLE: Environmental Protection of the Solar Power Satellite

DATE: 1979

VOLUMES:

PAGES: 23

REFERENCES： 25

ACQUISITION NO: By Title, Progress in Astronautics and Aeronautics, Vol. 71

SOURCE : 2

KEY WORDS: Electrostatic Protecion

Satellite Materials

Satellite Structure

SUBJECT: SYSTEMS ENGINEERING

AUTHOR(S): Rice University

AUTHOR AFFILIATION: Rice University

TITLE: Solar Power Satellite Offshore Rectenna Study

\begin{tabular}{|c|c|c|c|}
\hline DATE: November 1980 & VOLUMES: & PAGES: 257. & REFERENCES: 6 \\
\hline ACQUISITION NO: & $\begin{array}{l}\text { A CR } 3348 \\
8-33023\end{array}$ & & SOURCE: 4 \\
\hline
\end{tabular}

KEY WORDS: Antenna

Energy Collection

Ground Station

Offshore Rectenna

Rectenna Costs

Rectenna Design 
AUTHOR(s): Rice University.

AUTHOR AFFILIATION: Rice University

TITLE: Electrostatic Protection of the Solar Power Satellite and Rectenna;

Part I: Protection of the Solar Power Satellite

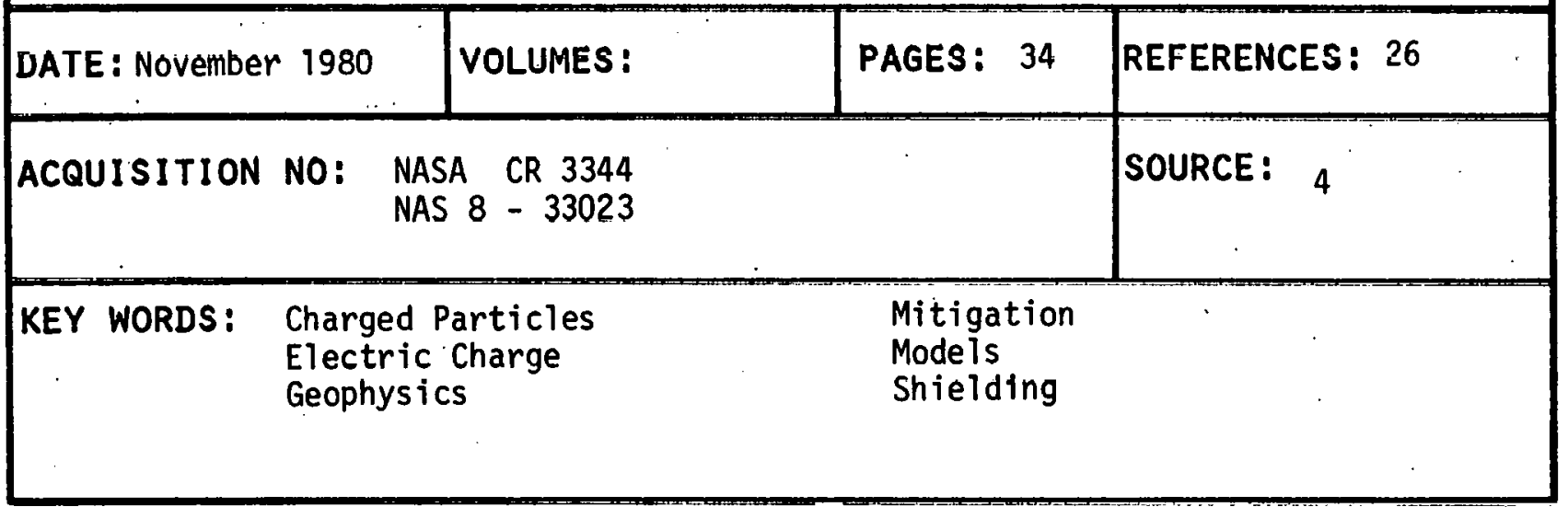

SUBJECT: SYSTEMS ENGINEERING SPSPO INDEX NO: SE-12

AUTHOR(S) I Taylor, R. B.

AUTHOR AFFILIATION: Bovay Engineers, Inc.

TITLE: Feasibility Study for Various Approaches to the Structural Design and Arrangement of the Ground Rectenna for the Propused Salellite

\begin{tabular}{|l|l|l|l|}
\hline DATE: May 1977 & VOLUMES: & PAGES: 72 & REFERENCES: None \\
\hline ACQUISITION NO: & Contract NAS9-15280 & SOURCE: \\
\hline KEY WORDS: & $\begin{array}{l}\text { Rectenna Constructability } \\
\text { Rectenna Costs } \\
\text { Rectenna Design }\end{array}$ & $\begin{array}{l}\text { Rectenna Heat } \\
\text { Rectenna Maintainability } \\
\text { Rectenna Materials }\end{array}$ \\
\hline
\end{tabular}


SUBJECT: SYSTEMS ENGINEERING

SPSPO INDEX NO: SE-29

nJTHOR(s): Tennessee, University of

AUTHOR AFFILIATION: University of Tennessee

TITLE: Satellite Power System (SPS) Antenna Pointing Control

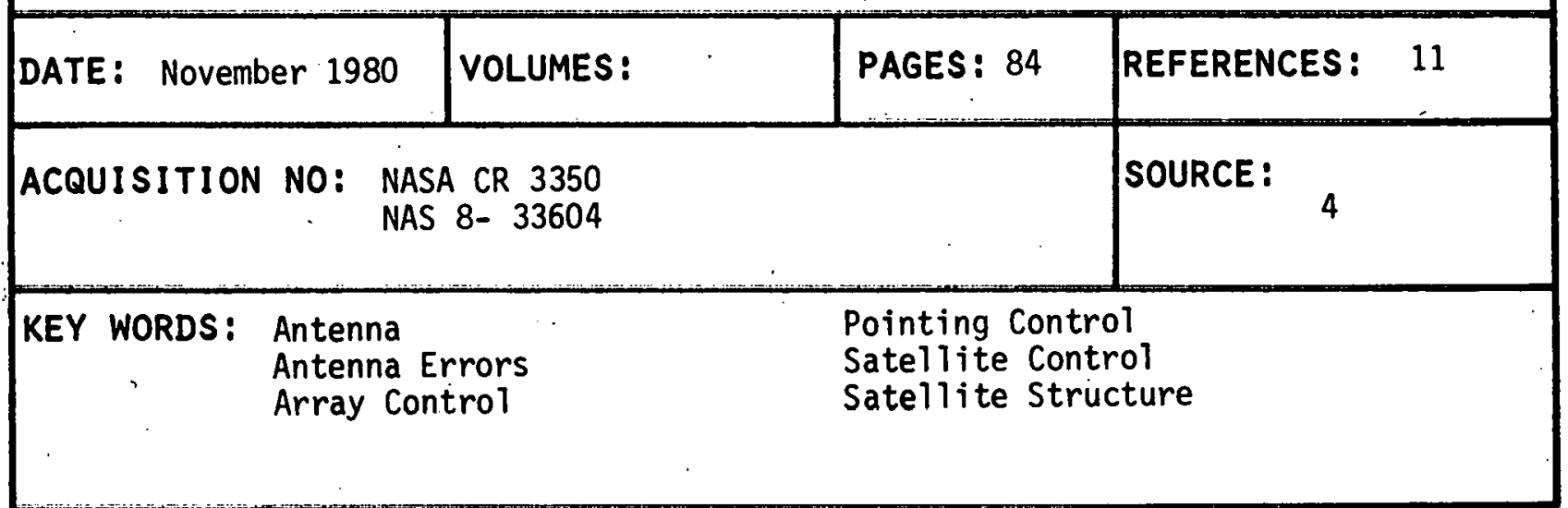




\section{THIS PAGE WAS INTENTIONALLY LEFT BLANK}




\section{SOCIAL ISSUES \\ (CATEGORY SI)}

Reports and other documents in this category cover a wide range of social issues and questions about the SPS concept, and describe the elements of the SPS Social Assessment CDEP and its results. The social implications of all key issues are described.

KEY WORDS:

Building Uses

Commercial Market

Damage Liability

Decentralization

Demographics

Economics

Energy Analys is

Energy Options

Environmental Concerins

Financial Regulation

Industry

Institutional Concerns

International Concerns

Internationa? Interest

Key Issues

Land Use

Liability

Microwave Standards

Military Issues

Ownership

Performance

Population

Power Conversion

Power Regulation.

Pricing

Public Acceptance

Public Outreach
PAGE NUMBER:

211

211

$201,205,210$

$200,202,204,205,206,207,208,214$

$199,200,206$

$197,199,200,202,203,204,205,206$

$207,208,209,210,211,213,214$

2.11

$203,206,207,211,213$

$198,199,201,202,205,207,212,213$

$200,202,203,204,209$

199,204

$199,200,202,204,205,210,212,213,214$

$198,199,201,203,205,207,208,209,210$

212,213

$203,204,214$

$198,201,207$

$199,201,205,209,214$

197

201

$198,201,205,208,212$

197

211

$199,203,206$

197

$200,202,203,204,208,209,211$

197

$198,199,206,207,208,210,211,213,214$

$198,201,209,210,213$ 
(Sociat Issues

Category SI Continued)

KEY WORDS:

Radio Spectrum

Regulation

Reliability

Resource Use

Satellite Vulnerability

Space Law

Stability

Student Interest

System Capacity

Systém Storage

Terrestrial Solar

Western Europe
PAGE NUMBER:

201, 107

211

197

199, 202, 203, 206, 207

$198,208,212$

$201,207,208$

197

$205,209,210$

197

197

$200,204,206,214$

214

RELATED CATEGORIES:

Biologic Effects (BE)

Energy Alternatives and Policy (EA)

Environmental Effects (EE)

Economics and Finances (EF)

Power Generation and Transmission (PT)

Resources and Materials (RM)

Systems Engineering (SE) 
AUTHOR(S): Arthur D. Little, Inc.

AUTHOR AFFILIATION: Arthur D. Little, Inc.

TITLE: $\quad$ Impacts and Benefits of a Satellite Power System on the Electric Utility Industry

DATE: JuTy 1977

VOLUMES:

PAGES : 128

REFERENCES: None

ACQUISITION NO: $\quad$ C-80020

JPL 954639

SOURCE : $\quad 4$

KEY WORDS: Liability

Ownership

Reliability

Pricing

Stability

SUBJECT: $\quad$ SOCIAL ISSUES

SPSPO INDEX NO: SI-35

AUTHOR(s): Asbury, J. G.

Mueller, R. 0.

AUTHOR AFFILIATION: Argonne National Laboratory

TITLE: Solar Energy and Electric Utilities: Should They Be Interfaced?

\begin{tabular}{|l|l|l|l|}
\hline DATE: $\quad$ February 1977 & VOLUMES: & PAGES: 6 & REFERENCES: 16 \\
\hline ACQUISITION NO: By Title; Science, Vol. 195, pp.445-450 & SOURCE: 7 \\
\hline KEY WORDS: $\begin{array}{l}\text { Economics } \\
\text { Ownership } \\
\text { Power Conversion }\end{array}$ & $\begin{array}{l}\text { Pricing } \\
\text { System Capacity } \\
\text { System Storage }\end{array}$ \\
\hline
\end{tabular}


SUBJECT :

SOCIAL ISSUES

AUTHOR(s): Bachrach, A.

AUTHOR AFFILIATION: Environmental/Resources Group

TITLE: $\quad$ Satellite Power System (SPS) Public Acceptance

DATE: Uctober 1978

ACEQUISITION NO: $\quad H C P / R-4024-04$

KEY WORDS: Environmental Concerns

International Concerns

Key Issues

\begin{tabular}{|l|l|}
\hline VOLUMES: & PAGES: 78. \\
\hline
\end{tabular}

REFERENCES : 186

SOURCE: $\quad 5$

\begin{tabular}{|l|l|}
\hline SUBJECT: SOCIAL ISSUES & SPSPO INDEX NO: SI-11 \\
\hline
\end{tabular}

AUTHOR(s): Bain, C.N.

AUTHOR AFFILIATION:

PRC Energy Analysis Company

TITLE :

Satellite Power System (SPS) Military Implications

DATE : October 1978

VOLUMES:

ACQUISITION NO: $\quad$ HCP/R-4024-11

KEY WORDS: International Concerns

Military Issues

Satellite Vuinerability
Public Acceptance

Public Outreach 


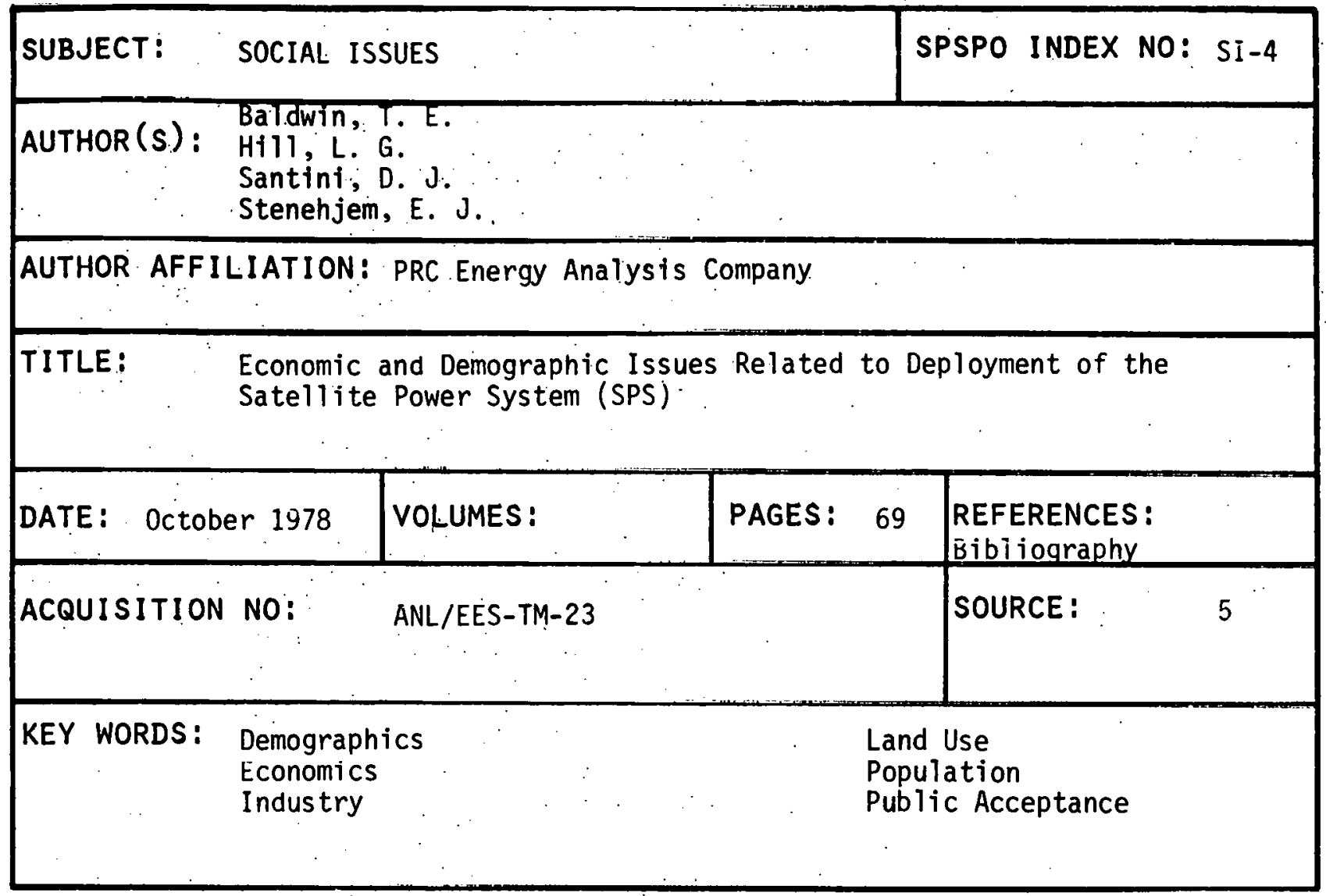

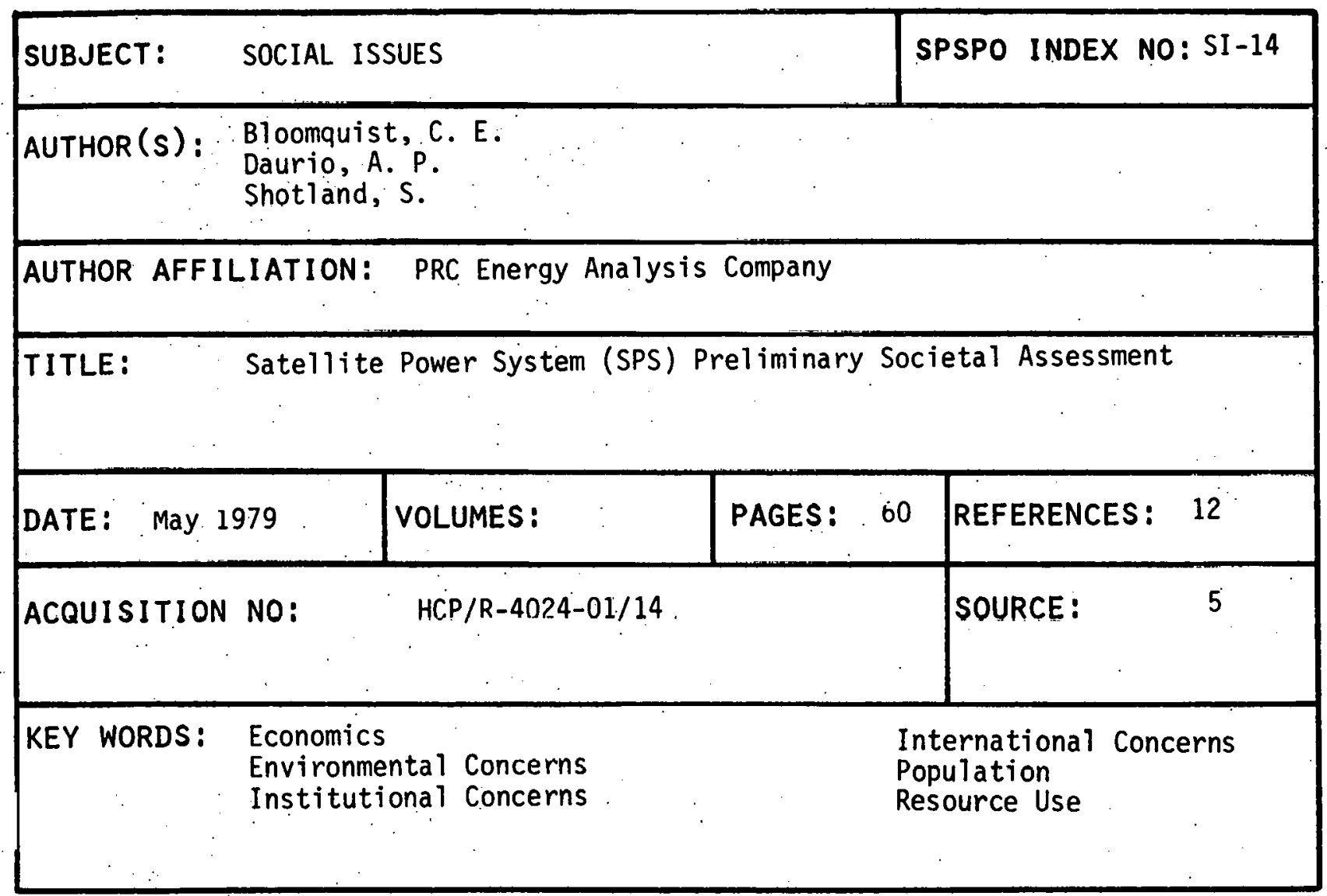


SUBJECT: SOCIAL ISSUES

$\operatorname{AUTHOR}(s)$ : Bohn, J. G.

Patmore, J.W.

Zaininger, H.W.

AUTHOR AFFILIATION: Systems Control, Inc.

TITLE: Satellite Power System: Utility Impact Study

DATE: September 1980

VOLUMES:

PAGES: 72

REFERENCES : 37

ACQUISITION NO: EPRI AP-1548

TPS $79-752$

SOURCE : 26

\begin{aligned} \hline KEY WORDS: & Economics \\ & Financial Regulation \\ & Institutional Concerns \end{aligned}

Power Regulation

SUBJECT: SOCIAL ISSUES

SPSPO INDEX NO: SI-27

AUTHOR (s): (1) Bronfman, B, H.

(1) Carnes, S. A.

(1) Schweitzer, $M$.

(1) Peelle, E.

(2) Enk, G.

AUTHOR AFFILIATION: (1) Oak Ridge National Laboratory

(2) Institute on Man and Science

TITLE: The Decentralized Solar Energy Technology Assessment Program: Review of Activities

DATE: May 1980

VOLUMES :

PAGES: 104

REFERENCES: 44

ACQUISITION NO: ORNL/TM-7189

SOURCE: 13

KEY WORDS: Decentralization

Demographics

Institutional. Concerns

Terrestrial Solar 
SUBJECT: SOCIAL ISSUES

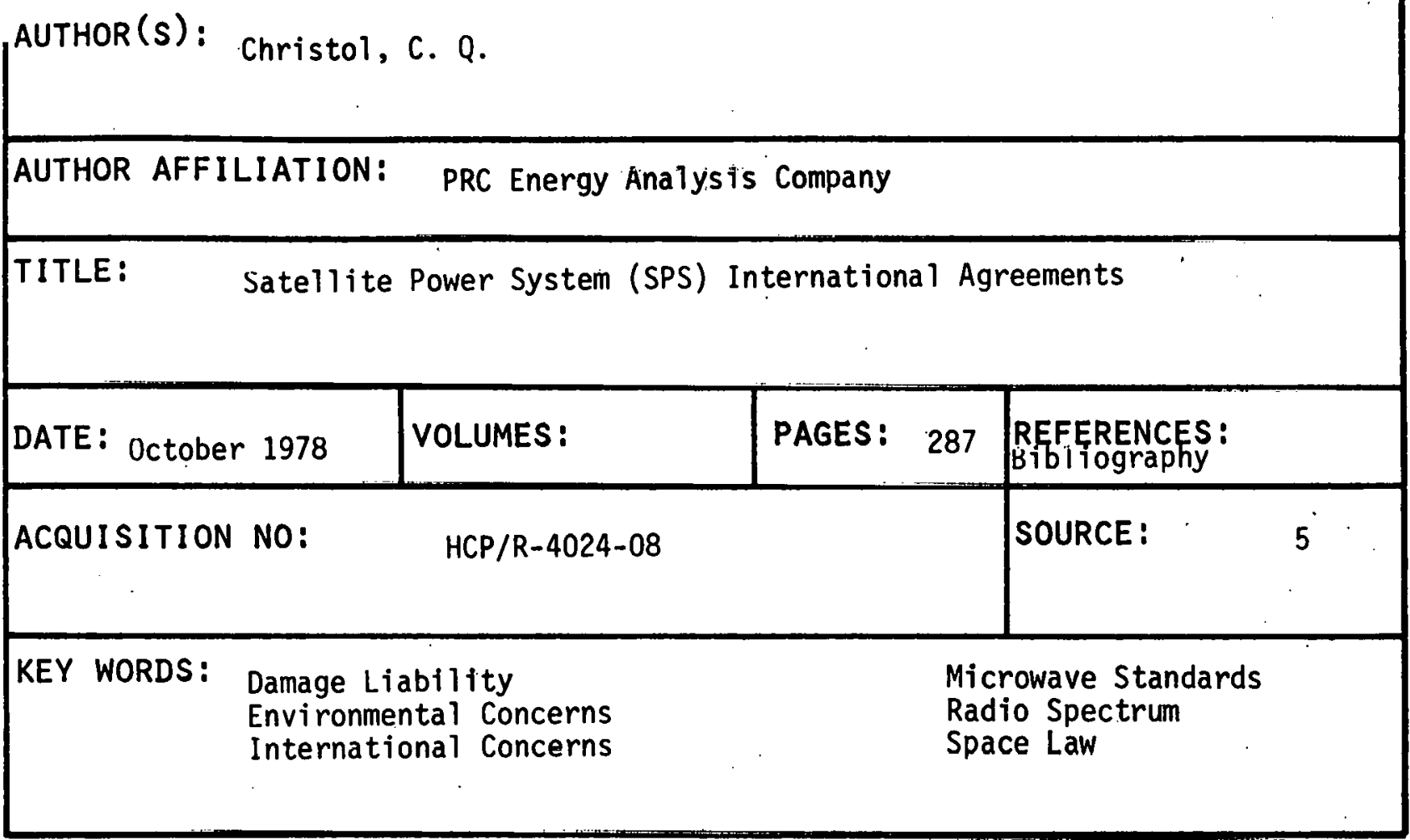

\begin{tabular}{|c|c|c|c|c|c|}
\hline SUBJECT: & \multicolumn{3}{|c|}{ SOCIAL ISSUES } & \multicolumn{2}{|c|}{ SPSPO INDEX NO: SI-5 } \\
\hline \multicolumn{6}{|c|}{ AUTHOR(s): Citizens' Energy Project } \\
\hline \multicolumn{6}{|c|}{ AUTHOR AFFILIATION: Citizens' Energy Project } \\
\hline TITLE: & \multicolumn{5}{|c|}{ Satellite Power System Program Summary Series } \\
\hline DATE: 1978 & & VOLUMES: & PAGES: 69 & REFERENCES : & None \\
\hline ACQUISITION & no: & By Title, 22 White & Paperś & SOURCE: & 31 \\
\hline KEY WORDS: & \multicolumn{3}{|c|}{$\begin{array}{l}\text { Environmental Concerns } \\
\text { International Concerns } \\
\text { Key Issues }\end{array}$} & \multicolumn{2}{|l|}{$\begin{array}{l}\text { Land Use } \\
\text { Military Issues } \\
\text { Public Outreach }\end{array}$} \\
\hline
\end{tabular}




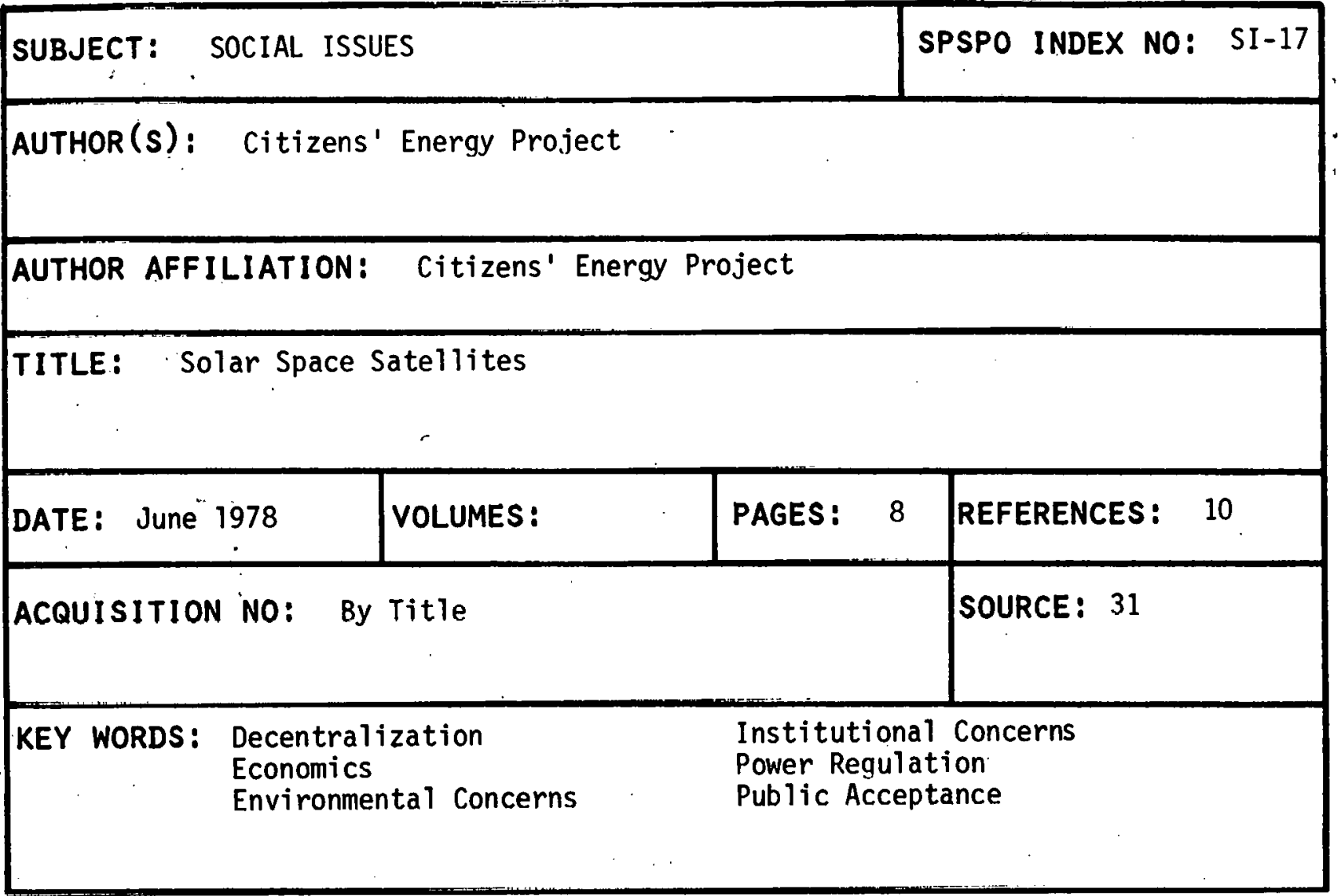

SUBJECT: SOCIAL ISSUES

$\begin{aligned} \operatorname{AUTHOR}(s): \begin{array}{l}\text { (1) } \\ \text { (2) Hill, M. }\end{array} & \text { (2) Rabe, J. A. } \\ \text { (3) Kotin, A. D. } & \end{aligned}$

\begin{tabular}{ll}
\hline AUTHOR AFFILIATION: & (1) None \\
& (2) Environmental Resources Group \\
3 & Kotin, Regan and Mouchly, Inc.
\end{tabular}

TITLE: Satellite Power System (SPS) Utility Integration: Institutional Planning and Operational Issues

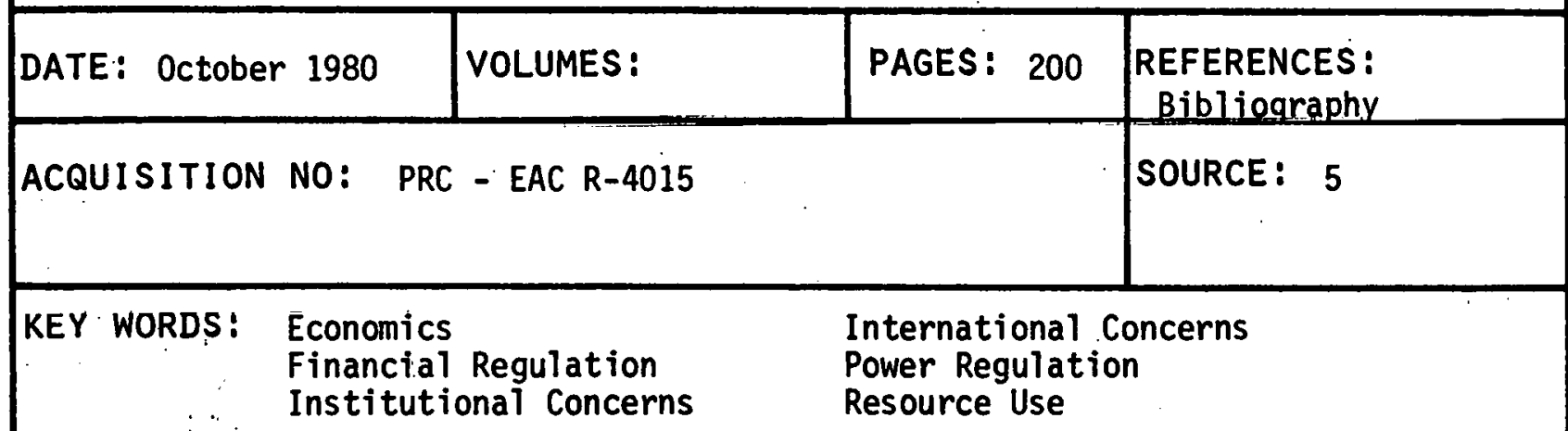


SUBJECT: SOCIAL ISSUES

UTHOR (S): (1) Criswell, D.R.

(4) O'Leary, B. T.

(2) Glaser, P. E.

(3) Mayur, R.

(4) $0^{\prime}$ Neill, G. K.

(5) Vajk, J. P.

AUTHOR AFFILIATION: (1) Lunar and Planetary Institute (2) Arthur D. Little, Inc. (3) Urban. Development Institute (India) (4) Princeton University

(5) Science Applications, Inc.

TITLE: The Role of Space Technology in the Developing Countries

DATE : 1980

VOLUMES:

PAGES: 11

REFERENCES : None

ACQUISITION NO:

0191-9067/80/010097-11

Space Solar Power Review, Vol. 1,

SOURCE: 33 pp. 97-107

KEY WORDS: Economics

Energy Options

International Concerns

SUBJECT: SOCIAL ISSUES

SPSPO INDEX NO: SI-33

AUTHOR(S): Daurio, A: P.

AUTHOR AFFILIATION: PRC Energy Analys is Company

TITLE: Determinants of a Strategy for International Participation in an SPS Program

DATE: December 1980

VOLUMES:

PAGES : 54

REFERENCES:

ACQUISITION NO: DOE/ER/10041-T13

SOURCE : 5

KEY WORDS: Economics

Energy Options

Financial Regulation
International Concerns

International Interest

Power Regulation 
AUTHOR(s): Duffy-Armstrong, M.

AUTHOR AFFILIATION: SRI International

TITLE: A Technology Assessment of Solar Energy Systems: Community Impediments to Implementation of Solar Energy

\begin{tabular}{|c|c|c|c|c|}
\hline DATE: Novemb & ber 1979 & VOLUMES: & PAGES : 119 & REFERENCES: 35 \\
\hline \multicolumn{3}{|c|}{ ACQUISITION NO: } & & SOURCE: 5 \\
\hline KEY WORDS: & \multicolumn{2}{|c|}{$\begin{array}{l}\text { Decentralization } \\
\text { Economics } \\
\text { Financial Regulation }\end{array}$} & \multicolumn{2}{|c|}{$\begin{array}{l}\text { Industry } \\
\text { Institutional Concerns } \\
\text { Terrestrial Solar }\end{array}$} \\
\hline
\end{tabular}

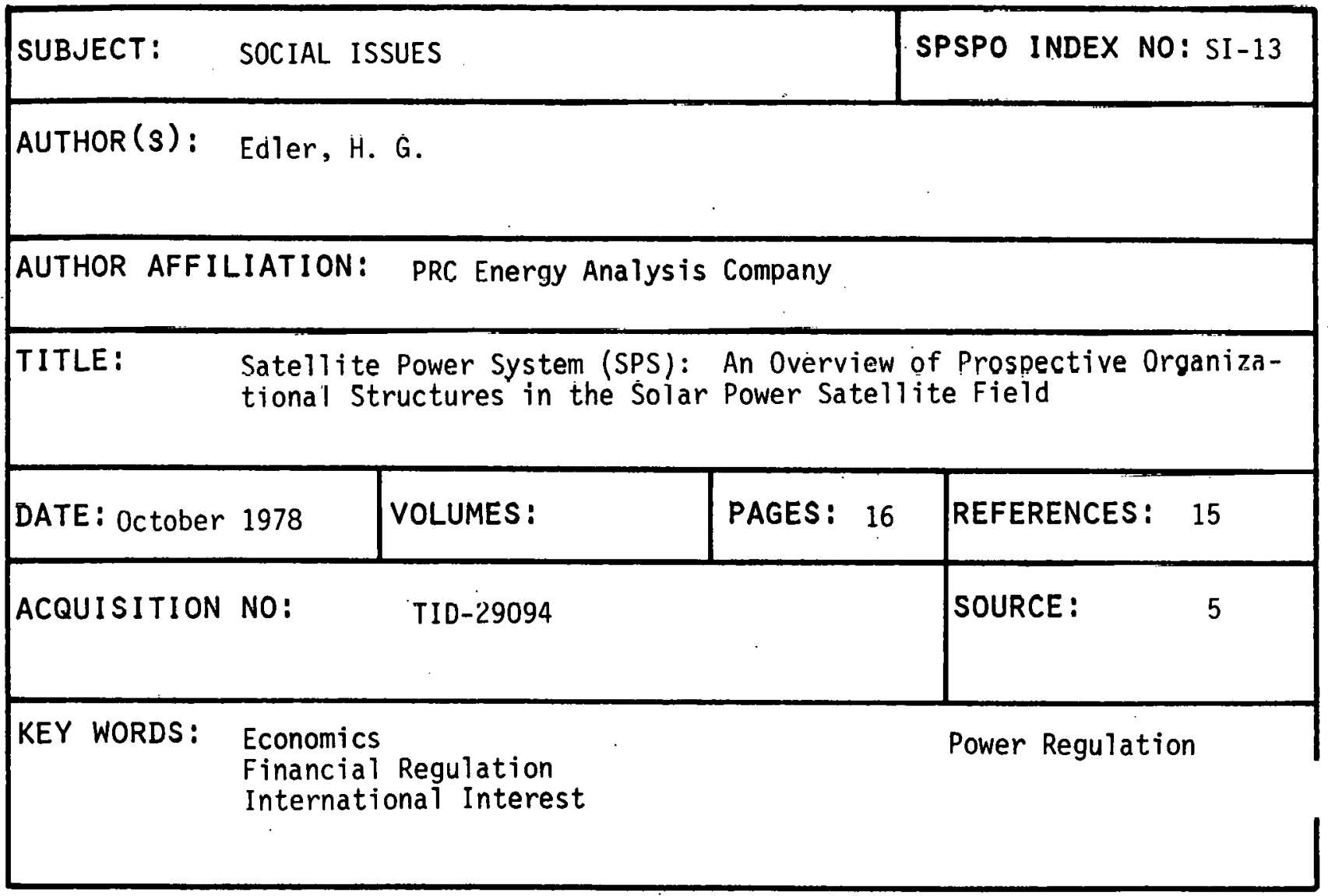


AUTHOR (S): Energy, U. S. Department of

AUTHOR AFFILIATION: U. S. Department of Energy

TITLE: Some Questions and Answers About the Satellite Power System (SPS)

\begin{tabular}{|c|c|c|c|}
\hline DATE: January 1980 & VOLUMES : & PAGES : 44 & REFERENCES: 67 \\
\hline \multicolumn{3}{|c|}{ ACQUISITION NO: DOE/ER-0049/1 } & SOURCE: 5 \\
\hline \multicolumn{2}{|c|}{$\begin{aligned} \text { KEY WORDS: } & \text { Damage Liability } \\
& \text { Economics } \\
& \text { Environmental Concerns }\end{aligned}$} & \multicolumn{2}{|c|}{$\begin{array}{l}\text { Institutional Concerns } \\
\text { International Concerns } \\
\text { Land Use. }\end{array}$} \\
\hline
\end{tabular}

\begin{tabular}{|l|l|}
\hline SUBJECT: SOCIAL ISSUES & SPSPO INDEX NO: SI-19 \\
\hline
\end{tabular}

AUTHOR(S): Forum for the Advancement of Students in Science and Technology, Inc.

AUTHOR AFFILIATION: Forum for the Advancement of students in Science and Technology, Inc.

TITLE: Satellite Power System (SPS) FASST Briefing

\begin{tabular}{|l|l|l|l|}
\hline DATE: JuTy 1979 & VOLUMES: & PAGES: 76 & REFERENCES : None \\
\hline ACQUISITION NO: By Title & SOURCE: 38 \\
\hline KEY WORDS: $\begin{array}{l}\text { Decentralization } \\
\begin{array}{l}\text { Environmental Concerns } \\
\text { Institutional Concerns }\end{array}\end{array}$ & $\begin{array}{l}\text { International Concerns } \\
\text { Military Issues } \\
\text { Student Interest } \\
\end{array}$ & \\
\hline
\end{tabular}


SUBJECT： SOCIAL ISSUES

AUTHOR(s): George Washington University

AUTHOR AFFILIATION: George Washington University

TITLE: Social Assessment of On-Site Solar Energy

\begin{tabular}{|l|l|l|l|}
\hline DATE: April 1979 & VOLUMES: & PAGES: 235 & REFERENCES: 197 \\
\hline
\end{tabular}

ACQUIBITION NO: $\quad$ HCP/R4040-02

SOURCE: 5

KEY WORDS: Decentralization

Economics

Energy Options

Public. Acceptance

Resource Use

Terrestrial Solar

SUBJECT: SOCIAL ISSUES

AUTHOR(s): George Washington University

AUTHOR AFFILIATION: George Washington University

TITLE : Energy Implications of an Aging Population

\begin{tabular}{|l|l|l|l|}
\hline DATE: August 1980 & VOLUMES: & PAGES: 64 & REFERENCES: 17 \\
\hline ACQUISITION NO: DOE/ER/10041-01 & SOURCE: 5 \\
\hline
\end{tabular}

KEY WORDS: Demographics

Economics

Population 
SUBJECT: SOCIAL ISSUES

AUTHOR(s): Gerlach, L.P.

AUTHOR AFFILIATION: University of Minnesota

TITLE: Technological Innovation and Social Exploration in Economic Growth . and Energy Development

\begin{tabular}{|c|c|c|c|}
\hline DATE: May 1977 & VOLUMES: & PAGES: 12 & REFERENCES : 42 \\
\hline \multicolumn{2}{|c|}{ ACQUISITION NO: $\quad 77-558$} & & SOURCE: 2 \\
\hline KEY WORDS : & $\begin{array}{l}\text { Decentralization } \\
\text { Economics } \\
\text { Energy Options }\end{array}$ & \multicolumn{2}{|c|}{$\begin{array}{l}\text { Environmental Concerns } \\
\text { Public Acceptance } \\
\text { Resource Use }\end{array}$} \\
\hline
\end{tabular}

\section{SUBJECT: $\quad$ SOCIAL. ISSUES}

AUTHOR(S): Gorove, S.

AUTHOR AFFILIATION: PRC Energy Analysis Company

TITLE: $\quad$ Satellite Power System (SPS) International Agreements

\begin{tabular}{|l|l|l|l|}
\hline DATE: October 1978 & VOLUMES: 1 & PAGES: 80 & $\begin{array}{l}\text { REFERENCES: } \\
\text { Bibliography }\end{array}$ \\
\hline ACQUISITION NO: & HCP/R-4024-12 0 & SOURCE: \\
\hline
\end{tabular}

KEY WORDS: International Concerns

Key Issues

Radio Spectrum

Space Law 
SUBJECT: SOCIAL ISSUES

SPSPO INDEX NO: SI-12

$\operatorname{AUTHOR}(S)$ : Hazelrigg, G. A.

AUTHOR AFFILIATION:

ECON Incorporated

TITLE: Polftical and Legal Implications of Developing and Operating a Satellite Power System

\begin{tabular}{|l|l|l|l|}
\hline DATE: August 1977 & VOLUMES: & PAGES: 244 & $\begin{array}{l}\text { REFERENCES: } \\
\text { Bibliography }\end{array}$ \\
\hline ACQUISITION NO: $\quad \begin{array}{l}77-195-1 \\
\text { JPL } 954652\end{array}$ & SOURCE: \\
\hline KEY WORDS: & $\begin{array}{l}\text { Economics } \\
\text { International Concerns } \\
\text { Military Issues }\end{array}$ & $\begin{array}{l}\text { Power Regulation } \\
\text { Satellite Vulnerability } \\
\text { Space Law }\end{array}$
\end{tabular}

SUBJECT: SOCIAL ISSUES

SPSPO INDEX NO: SI-25

AUTHOR(S): Klineberg, S. I.

AUTHOR AFFILIATION: Rice University

TITLE: Social Acceptability of Satellite Power Systems (SPS): The Near Term Outlook

\begin{tabular}{|l|l|l|l|}
\hline DATE: May 1980 & VoluMES: & PAGES: 58 & $\begin{array}{l}\text { REFERENCES: } \\
\text { Btbliography }\end{array}$ \\
\hline ACQUISITION NO: By Title & SOURCE: 13 \\
\hline KEY WORDS: $\begin{array}{l}\text { Decentralization } \\
\text { Power Regulation } \\
\text { Public Acceptance }\end{array}$ & \\
\hline
\end{tabular}


SUBJECT: SOCIAL ISSUES

SPSPO INDEX NO: SI-8

UTHOR (S): Kotin, A. D.

AUTHOR AFFILIATION: Allan D. Kotin Economic Consultants

TITLE: Satellite Power System (SPS) State and Local Regulations as Applied to Satellite Power System Microwave Receiving Antenna Facilities

DATE: October 1978 \begin{tabular}{|l|l|ll}
\hline VOLUMES: & PAGES: 90 & REFERENCES: 164 \\
\hline
\end{tabular}

ACQUISITION NO:

HCP/R-4024-05

SOURCE :

5

KEY WORDS: Economics

Financial Regulation

International Concerns

Land Use

Power Regulation

SUBJECT: $\quad$ SOCIAL ISSUES

SPSPO INDEX NO: SI-Y

AUTHOR'(s): Ladwig, A.

David, L.

AUTHOR AFFILIATION: Forum for the Advancement of Students in Science and Technology (FASST)

TITLE: Satelitite Power System (SPS) Student Participation

\begin{tabular}{|l|l|l|lc|}
\hline DATE: 0 October 1978 & VOLUMES: & PAGES: 91 & REFERENCES: 148 \\
\hline ACQUISITION NO: $\quad$ HCP/R-4024-06 & SOURCE: & 5 \\
\hline
\end{tabular}

KEY WORDS: Public Outreach

Student Interest 
AUTHOR(s): Mc Neal, S. R.

AUTHOR AFFILIATION: PRC Energy Analys is Company

TITLE: Satellite Power System (SPS) Public Outreach Experiment

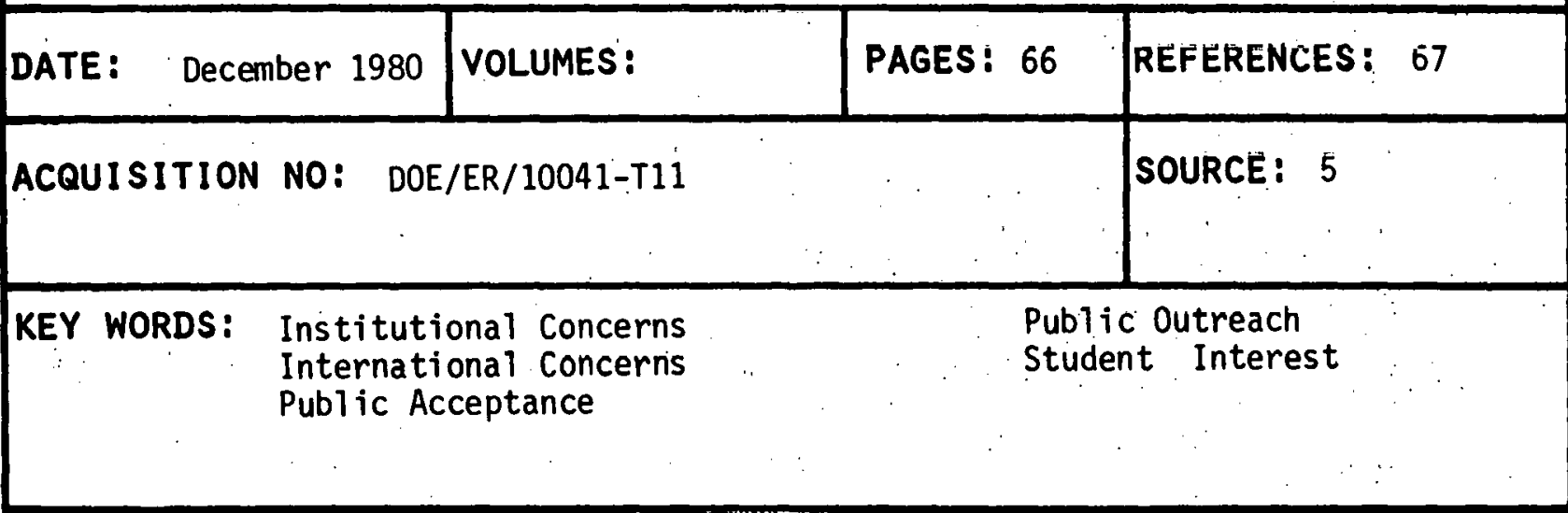

SUBJECT: SOCIAL ISSUES

SPSPO INDEX NO: SI-28

AUTHOR(S): Marsh and McLellen, Inc.

AUTHOR AFFILIATION: Marsh and McLellen, Inc.

TITLE: Sateilite Power System (SPS) Initial Insurance Evaluation

\begin{tabular}{|c|c|c|c|}
\hline DATE: September 1980 & VOLUMES: & PAGES: 31 & REFERENCES: 11 \\
\hline \multicolumn{3}{|c|}{ ACQUISITION NO: DOE/ER/21059-01 } & SOURCE: 5 \\
\hline
\end{tabular}

Institutional Concerns 


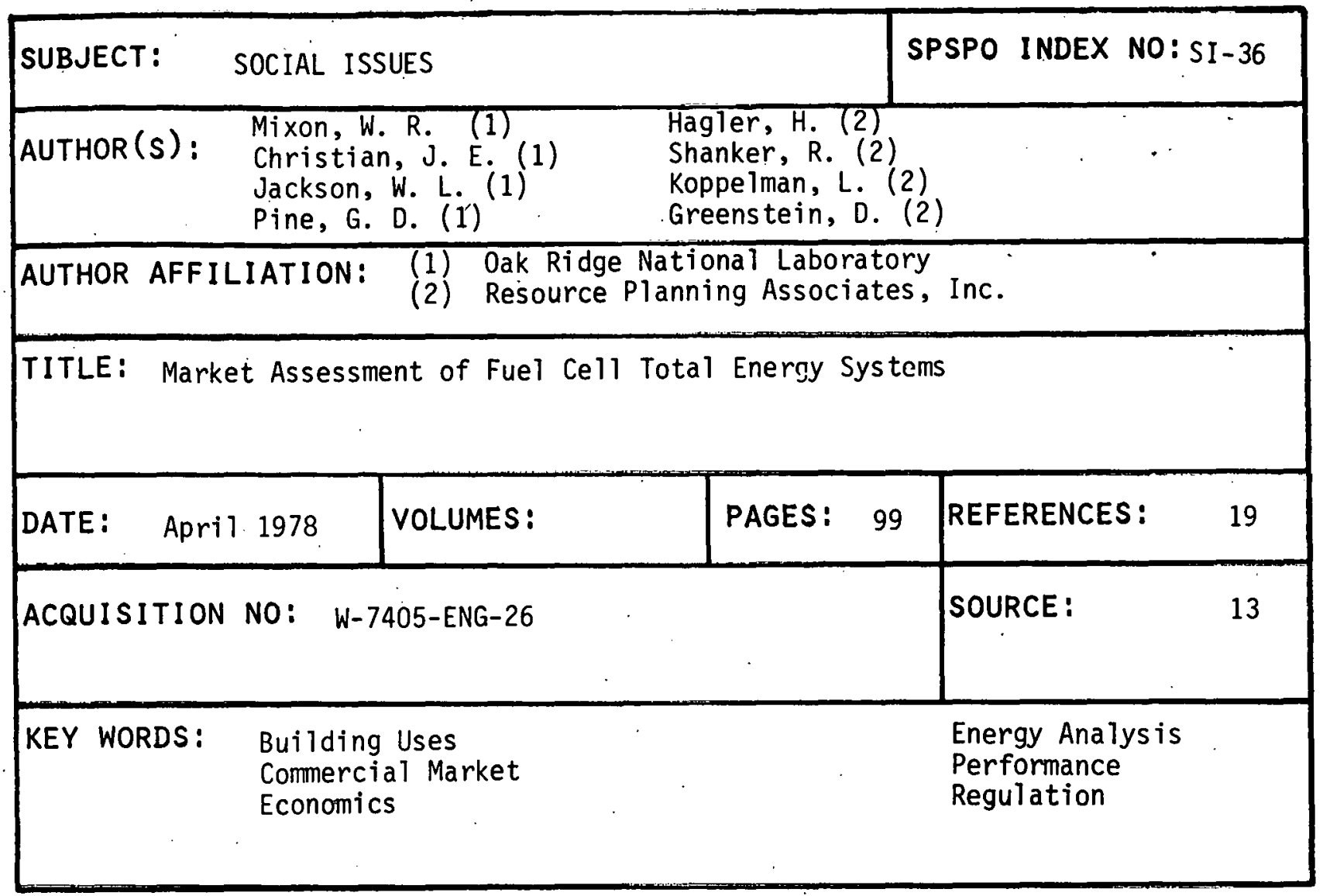

\section{SUBJECT: SOCIAL ISSUES}

$\operatorname{AUTHOR}(s)$ : Naisbitt, J.

AUTHOR AFFILIATION: PRC Energy Analysis Company

TITLE: Satellite Power System (SPS) Centralization/Decentralization

\begin{tabular}{|c|c|c|c|c|c|}
\hline DATE : october & 1978 & VOLUMES: & PAGES: 63 & \multicolumn{2}{|c|}{$\begin{array}{l}\text { REFERENCES: } \\
\text { Bibfiography }\end{array}$} \\
\hline ACQUISITION & NO: & \multicolumn{2}{|c|}{$H C P / R-4024-09$} & SOURCE: & 5 \\
\hline KEY WORDS: & $\begin{array}{l}\text { Econon } \\
\text { Energy } \\
\text { Power }\end{array}$ & $\begin{array}{l}\text { ions } \\
\text { dlation }\end{array}$ & \multicolumn{3}{|c|}{ Public Acceptance } \\
\hline
\end{tabular}


SUBJECT: SOCIAL ISSUES

AUTHOR(s): National Space Institute

AUTHOR AFFILIATION: National Space Institute

TITLE: Solar Power Satellite Issue Guides

\begin{tabular}{|c|c|c|c|c|}
\hline \multicolumn{2}{|c|}{ DATE: October 1978} & VOLUMES: & PAGES: 40 & $\begin{array}{l}\text { REFERENCES : } \\
\text { Bibliography }\end{array}$ \\
\hline \multicolumn{4}{|c|}{ ACQUISITION NO: By Title } & SOURCE: 38 \\
\hline KEY WORDS: & \multicolumn{2}{|c|}{$\begin{array}{l}\text { Environmentäl Concerns } \\
\text { Institutional Concerns } \\
\text { International Concerns }\end{array}$} & \multicolumn{2}{|c|}{$\begin{array}{l}\text { Military Issues } \\
\text { Satellite Vulnerability }\end{array}$} \\
\hline
\end{tabular}

\begin{tabular}{|l|l|}
\hline SUBJECT: SOCIAL ISSUES & SPSPO INDEX NO: SI-3 \\
\hline AUTHOR (S): ozeroff, M. J. &
\end{tabular}

AUTHOR AFFILIATION: PRC Energy Analys is Corporation

TITLE: Satellite Power System (SPS) Military Implications

\begin{tabular}{|l|l|l|l|}
\hline DATE: OCtober 1978 & VOLUMES: & PAGES: 43 & $\begin{array}{l}\text { REFERENCES: } \\
\text { Bibliography }\end{array}$ \\
\hline ACQUISITION NO: $\quad$ HCP/R-4024-01 & SOURCE: \\
\hline
\end{tabular}

KEY WORDS: International Concerns

Military Issues

Satellite Vulnerability 


$$
\begin{aligned}
\operatorname{UTHOR}(s): & \text { Poch, L. A. } \\
& \text { Wolsko, T. D. }
\end{aligned}
$$

AUTHOR AFFILIATION: Argonne National Laboratory

\begin{tabular}{|c|c|c|c|}
\hline DATE: October 1979 & VOLUMES: & PAGES: 19 & REFERENCES : 13 \\
\hline ACQUISITION NO: & EES-TM-67 & & SOURCE : 5 \\
\hline
\end{tabular}

TITLE: A Review of the Nuclear Safeguards Problem

KEY WORDS: Institutional Concerns

International Concerns

Public Acceptance

SUBJECT: SOCIAL ISSUES

SPSPO INDEX NO: SI-31

$\operatorname{AUTHOR}(s)$ : PRC Energy Analys is Company

AUTHOR AFFILIATION: PRC Energy Analys is Company

\begin{tabular}{|c|c|c|}
\hline DATE: & December 1980 & VOLUMES: \\
\hline
\end{tabular}

TITLE: Satellite Power System (SPS) Societal Assessment

ACQUISITION NO: DOE/ER/10041-T12

KEY WORDS: Economics

Energy Options

Environmental Concerns \begin{tabular}{l|l} 
PAGES: 55 & REFERENCES: 31
\end{tabular}
SOURCE: 5

Institutional Concerns International Concerns Public Outreach 
$\operatorname{AUTHOR}(S)$ : Ritschard, R. L.

AUTHOR AFFILIATION: Lawrence Berkeley Laboratory

TITLE : Assessment of Solar Energy Within a Community: Summary of Three Community-Level Studies

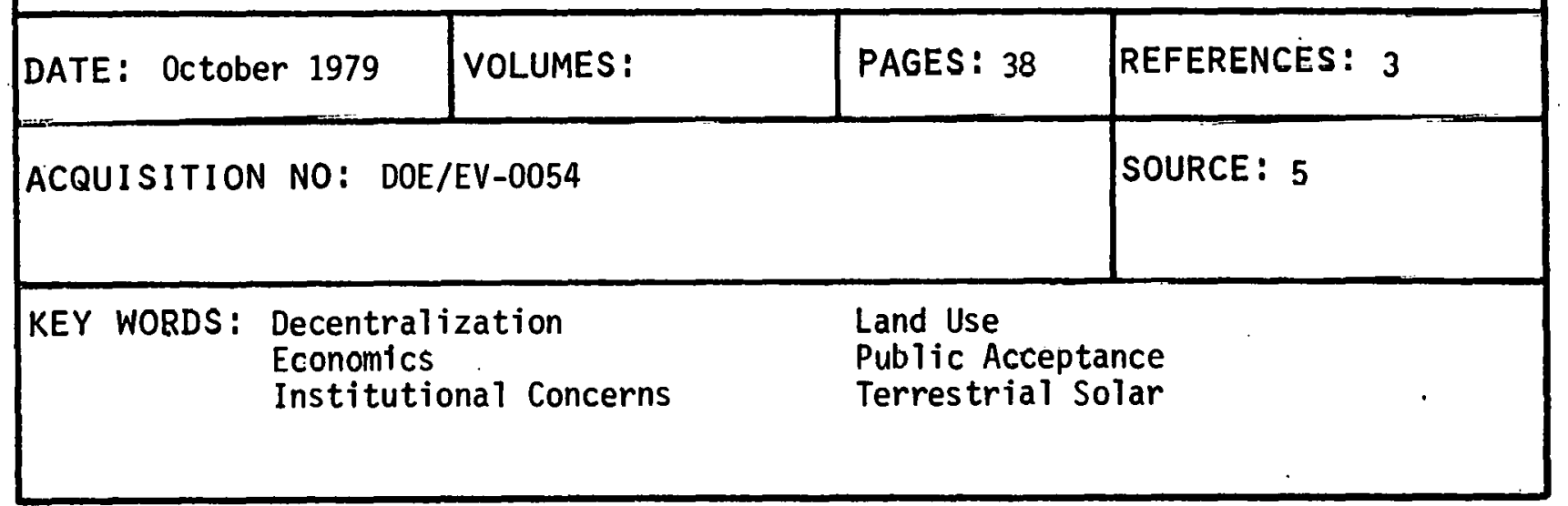

SUBJECT: SOCTAL ISSUES

AUTHOR(S): Ruth, J.

Westpha $1, W$.

AUTHOR AFFILIATION: European Space Agency

TITLE:

Study on European Aspects of Solar Power Satellites

\begin{tabular}{|l|l|l|l|}
\hline DATE: June 1979 & VOLIIMES: & PAGES: 131 & $\begin{array}{l}\text { REFERENCES: } \\
\text { Bibliography }\end{array}$ \\
\hline ACQUISITION NO: & $3705 / 78 / \mathrm{F} / \mathrm{DK}(\mathrm{SC})$ & SOURCE: \\
\hline
\end{tabular}

KEY WORDS: International Interest

Western Europe 


\section{SPACE TRANSPORTATION \\ (CATEGORY ST)}

Reports in this category deal only with space transportation technology, advancements and concepts and are applicable to space ventures in general.

KEY WORDS:

Cargo Vehicle

Chemical Rocket

Cost

Economics

Electric Vehicle

Energy Requirements

Engineering Constraints

Heavy Lift Launch Vehicle

Laser Rocket

Nuclear Electric

Nuclear Rocket

Orbit Transport

Payload

Personnel Launch Vehicle

Propellant

Space Shuttle

Solar Electric

Thermal Systems

Thruster Concept
PAGE NUMBER :

$217,219,222$

218,221

$217,218,219,220,222$

218,222

217,222

220

217,221

217,222

$219,220,221$

218

218

$217,219,222$

$218,219,220,221$

217,222

220,221

218,221

218,221

220,221

$218,219,220,221$

RELATED CATEGORIES:

Atmospheric Effects (AE)

Economics and Finances (EF)

Resources and Materials (RM)

Systems Analyses (SA)

Space Utilization (SU) 
THIS PAGE

\section{WAS INTENTIONALLY}

\section{LEFT BLANK}


$\operatorname{AUTHOR}(s)$ : Alabama, University of

AUTHOR AFFILIATION: University of Alabama

TITLE: The Final Report of the SPS Space Transportation Workshop

DATE: October 1980 VOLUMES:

PAGES: 140 REFERENCES: 9

ACQUISITION NO: By Title, NAS 8 - 33132

SOURCE : 4

KEY WORDS: Cargo Vehicle Electric Vehicle

Heavy Lift Launch Vehicle

Oransfer

Engineering Constraints Personnel Launch Vehicle

SUBJECT: SPACE TRANSPORTATION

SPSPO INDEX NO: ST-7

AUTHOR(s): Battelle Columbus Laboratories

AUTHOR AFFILIATION: Battelle Columbus Laboratories

TITLE: Satellite Power Systems (SPS) Space Transportation Cost Analysis and Evaluation

DATE: November 1980

VOLUMES:

PAGES: 138

REFERENCES : 10

ACQUISITION NO: DOE/ER-0086

SOURCE : 5

KEY WORDS: Cargo Vehicle

Cost

Electric Vehicle
Heavy Lift Launch Vehicle

Orbit Transport

Personnel Launch Vehicle 


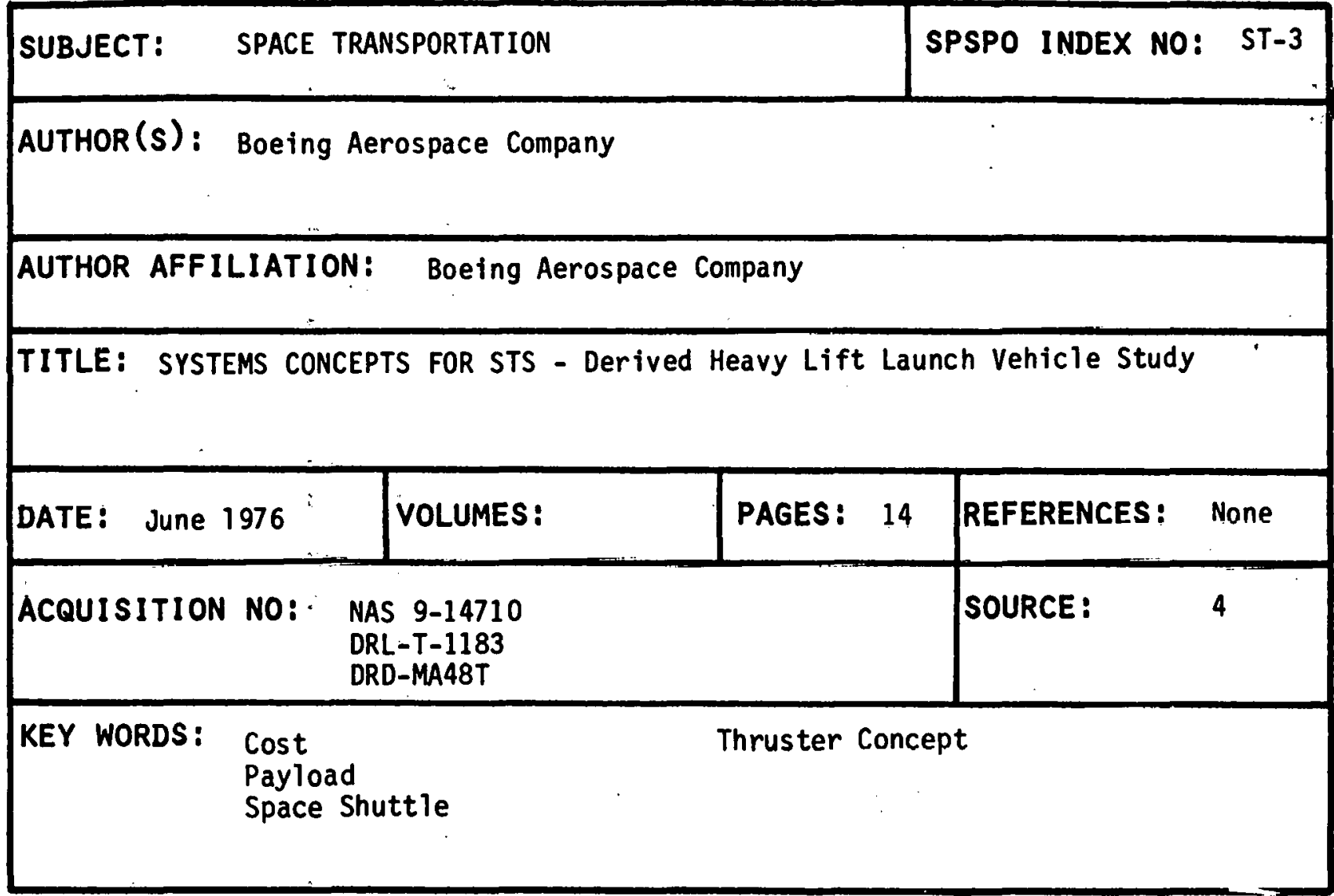

\begin{tabular}{|c|c|c|c|c|c|}
\hline SUBJECT: & \multicolumn{2}{|c|}{ SPACE TRANSPORTATION } & & \multicolumn{2}{|c|}{ SPSPO INDEX NO: ST-2 } \\
\hline $\operatorname{AUTHOR}(s):$ & \multicolumn{5}{|c|}{ Helms, I. L. (Editor) } \\
\hline \multicolumn{6}{|c|}{ AUTHOR AFFILIATION: National Aeronautics and Space Administration } \\
\hline TITLE : & \multicolumn{5}{|c|}{ Advanced Propulsion Comparison Study } \\
\hline DATE: April & 1973 & VOLUMES: & PAGES: 205 & REFERENCES : & 53 \\
\hline ACQUISITION & \multicolumn{3}{|c|}{ NO: By Title } & SOURCE: & 4 \\
\hline KEY WORDS : & \multicolumn{4}{|c|}{$\begin{array}{l}\text { Chemical Rocket } \\
\text { Economics } \\
\text { Nuclear Electric }\end{array}$} & \\
\hline
\end{tabular}


IUTHOR (s):

(1) Hertzberg, A.

(1) Sun, $K$.

(2) Jones, W. S.

AUTHOR AFFILIATION: (1) University of Washington

(2) Lockheed Missiles and Space Company

TITLE: A Laser Powered Flight Transportation System

\begin{tabular}{|l|l|l|l|}
\hline DATE: August 1978 & VOLUMES: & PAGES: 30 & REFERENCES: 28 \\
\hline ACQUISITION NO: 78-1484 & SOURCE: 2 \\
\hline KEY WORDS: $\begin{array}{ll}\text { Cost } \\
\text { Laser Rocket } \\
\text { Orbit Transport }\end{array}$ & $\begin{array}{l}\text { Payload } \\
\text { Thruster Concept }\end{array}$ \\
\hline
\end{tabular}

\section{SUBJECT: SPACE TRANSPORTATION}

AUTHOR (S): Kramer, P. R.

Buhler, R. D.

AUTHOR AFFILIATION: Universitat Stuttgart (Germany)

TITLE: Hybrid Rocket/Air-Breathing Propulsion for Ballistic Space Transportation - Project ITUSTRA

DATE: Apri.1 1979 VOLUMES:

PAGES: 10

REFERENCES:

ACQUISITION NO: $79-7038$

SOURCE： 2

KEY WORDS: Cargo Vehicle

Payload

Thruster Concept 
SUBJECT: SPACE TRANSPORTATION

SPSPO INDEX NO: ST-4

AUTHOR(s): Lockheed Missiles and Space Company, Inc.

AUTHOR AFFILIATION: Lockheed Missiles and Space Company, Inc.

TITLE: Laser Rocket System Analysis Study

\begin{tabular}{|l|l|l|l|}
\hline DATE: August 19\%8 & VOLUMES: & PAGES: 190 & REFERENCES: 20 \\
\hline ACQUISITION NO: LMSC-D564671A & SOURCE: 4 \\
\hline
\end{tabular}

KEY WORDS: Cost Thruster Concept

Laser Rocket

Propellant

SUBJECT: SPACE TRANSPORTATION

SPSPO INDEX NO: ST-9

$\operatorname{AUTHOR}(S)$ : Rice, E. E.

AUTHOR AFFILIATION: Battelle Columbus Laboratories

TITLE: Energy Impact Assessment of NASA's Past, Present and Future Space Launch Vehicles

\begin{tabular}{|l|l|l|l|}
\hline DATE: May-June 1978 & VolUMES: & PAGES: 7 & REFERENCES: 20 \\
\hline ACQUISITION NO: By Title; Journal of Energy, & $\begin{array}{l}\text { Vol.2, No. 3, pp 182-188 } \\
\text { VOURCE: }\end{array}$ & \\
\hline
\end{tabular}

KEY WORDS: Energy Requirements

Payload

Technology 
THOR(S): Weiss, R. F.

Pirri, A.N.

Kemp, N. H.

AUTHOR AFFILIATION: Physical Sciences, Inc.

TITLE: Laser Propulsion

DATE: March 1979

\section{VOLUMES:}

PAGES: 9

REFERENCES : $\quad 30$

ACQUI Astronautics and Aeronautics

Vol. 17, No. 3

SOURCE :

pp. 50-58

KEY WORDS :

Engineering Constraints

Laser Rocket

Thermal Systems

Propellant

SUBJECT: SPACE TRANSPORTATION

SPSPO INDEX NO: ST-5

AUTHOR (s): Woodcoek, G. R.

AUTHOR AFFILIATION: Boeing Aerospace Company

TITLE: Solar Power Satellite System Definition Study, Space Transportation Analys is

DATE: June 1980

VOLUMES:

PAGES: 150

REFERENCES : 9

ACQUISITION NO: D180-25969-5

NAS $9-15636$

\section{SOURCE :}

KEY WORDS: Chemical Rocket

Economics

Payload

Propellant

Space Shuttle

Solar Electric 
AUTHOR(s): Wolfe, M. G.

AUTHOR AFFILIATION: Aerospace Corporation

TITLE: Satellite Power System (SPS) Transportation Cost Assessment Study

DATE: January 1981

\begin{tabular}{ll|l}
\hline VOLUMES: & $\cdots$ & PAGES:
\end{tabular}

87

REFERENCES : $\quad 45$

ACQUISITION NO: ATR-81 (7898)-1

NAS Contract PRM-7919687

SOIIRCE : 27

KEY WORDS: Cargo Vehicle Cost

Electric Vehicle

Heavy Lift Launch Vechicle Orbit Transport

Personnel Launch Vehicle 


\section{SPACE UTILIZATION \\ (CATEGORY SU)}

The space utilization category includes reports and papers concerning a wide range of issues and interests about exploring and using space (including other planets) to fulfill and expand upon man's social needs and goals.

KEY WORDS :

Bogota. Declaration

Communications

Computer Model

Economics

Energy Demands

Energy Source

Future Projections

Habitat Requirements

Industrialization

Interference Effects

International Cooperation

Lunar Mining

Manned Flight

Manufacturing

Material Processes

Material Resources

National Programs

Neutrinn

Outer Space Treaty

Particles

Process Designs

Resource Depletion

Satellite Uses

Slot Availability

Space Colonization

Space Experiments

Space Law

Space Properties
PAGE NUMBER :

228,235

$225,228,230$

236,237

$229,232,233,234,235,237$

237

$229,230,231,234,235,236$

225

$225,229,231,232,237$

$225,226,227,229,231,233,234,235$

232

$227,228,230,232,235$

226,229

$225,229,230,231,234$

$226,227,231,233,234,235$

226,233

$225,226,227,235$

$230,232,233$

230

$227,228,235$

230

226

237

$225,227,228,230,233,234,236$

$225,227,228,232$,

$225,229,231,232,233,234,235,236$, 237

230

$227,228,235$

$225,227,232,233$ 
(Space Utilization

Category SU continued)

KEY WORDS:

Space Shuttle

Space Transmission

Spectrum Usage.

Technology

Theory

Unmanned Exploration
PAGE NUMBER:

233

229,232

$227,228,232$

220

$230,233,234,235,236$

$230,233,234$

RELATED CATEGORIES:

Biologic Effects (BE)

Environmental Effects (EE)

Economics and Finances (EF)

Resources and Materials (RM)

Systems Analyses (SA)

Systems Engineering (SE)

Space Transportation (ST) 


\section{SUBJECT: SPACE UTILIZATION}

AUTHOR(s): Abdulezer, L.

AUTHOR AFFILIATION: Polytechnic Institute of New York

TITLE: Consideration in the Design of Large-Scale Space Habitats

\begin{tabular}{|l|l|l|}
\hline DATE: $\quad$ November 1977 & VOLUMES: \\
\hline
\end{tabular}

ACQUISITION NO: The Polytechnic Engineer

Vol. XVIII, No. 1

pp. $28-41,53,56$

PAGES: 16 REFERENCES: 16

SOURCE :

KEY WORDS: Habitat Requi rements

Material Resources

Slot Availability

Space Colonization

Space Properties

SUBJECT: SPACE UTILIZATION

SPSPO INDEX NO: SU-26

AUTHOR(s): American Institute of Aeronautics and Astronautics

AUTHOR AFFILIATION: American Institute of Aeronautics and Astronautics

TITLE: Projected Space Technologies, Missions and Capábilities in the 2000-2020 Time Period

\begin{tabular}{|c|c|c|c|c|}
\hline DATE: Januar: & "y 1981 & VOLUMES: & PAGES: 208 & REFERENCES: 85 \\
\hline \multicolumn{4}{|c|}{ ACQUISITION NO: By Title } & SOURCE: 2 \\
\hline KEY WORDS : & $\begin{array}{l}\text { Communi } \\
\text { Future } \\
\text { Industr }\end{array}$ & $\begin{array}{l}\text { tions } \\
\text { jections } \\
\text { lization }\end{array}$ & $\begin{array}{l}\text { Manned Flight } \\
\text { Material Reso } \\
\text { Satellite Use }\end{array}$ & Irces \\
\hline
\end{tabular}




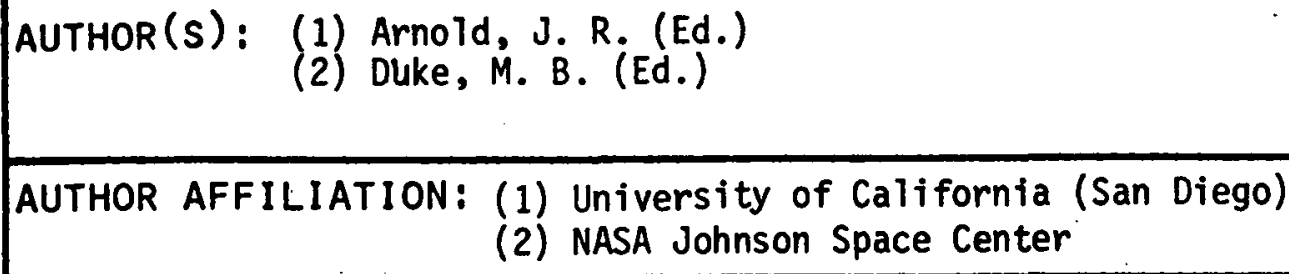

TITLE: Summer Workshop on Near-Earth Resources

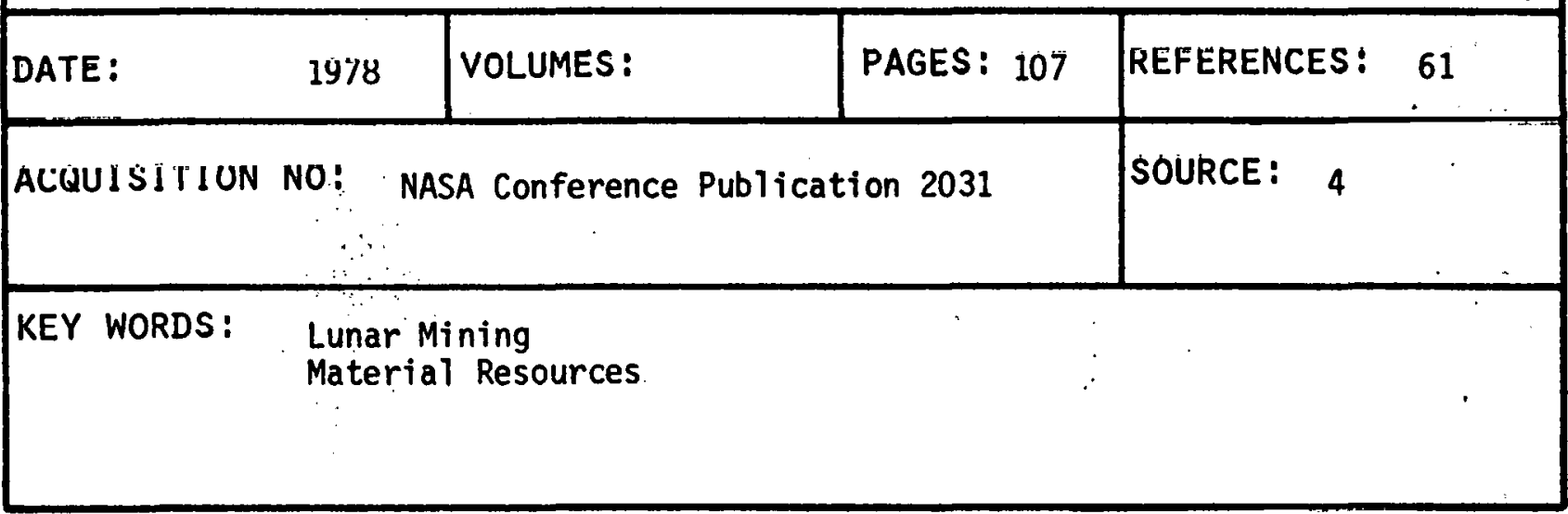

SUBJECT:

SPACE UTILIZATION

AUTHOR(S): Criswell, D. R.

AUTHOR AFFILIATION: Lunar and Planetary Institute

TITLE:

Extraterrestrial Materials Processing and Construction

DATE: September 1978

VOLUMES:

PAGES : 474

REFERENCES: $10 ?$

ACQUISITION NO: By Title

SOURCE:

18

KEY WORDS: Industrialization

Lunar Mining

Manufacturing

Material Processes

Material Resources

Process Designs 
AUTHOR(S): Freedman, R. S.

AUTHOR AFFILIATION: Polytechnic Institute of New York

TITLE: Techniques of Optimization Applied to Space Industrialization:

An Introduction

\begin{tabular}{|l|l|ll|}
\hline DATE: November 1977 & VOLUMES: & PAGES: 5 & REFERENCES: 7 \\
\hline ACQUISITION NO: $\begin{array}{l}\text { The Polytechnic Engineer } \\
\text { Vol. XVIII, No. 1 } \\
\text { pp. 44-47,56 }\end{array}$ & SOURCE: 11 \\
\hline KEY WORDS: & $\begin{array}{l}\text { Industrialization } \\
\text { Manufacturing } \\
\text { Material Resources }\end{array}$ & Space Properties \\
\hline
\end{tabular}

SUBJECT: SPACE UTILIZATION

AUTHOR(S): Galloway, E.

AUTHOR AFFILIATION: International Institute of Space Law

TITLE: The Future of Space Law

\begin{tabular}{|l|l|l|l|}
\hline DATE: OCtober 1976 & VOLUMES: & PAGES: 11 & REFERENCES: 35 \\
\hline ACQUISITION NO: IAF-ISL-76-06; 27th International & SOURCE: 42 \\
Astronautical Congress (Aliaheim) & & $\begin{array}{l}\text { Slot Availability } \\
\text { Space Law } \\
\text { Spectrum Usage }\end{array}$ \\
\hline KEY WORDS: & $\begin{array}{l}\text { International Cooperation } \\
\text { Outer Space Treaty } \\
\text { Satellite Uses }\end{array}$
\end{tabular}


AUTHOR(s): Gehrig, J. J.

AUTHOR AFFILIATION: U. S. Senate Staff

TITLE: Geostationary Orbit - Technology and Law

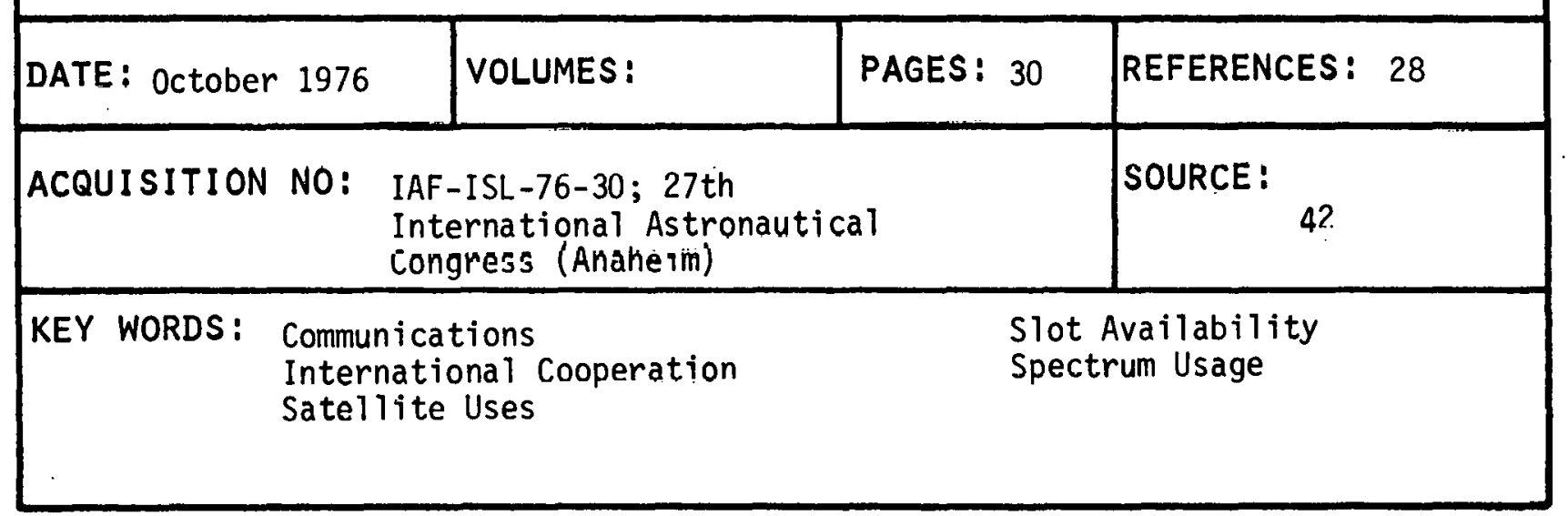

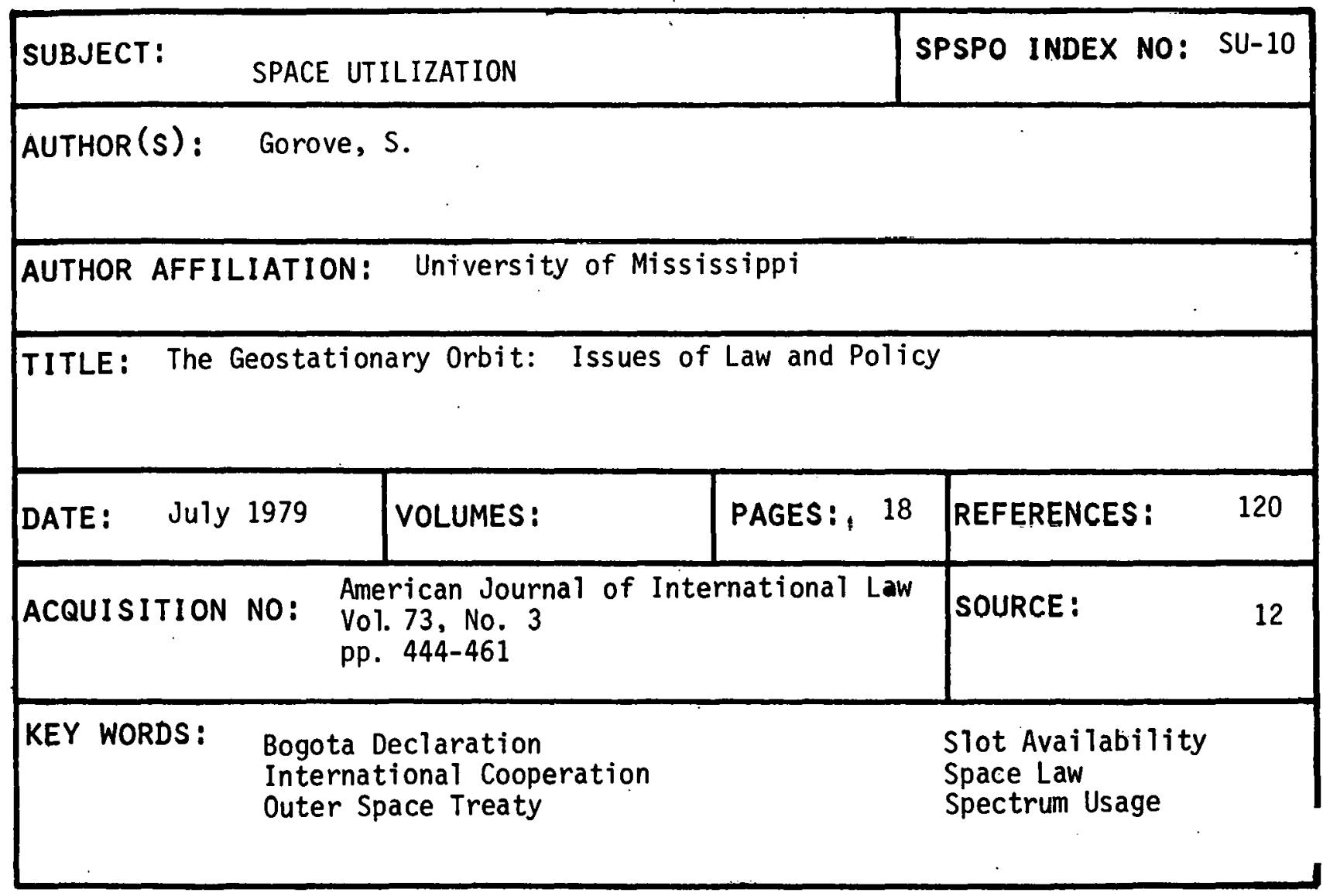


IUTHOR(s): Grey, J. (Ed.)

AUTHOR AFFILIATION: American Institute of Aeronautics and Astronautics

TITLE: Space Manufacturing Facilities 2 - Space Colonies

\begin{tabular}{|c|c|c|c|}
\hline DATE: September 197 & VOLUMES : & PAGES: 367 & REFERENCES : 586 \\
\hline ACQUISITION NO: $\frac{B}{P}$ & \multicolumn{2}{|c|}{$\begin{array}{l}\text { By Title (Proceedings of the Third } \\
\text { Princeton/AIAA Conference) }\end{array}$} & SOURCE : \\
\hline
\end{tabular}

KEY WORDS: Energy Source Industrialization Lunar Mining

Manned Flight

Space Colonization

Space Transmission

SUBJECT: SPACE UTILIZATION

SPSPO INDEX NO: SU-20

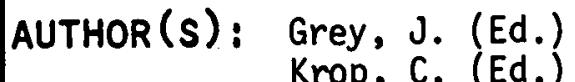

AUTHOR AFFILIATION: American Institute of Aeronautics and Astronautics

TITLE: Space Manufacturing III

\begin{tabular}{|l|l|l|l|}
\hline DATE: October 1979 & VOLUMES: & PAGES: 585 & REFERENCES: 959 \\
\hline ACQUISITION NO: $\begin{array}{l}\text { By Title; Proceedings of the } \\
\begin{array}{l}\text { Fourth Princeton/AIAA Conference } \\
\text { (May 1979) }\end{array}\end{array}$ & SOURCE: 2 \\
\hline
\end{tabular}

KEY WORDS: Economics

Energy Source

Industrialization

Habitat Requirements

Manned Flight

Space Colonization 


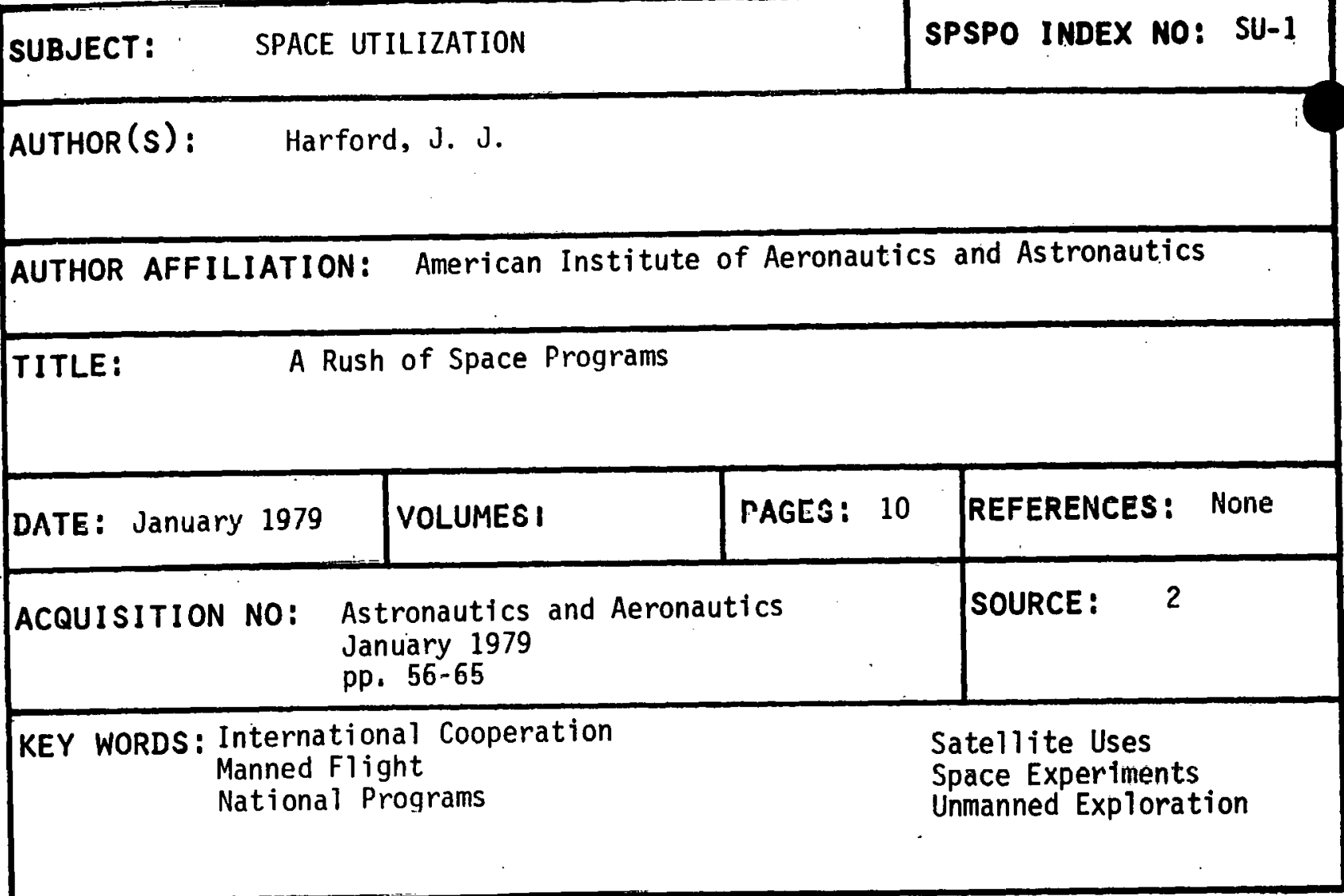

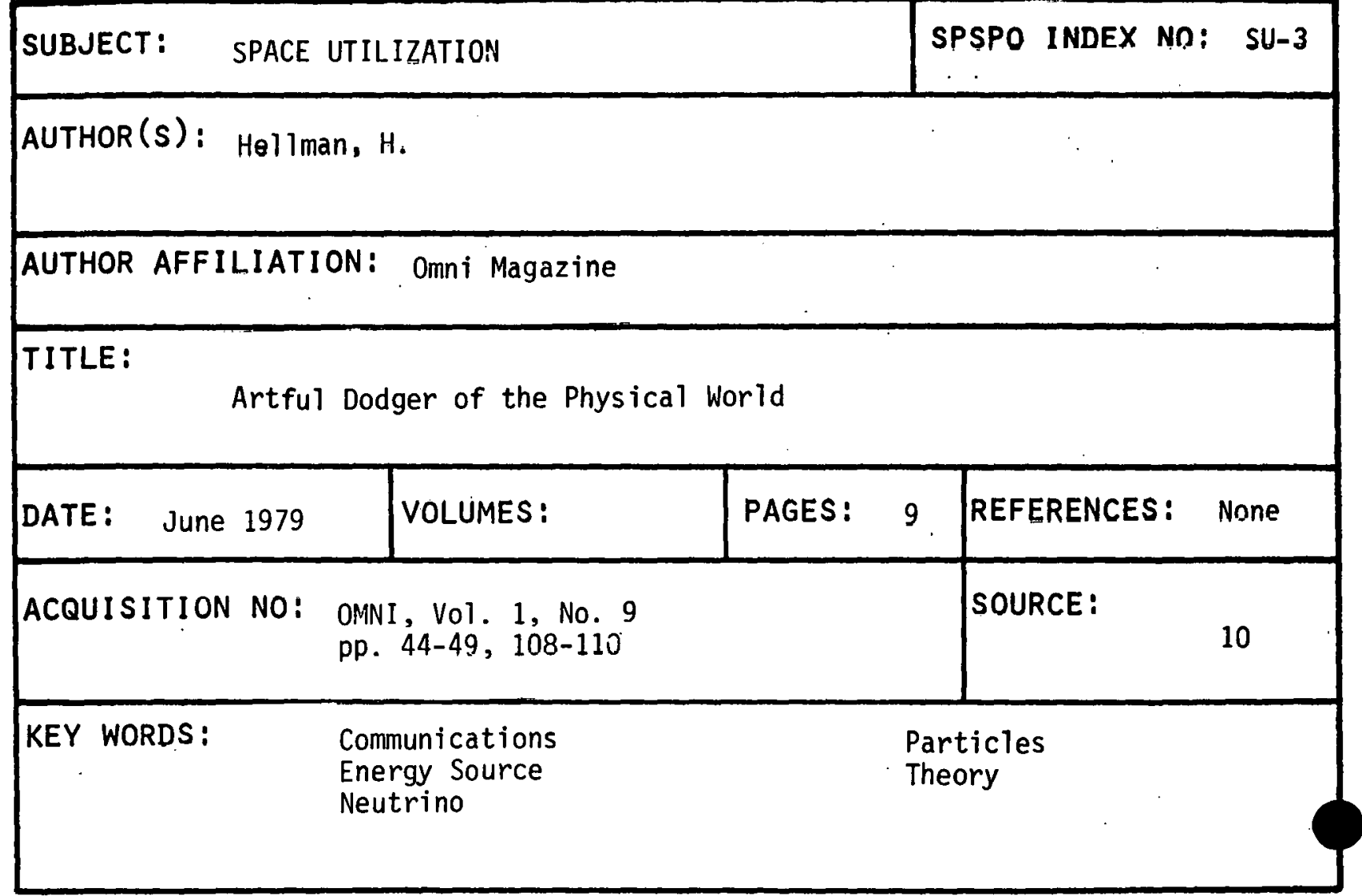


SUBJECT: SPACE UTILIZATION

SPSPO INDEX NO: SU-9

AUTHOR (s): Homatas, G.

AUTHOR AFFILIATION: Polytechnic Institute of New York

TITLE: Man Powered Flight in a Space Habitat.

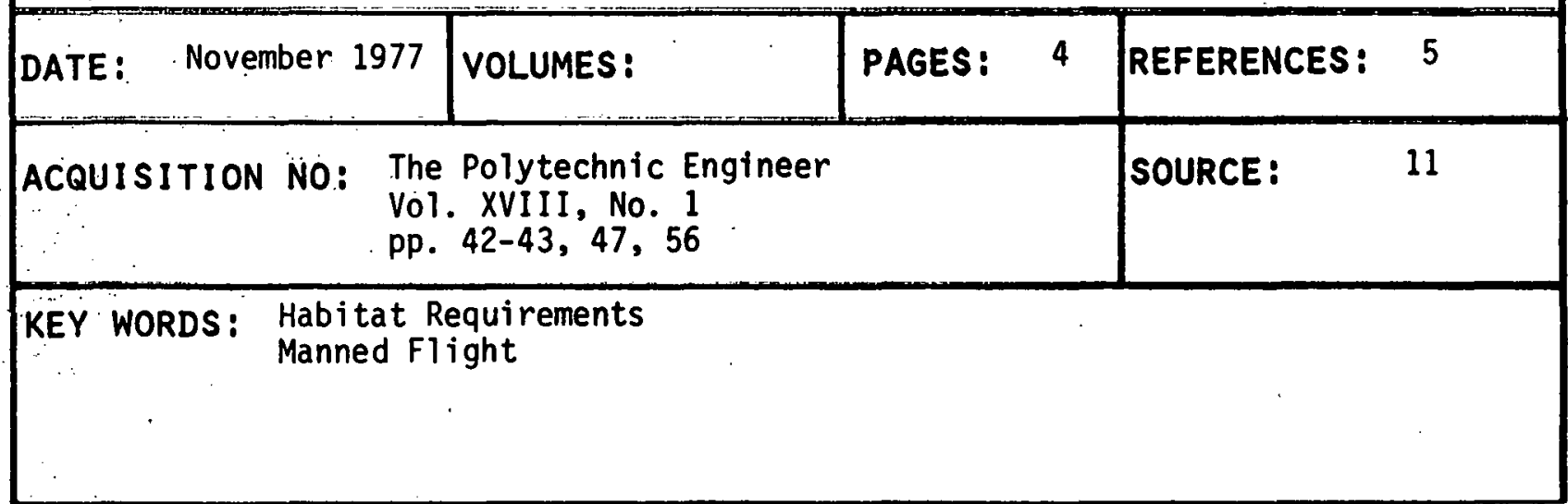

SUBJECT: SPACE UTILIZATION

SPSPO INDEX NO: SU-5

AUTHOR (s): MC Hugh, D.

AUTHOR AFFILIATION: Polytechnic Institute of New York

TITLE: Space Industrialization: Redefining the World

DATE: $\quad$ November 1977 VOLUMES:

\begin{tabular}{l|l} 
PAGES: 14 & REFERENCES: 33
\end{tabular}

ACQUISITION NO: The Polytechnic Engineer

Vol. XVITT, No. 1

pp. $6-17,55-56$

SOURCE:

11

KEY WORDS: Energy Source

Industrialization

Manufacturing 


\section{SUBJECT:}

SPACE UTILIZATION

AUTHOR(s): Marshall Space Flight Center

AUTHOR AFFILIATION: National Aeronautics and Space Administration

TITLE: $\quad$ Space Colonization by the Year 2000 -- An Assessment

\begin{tabular}{|l|l|l|l|}
\hline DATE: January 1975 & VolUMES: & PAGES: 60 & REFERENCES: None \\
\hline ACQUISITION NO: $\quad$ By Title & SOURCE: & 4 \\
\hline KEY WORDS: & $\begin{array}{l}\text { Economics } \\
\text { Habitat Requirements } \\
\text { Space Colonization }\end{array}$ & Space Properties \\
\hline
\end{tabular}

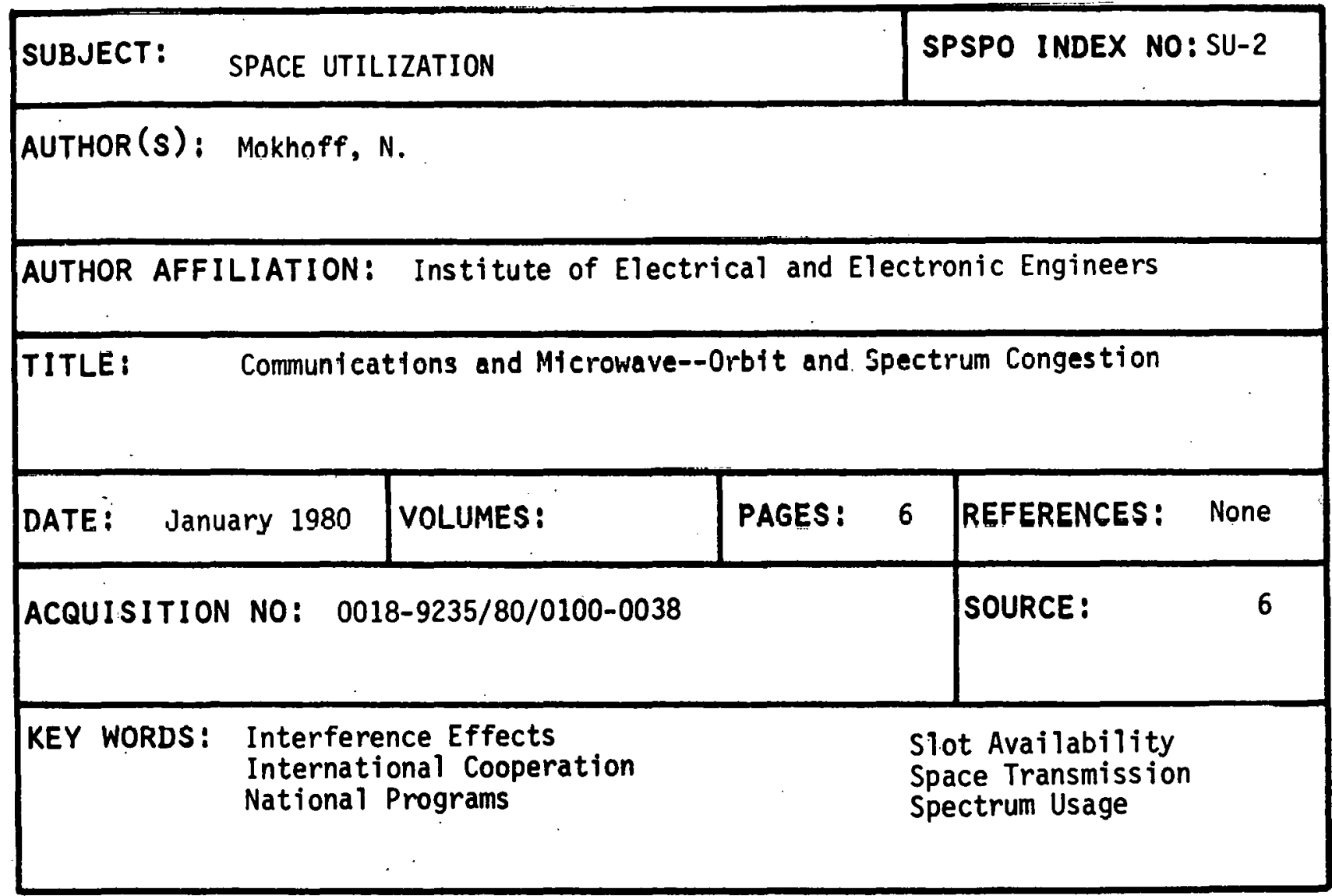


AUTHOR(s): National Aeronautics and Space Administration

AUTHOR AFFILIATION: National Aeronautics and Space Administration

TITLE: A Forecast of Space Technology: 1980-2000

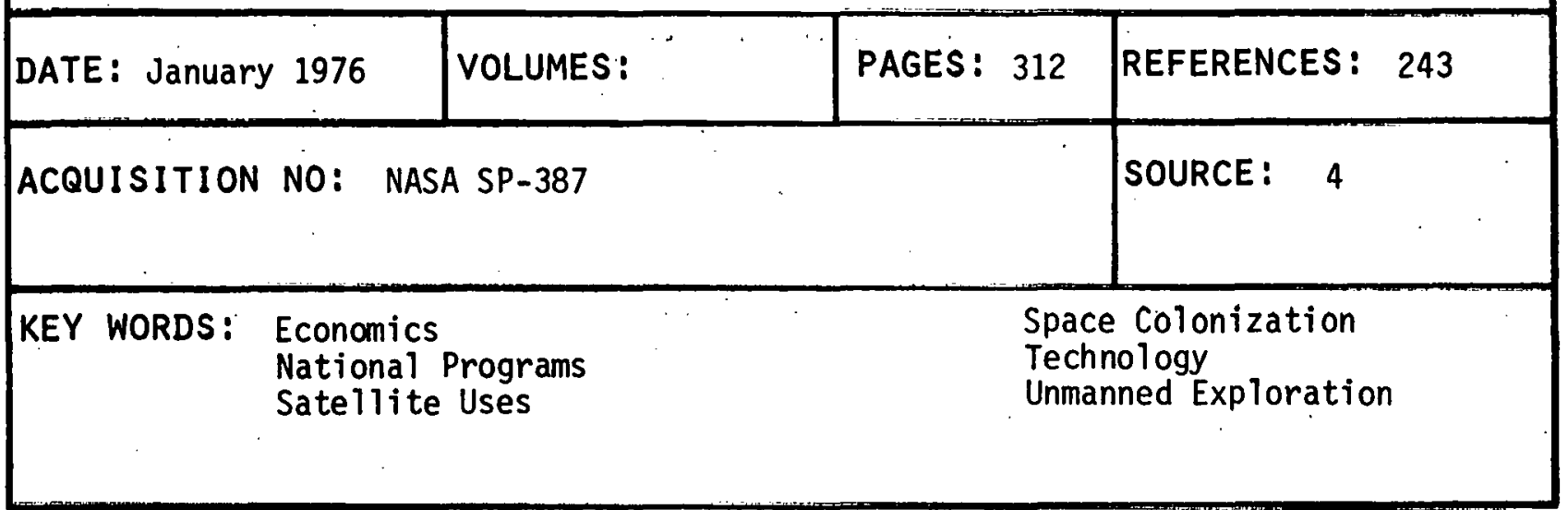

SUBJECT: SPACE UTILIZATIION

SPSPO INDEX NO: SU-16

AUTHOR(s): National Research Council

AUTHOR AFFILIATION: National Academy of Sciences

TITLE: Materials Processing in Space

$$
\text { . }
$$

DATE: 1978

VOLUMES:

PAGES : 75

REFERENCES: None

ACQUISITION NO: By Title

SOURCE :

\section{7}

KEY WORDS: Industrialization

Manufacturing

Material Processes
Space Properties

Space Shuttle 
$\operatorname{AUTHOR}(\mathrm{S})$ : O'Neill, G. K.

AUTHOR AFFILIATION: Princeton University

TITLE: Space Colonies and Energy Supply to Earth

\begin{tabular}{|l|l|l|l|}
\hline DATE: December 1975 & VolumES: & PAGES: 5 & REFERENCES: 23 \\
\hline ACQUISITION NO: By Titte; Vol. 190, pp. $943-947$ & SOURCE: 7 \\
\hline KEY WORDS: $\begin{array}{ll}\text { Economics } \\
\begin{array}{l}\text { Energy Source } \\
\text { Industrialization }\end{array}\end{array}$ & $\begin{array}{l}\text { Manufacturing } \\
\text { Satellite Uses } \\
\text { Space Colonization }\end{array}$ \\
\hline
\end{tabular}

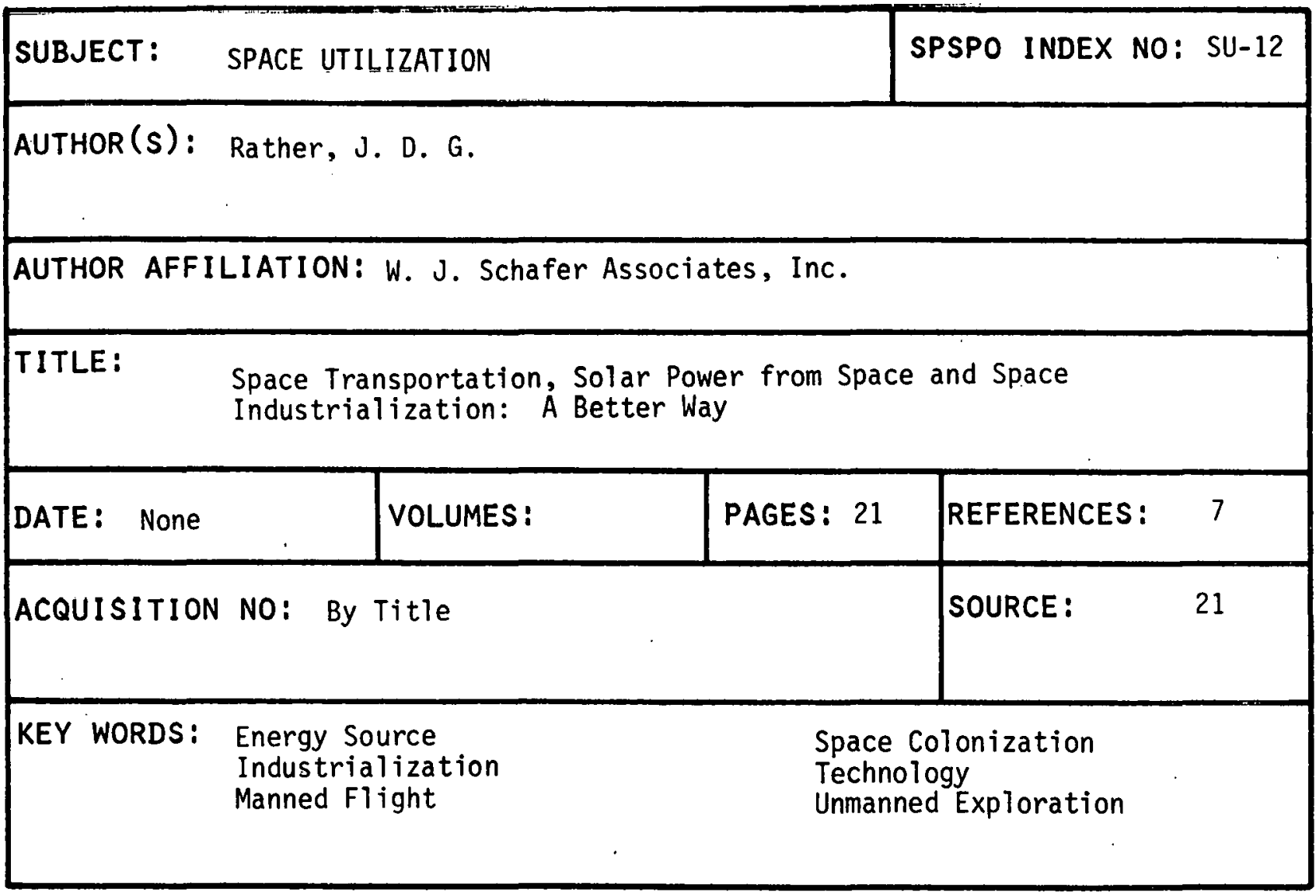




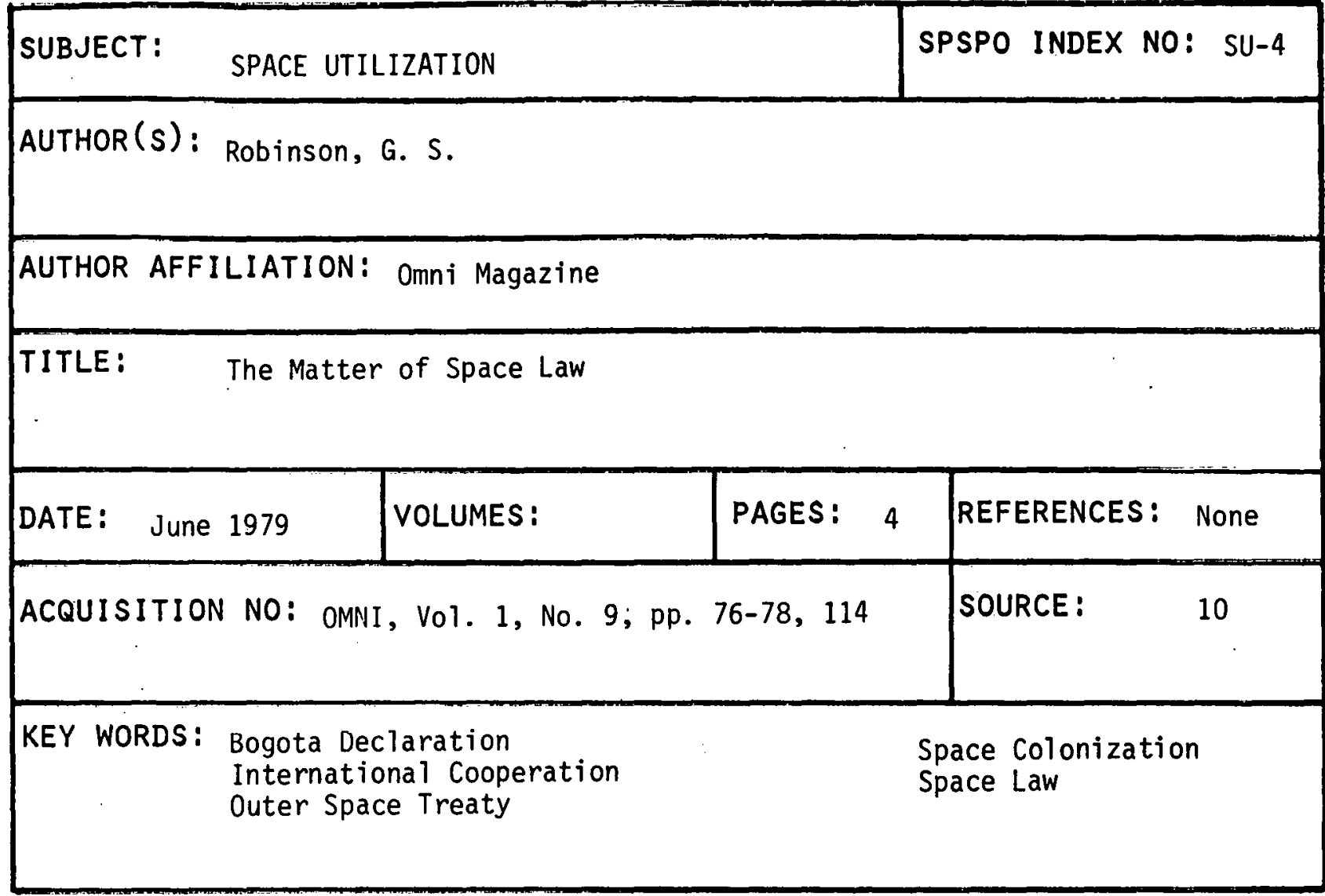

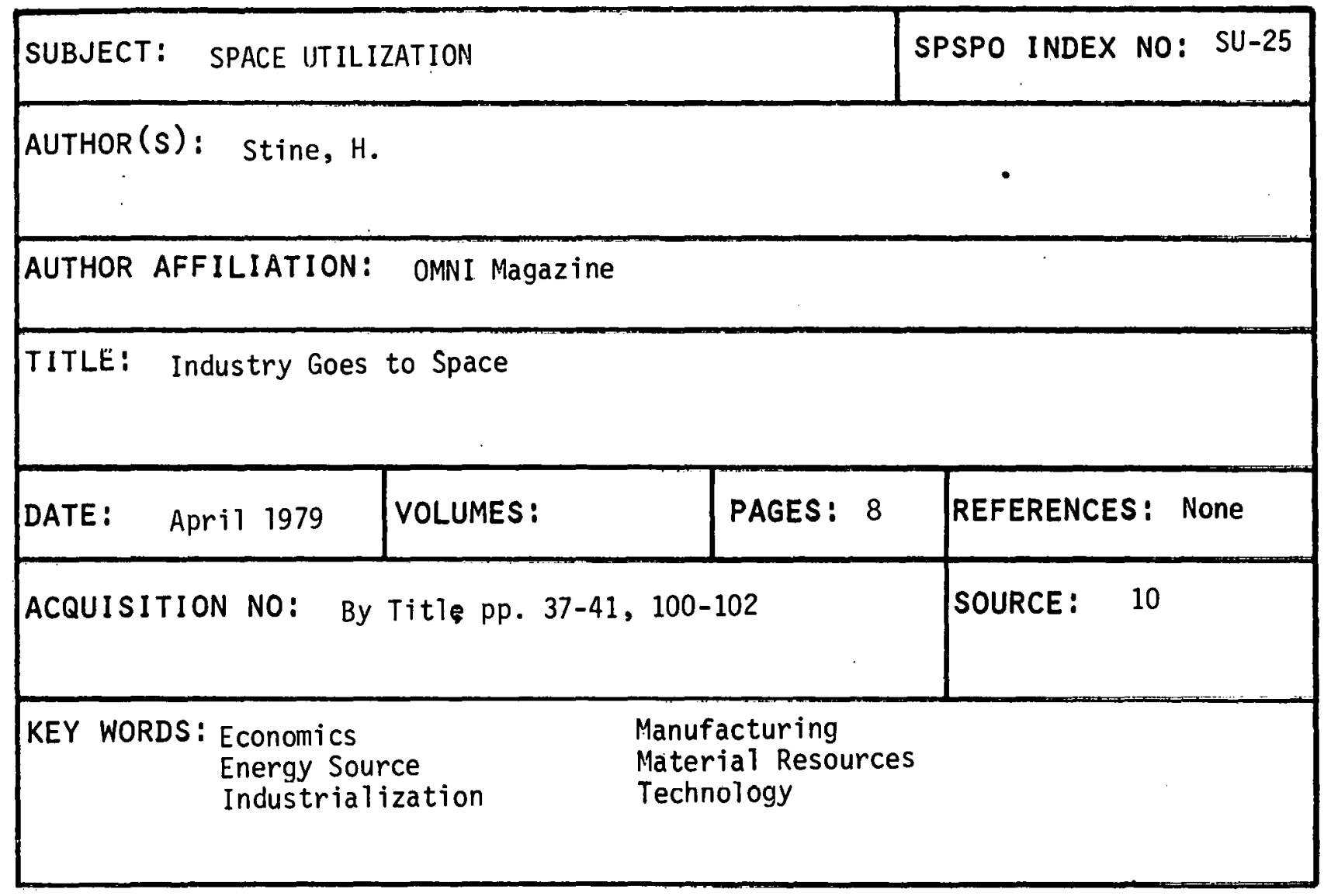


AUTHOR(s): Turner, G. F.

AUTHOR AFFILIATION: Lockheed Missiles and Space Company

TITLE: Electric Power Systems for Space

\begin{tabular}{|c|c|c|c|c|}
\hline DATE: Dccomt & oer 1976 & VOLUMES : & PAGES: 12 & REFERENCES : Nono \\
\hline \multicolumn{4}{|c|}{ ACQUISITION NO: By Title, pp. 18-29 } & SOURCE : 2 \\
\hline \multicolumn{4}{|c|}{$\begin{aligned} \text { KEY WORDS: } & \text { Energy Source } \\
& \text { Satellite Uses } \\
& \text { Technology }\end{aligned}$} & $\cdot$ \\
\hline
\end{tabular}

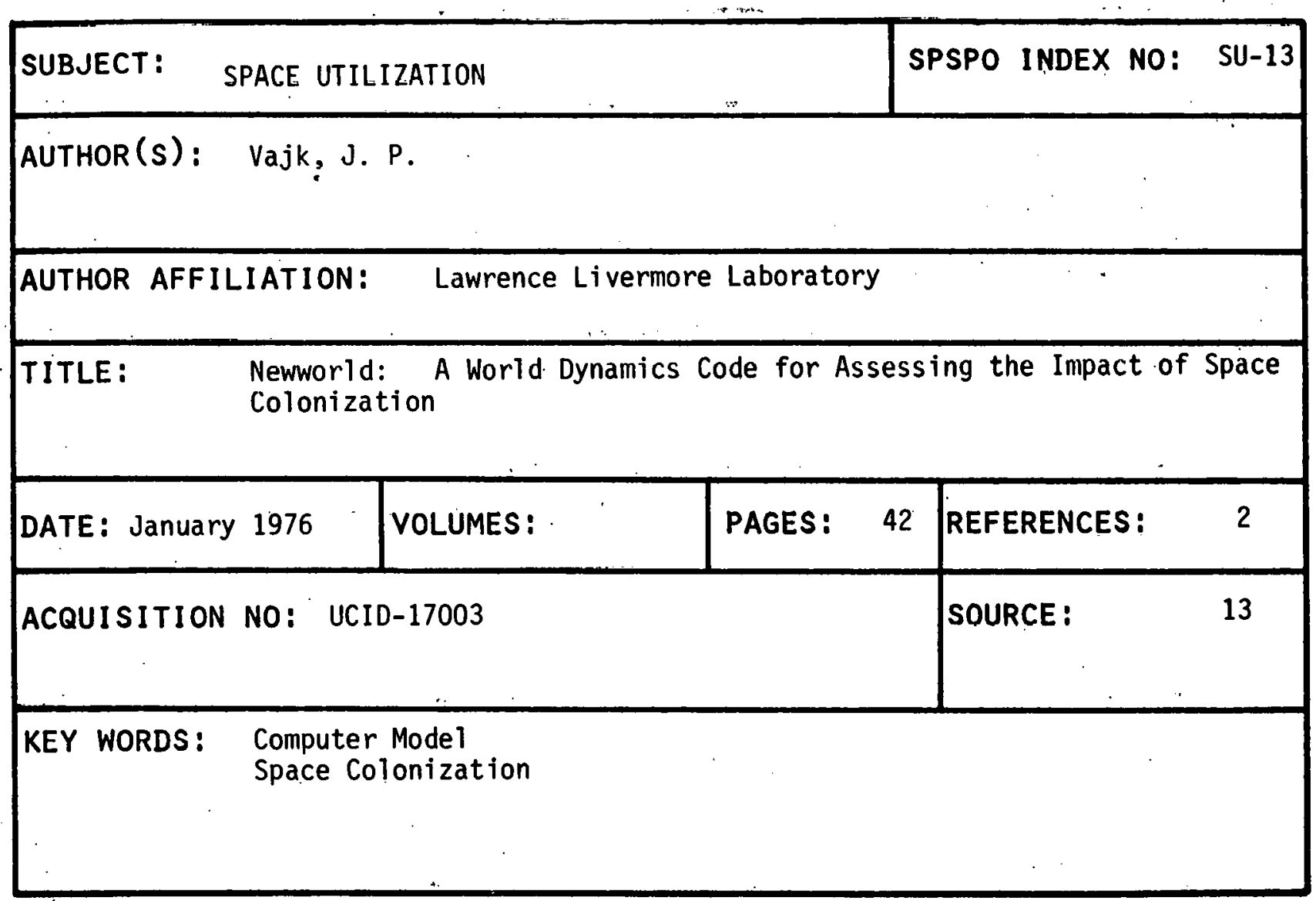




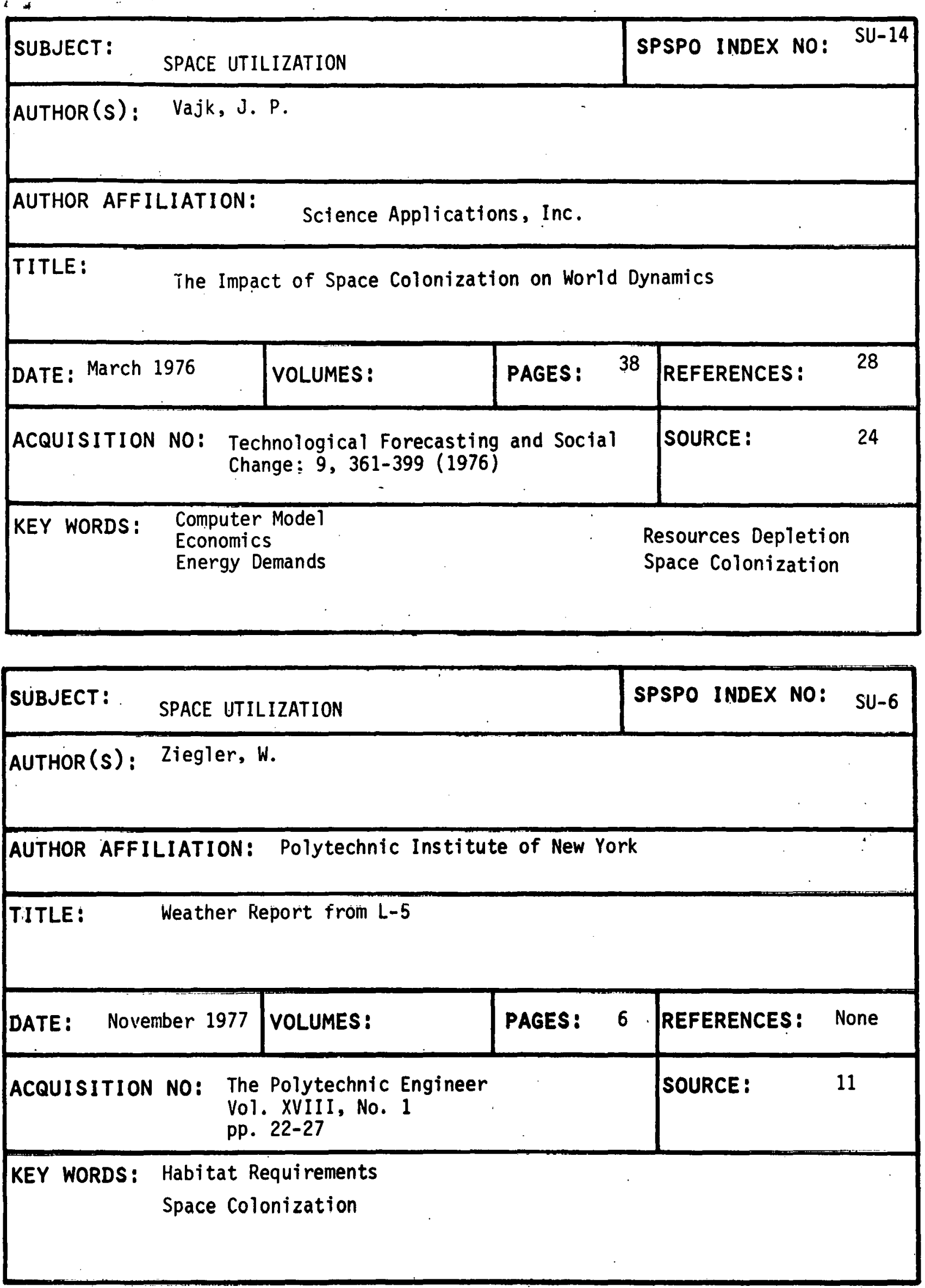




\section{THIS PAGE \\ WAS INTENTIONALLY \\ LEFT BLANK}




\section{AUTHOR INDEX}

A

Abdulezer, L. 225

Abromavage, M. M. 89,99

Adornato, R. J. 158

Aerospace Corporation 111

Alabama, University of $149,175,217$

Allen, K. C. 17

Allen, N. C. 3

Alimgren, D. W. 183

American Institute of Aeronautics and Astronautics 71, 225

Ames Research Center 3

Anderson, B. 81

Andryczyk, R. 175

Argonne National Laboratory 53, 149,176

Arnold, J.R. 54, 226

Arthur D. Little, Incorporated 197

Asbury, J. G. 197

Ayres, R. U. 54

\section{B}

Babcock, W. H. 71

Bachrach, A. 198

Bain, C. N. 121, 198

Baker, L. R. 44, 144

Baldwin, T. E. 199

Baranski, S. 23

Barany, R. 61

Basu, S. 4

Battelle Columbus Laboratories 217

Baver, E. 4

Baumgardner, B. 13

Bavinger, B. A. 141

Behnke, R. A. 10,19
B (continued)

Bekey, , I. 176

Bel1, C. R. 54

Benedick, M. H. ,24

Bernhardt, P. A. 5

Beverly, R. E. 5, 89, 90

Billman, K. W. 122, 150

Blackburn, J. B. 141

Blocker, W. 176

Bloomquist, C. E. 111, 151, 199

Bloomster, C. H. 143, 146

Boeing Aerospace Company 90, 122, $123,151,177,178,218$

Bohn, J. G. 200

Borrego, J. M. 185

Bowen, S. W. 150

Bracken, T. D. 46

Bresters, A. R. 179

Bridges, J. E. 24

Brodeur, P. 25

Bronfman, B. H. 200

Brotzen, F. R. 131

Brown and Root Development, Incorporated 179

Brown, C. D. 62, 68

Brown, W. C. 124, 125; 126, 127, 128, $131,134,152$

Brubaker, K. L. 6, 16

Bruzzone, C. 138

Buehring, W. 67, 105

Buhler, R. D. 219

C

Cahi11, D. F. 94

Cantafio, L.J. 152 
C (continued)

Caputo, R. S. 55

Carasso, M. 61

Carnes, S. A. 200

Carroll, J. C. 17

Carter, N. E. 60

Cassady, P. 138

Chaco, C. C. 6

Chan, C. H. 180

Chapman, P. K. 95

Chernoff, R. C. 128

Chestek, J. 175

Chie, C. M. 180

Chiu, Y. T. 7

Cho, B. S. 56

Chobotov, V. A. 152

Christian, J. E. 211

Christiansen, W. 138

Christol, C. Q. 201

Cirillo, R. R. 56, 63, 67, 68

Citizens' Energy Project 201, 202

Clark, K. M. 97

Claverie, M. J. 56, 57

Clearly, S. F. 25

Coates, V. T. 57

Collins, D. S. 182

Collins, P. Q. 181

Colson, W. B. 130,131

Committee on Commerce, Science and Transportation 26

Cormittee on Governmental Affairs 27

Community and Economic Development Division 27

Comptroller General of the U.S. 28

Conley, L. 105
C (continued)

Cook; H. J. 48

Cooke, D. L. 191

Crist, M. 202

Criswell, D. R. 7, 8, 203, 226

Crowley, J. H. 58

Csigi, K. 183

Culver, D. H. 141

Curtis, S. B. 28

Czerski, P. 23

D

da Rosa, A. V. 5

Daurio, A. P. 199, 203

David, L. 29, 209

Davis, K. A. 81,94

Dawley, R. 76

De, B. R. 7, 8

Deffeyes, K. S. 35

DeForest, S. 8

Dickinson, R. M. 91, 129

Doane, J. W. 58

Donalek, P. J. 181

Douglas, M. 8

Drucker, H. 59

Duffy-Armstrong, M. 204

Duke, M. B. 226

Dula, A. 29

Duncan, L. M. 9, 10, 11, 19

Dunn, B. D. 182

Dupas, A, P, 56, 57 


\section{AUTHOR INDEX}

E

Eberhardt, A. W. 142

Econ, Incorporated 103

Edler, H. G. 204

Edwards, A. 134

Ehrenreich, H. 142

Ekstron, P. A. 82

Electromagnetic Radiation Management Advisory Council 30, 31, 32

Eltgrowth, M. W. 14

Emerle, E. 34

Energy, U.S. Department of 91,92 , $112,113,114,153,182,205$

Energy Analysis, Institute for 62

Energy Research and Development Administration $59,72,92,93$, 115,154

Enk, G. 200

Environmental Health

Directorate 32,33

Environmental Protection Agency 33

Environmental Resource Group 94

Espeland, R. H. 17

$F$

Fitzsimmons, G. W. 130

Foldes, P. 175

Fordyce, S. W. 131

Formanek, V. C. 34

Forum for the Advancement of Students in Science and

Technology, Inc. 205

Fraley, D. W. 60

Freedman, R. S. 227

Freeman, J. W. 130, 131, 191

Fujita, T. 60,61
G

Gallagher, J. M. 61

Galloway, E. 227

Gandhi, O. P. 34

Gary, N. N. 23

Gasper, J. R. 62, 67, 68 .

Gatzke, A. E. 98

Gaudet, A. D. 183

Gauger, J. R. 34

Gehrig, J. J. 228

General Accounting office 72

George Washington University 206

Gerlach, L. P. 207

Gerry, E. T. 135

Gibson, M. H. 132

Gilbert, R. 0.46

Gilbreath, W. P. 150

Glaser, P. E. $73,95,154,155,156$, $157,158,183,203$

Goldford, A. I. 10

Gordon, W. E. 9, 11

Gordon, Z. V. 35

Gorman, D. 184

Gorman, R. 76

Gorove, S. 207, 228

Gould, J. L. 35, 36

Grant, C. 184

Grant, W. B. 81,83

Greenstein, D. 211

Grey, J. 73, 229

Grumman Aerospace Corporation 184

Gurwe 11, W. E. 143, 146

Guttman, R. J. 185

Guy, A. W. 36,40 


\section{AUTHOR INDEX}

H

Habegger, L. J. $62,67,68$.

Hagler, H. 211

Haley, J.T. 134

Halverson, S. L. 94

Ham, W. T. 37

Hanley, G. M. 158, 186, 187

Harford, J. J. 230

Hartley, J. N. 143

Hazelrigg, G. A. 104, 105, 158, 212

Hazzard, D. G. 37

Hegg, D. A. 14

Hellman, H. 230

Helms, I. L. 218

Herendeen, R. A. 74

Hermiter, B. . 13

Hertzberg, A. 219

Hester, J. 131

Hill, J. 202

Hill, L. G. 199

Hinds, W. T. 40

Hjeresen, D. L. 40

Hobbs, P. V.. 14

Holdren, J. P. 66

Holt, B. R. 97

Homatas, G. 231

Howe 11, J. M. 134

Hub, K. 67, 68, 105

Huie, H. H. 188

Hung, J. C. 188.

Hunter, M. W. 160

I

International Radiation Protection Association 38
I (continued)

Ishimaru, A. 38

J

Jackson, W. L. 211

Jamieson, W. M.: 146

Jet Propulsion Laboratory 189

Johnson, A. L. 4

Johnson, C. C. 39

Johnson, U. E. 106

Johnson, R. W. 189

Johnson Space Center 11, 39, 159, 160

Jones, D. C. 97

Jones, W. C. $1.60,219$

Juroshek, J. R. 81

Justesen, D. R. 40

K

Kane, G. L. 48

Kantak, A. V. 180

Kaplan, S. D. 97

Kary T. 74

Kassing, - D. 75

Kaupang, B. M. 175

Kellermeyer; D. A. 63

Kemp; N. H. 221

Kierolff, H. E. 106

Kirschvink, J:L. 35

Kline, R. L. 157

Klineberg, S. I. 208

Klobuchar, J. A. 4

Koomanoff, F. A. 161, 162

Koppelman, L. 211

Kornberg, J. P. 95

Kotin, A. D. 143, 202, 209 


\section{AUTHOR INDEX}

$K$ (continued)

Kozakoff, D. J. 132

Kraft, C. C. 163

Kramer, P. R. 219

Krebs, J. R. .97

Krop, C. 229

Kuo, T. J. 44, 144

Kyrias, G. 184

L

Ladwig, A. 209

LaQuey, R. 8

Lawrenece Berkeley Laboratory 41

Lee, J. L. 12,16

Lee, J. M. 46

Leininger, G. 137

Leon, H. I. 76

Levine, E. P. $56,63,68$

Levinson, R. J. 75

Liebe, H. J. 82

Lindsay, T. A. 190

Lindsey, C. 8

Lindsey, W. C. 180

Litchfield, J. W. 143

Livingston, F. R. 95, 144

Livingston, L. E. 96

Lockheed Missiles and Space Company 133, 220

Logsdon, J. M. 115

Lopukhin, V. M. 169

Lord. . $C$. 184

Love11, B. 81

Luhmann; J. G. 7
Mac - Mc

MacDonald, G. J. 47

McCreary, S. T. 98

McDonald, C. L. 60

McDougal1, J. A. 36

Mctugh, D. 231

McNeal, S. R. 210

McRae, M. V. 164

M

Machol, R. E. 76

Mackovciak, J. 155

Mallalieu, R. C. 41

Markson, R. 12

Marsh and McLennen, Incorporated 210

Marshall Space Flight Center 164, 232

Martin, J. H. 142

Massey, K. A. 42

Mathematical Sciences Northwest, Incorporated 133

Maynard, O. E. 124, 134, 155

Mayur, R. 203

Meltz, G. 134

Mendi110, M. 6, 13

Michaelson, S. M. 42

Mixon, W. R. 211

Mokhoff, N. 232

Monarch, M. R. 56

Morgan, M. G. 46

Morrison, E. L. 81,83

Moses, H. 165

Mueller, R. 0. 197

Muir, M. 12

Myers, D. H. 77 


\section{AUTHOR INDEX}

N

Naisbitt, J. 211

Nalos, E. J. $130,135,190$

Nansen, R. H. 106, 157

Nathan, A. 134

National Aeronautics and Space Ad-

ministration $112,115,165,182,233$

National Research Councif 43, 83, $-166,-233$

National Space Institute 212

National Telecomunications

Information Administration 14,43

Neice, S. 138

Nelson, L. 138

Nelson, T. A. 146

Newsom, B. D. 116

Newsom, D. E. 64, 67, 68

0

O'Leary, B. T. 203

O'Neill, G. K. 203, 234

Ostrowski, K. 44

O'Tuule, R. P. 58

Ott, R. H. 83

Ozeroff, M. J. 212

Ozerski, P. 44

P

Pacific Northwest Laboratory 84

Patmore, J. W. 200

Pawlewicz, W. W. 146

Pearsons, K. S. 96

Peebles, P. Z. 188

Peelle, E. 200

Phillips, R. D. 40
$P$ (continued)

Phillipson, L. L. 111

Piland, R. 0. 163

Pindroh, A.: 138

Pine, G. D. 211

Pirri, A. N. 221

Poch, L. A. 97, 213

Pollacsek, M: 132

Polson, P. 97

Poon, P. T. Y. 44, 144

PRC Energy Analys is Company 213

Price, K. M. 5

Q

Quimby, D. 138

R

Rabe," J. A. 202

Radiation Protection Bureau 45

Radkc, L. F. 14

Ragan, H. A. 40

Ralph, E. L. 155

Rather, J. D. G. 135, 234

Raytheon Company 190

Rebitzer, 1. 74

Reeber, R. R. 144

Reiff, P. H. 191

Reilly, J. P: 136

Reinhartz, K. K. 75

Repacholi, M. H. 45

Rice, E. E. 220

Rice University 15,145,166, 191, 192

Ridker, R. G. 54

Rieck, T. A. 107 
$R$ (continued)

Rish, W. R. 46

Ritschard, R. L. 214

Robinsoñ, G. S. 235

Rockwell International 136, 166, 167

Rogers, L. E. 40,46

Romback, J. 184

Rosi, F, O. 145

Rossin, A. D. 107

Rote, D. M. 13, 15, 16, 94

Rowland, B. 105

Ruderman, M. A. 47

Rush, C. M. 4, 17, 94

Ruth, J. 214

\section{S}

Salis, M. 184

Samsa, M. E. 64, 67, 68

Sandah1, C. A. 161, 162

Santini, D. J. 199

Savvin, V. L. 169

Schiefelbein, S. 47

Schimmerling, W. 28

Scholtz, R. A. 180

Schuchardt, J. M. 132

Schuh, R. 137

Schulz, M. 7

Schwe1tzer, M. 200

Schwenk, F, C. 168

Scott-Walton, B. 97

Selenk, M. K. 65, 66

Senew, M. J. 63

Shanker, R. 211

Shepherd, R. A. 97
$S$ (continued)

Shore, M. L. 39, 44

Shotland, S." 199.

Showen, R. L.: 17

Siegel, S. B. 71

Silverman, C. 44

Simons, S. 130, 131

Skidmore, R. 184

S7iney, D. H. 37

Smith, K. R. 66

Smith, M. S. 77

Smith, P. L. 98

Smith, S. A. 146

Spaulding, A. D. 84

SRI International 98

Steneck, N. H. 48

Stenehjem, E. J. 67, 199

Stephenson, M. 105

Stevens, G. H. 137

Stine, H. 235

Stokes, G. M. 82

Stone, S. N. 19

Strijk, S. 132

Suess, M. J. 44

Sun, K. 219

Sutherland, C. D. 18, 19

Sviedrys, R. 78

Swanson, C. A. 71

T

Tanzman, E. 68

Taussig, R. 138

Tayi, G. 54

Taylor, R. B. 192 


\section{AUTHOR INDEX}

$T$ (continued)

Technology Assessment, Office of 168

Teeter, R. R. 141, 146

Telecommunications Sciences, Institute of 85

Tennessee, University of 193

Thomas, R. 184

TRW Energy Systems Planning Division 67

Turner, G. F. 236

U

Universities Space Research Association 116

v

Vajk, J. P: $107,203,236,237$

Valentino, A. R. $16,48,89 ; 99$

Vander, A. J. 48

Vanke, V. A. 169

Van Kuiken, J. 68

Violette, E. J. 17

W

Walbridge, E. W. 138

Waldeskog, B. 44

Warshaw, H. 8

Watson, W. D. 54

Watts, J. W. 49

Watts, R. L. 143, 146

Webb, M.'D. 36

Wei, M. S. 1.17

Weiss, R. F. 221
$W$ (continued)

Wendel1, C. A. 158

Westerdah1, B. B. 23

Westphal, $W .214$

Weyant, J. 66

White, M. $49,94,100$

White, P. H. 96

Whitfield, R. 67, 68

Whysong, I. L. 181

Wilhy, 1, F, 96

Wolfe, M. G. $152,169,222$

Wolsko,' T. D. $64,68,97,213$

Woodcock, G. R. 108, 170, 171, 221

Wright, J. J. 49

\section{$x$}

$Y$

Yamaura, I. 50

Young, J. R. 97

2

Zaininger, H. W. 200

Zeiders, G. W. 135

Ziegler, E. J. 58

Zlegler, W. 237

Zimmerman, R. G, 61

Zinn, J. 9, 18, 19 


\section{ACQUISITION SOURCES}

1. ESA SPACE DOCUMENTATION SERVICE 8-10, RUE MARIU NIKIS

75738 PARIS CEDEX 15

FRANCE:

2. AMMERICAN INSTTITUTE OF AERONAUTICS AND ASTRONAUTICS 1290 AVENUE OF THE AMERICAS

NEW YORK, NY 10019

3. CONGRESSIONAL RESEARCH SERVICE LIBRARY OF CONGRESS WASHINGTON, D. C. 20540

4. SCIENTIFIC AND TECHNICAL INFORMATION OFFICE NATIONAL AERONAUTICS AND SPACE ADMINISTRATION WASHINGTON, D.C. 20546

5. NATIONAL TECHNICAL INFORMATION SERVICE' U.S. DEPARTMENT OF COMMERCE SPRINGFIELD, VA 22161

6. INSTITUTE OF ELECTRICAL AND ELECTRONICS ENGINEERS 445 HOES LANE PISCATAWAY, NJ 08854

7. AMERICAN ASSOCIATION FOR THE ADVANCEMENT OF SCIENCE 1515 MASSACHUSETTS AVENUE, N.W. WASHINGTON, D.C. 20005

8. AMERICAN BAR ASSOCIATION SCIENCE \& TECHNOLOGY DIVISION 1155 EAST 60TH STREET CHICAGO, ILL 60637

9. U.S. GENERAL ACCOUNTING OFFICE REPORTS AND DOCUMENTS BOX 6015

GAITHERSBURG, MD 20760 
10. OMNI PUBLICATIONS INTERMATIONAL, LTD.

909 THIRD AVENUE

NEW YORK, NY 10022

11. POLYTECHNIC INSTITUTE OF NEW YORK

333 JAY STREET

BROOKLYN, NY 11201

12. AMERICAN SOCIETY OF INTERNATIONAL LAW

2223 MASSACHUSETTS AVENUE, N.W.

WASHINGTON, D.C. 20008

13. TECHNICÁL INFORMATION CENTER

P. 0 . BOX 62

OAK RIDGE, TN 37830

14. DUKE UNIVERSITY

SCHOOL OF LAW

DURHAM, NC 27706

15. SATURDAY REVIEW

1290 AVENUE OF THE AMERICAS

NEW YORK, NY 10019.

16. RADIATION PROTECTION BUREAU

DEPARTMENT OF HEALTH AND WELFARE

BROOKFIELD ROAD

FEDERATION HEIGHTS

OTTANA, ONTARIO CANADA KIAICI

17. DEPARTMENT OF BIOPHYSICS

VIRGINIA COMMONWEALTH UNIVERSITY

RICHMOND, VA 23298

18: LUNAR AND PLANETARY INSTITUTE

3303 NASA ROAD 1

HOUSTON, TX 77058

19. UNIVERSITIES SPACE RESEARCH ASSOCIATION

P. U. BOX 1892

HOUSTON, TX 77001

20. SUPERINTENDENT OF DOCUMENTS

U.S. GOVERNMENT PRIRTING OFFICE

WASHINGTON, D.C. 20402 
21. W. J. SCHAFER ASSOCIATES, INC.

1901 NORTH FT. MYER DRIVE, SUITE 803

ARLINGTON, VA 22209

22. COMMISSIONER OF PATENTS

U. S. PATENT OFFICE

WASHINGTON, D. C. 20231

23. DOWDEN, HUTCHINSON AND ROSS, INC.

STROUDSBURG, PA 18360

24. AMERICAN ELSEVIER PUBLISHING COMPANY, INC.

52 VANDERBILT AVENUE

NEW YORK, NY 10017

25. AMERICAN CHEMICAL SOCIETY

1530 P STREET, N.W.

WASHINGTON, D.C. 20005

26. ELECTRIC POWER RESEARCH INSTITUTE

P. 0. BOX 10412

PALO ALTO, CA 94303

27. NATIONAL ACADEMY OF SCIENCES

2101 CONSTITUTION AVENUE, N.W.

WASHINGTON, D. C. 20418

28. ENERGY RESEARCH GROUP

OFFICE OF THE VICE-CHANCELLOR FOR RESEARCH

UNIVERSITY OF ILLINOIS

URBANA, IL 61801

29. TECHNOLOGY REVIEW

ROOM 10-140

MASSACHUSETTS INSTITUTE OF TECHNOLOGY

CAMBRIDGE, MA 02139 
30. AAS PUBLICATIONS OFFICE

P. 0. BOX 28130

SAN DIEGO, CA 92128

31. CITIZENS' ENERGY PROJECT

1110 SIXTH STREET, N.W.

WASHINGTON, D. C. 20001

32. W. W. NORTON AND CO,, INC.

500 FIFTH AVENUE

NEW YORK, N. Y, 10036

33. PERGAMON PRESS

MAXWELL HOUSE

FAIRVIEN PARK

ELMSFORD, N. Y, 10523

34. POLISH MEDICAL PUBLISHERS

WARSAW, POLAND

35. SIGMA XI

THE AMERICAN SCIENTIST

345 WHITNEY AVE.

NEM HAVEN, CT. 06511

36. AMERICAN GEOPHYSICAL UNION 2000 FLORIDA AVENUE, N. H. WASHINGTON, D.C. 20009

37. HEMISPHERE PUBLISHING CORP. GLOBAL BUILDING

1025 VERMONT AVENUE, N. W. WASHINGTON, D.C. 20005

38. FASST, INC.

2030 M. STREET, N. W.

SUITE 402

WASHINGTON, D.C. 20036 
39. INTERNATIONAL MICROWAVE POWER INSTITUTE BOX 1556

EDMONTON, ALBERTA

T5J 2N7 CANADA

40. ACADEMIC PRESS

111 FIFTH AVENUE

NEW YORK, N, Y, 10003

41. AMERICAN INSTITUTE OF PHYSICS

335 EAST 45TH STREET

NEW YORK, N, Y, 10017

42. INTERNATIONAL ACADEMY OF ASTRONAUTICS

250 RUE SAINT-JACQUES

75005 PARIS, FRANCE 


\section{THIS PAGE \\ WAS INTENTIONALLY \\ LEFT BLANK}


The copyright laws of the United States prohibits the reproduction of material published by commercial printers and distributors without the written consent of the originator, including professional organizations such as the American Institute of Aeronautics and Astronautics and the Institute of Electrical and Electronics Engineers. The copyright laws do not apply to reports prepared by or for the U.S. government, and they may be copied without consent. Articles and professional papers should be requested from publishers listed on citation cards. Order forms follow for requesting government documents from the U.S. Government Printing. Office (Source 20), the Department of Energy Technical Information Center (Source 13), and the National Technical Information Service (Source 5). The latter form also should be used for obtaining documents from the NASA Scientific and Technical Information office (Source 4). The following price schedule can be used to estimate costs of purchases from these services.

\begin{tabular}{|c|c|c|c|c|c|}
\hline Page Range & $\begin{array}{c}\text { Domestic } \\
\text { Price }\end{array}$ & $\begin{array}{c}\text { NTIS } \\
\text { Price Code }\end{array}$ & Page Range & $\begin{array}{l}\text { Domestic } \\
\text { Price }\end{array}$ & $\begin{array}{c}\text { NTIS } \\
\text { Price Code }\end{array}$ \\
\hline $001-025$ & $\$ 1.00$ & A02 & $336-3 \overline{5} 0$ &.$\$ 12.00$ & $\triangle 15$ \\
\hline $026-050$ & 4.50 & A03 & $351-375$ & 12.50 & $A 16$ \\
\hline $051-075$ & 5.25 & A04 & $376-.100$ & 13.00 & $A 17$ \\
\hline $076-100$ & 6.00 & A05 & $.401-425$ & 13.25 & +18 \\
\hline $101-125$ & 6.50 & 106 & $+26-150$ & 14.00 & $A 19$ \\
\hline $126-150$ & 7.25 & A07 & $451-475$ & $1+.50$ & +20 \\
\hline $151-175$ & 8.00 & t08 & $476-500$ & $15: 00$ & $A 21$ \\
\hline $176-200$ & 9.00 & .109 & $501-525$ & 15.25 & .122 \\
\hline $201-225$ & 9.25 & $A 10$ & $526-550$ & 15.50 & 123 \\
\hline $.226-250$ & 9.50 & $A 11$ & $551-575$ & 16.25 & $A 2 \cdot 1$ \\
\hline $251-275$ & 10.75 & $A 12$ & $576-600$ & 16.50 & $A 25$ \\
\hline $276-300$ & 11.00 & $A 13$ & 601-up & $\dagger$ & \\
\hline $301-325$ & 11.75 & $A 14$ & . & & \\
\hline
\end{tabular}

* All microfiche are priced $A 01$.

†Add $\$ 2.50$ for each additional 100 page increment from 601 to 1.000 pages. 


\section{THIS PAGE}

\section{WAS INTENTIONALLY \\ LEFT BLANK}




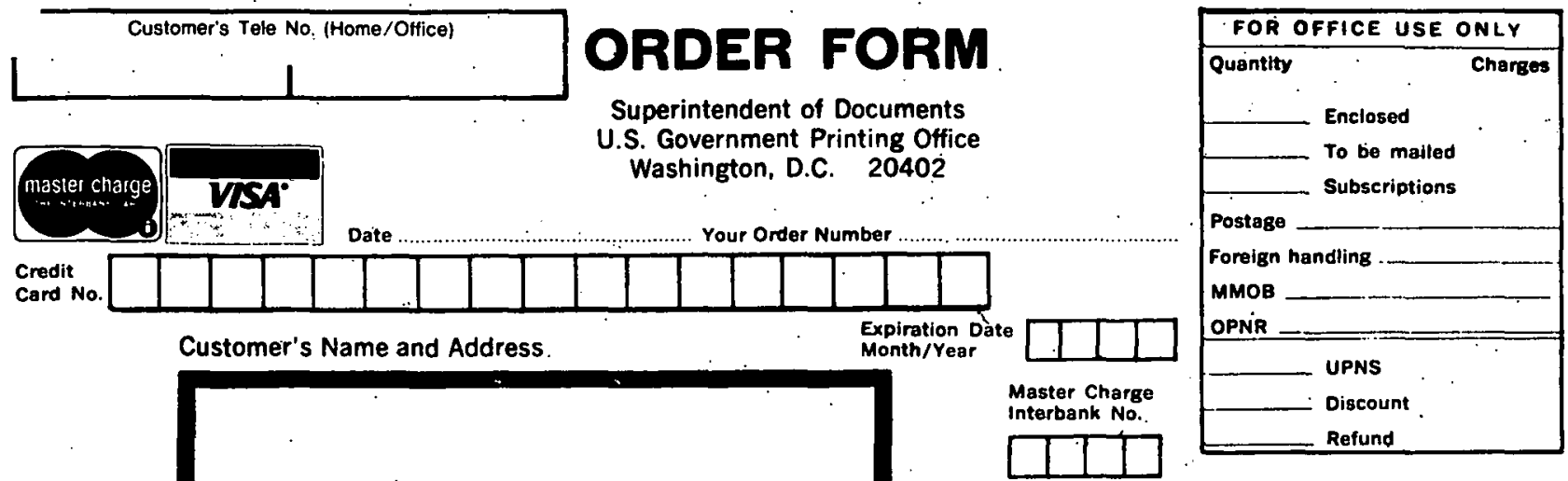

Deposit Account Number

ZIP

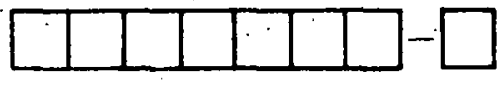

GEFORE USING THIS FORM READ IMPORTANT INFORMATION ON REVERSE SIDE PLEASE PRINT OR TYPE ALL INFORMATION ON THIS FORM.

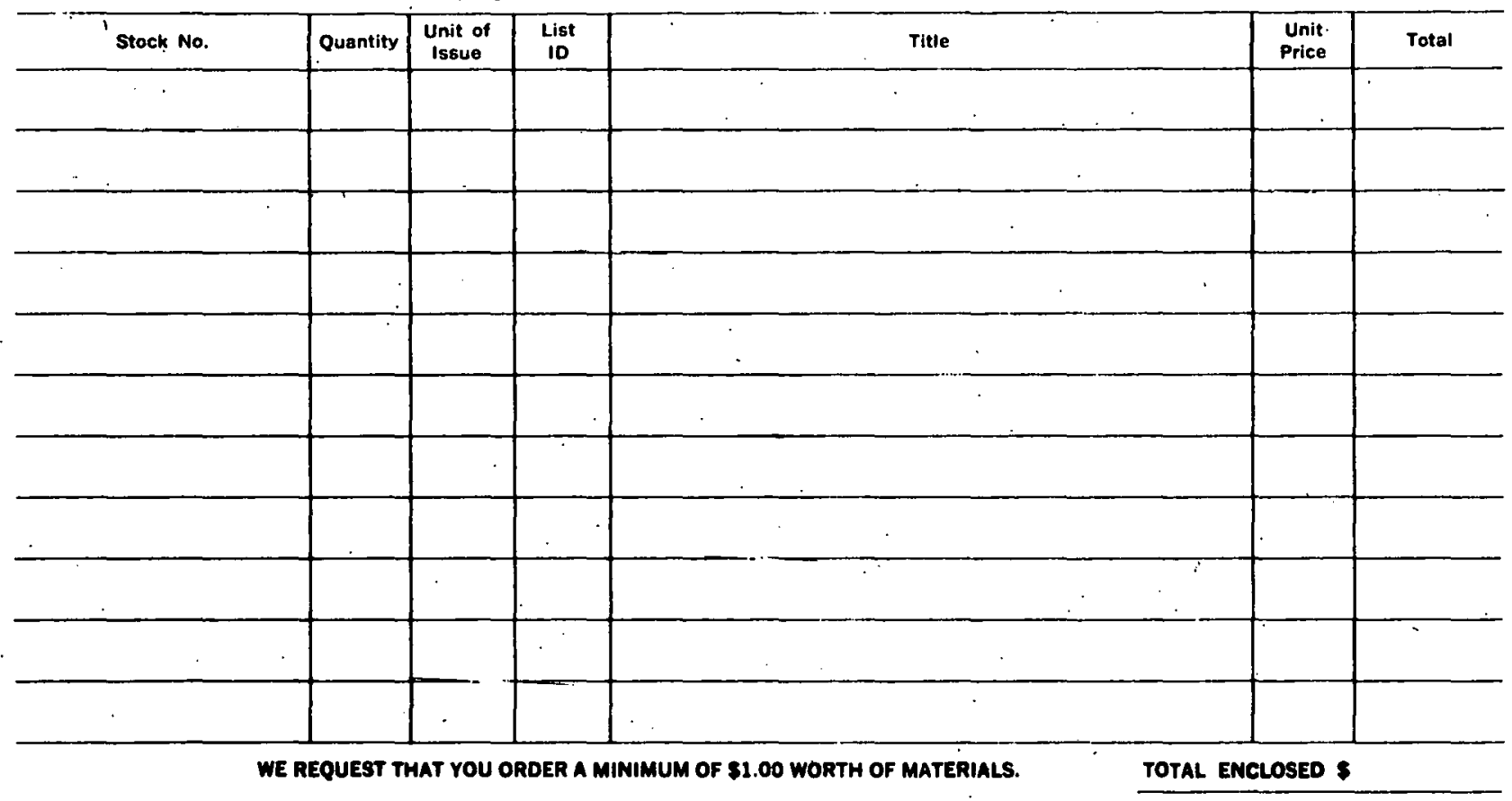

SHIP TO: (it different from abow)

Unit of Issue

PK Package containing muttipli coples

EA. Each-aingle copy

PO Pad containing multiple shoots

SE Sot of multiple Items

Kit of multiple items in a spocial container 


\section{INFORMATION CONCERNING YOUR ORDER OR INQUIRY}

Thank you for your interest in U.S. Government publications. If you have made a written inquiry, it is returned herewith for your reference. We have indicated the prices of publications currently available, or have provided the information requested to the best of our ability. Uniess otherwise noted, prices are for single copies.

Regulations require payment in advance of shipment of publications. Please make checks or money orders payable to the Superintendent of Documents. You may charge your order using your Deposit Account, Master Charge, or Visa. Do not send currency (bills or coins) or postage stamps.

Shipping is by non-priority mail or United Parcel Service. First slass mailing is available at a higher rate. Contact us for rates if you desire this service. (202-783-3238)

With the exception of sperially priced publications and subscriptions, a discount of 25 percent is allowed on quantity purchases (100 or more copies of a single publication): The same discount applies to orde's from $\dot{a}$ bookdealer (any quantity) when the publications are mailed to the dealer's normal place of business. No discount will be allowed when publications and subscriptions are mailed to a third party (unless in quantities of 100 or more per title).

NON U.S. CUSTOMERS: International mailing regulations require special handling for which wa oharge ail additional $\mathbf{2 5}$ percent of the total cost of your order. Remittann.e is requirod in advalice of shipping by draft on a United States or Canadian bank, by UNESCO coupons. or by international Postal Money Order made payable to the Superintendent of Documents. These orders are mailed via surface mail unless funds are sent to cover airmail postage. Foreign currency or checks will not be accepted. All orders must be in English

\section{TO ORDER, USE FORM ON REVERSE SIDE \\ Follow Instructions Bulow}

1. Please use separate forms for ordering publications and subscriptions.

2. Type or print your complete name and address, your order number (if any), your Deposit Account number (if applicable), Visa or Master Charge number (if applicable). and date in proper places at the top of the form. If order is to be shipped to a third party, flll in address at bottom of form. Wc request you order a minimum of $\$ 1.00$ worth of materials. Please include office/home telephone no.

3. When ordering publications, type or print the stock number, unit of issue (see front), quantity, title, price, and total payment enclosed.

4. When ordering subscriptions, or single issues of subscriptions, type or print the stock number, quantity. title, price; List ID (when available), and total payment enclosed. Allow 2-6 weeks plus mailing time for processing. Alt subscriptions are for one year, unless otherwise noted. Subscribers will be notified in ample time to renew.

5. Mail Original of Form to Superintendent of Documents, U.S. Government Printing Office, Washington, D.C. 20402. Retain the copy for your records.

6. If shipment is incorrect, return the shipping documents for adjustment. DO NOT RETURN BOOKS UNTIL NOTIFIED TO DO SO. All claims must be submitted within six months.

7. Call 202-783-3238 for publication information or for placing your Deposit Account, Visa or Master Charge order.

\section{THE FOILOWING INFORMATION EXPLAINS HOW YOUR REQUEST WAS PROCESSED}

Circle . Indicates the quantity of publications enclosed or scheduled for mailing.

B . : . The publication is not available at this time but we have scheduled mailing for a later date. Charges have been included on your order.

c. . We are unable to identify the publication from the information furnished.

1 . . This publication is not currently in stock. Please Inquire as to the availability and price in 60 days. You may inquire and order by telephone (202-783-3238) using your VISA. MASTER CHARGE. ar Suporintendent of Documents Deposit Account. This will expodite shipment of your publications.

L . . : The publication is not currently sold by this Office. We suggest you contact your local library which can refer you to a Federal Depository Library in your area for further assistance.

6 . . Publication has been replaced by another with similar but more resent informotion nin! it will be included in your shipment.

T . Publication has been replaced by another with similar but more recent information. Because of a difference in price. we are noting new ordering information on your request:

2 . . Requested or pertinent Information has been supplied. See attached. 


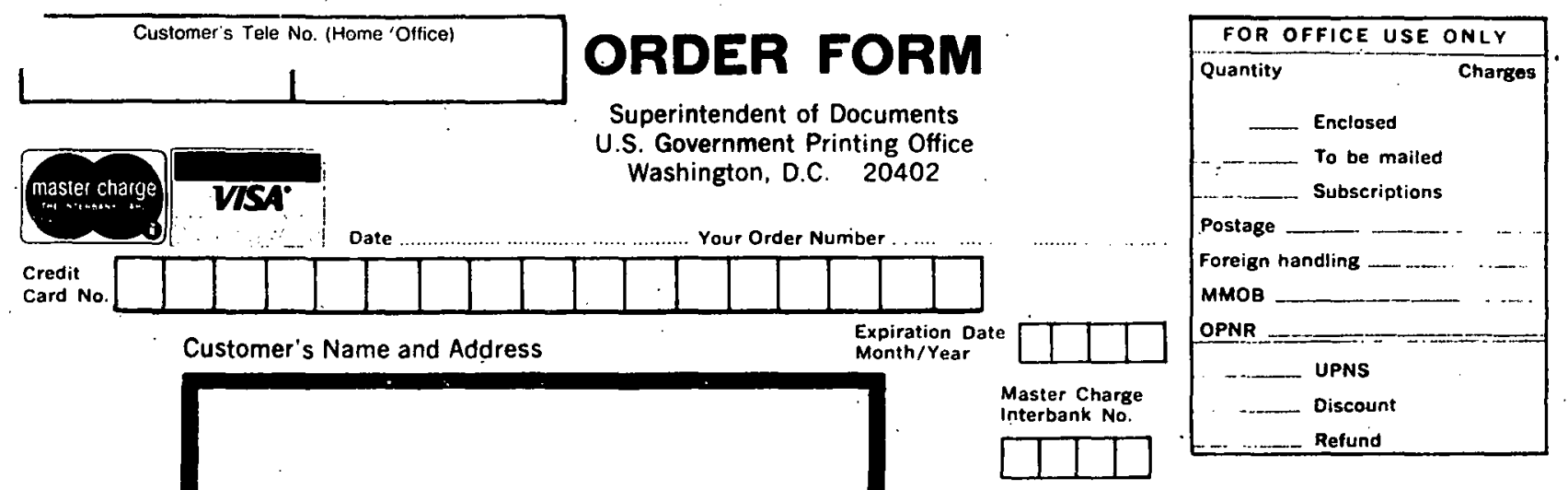

Superintendent of Documents

S. Government Printing Office

Deposit Account Number

ZIP

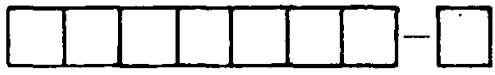

BEFORE USING THIS FORM, READ IMPORTANT INFORMATION ON REVERSE SIDE PLEASE PRINT OR TYPE ALL INFORMATION ON THIS FORM.

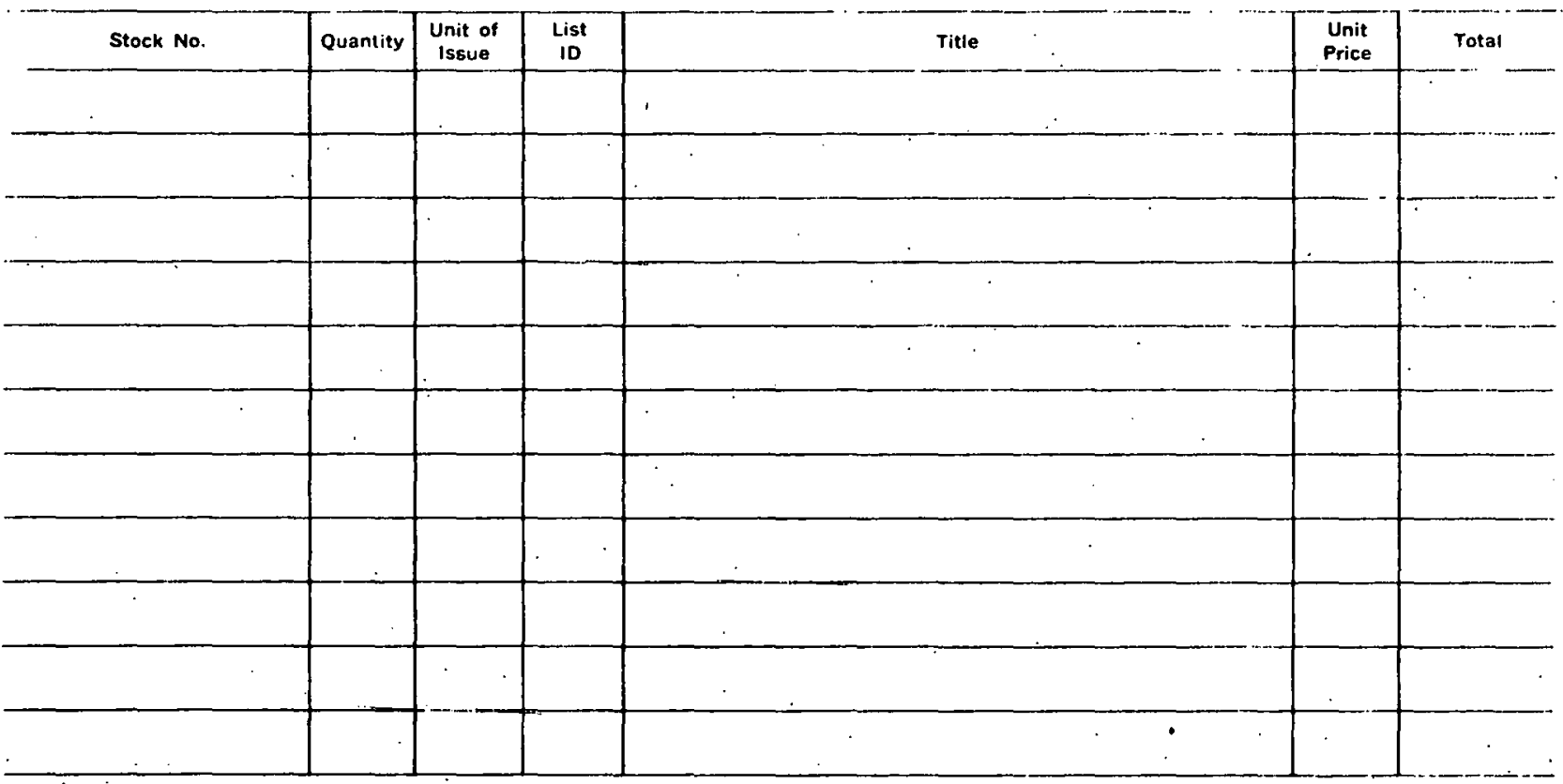

Expianation

Package containing multiple coples

Each-single copy

Pad containing multiple sheets

Set of multiple items

Kit of multiple items in a special container 


\section{INFORMATION CONCERNING YOUR ORDER OR INQUIRY}

Thank you for your interest in U.S. Government publications. If you have made a written inquiry. it is returned herewith for your reference. We have indicated the prices of publications currently available, or have provided the information requested to the best of our ability. Unless otherwise noted, prices are for single copies.

Regulations require payment in advance of shipment of publications. Please make checks or money orders payable to.the Superintendent of Documents. You may charge your order using your Deposit Account, Master Charge, or Visa. Do not send currency (bills or coins). or postage stamps.

Shipping is by non-priority mail or United Parcel Service. First class mailing is available at a higher rate. Contact us for rates if you desire this service. (202-783-3238)

With the exception of specially priced publications and subscriptions, a discount of 25 percent is allowed on quantity purchases (100 or more copies of a single publication). The same discount applies to orders from a bookdealer (any quantity) when the publiçations are mailed to the dealer's normal place of business. No discount will be allowed when publications and subscriptions are mailed to a third party (unless in quantities of 100 or more per title).

NON U.S. CUSTOMERS: International mailing regulations require special handling for whlch wè charge an additional 25 percent of the total cost of your order. Reunittance is .required in advance of shipping by draft on a United States or Canadian bank, by UNESCO coupons, or by International Postal Money Order made payable to the Superintendent of Documents. These orders are mailed via surface mail unless funds are sent to cover airmail postage. Foreign currency or checks will not be accepted. All orrters must be in English.

\section{TO ORDER, USE FORM ON REVERSE SIDEE}

Foliow Instructions Below

1. Please use separate forms for ordering publications and subscriptions.

2. Type or print your complete name and address, your order number (if any), your Deposil Account number (if applicable), Visa or Master Charge number (if applicable), and date in proper places at the top of the form. If order is to be shipped to a third party, fill in address at bottom of form. We request you order a minimum of $\$ 1.00$ worth of materials. Please include office/home telephone no.

3. When ordering publications, type or print the stock number, unit of issue (see front), quantity, title, price, and total payment enclosed.

4. When ordering subscriptions, or single issues of subscriptions, type or print the stock number, quantity, title; price, List ID (when available), and total payment enclosed. Allow 2-6 weeks plus mailing time for processing. All subscriptions are for one year, unless othenwise noted. Subscribers will be notified in ample time to renew.

5. Mail Original of Form to Superintendent of Ducuments, U.S. Government Printing Office, Washington, D.C. 20402. Retain the copy for your records.

6. If shipment is iiluilezt, rezurn the shipping documents for adjustment. DO NOT RETURN BOOKS UNTIL NOTIFIED TO DO SO. All claims must be submitted within six months.

7. Call 202-783-3238 for publication information or for plaring your Dopeoit Accuunt, Visa or Master Charge order.

\section{THE FOLLUWING INFORMATION EXPLAINS HOW YOUR REOIIFST WAS PROOCB3ED}

Circle . Indicates the quantity of publications enclosed nr ṣchodulod for inialliıg.

B . . The publication is not available at this time but we have scheduled mailing for a later date. Charges have haen included on your order.

c. We are unable to identify the publication from the information furnished.

i. . This publication is not currently in stock. Please inquire as to the availability and price in 60 days. You may inquire and order by telephone (202-783-3238) using your V!SA. MASTFR FUARGS, or Ouveilitentient of Documents Debosit arcent. This will expedite shipment of your publications.

L . . The publication is not currently sold by this Office. We suggest you contact your local library which can refer you to a Federa! Depository Library in your area far furthor assistanice.

S $\cdot$. Publication has been replaced by another with similar but hingre pocant informailiun and it will be included in. youl slipment.

T : . Publication has been replaced by another with similar but more recent information. Because of a difference in prlce, we are noting new ordering information on your request.

2 . . Requested or pertinent information has been supplted. See attached. 


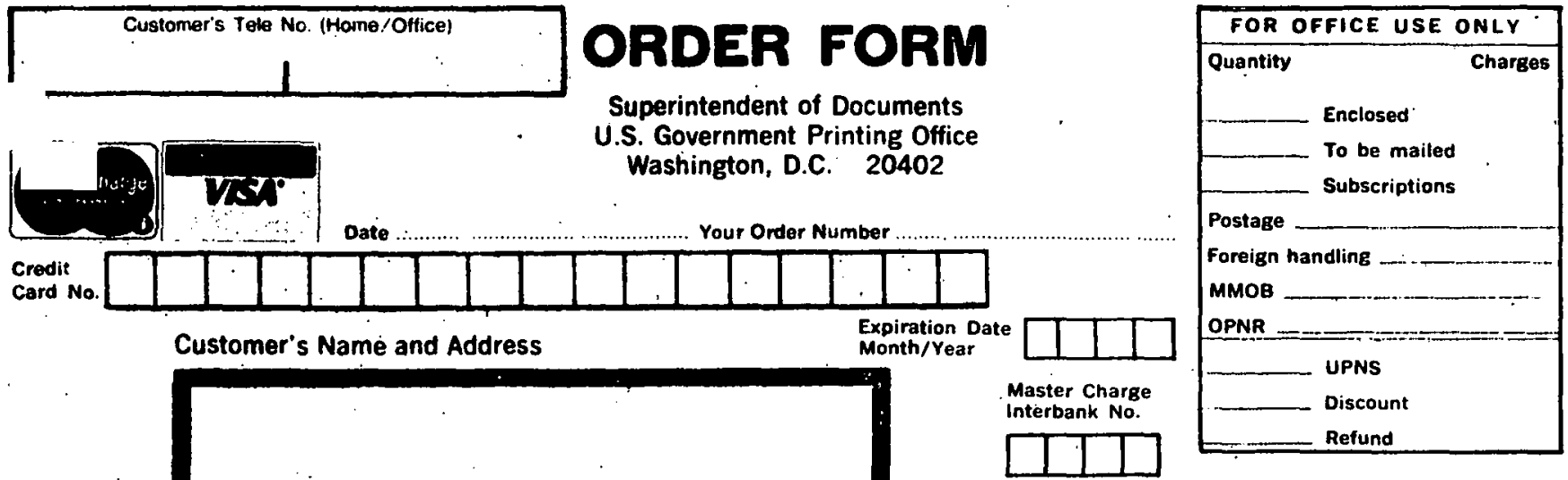

ZIP

Deposit Account Number

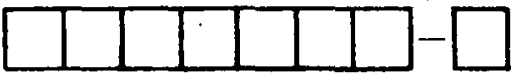

GEFORE USING THIS FORM, READ IMPORTANT INFORMATION ON REVERSE SIDE PLEASE PRINT OR TYFE ALL INIFORMATION ON THIS FORM.

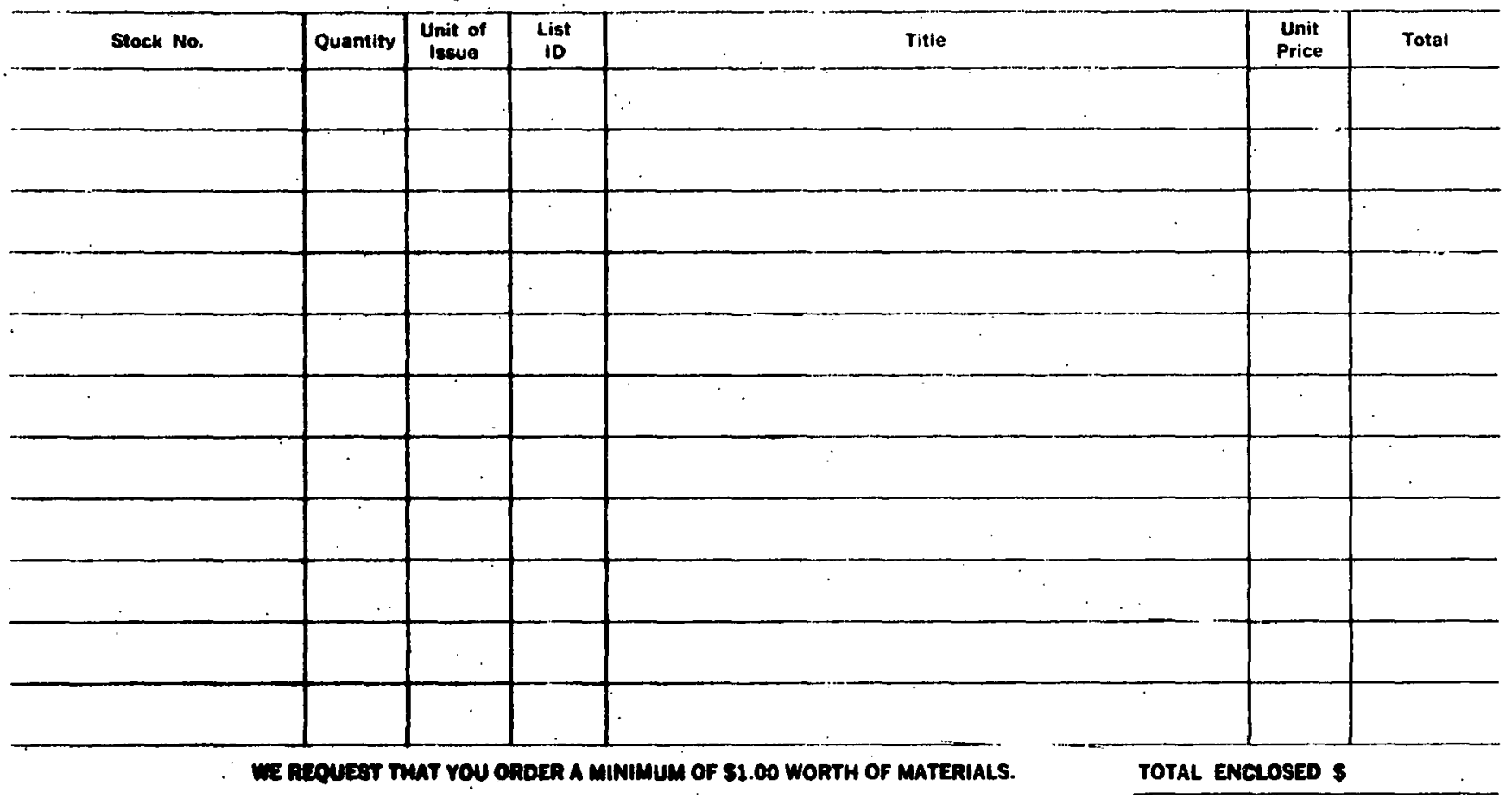

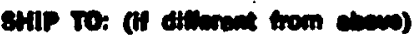

Unit of Issue

PK

EA

PO

SE

ENCLOSEO 


\section{INFORMATION CONCERNING YOUR ORDER OR INQUIRY}

Thank you for your interest in U.S. Government publications. !s you have made a written inquiry, it is retumed herewith for your reference. We have indicated the prices of publications currently available, or have provided the information requested to the best of our ability. Unless otherwise noted, prices are for single copies.

Regulations require payment in advance of shipment of publications. Please make checks or money orders payable to the Superintendent of Documents. You may charge your order using your Deposit Account, Master Charge, or Visa. Do not send currency (bills or coins) or postage stamps.

Shipping is by non-priority mail or United Parcel Service. First class mailing is available at a higher rate. Contact us for rates if you desire this service. (202-783-3238)

With the exception of specially priced publications and subscriptions, a discount of 25 percent is allowed on quantity purchases (100 or more copies of a single publication). The same discount applies to orders from a bookdealer (any quantity) when the publications are mailed to the dealer's normal place of business. No discount will be allowed when publications and subscriptions are mailed to a third party (unless in quantitios of 100 or more per title).

NON U.S. CUSTOMERS: International mailing regulations require special handling for which we charge an additional 25 percent of the total cost of your order. Remittance is required in advance of shipping by draft on a. United States or Canadian bank, by UNESCO coupons, or by International Postal Money Order made payable to the Superintendent of Documents. These orders are mailed via surface mail unless funds are sent to cover airmail postage. Foreign currency or checks will not be accepted. All orders must be in English.

\section{TO ORDER, USE FORM ON REVERSE SIUE \\ Follow Instructions Below}

1. Please usc separate forms for ordering publicatinns and aubseriptiuns.

2. Type or print your complete name and address, your order number (if any), your Deposit Account number (if applicable), Visa or Master Charge number (if applicable), and date in proper places at the top of the form. It order is to be shipped to a third party. fill in address at bottom of form. We request you order a minimum of $\$ 1.00$ worth of materials. Please include affice/home telephone no.

3. When ordering publleations, type or print the stock number, unit of issue (see front), quantity, title. price, and total payment enclosed.

4. When ordering subscriptions, or single issues of subscriptions, type or print the stock number, quantity. title, price, List ID (when evailable), and total payment enclosed. Allow 2-6 weeks plus mailing time for processing. All subscriptions are for one year, unless otherwise noted. Subscribers will be notified in ample time to renew.

5. Mail Original of Form to Superintendent of Documents. U.S. Government Printing. Office, Washington, D.C. 20402. Retain the copy for your records.

6. If shipment is incorrect. return the shipping documemts for adjustment. DO NOT RETURN BOOKS UNTIL NOTIFIEn Tn DO SO. All claims must be submitted within six months.

7. Call 202-783-3238 for publication information or for placing your Deposit Account, Visa or Master Charge order.

\section{THE FOLLOWING INFORMATION EXPLAINS HOW YOUR REQUEST WAS PROCESSED}

Circle , Indiratege tho quantity of publications ènclosed or scheduled for mailing.

a - . The publication is not available at this time but we have scheduled mailing for a later date. Charges have been included on your order.

C : - We are unable to identify the publication from the information furnished.

1 . - This publication is not currently in stock. Please inquire as to the availability and price in 60 days. You may inquire and order by telephone (202-783-3238) using your VISA, MASTER CHARGE, or Superintendent of Documents Deposit Account. This will expedite shipment of your publications.

L : : : The pubfication is not currently sold by this Office. We suggest you contact your local library which can refer you to a Bederal Depository Library in your area for further assistance.

8 ... Publication has been, replaced by another with similar but more recent information and it will be includer in your shipment.

$\boldsymbol{T}$. Publication has been replaced by another with similar but more recent information. Because of a differenco in price, we are noting new ordering information on your request.

2 . - Requested or pertinent information has been supplied. See attached. 
DOE Technical Information Center, P.O. Box 62, Oak Ridge, Tennessee 37830

*U.S. GPO: 1979-298-528

DOE Form IR'sado" (Previouis Edition

REPORT NO. are obsolete)
OFFICIAL REPORT REOUEST

REPORT DATE : CLASSIF. NO. OF

COPIES

WANTED

TITLE

AUTHORS

ORIGINATING ORGANIZATION

\section{\begin{tabular}{l|l|l}
$\because$ SOSTED FOR & SOURCE OF REFERENCE & CONTRACT NO. OF REPORT
\end{tabular}}

CERTIFICATION: REPORT IS TO BE USED FOR U.S. GOVERNMENT CONTRACT

\begin{tabular}{|l|l}
\hline CONTRACT NO. . & SIGNATURE \\
\hline
\end{tabular}

DATE OF REQUEST

(Enter vour complete mạiling address below.)

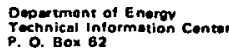

P. O. Box 82 Ridoo. Tonnowson 37830

OFFICIAL BUSINESS

POSTAGE ANO FEES PAIO DEPAATMENT OF ENERGY

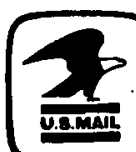

\section{IMPORTANT}

The Technical Information Center will supply fullsize copies of reports free of charge to official requesters if TIC has stock. The requesters should in all cases indicate below the action TIC should in all
take.

\section{ACTION REQUESTED}

(Check either No. 1 or No. 2 - not both.)

1. $\square$ Supply only if free, fuli-size stock is available.

2. D Supply if there is free, full-size stock but, if no stock is available, (Check (a) or (b) or (c).)

(a) $\square$ Send a quotation for a $\square$ blow-back and/or $\square$ microfiche copy from the reproduction contractor.

(b) $\square$ Have the reproduction contractor supply a blow-back copy of the report and

(i) $\square$ Bill requester or

(ii) $\square$ Charge to requester's deposit account.

(c) Have the reproduction contractor supply a microfiche and
(i) $\square$ Bill requester or

(ii) $\square$ Charge to requester's deposit account.

FOA TIC USE (Do not write in this space.)

1. Drawer No.

2. TIC has master copy ........ Yes

3. Report has been microfiched. . . Yes $\square$

4. Classification OU C S CATEGORY C.AUTHORIZED BY

$\square$ SEE REVERSE SIDE FOR REPLY 


\section{TIC RERLY' TO REPUHI REQUEST}

1. We are attemnting in obtoin copies. We will intorm you of nur results.

$\square$ 2. Aeport is not yet available. You will receive a copy when distribution is made.

D 3. The information in the report you requested has beell published in source notcd below.

4. Report cannot be identified from the information furnished. Please return this form with additional informatiun such as auther. title, issuing organization, contract numher, and sourcc of refeuence.

[5. Report is to be reprinted. Your request will be filled when copies are available.

$\square$ 6. Report is not yet available according to and we do not know when it will be issued.

7. We have been informed by the issuing organization that this report received internal distribution only.

$\square$ 3. Report is obsolete. $\square$ Superseded by report number

$\square$ 9. Report is illegible to degree that precludes reproduction.

$\square$ 10. Report is nor available in mirrofiche form.

$\square$ 11. Not an DOE publication. $\square$ Request from originatirig organization.

Request from source noted below. Request from

$$
\square \text { DDC } \square \text { NASA }
$$

\section{$\square$ NTIS}

12. Mav be purchased from soúrce noted below.

$\square$ 13. Other. Sec below. 
DOE Technical Information Center, P.O. Box 62, Oak Ridge, Tennessee 37830

DOE Form-IRSTO- (Previouis Editioñs (4-78) are obsolece)

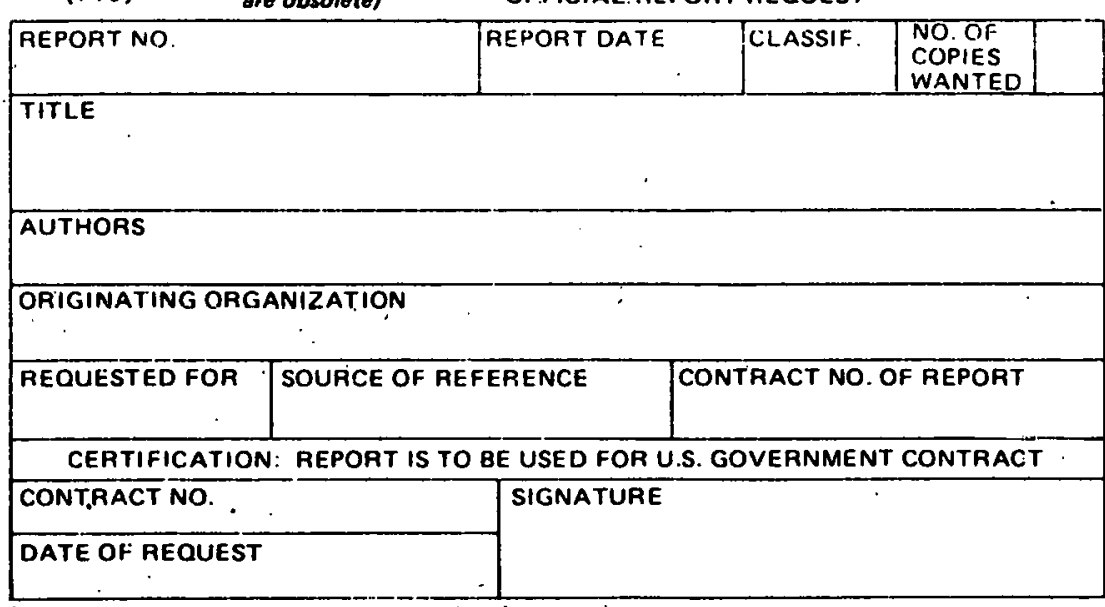

(Enter vour comolete mailing address below.)

From: Depertment of Enoroy Canter

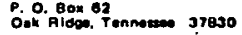
OPPICIAL BUEINGES OPPICIAL OUEINEES
PENALTY FOR PAIVATE USE.
$\mathbf{3}$
POSTAGE AND FEES PAID DEPARTMENT OF ENERGY

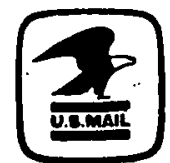

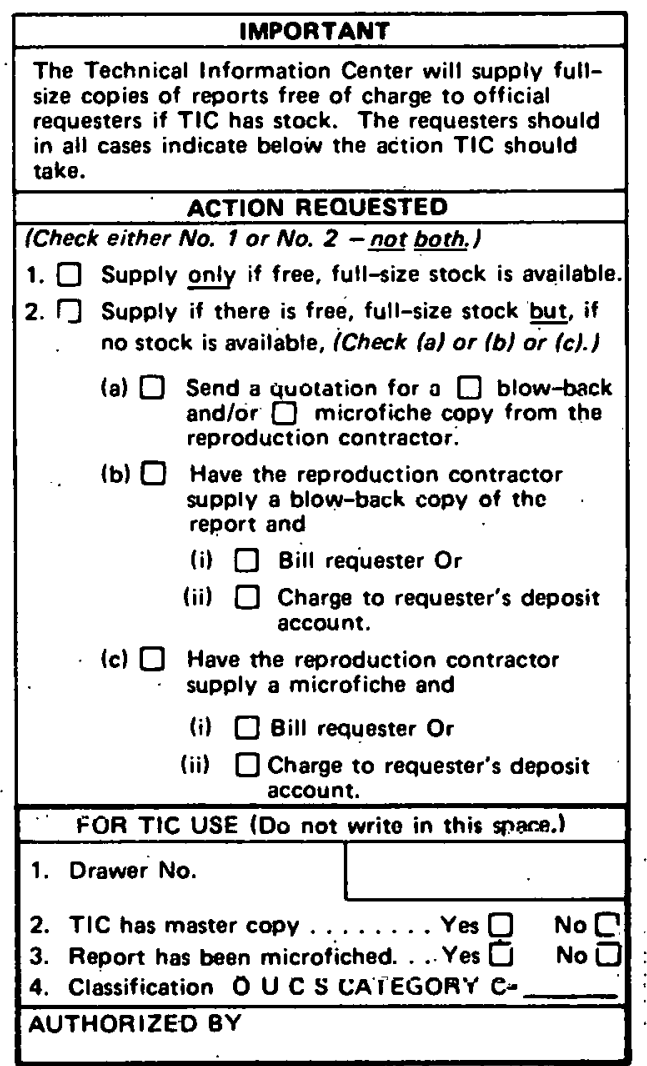

- SEE REVERSE BIDE FOA REPLY 
TIG REPLY,TO REPOAT HEUUEST

$\square$ 1. We are attempting to obtain copies. We will inform you of our results.

$\square$ 2. Report is not vet available. You will receive a copy. when distribution is made.

$\square$ 3. The information in the report you requested has beell published in source noted below.

$\square$ 4. Repurt cannot be identified from the information furnished. Please return this form with additional information such as author, title, issuing organization, contract number, and source of reference.

¿ 5. Rcport is to be reprinted. Your request will be filled when copies are available.

$\square$ 6. Report is not yet available according to and we do not know when it will be issued.

$\square$ 7. We have been informed by the issuing organization that this report received internal distribution only.

$\square$ 8. Report is obsolete. $\square$ superseded by report number

$\square$ 9. Report is illegible to degree that precludes reproduction.

$\square$ 10. Report is not available in microfiche form.

$\square$ 11. Not an DOE publication. $\square$ Request from originating organization. Request from . $\square$ DDC $\square$ NASA $\square$ NTIS

$\square$ 12. May be purchased from source noted below.

$\square$ 13. Other. See below. 
DOE Technical Information Center, P.O. Box 62, Oak Ridge, Tennessee 37830

COOE Form 1R-540 (Provioious Editionis
(4.78) are obsoletel

\begin{tabular}{|l|l|l|l|l|}
\hline REPORT NO. & REPORT DATE & CLASSIF. & $\begin{array}{l}\text { NO.OF } \\
\text { COPIES } \\
\text { WANTED }\end{array}$ \\
\hline TITLE & \\
\hline AUTHORS & & \\
\hline ORIGINATING ORGANIZATION & CONTRACT NO. OF REPORT \\
\hline REOUESTED FOR & SOURCE OF REFERENCE & \\
\hline CERTIFICATION: REPORT IS TO BE USED FOR U.S. GOVERNMENT CONTRACT \\
\hline CONTRACT NO. & SIGNATURE \\
\hline DATE OF REQUEST &
\end{tabular}

- (Enter vour comolete mailing address below.)

From: Depmetront of Enorgy

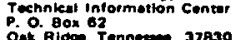

OFficial aUsiness

OFFICIAL QUSINEBS
POSTAGE ANO fEes PAIO DEPARTMENT OF ENERGY

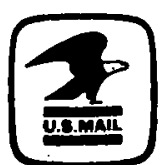

*U.S. GPO: $1979-298.528$ IMPORTANT

The Techinical Information Center will supply fullsize copies of reports free of charge to official

requesters if TIC has stock. The requesters should in all cases indicate below the action TIC should take.

\section{ACTION REQUESTED}

(Check either No. 1.or No. 2 - nof both.)

1. $\square$ Supply only if free, full-size stock is available.

2. D Supply if there is free, full-size stock but, if no stock is available, (Check (a) or (b) or (c).)

(a) $\square$ Send a quotation for a $\square$ blow-back and/or $\square$ microfiche copy from the reproduction contractor.

(b) $\square$ Have the reproduction contractor supply a blow-back copy of the report and
(i) $\square$ Bill requester Or
(ii) $\square$ Charge to requester's deposit
(ii) $\square$ account.

(c) $\square$ Have the reproduction contractor supply a microfiche and

(i) $\square$ Bill requester Or

:(ii) $\square$ Charge to requester's deposit account.

FOR TIC USE (Do not write in this space.)

1. Drawer No.

2. TIC has master copy ........ Yes $\square$ No $\square$

3. Report has been microfiched. . . Yes $\square$ No $\square$

4: Classifiootion 011 r. S C.ATEGORY C-

AUTHORIZED BY

SEE REVERSE SIDE FOA REPLY 


\section{TIC REPLY TO REPORT RFOIIFST}

1. We are attempting to obtain copies. We will inform you of our resu!!ts.

2. Report is nol yet avallable. You will receive a copy when distribution is made.

] 3. The information. in the report you requested has been published in source noted below.

4. Report cannot be identified from the information furnished. Please return this form with additional information such as author. title, issuing organization, contract number, and source of refercnce.

5. Report is to be reprinted. Your request will be filled when copies are available.

6. Report is not yet available according to and we do not know when it will be issued.

$\square$ 7. We have been informed by the issuing organization that this report received internal distribution only.

8. Report is obsolete. $\square$ Superseded by report number

9. Report is illegible to degree that precludes reproduction.

$\square$ 10. Report is not available in microfiche form.

$\square$ 11. Not an DOE publication. Reyuest trom

$\square$ Request from originating organization. $\bigcap$ Request from source notod beloov.

$\square$ 12. Mav be purchased from source noted below.

$\square$ 13. Other. See below. 

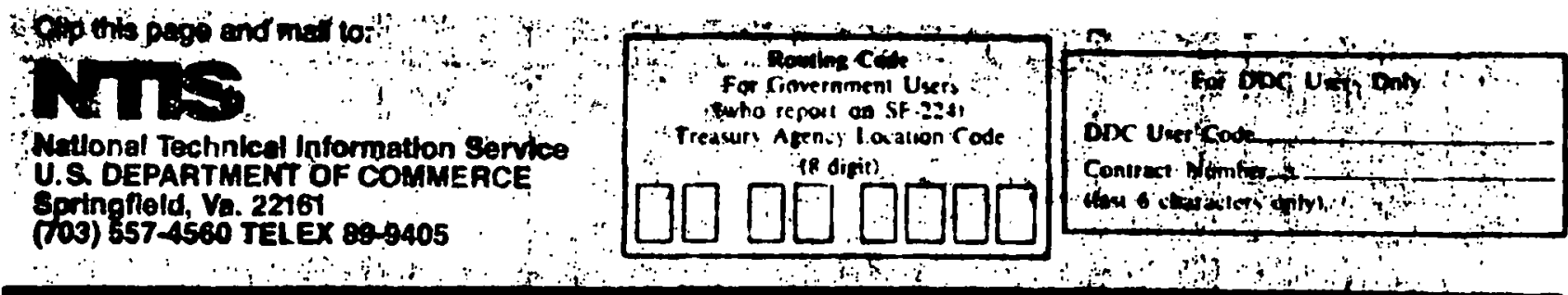

see roveree for how to order.

PURChaser's Name

\section{Address}

Cily, State, 7.JP

Phone

\section{METHOD OF. PAYMENT}

C Charge miy NTIS Deposit Account nu.

Check enclosed for $\$$

$\square$ Purchase urder attached"

$\square$ Bill me* (not available out side the North American continent.)

-Add $\$ 5.00$ per urder for deferred payment and sign below.

$\square$ Charge to my Anierican Express Card account number.

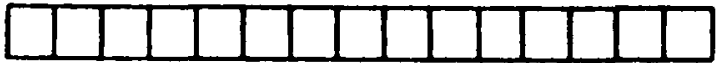

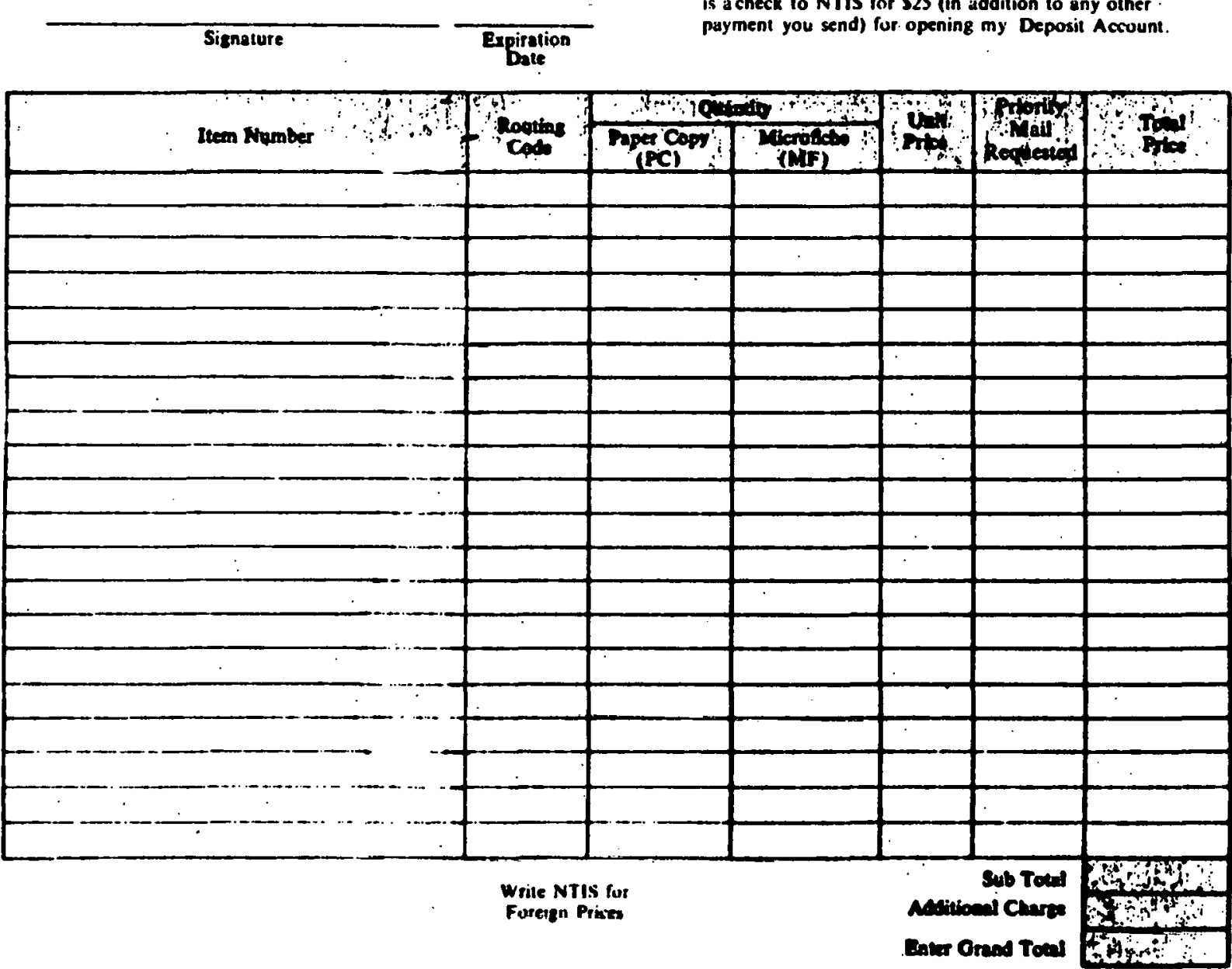




\section{HOW TO OADER}

Please completë the Order section (on reverse side) in tull to place your order. Fill out Ship to section only if you wish your order shipped to another address.

For additional information on NTIS products and services, fill out the Information Requested section (below) in full including mailing address.

You can opt for priority mail for \$3 North American continent.; $\$ 4$ outside North America charge per item. Just check the Priority Mail box. If you're really pressed for time, call the NTIS Rush Handling Service (703) 557-4700.

For a $\$ 10$ charge per item, your order will be sent priority mail within 24 hours. Or, for a 56 per item charge, you can pick up your order in the Washington Information Center \& Bookstore or at our Springfield Operations Center within 24 hours.

You may also place your order by telephone or TELEX if you have an NTIS Deposit Account or an American Express card. The order desk number is (703) $557-4650$ and the TELEX number is $89-9405$.

NTIS can label each document for routing within your organization. If you want this service put your routing code up to 8 characters in the box marked Routing Code

Thank you for your interest in NTIS. We appreciate your order.

\section{INFORMATION REQUESTED}

\section{SEND ME:}

An application for an NTIS Deposil Account NTIS-PR-33-4U3

Information Services General Catalog. NTIS-PR-154-403

Name

Address

City, State, ZIP

Telephone
Environmental Subscriptions Brochure. NTIS-PR-373-403 Energy Cotalng. NTIS PR-378-103

Abstract Newsletters Brochure. NTIS-PR-205-403

Published Search Catalog. NTIS-PR-186-403

D An epplication for Special Credit Accounts for state/local Bovernments and state universities. NTIS-PR-220-403

D Domestic Price Cude list. NTIS-PR-360-3-403

D Foreign Price Code LisI. NTIS-PR-360-4-403

Oiher

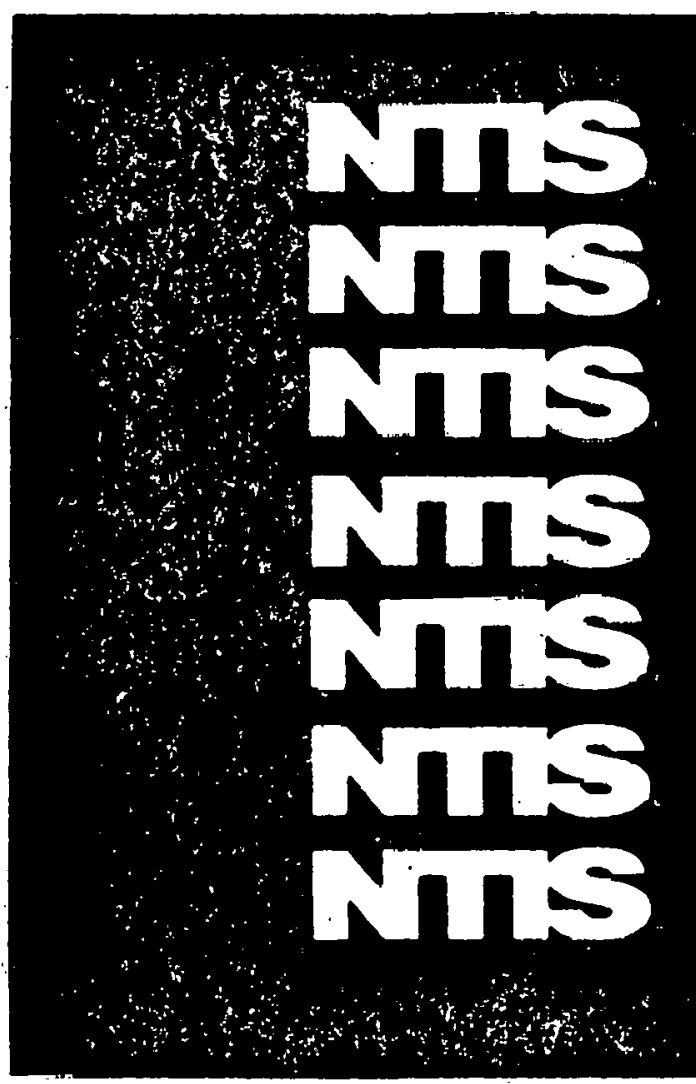

Proaso Place Stamp Nom
Notional Tochnical information sorvice U.S. DEPAATMENT OF COMMERCE Springfield. VA 22161 


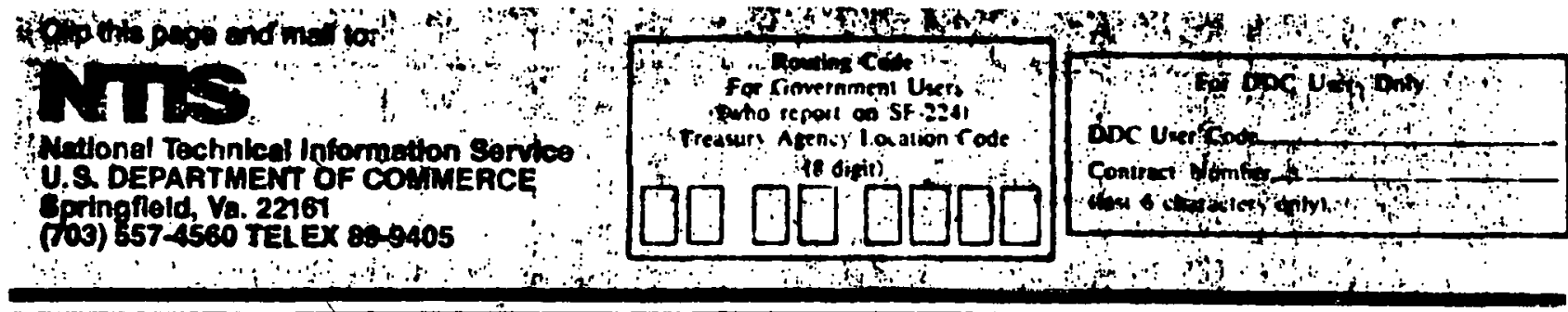

8ee reveres for how to ender.

PURCHASER'S Name

Address

Cily, Stute, 7JP

\section{METHOD OF PAYMENT}

$\square$ Charge my NTIS Deposit Account nu.

$\square$ Check enclosed for 5

$\square$ Purchase urder attached

$\square$ Bill me" (not available outside the North American continent.) -Add $\$ 5.00$ per urder for deferred payment and sign below.

$\square$ Charge $10 \mathrm{~m}$ ) Anierican Express Card account number.

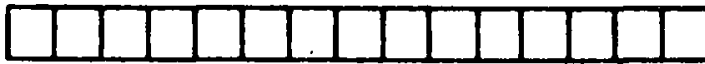

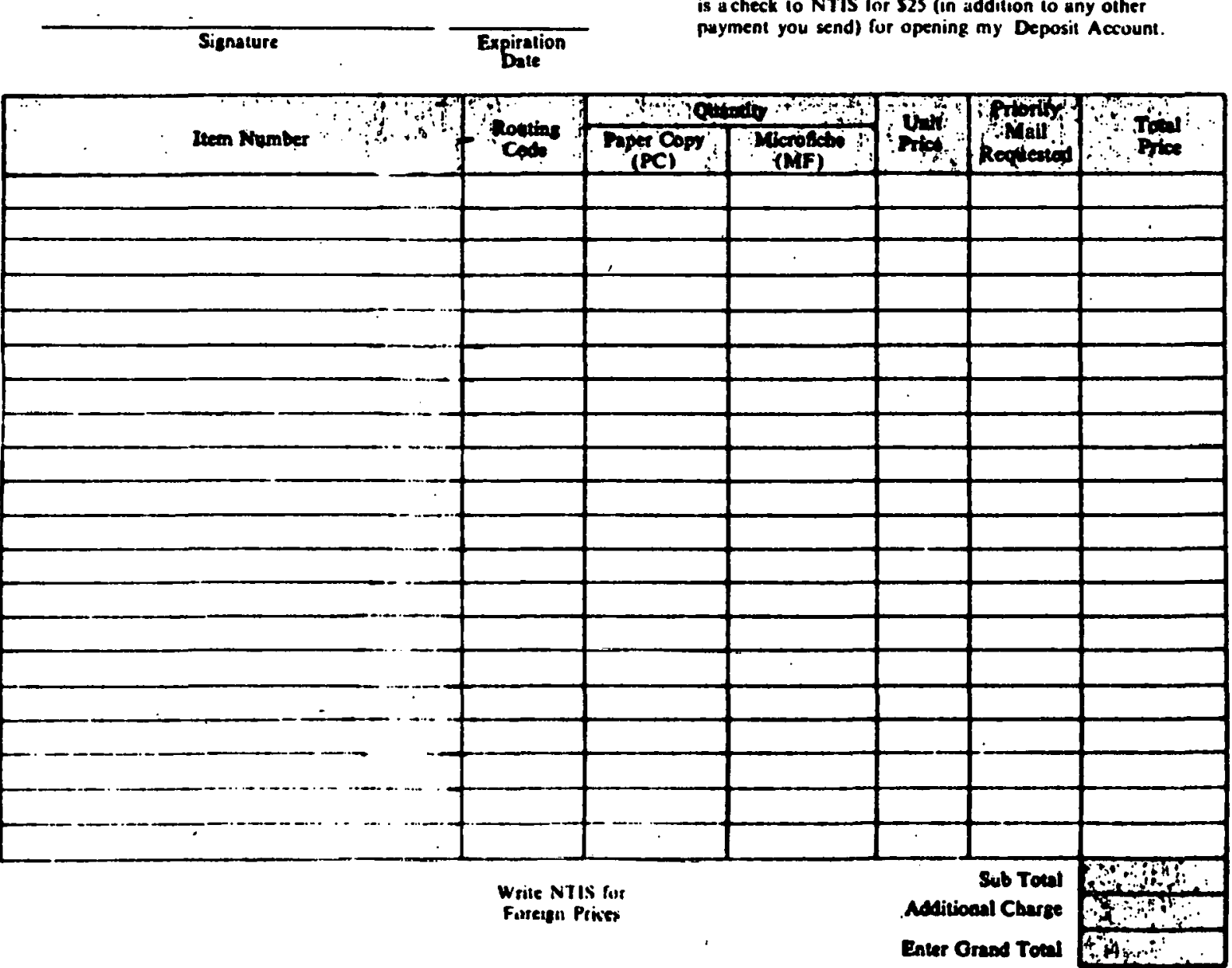

SMIP TO:

(Enter if different from address above)

Name

Organization

Address

City, State, ZIP

Dopen a DEPOSIT ACCOUNT in above nume. Enclused is a check 10 NTIS for $\$ 25$ (in addition 10 any other puyment you send) fur opening my Deposit Account. 


\section{MOW TO OAOER}

Please complete the Order section (on reverse side) in full to place your order. Fill out Ship to section only if you wish your order shipped to another address.

For additional information on NTIS products and services, fill out the Information Requested section (below) in full including mailing address.

You can opt for priority mail for \$3 North American continent.; $\$ 4$ oulside North America charge per item. Just check the Priority Mail box. If you're really pressed for time, call the NTIS Rush Handling Service (703) 557-4700.

For a $\$ 10$ charge per itern, your order will be sent priority mail within 24 hours. Or, for a $\$ 6$ per item charge, you can pick up your order in the Washington Intormation Center 8 Bookstore or at out Springfield Operations Center within 24 hours.

You may also place your order by telephone or TELEX if you have an NTIS Deposit Account of an American Express card. The order desk number is (703) 557.4650 and the TELEX number is 89-9405

NTIS can label each document for routing within your organization. If you want this service put your routing code up 108 characters in the box marked Rouling Code.

Thank you for your interest in NTIS. We appreciate yout order.

\section{INFORMATION REQUESTED}

\section{SEND ME:}

$\square$ An application for an NTIS Depusil Accuuni. NTIS-PR-33-403

Information Services General Catalog. NTIS-PR-IS4-403

Name

Address

City, State, ZIP.

Telephone
Environmental Subscriptions Brochure. NTIS-PR-373-403

Eica By Cualug. NTIS PR-37to-40,

Abstract Newsletters Brochure. NTIS.PR-205-403

Dublished Search Catalog. NTIS-PR-186-103

D. An applicution for Special Credit Accounts fór state/locat zovemments and state undversities. NTIS-PR-220-40.3

D Domestic Price Code list. NTIS-PR-360-3.403

D Foreign Price Code LisI. NTIS-PR-360-4-403

Oiher

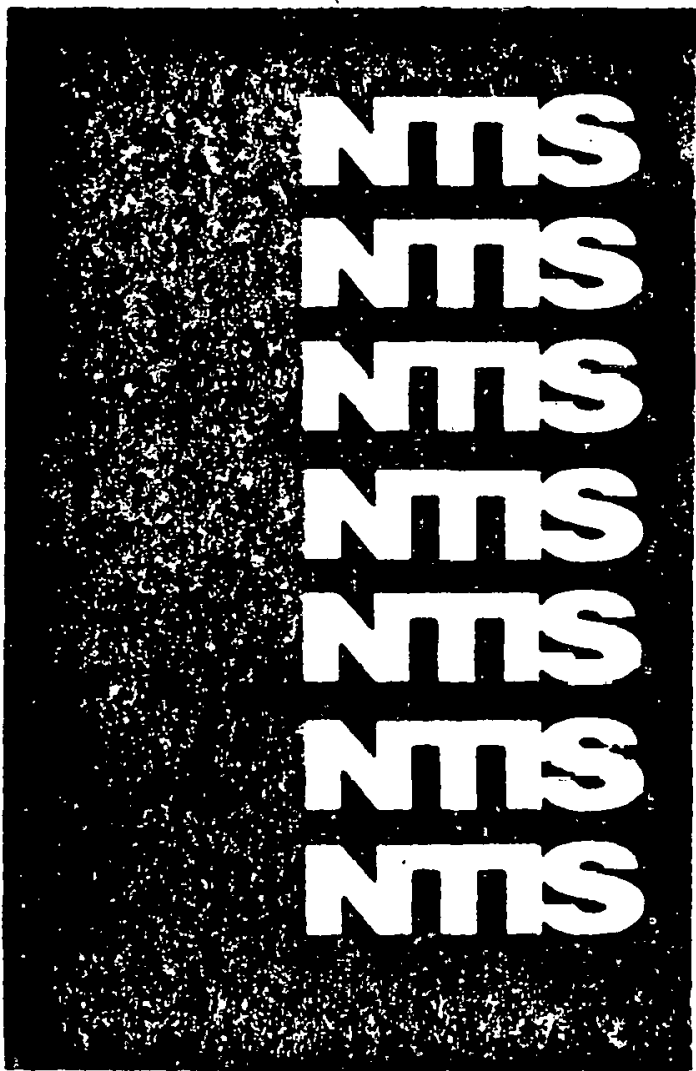

National Technical Information sorvice U.S. DEPARTMENT OF COMMERCE Springfleld, VA 22161 

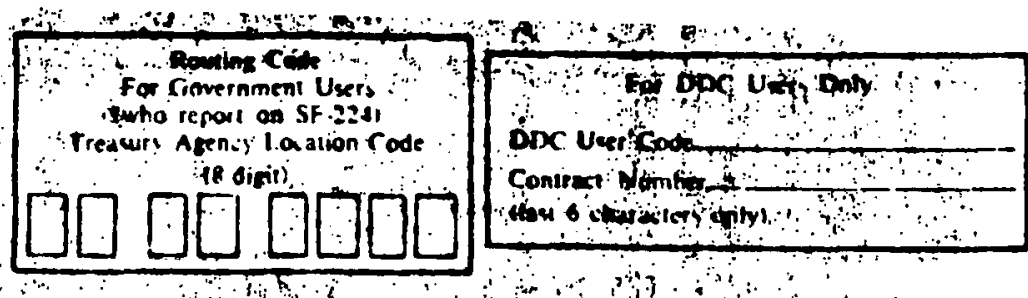

see reveree for how to order.

PLRChaser'S Name

Address

City. Slate. ZIP

Phone

\section{METHOD OF PAYMENT}

Charge my NTIS Deposit Account nu.

Check enclosed for $\$$

$\square$ Purchase urder allached•

$\square$ Hill me* (not available outside the North American continent.) -Ada $\$ 5.00$ per vider for deferred payment and sign below.

$\square$ Charge to $\mathrm{m}$ ) Anierican Express Card account number.
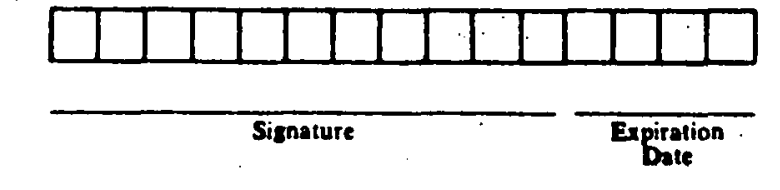

Swir tro:

(Enter if different from address above)

Name

Organization

Address

City. State, ZIP

DOpen a DEPOSIT ACCOUNT in above name. Enclosed is a check 10 NTIS for S2S (in addition to any other payment you send) for opening my Deposit Account.

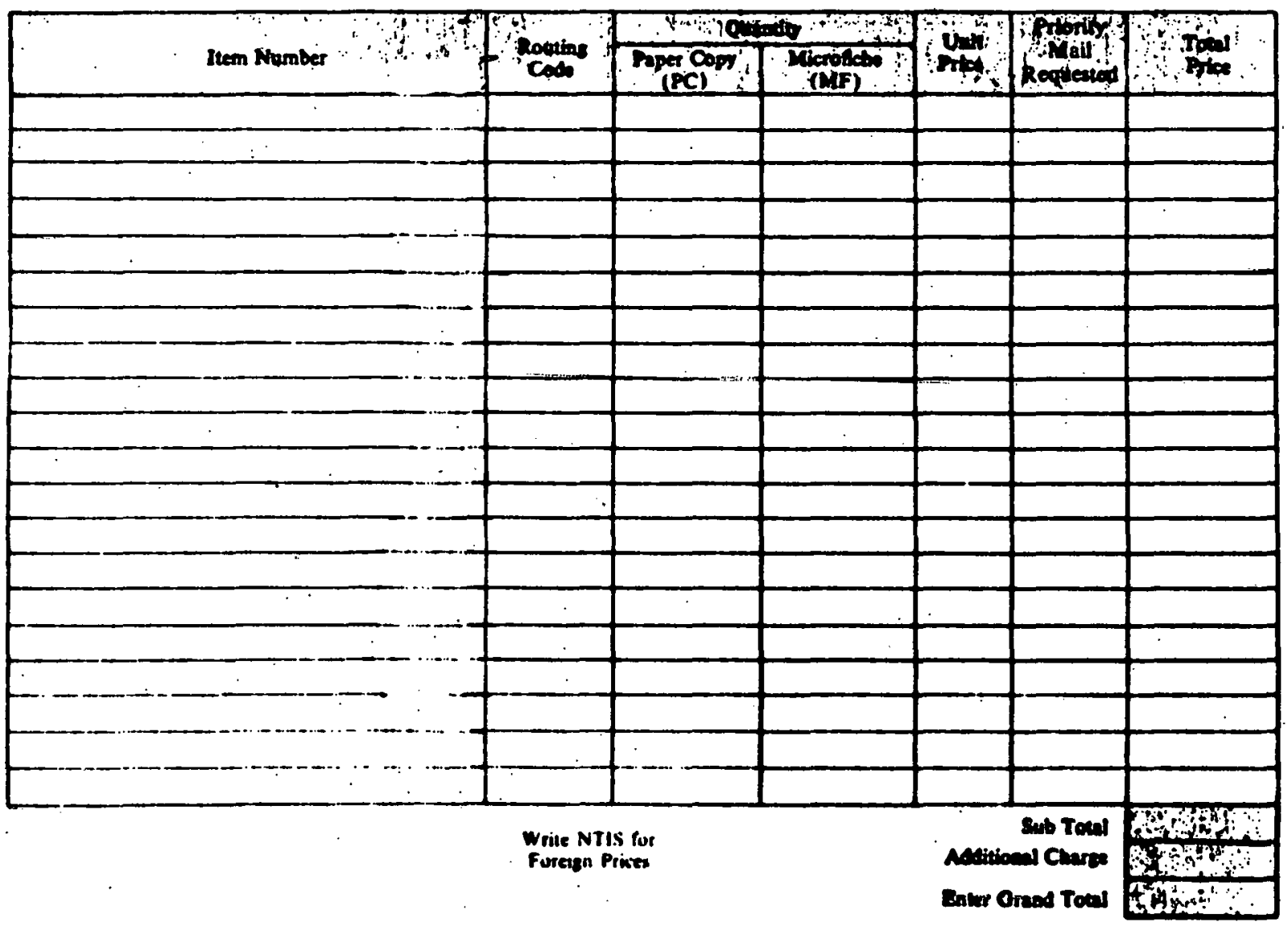




\section{MOW TO ORDER}

Please complete the Order section (on reverse side) in full to place your order. Fill oul Ship to section only if you wish your order shipped to another address.

For additional information on NTIS products and services, fill out the Information Requested section (below) in full including mailing address.

You can opt for priority mail for $\$ 3$ North American continent.; $\$ 4$ outside North America charge per item. Just check the Priority Mail box. If you're really pressed for time, call the NTIS Rush Handling Service (703) $557-4700$

For a $\$ 10$ charge per item, your order will be sent priority mail within 24 hours. Or, for a $\$ 6$ per itern charge, you can pick up your order in the Washington Information Center \& Bookstore or at our Springfield Operations Center within 24 hours.

You may also place your order by telephone or TELEX if you have an NTIS Deposit Account or an American Express card. The order desk number is (703) $557-4650$ and the TELEX number is 89-9405.

NTIS can label each document for routing within your organization. If you want this service put your routing code up to 8 characters in the box marked Routing Code

Thank you for your interest in NTIS. We appreciate your order.

\section{INFORMATION REQUESTED}

\section{SEND ME:}

An application for an NTIS Deprosi Account.

NTTis PR 33- 103

Information Services General Catalog. NTIS-PR-154 -403

Name

Address

City, State, ZIP

Telephone

$\square$ Environmental Subscriptions Brochure. NTIS-PR-373-403
Energy Catalog. NTIS PR-378-403
$\square$ Abstract Newsletters Brochure. NTIS-PR-205-403
$\square$ Published Search Catalog. NTIS-PR-186-403
An application for Special Credit Accounts for state/local
governments and state universities. NTIS-PR-220-403
Domestic Price Code list. NTIS-PR-360-3-403
Foreign Price Code List. NTIS-PR-360-4-403
$\square$ Other

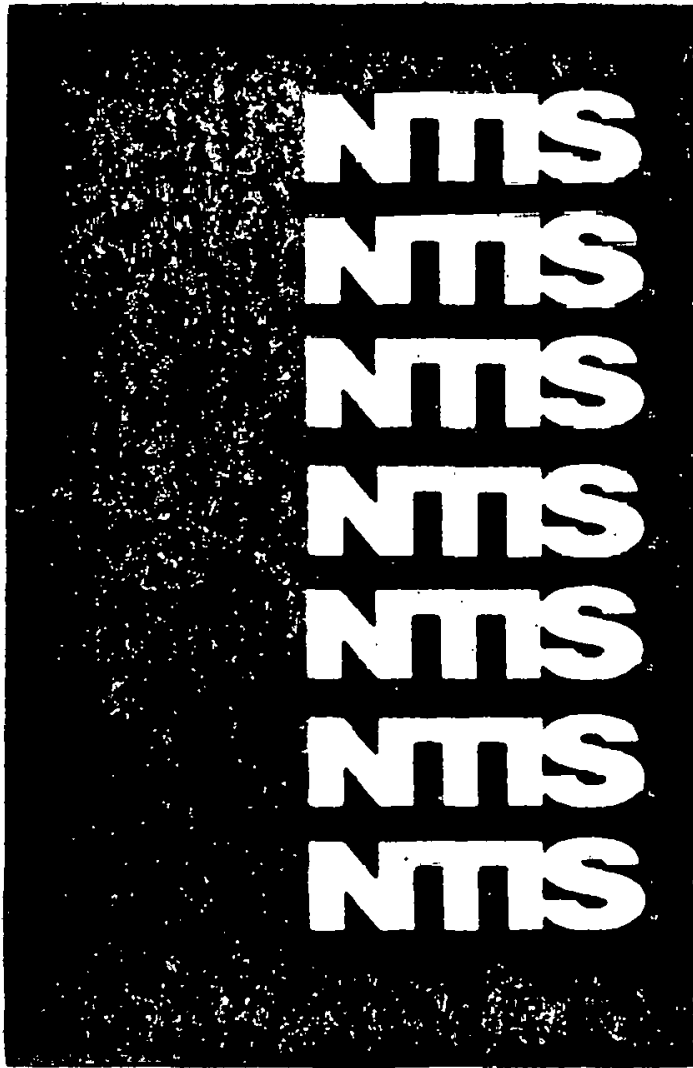

National Technlcal Information Service U.S. DEPARTMENT OF COMMERCE Springfield, VA 22161 


\section{United States}

Department of Energy

Washington, D.C. 20585
THIRD -CLAASSMAII

POSTAGE E FEES PAID

U.S. DEPT. OF FNFHGY

PERMITNO.G 20

Official Business

Penalty for Private Use, $\$ 300$ 